DEPARTMENT OF"THE INTERIOR

FrankLIN K. LANE, Secretary

United States Geological Survey GeORGe OTIS SMITH, Director

Water-Supply Paper 427

BIBLIOGRAPHY AND INDEX •

OF THE

PUBLICATIONS OF THE UNITED STATES

GEOLOGICAL SURVEY RELATING

TO GROUND WATER

BY

OSCAR E. MEINZER

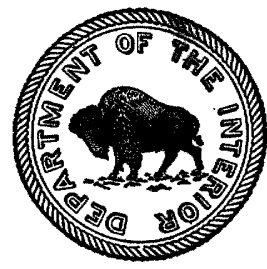

WASHINGTON

GOVERNMENT PRINTING OFFIOE

1918 


\section{CONTENTS.}

Introduction: Page.

Work done by the.United States Geological Survey. $. . . \ldots \ldots \ldots \ldots \ldots . .1$

Scope of the bibliography and index............................ 2

How to obtain the papers listed in the bibliography $\ldots \ldots \ldots \ldots \ldots \ldots . .3$

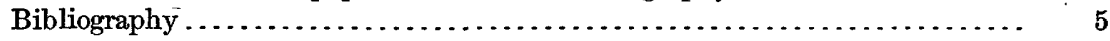

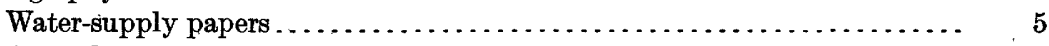

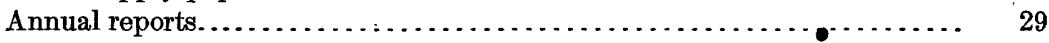

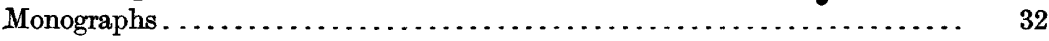

Professional papers............................................. 33

Bulletins. . . . . . . . . . .

Reports on mineral resources............................. 41

Geologic folios................................................. 42

Cooperative reports not published by the United States Geological Survey. $\quad 45$

Miscellaneous spring records................................ 47

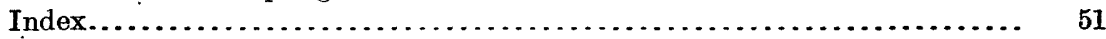

\section{ILLUSTRATION.}

Prate I. Map of the United States showing areas covered by the principal reports of the United States Geological Survey relating to ground water........................................ In pocket. 


\title{
BIBLIOGRAPHY AND INDEX OF THE PUBLICATIONS OF THE UNITED STATES GEOLOGICAL SURVEY RELATING TO GROUND WATER.
}

\author{
By Oscar E: MeInzer.
}

\section{INTRODUCTION.}

\section{WORK DONE BY THE UNITED STATES GEOLOGICAL SURVEY.}

The work of the United States Geological Survey includes investigations of the natural waters of the United States, both those which occur above and those which occur below the land surface. The waters below the surface are known as subsurface, subterranean, underground, or ground waters. According to the present usage of the Geological Survey the waters that occur below the surface in zones of saturation are called ground waters, and it is to these waters that the present bibliography and index applies.

In 1885 the Geological Survey published a paper by T. C. Chamberlin entitled "The requisite and qualifying conditions of artesian wells." But aside from this well-known paper it published practically nothing on the subject of ground water in the first 10 years of its existence except "Lists and analyses of mineral springs," by A. C. Peale; annual statistics on the production of mineral water, by A. C. Peale; and papers on the waters of Yellowstone National Park, by W. H. Weed and by F. A. Gooch and J. E. Whitfield.

In 1888 , by act of Congress, the Geological Survey undertook irrigation investigations in the arid regions of the United States, which soon led to the problem of irrigation with artesian water. The Eleventh Annual Report, published in 1891, contains a section on artesian irrigation on the Great Plains and records of wells in 7 western States. In the Thirteenth Annual Report, published in 1893, there is a discussion by F. H. Newell on the occurrence and quantity of ground water in arid regions, with statistics on artesian wells and irrigation with well water in the United States. The need for more detailed information on the ground waters of the country became evident and resulted in a number of investigations. The first report dealing exclusively with eastern conditions was a paper by W J McGee, entitled "Potable waters of eastern United States," published in 1893. Systematic and extensive survey of the ground- 
water resources of the United States was begun in 1903, when the division of hydrology was organized, with N. H. Darton in charge of the work in the West and M. L. Fuller in charge of the work in the East. Since that time the ground-water survey has been carried steadily forward, and the methods of investigation have gradually been refined and standardized. From 1908 to 1912 the division was in charge of W. C. Mendenhall, and since 1912 it has been in charge of O. E. Meinzer.

Altogether a vast amount of accurate information of both scientifio and economic value has been accumulated on the ground waters of the United States. A total of 609 papers have been published (in 454 volumes) which contain information on the subject of ground water, of which 307 papers (in 171 volumes) relate primarily to this subject. Many of these papers include detailed maps showing the ground-water conditions in specific areas. In addition to the published reports there is a great quantity of data as yet unpublished on file at the Geological Survey.

Although the aggregate of the work done on the ground-water resources of the United States is great and includes some work in every State, yet the country is so large that extensive regions are still very inadequately covered, and the amount of work that remains to be done is indefinitely great.

\section{SCOPE OF THE BIBLIOGRAPHY AND INDEX.}

The present bibliography includes all publications prepared in whole or in part by the Geological Survey that treat any phase of the subject of ground water or any subject directly applicable to ground water, such as windmills, pumps, and other lifting devices, methods of water analysis, and methods of measuring the flow of water. It has a more limited scope than the bibliography by M. L. Fuller on ground-water papers published by the Geological Survey, 18791904 (Water-Supply Paper 120), in that it does not include papers that touch on the subject only incidentally. As in Fuller's bibliography, there is a brief abstract of each paper that is listed with special reference to its content on ground water. For papers dealing chiefly with other subjects page references are given to the parts relating to ground water.

The 454 volumes listed in this bibliography, comprise 207 watersupply papers, 22 annual reports, 4 monographs, 11 professional papers, 47 bulletins, 33 reports on mineral resources, 118 geologic folios, and 12 reports not published by the United States Geological Survey. Of the 171 volumes relating primarily to ground water 123 are water-supply papers.

The index includes only subjects relating to ground water. The areas covered by the reports are given under the individual States. 
For more definite information on reports covering specifio localities the index map ( $\mathrm{Pl}$. I, in pocket) should be consulted. The map does not, however, show all reports listed in the bibliography but only such as cover specific areas more or less thoroughly. Under the individual States in the index are given references to many reports which it was not practicable to show on the map.

\section{HOW TO OBTAIN THE PAPERS IISTED IN THE BIBLIOGRAPHY.}

The publications of the United States Geological Survey listed in this bibliography, except the monographs and geologic folios, can be obtained free as long as the Survey's stock lasts. An asterisk (*) indicates that this stock has been exhausted. Many of the publications so marked may, however, be purchased at the prices indicated from the Superintendent of Documents, Washington, D. C. If there is an asterisk and no price is given the publication can no longer be obtained from the Government.

Monographs can be purchased at the prices indicated from the Superintendent of Documents, Washington, D. C.

Geologic folios can be purchased at the prices indicated, from the Director, United States Geological Survey, Washington, D. C., excépt those marked with an asterisk $\left(^{*}\right)$, which are out of stock and can no longer be obtained from the Government. Those priced at 5 cents have been more or less damaged by smoke or water.

The publications of the Geological Survey can be consulted in most of the principal libraries of the country and also at the branch offices of the Geological Survey in a number of the larger cities.

The cooperative reports listed on pages $45-47$ are not published by the United States Geological Survey and are not distributed by the Federal Government. 



\section{BIBLIOGRAPHY.}

An asterisk (*) indicates that the publication referred to is out of stock (see section headed "How to obtain the papers listed in the bibliography," p. 3).

\section{WATER-SUPPLY PAPERS.}

*1. Pumping water for irrigation, by H. M. Wilson. 1896.57 pp., 9 pls.

Describes various pumps and curious and antiquated lifting devices used chiefty in India and Egypt; also engines, windmills, water wheels, and other devices for producing power for lifting water; also storage reservoirs for holding pumped water until needed for irrigation.

*2. Irrigation near Phoenix, Ariz., by A. P. Davis. 1897. 98 pp., 31 pls. $15 \mathrm{c}$.

Describes chiefly irrigation with surface waters but also contains some well data and discusses briefly the quantity of underflow (pp. 86-92). For more comprehensive reports on the region see Water-Supply Papers 136 and 375-B.

*4. A reconnaissance in southeastern Washington, by I. C. Russell. 1897. 96 pp., 7 pls. $15 \mathrm{c}$.

Describes the geography and geology, gives some well data, and discusses the artesian conditions and the irrigation prospects of an indefinite region in southeastern Washington.

*5. Irrigation practice on the Great Plains, by E. B. Cowgill. 1897. 39 pp., 12 pls.

Contains directions for constructing reservoirs for pumped well water (pp. 14-19).

*6. Underground waters of southwestern Kansas, by Erasmus Haworth. 1897. 65 pp., 12 pls. $15 \mathrm{c}$.

Covers a rectangular area comprising all of Meade County, nearly all of Seward, Haskell, and Gray counties, and parts of Ford and Finney counties. Describes the physiography, geology, water supplies, and jrrigation developments of the area. Discusses the waters of the Dakota sandstone and of the Tertiary formations.

*7. Seepage water of northern Utah, by Samuel Fortier. 1897.50 pp., 3 pls. $10 \mathrm{c}$.

Describes the water supplies of Cache Valley and the seepage waters in Ogden Valley. Discusses the loss of ground water by evaporation, transpiration, and seepage.

*8. Windmills for irrigation, by E. C. Murphy. 1897. 49 pp., 8 pls. 10c.

Describes the apparatus and methods used in making tests of windmills during the summer of 1896 in the vicinity of Garden City, Kans. Gives the results of these tests and draws conclusions.

*9. Irrigation near Greeley, Colo., by David Boyd. . 1897. 90 pp., 21 pls.

Describes the water supplies, irrigation developments, and agricultural practice in the valley of Cache la Poudre River, a tributary of the South Platte. Discusses the legislative and judicial control of the water supplies (including ground water), the source and disposal of ground water, the use of ground water, the effects of alkali waters on soil, pumping of ground water, and artesian wells.

*10. Irrigation in Mesilla Valley, N. Mex., by F. C. Barker. 1898. 51 pp., 11 pls. $10 \mathrm{c}$.

Describes primitive methods of irrigation and agriculture employed in the valley of the Rio Grande between Fort Seldon, N. Mex., and El Paso, Tex. Describes pumping for irri- . gation with windmills and steam engines.

*12. Underground waters of a portion of southeastern Nebraska, by N:H. Darton. 1898. 56 pp., 21 pls.

Covers an area that includes Lancaster, Seward, York, Fillmore, Hamilton-Clay, Hall, Adams, Buffalo, Kearney, and Phelps counties and parts of Saline, Gosper, and Dawson counties. Describes the physiography, geology, and ground waters of the area and diseusses briefly the prospects for obtaining water from deep-seated formations. 
*13. Irrigation systems in Texas, by W. F. Hutson. 1898.68 pp., 10 pls.

Describes and discusses irrigation works and projects in Texas, considering both surface and ground waters as sources of supply. Superseded by Water-Supply Paper 71.

*14. New tests of certain pumps and water lifts used in irrigation, by O. P. Hood. 1898. $91 \mathrm{pp} ., 1 \mathrm{pl}$. $10 \mathrm{c}$.

Discusses the mechanics and efficiency of reciprocating and of water lifts of various other types.

*17. Irrigation near Bakersfield, Cal., by C. E. Grunsky. 1898. 96 pp., 16 pls.

Describes irrigation with surface water and contains also a statement on early pumping from wells for irrigation at Lindsay, San Joaquin Valley, Cal. (p. 94).

*18. Irrigation near Fresno, Cal., by C. E. Grunsky. 1898, 94 pp., 14 pls. 10c.

Describes irrigation with surface water and contains a brief discussion of the loss of water from Kings River and Fresno Canal and its effect on the water table (pp. 71-79).

*20. Experiments with windmills, by T. O. Perry. 1899. 97 pp., 12 pls. 15c.

Gives data and discusses results of numerous laboratory experiments with wind-driven wheels. Describes the apparatus and methods used.

*21. Wells of northern Indiana, by Frank Leverett. 1899. 82 pp., 2 pls.

Gives well data by counties for the northern part of the State and contains two maps of Indiana and western Ohio, showing the character and depth of the glacial drift and its relation to water supplies.

*25. Water resources of the State of New.York, Part II, by G. W. Rafter. 1899. pp. 105-200, 12 pls. 15c.

Discusses chiefly water storage and power and canal projects but also contains data in regard to seepage from canals (pp. 159-160, 173-178) and quantities of water yielded by the sand deposits of Long Island (pp. 191-198).

*26. Wells of southern Indiana, by Frank Leverett. 1899. 64 pp. $5 c$. Gives well data by counties for the southern part of the State.

27. (See p. 47.)

28. (See p. 47.)

*29. Wells and windmills in Nebraska, by E. H. Barbour. 1899. 85 pp., 27 pls. $15 \mathrm{c}$.

Describes home-made windmills, various other water-lifting devices, salt-water wells, and blowing and freezing wells; gives well data and promiscuous information in regard to ground water.

*30. Water resources of the Lower Peninsula of Michigan, by A. C. Lane. 1899. 97 pp., 7 pls.

Discusses the use of water and the effects of quality with respect to the various uses. Describes the geology and ground-water conditions in the area covered.

*31. Lower Michigan mineral waters, by A. C. Lane. 1899.' 97 pp., 4 pls. $10 \mathrm{c}$.

Discusses the economic value of mineral watprs and the interpretation and classification of water analyses; contains analyses of water from various geologic formations and generalizations in regard to them; also discusses sanitary conditions of drinking waters.

*34. Geology and water resources of a portion of southeastern South Dakota, by J. E. Todd. 1900.34 pp., 10 pls. $10 \mathrm{c}$.

Covers a rectangular area that includes parts of Turner, Hutchinson, Bonhomme, Yankton, and Clay counties. Describes the geology and the surface and ground waters with special reference to the artesian water in the Dakota sandstone. Contains a geologic map and maps showing depths to bed rock, depths to the water at the base of the till, and depths to the artesian water. This area is also covered by Geologic Folios 96 and 97 .

36. (See pp. 47-48.)

37. (See p. 48.)

39. (See p. 48.) 
*41. The windmill; its efficiency and economic use, Part I, by E. C. Murphy. 1901. 72 pp., 14 pls. $5 \mathrm{c}$.

See under Watér-Supply Paper 42.

*42. The windmill; its efficiency and economic use, Part II, by E. C. Murphy. 1901. pp. $77-147,2$ pls. 10c.

Nos. 41 and 42 give a classification of windmills, describe early experiments with windmills and tests made by the writer, describe the apparatus and methods used in making these tests, and discuss the results of the tests.

*45. Water storage on Cache Creek, Cal,, by A. E. Chandler. 1901. 48 pp., 10 pls. $15 \mathrm{c}$.

Contains data in regard to wells and irrigation with ground water near Woodland, Sacramento Valley, Cal. (pp. 23-26).

50. Operations at river stations, 1900, Part IV. 1901. $96 \mathrm{pp}$.

Includes a section on "Computations of seepage in Colorado," pp. 299-306, in which are given data on seepage, chiefly seepage into rivers, and conclusions as to the relation of irrigation to seepage.

52. Operations at river stations, 1900, Part VI. 1901. pp. 495-575.

Contains a section entitled "Construction of wells in southern California" (pp. 497-498).

*53. Geology and water resources of Nez Peree County, Idaho, Part I, by I. C. Russell. 1901. 86 pp., 10 pls. 10c.

See under Water-Supply Paper 54.

*54. Geology and water resources of Nez Perce County, Idaho, Part II, by I. C. Russell. 1901. Pages 87-141.

Nos. 53 and 54 relate to an indeflnite area in western Idaho including a part of Nez Perce County and to adjacent areas of Washington and Oregon. They describe briefly the physiography, geology, soils, water supplies, and mineral resources of the region.

The information on ground water, all of which is given in Water-Supply Paper 54, includes meager data with recommendations in regard to springs, "horizontal wells" or infiltration tunnels, and artesian wells. No. 54 also contains a short bibliography of artesian waters.

55. Geology and water resources of a portion of Yakima County, Wash., by G. 0 . Smith. 1901. 68 pp., 7 pls. 10c.

Describes the geography, geology, and surface and ground waters of an area comprising about 50 square miles in the vicinity of Yakima. Discusses the artesian basins in detail and gives well data. The part of this area west of Yakima is also covered by Geologic Folio 86, which contains a more detailed geologic map and also contains a discussion of the artesian and other ground-water conditions.

*57. Preliminary list of deep borings in the United States, Part I (Alabama-Montana), by N. H. Darton. $1902.60 \mathrm{pp}$. $5 \mathrm{c}$.

See under Water-Supply Papers 61 and 149.

*58. Storage of water on Kings River, Cal., by J. B. Lippincott. 1902.101 pp., 32 pls. $15 \mathrm{c}$.

Contains discussions of ground water for irrigation and of alkali conditions and includes records of 854 wells (pp. 22-24, 53-88). Contains map (Pl. V) showing locations of wells listed in the table.

*59. Development and application of water near San Bernardino, Colton, and Riverside, Cal., Part I, by J. B. Lippincott. 1902. 95 pp., 11 pls.

- See under Water-Supply Paper 60.

*60.' Development and application of water near San Bernardino, Colton, and Riverside, Cal., Part II, by J. B. Lippincott. 1902. pp. 96-141. $15 \mathrm{c}$.

Nos. 59 and 60 give descriptions of pumping plants and of ground-water supphes. No. 60 contains records of 412 wells in Redlands quadrangle and of 478 wells in San Bernardino quad. rangle. No. 59 includes a topographic map showing contours of water table, areas of artesian flow in 1897 and 1960, irrigated areas and locations of wells for which records are given. 
*61. Preliminary list of deep borings in the United States, Part II (NebraskaWyoming), by N. H. Darton. 1902. 67 pp. 5c.

Nos. 57 and 61 contain tabular data in regard to wells and other borings more than 400 feet deep. They give information as to the depths, diameters, and yields of the wells, the head, temperature, and quality of the water, and purposes for which the boring was done. The data are given by States, and the States are arranged alphabetically. The States from Alabama to Montana, inclusive, are covered by No. 57, and the States from Nebraska to Wyoming, inclusive, by No. 61. A revised edition for all States was published in 1905 as Water-Supply Paper 149.

62. Hydrography of the southern Appalachian Mountain region, Part I, by H. A. Pressey. 1902. 95 pp., 25 pls.

Contains a paragraph on large springs in the Watauga River basin (p. 82).

*66. Operations at river stations, 1901, Part II. 1902.188 pp. $10 \mathrm{c}$.

Contains a brief description of the springs, flowing wells, and ground-water conditions in the vicinity of Carrizo Springs, Tex. Includes an analysis of the Carrizo Springs mineral water (p. 63).

*67. The motions of underground waters, by C. S. Slichter. $1902.106 \mathrm{pp} ., 8 \mathrm{pls}$. $15 \mathrm{c}$.

Discusses the origin, depth, and amount of ground waters; the porosity and permeability of rocks and soils; the causes, rates, and laws of the movements of ground water, the surficial and deep zones of circulation, the recovery of water by wells, and the shape and position of the water table. Gives simple methods of measuring the yield of fiowing wells. Describes artesian wells at Savannah, Ga.

70. Geology and water resources of the Patrick and Goshen Hole quadrangles, in eastern Wyoming and western Nebraska, by G. I. Adams. 1902.50 pp., 11 pls. $15 \mathrm{c}$.

Describes the geology and contains some information on springs and wells in these quadrangles.

71. Irrigation systems of Texas, by T. U. Taylor. 1902.137 pp., 9 pls. 10c.

Discusses the principal irrigation systems, giving special attention to the irrigation of rice. Contains data in regard to numerous springs and artesian and other wells.

75. Report of progress of stream measurements for the calendar year 1901, by F. H. Newell. 1903.246 pp., 13 pls.

Contains, on pages 204 and 205, records of discharge of 33 flowing wells in Moxie Valley, near Yakima, Wash.

77. The water resources of Molokai, Hawaiian Islands, by Waldemar Lindgren. 1903. 62 pp., 4 pls. $10 \mathrm{c}$.

Describes the geography, geology, and water resources of the island of Molokai, including springs and wells. Describes the occurrence of water in lava and its quality as affected by the sea. Contains a discussion and estimate of the quantity of ground water available for irrigation.

*78. Preliminary report on artesian basins in southwestern Idaho and southeastern Oregon, by I. C. Russell. 1903.53 pp., 2 pls. 5 c.

Describes briefly the geology of a part of the Snake River plains in Canyon and Owyhee counties, Idaho, and Malheur and,Harney counties, Oreg. Discusses the conditions on which artesian flow depends and describes springs and wells in the Lewis, Otis, Harney, and Whitehorse artesian basins. Describes artesian wells in alluvial deposits and discusses the size of drill holes, the methods of casing, the preservation of well records, and the importance of laws to govern the use of artesian waters. Gives a list of publications bearing on artesian waters.

84. Report of progress of stream measurements for the calendar year 1902, by F. H.

- Newell, Part III, Western Mississippi River and western Gulf drainage. 1903. $200 \mathrm{pp}$.

Contains a brief description of Barton Springs, near Austin, Tex. (pp. 152, 153). See also Water-Supply Papers 132 and 174.

85. (Seee p. 48.) 
89. Water resources of the Salinas Valley, Cal., by Homer Hamlin. $1904.91 \mathrm{pp}$,, $12 \mathrm{pls}$. $15 \mathrm{c}$.

Includes data in regard to ground water and pumping plants and a map showing the area of artesian flow and the areas irrigated with water obtained from wells.

90. Geology and water resources of part of the lower James River valley, S. Dak., by J. E. Todd and C. M: Hall. 1904. 47 pp., 23 pls. $35 \mathrm{c}$.

Describes the geology, surface waters, and artesian and other ground waters of a rectangular area comprising Davison, Hanson, Sanborn, Beadle, and Miner counties and parts of Kingsbury, Jerauld, Aurora, and McCook counties. Includes a geologic map of the area and maps showing areas of artesian flow, depths to Dakota sandstone, head of artesian water, depths to bed rock, and depths to water at the base of the till. The area is also described in Geologic Folios 99, 100, 113, and 114.

91. The natural features and economic development of the Sandusky, Maumee, Muskingum, and Miami drainage areas in Ohio, by B. H. and M. S. Flynn. 1904. $130 \mathrm{pp}$. $10 \mathrm{c}$.

Includes descriptions of numerous public water supplies obtained from wells and springs (pp. 58-124).

98. (See p. 48.)

*99. Report of progress of stream measurements for the calendar year 1903. Part III, Western Mississippi River and western Gulf of Mexico drainage, by J. C. Hoyt. 1904. 422 pp., 1 pl. 25c.

Contains on pages 235-237 a description and discharge record of Meramee Spring, near Meramec, Mo. See also Water-Supply Papers 131, 173, and 209. Contains, on page 321, the discharge records of 16 springs in Oklahoma.

101. Underground waters of southern Louisiana, by G. D. Harris, with discussions of their uses for water supplies and for rice irrigation, by M. L. Fuller. 1904. 98 pp., 11 pls. $20 \mathrm{c}$.

Describes the geology and ground-water conditions of the area, gives data in regard to artesian wells, and outlines methods of well drilling, pumping, and rice irrigation. Includes 23 analyses of ground water.

102. Contributions to the hydrology of eastern United States, 1903; M. L. Fuller, geologist in charge. $1904.522 \mathrm{pp} .30 \mathrm{c}$.

Contains a list of publications of the United States Geological Survey relating to ground water, with special reference to springs. Gives an account of the organization and of the work of the division of hydrology (ground water). Contains notes on wells, springs, and general water resources, arranged by counties, in the following States:

Maine, by W. S. Bayley. Contains records of 224 wells and 130 springs and analyses of 3 well waters and of 3 spring waters (pp. 27-55).

New Hampshire, by J. M. Boutwell. Contains records of 35 wells and 107 springs and analyses of 9 well waters and 12 spring waters (pp. 56-72).

Vermont, by G. H. Perkins. Contains a table giving data in regard to the water supplies of Vermont towns, contains also 111 partial analyses of waters from wells, springs, streams, and lakes and records of 44 wells and 90 springs ( $\mathrm{pp}$. 73-93).

Massachusetts, by W. O. Crosby and Laurence LaForge. Contains records of 162 wells and 40 springs and analyses of 7 well waters and 17 spring waters (pp. 94-118).

Rhode Island, by W. O. Crosby. Contains records of 24 wells and 12 springs and analyses of 2 well waters and 3 spring waters (pp. 120-126).

Connecticut, by H. E. Gregory. Contains records of about 470 wells and 84 springs and analyses of 19 well waters and 19 spring waters (pp. 127-168).

New York, by F. B. Weeks. Contains records of 371 wells and 228 springs and analyses of 14 well waters (pp. 169-206).

Georgia, by S. W. McCallie. Contains a table giving data in regard to water supplies of cities and villages in the State. Contains records of 90 wells and 100 springs and a table giving the yields of 14 springs. Includes analyses of water from 1 well and 1 spring (pp. 207-237).

Florida, by $M$. L. Fuller. Contains records of 242 wells and 43 springs and analyses of 11 well waters and 9 spring waters. Gives the yields of some very large springs (pp. 238-275).

Alabama, by E. A. Smith. Contains notes on numerous wells by counties (pp. 276-331). Mississippi, by L. C. Johnson and E. C. Eckel. Contains records of 265 wells (pp. 332-357). Tennessee, by L. C. Glenn. Contains records of 78 wells (pp. 357-367). 
102. Contributions to the hydrology of eastern United States, 1903-Continued.

Kentucky, by L. C: Glenn. Contains records of 16 wells and 2 analyses of artesian water (pp. 369-373).

Arkansas, by A. H. Purdue. Contains records of 96 wells and 33 springs and analyses of 3 well waters (pp. 374-388).

Missouri, by E. M. Shepard. Contains records of 105 wells and 112 springs and analyses of 13 well waters and 34 spring waters (pp. 389-440). Inoludes brief papers on wells of Joplin and vicinity, by W. S. T. Smith (pp. 404-409), and the water supply of Livingston County, by R. Hawkins (pp. 410-416).

Minnesota, by C. W. Hall. Contains records of about 800 shallow wells, about 200 deep wells, and 76 springs; includes an analysis of water from a spring at Mankato (pp. 441-488).

Lower Michigan, by W. F. Cooper. Contains records of 198 wells and 31 springs and analyses of 11 well waters and 15 spring waters (pp. 489-512).

104. The underground waters of Gila Valley, Ariz., by W. T. Lee. 1904.71 pp., 5 pls. $10 \mathrm{c}$.

Describes the topography and geology of the Gila Valley between The Buttes, 12 miles east of Florence, and the mouth of Salt River; treats of the source, amount, and quality of water in the valley fill and the methods of recovering this water; includes well data and water analyses.

105. The water powers of Texas, by T. U. Taylor. 1904 . $116 \mathrm{pp}, 17 \mathrm{pls}$.

Gives data regarding Hackberry Springs ( 2 miles northwest of Toyah Lake) and Santa Rosa Spring, noar Santa Lucia, in Pecos County (pp. 14, 15).

106. Water resources of the Philadelphia district, by Florence Bascom. 1904. 75 pp., 4 pls. 5c.

Describes the geology and the streams, springs, wells, and public water supplies of an area comprising the Germantown, Norristown, Philadelphia, and Chester quadrangles. Discusses artesian conditions and prospects in the crystalline rocks, the Triassic formations, and the formations of the Coastal Plajn. The area is also deseribed in Geologic Folio 162, which contains considerable information on ground water.

108. Quality of water in the Susquehanna River drainage basin, by M. O. Leighton, with an introductory chapter on physiographic features, by G. B. Hollister. 1904. 76 pp., 4 pls. $15 \mathrm{c}$.

Contains analyses of ground waters and discussions of the quality of these waters.

110. Contributions to the hydrology of eastern United States, 1904; M. L. Fuller, geologist in charge. 1905.211 pp., 5 pls. 10c.

Contains the reports in the following list. Most of those covering specific areas do not include any maps.

Description of underflow meter used in measuring the velocity and direction of underground water, by Charles S. Slichter (pp. 17-31).

The California or "stovepipe" method of well construction, by Charles S. Slichter (pp. 32-36).

Approximate methods of measuring the yield of flowing wells, by Charles S. Slichter (pp. $37-42)$.

Corrections necessary in accurate determinations of flow from vertical well casings, from notes furnished by A. N. Talbot (pp. 43-44).

Experiment relating to problems of well contamination at Quitman, Ga., by S. W. McCallie (pp. 45-54).

The new artesian water supply at Ithaca, N, Y., by F. L. Whitney (pp. 55-64).

Drilled wells of the Triassic area of the Connecticut Valley, by W. H. C. Pynchon (pp. 65-94).

Triassic rocks of the Connecticut Valley as a source of water supply, by M. L. Fuller (pp. 95-112).

Spring system of the Decaturville dome, Camden County, Mo., by E. M. Shepard (pp. 113-125).

Water resources of the Fort Ticonderoga quadrangle, Vt. and N. Y., by T. N. Dale (pp. 126-129).

Water resources of the Taconic quadrangle, N. Y., Mass., and Vt., by F. B. Taylor (pp. 130-133).

Water resources of the Watkins Glen quadrangle, N. Y., by R. S. Tarr (pp. 134-140). The ground-water conditions of this quadrangle are described more fully in Geologic Folio 169.

Water resources of the central and southwestern highlands of New Jersey, by Laurence La Forge (pp. 141-155). 
110. Contributions to the hydrology of eastern United States, 1904-Continued.

Water resources of the Chambersburg and Mercersburg quadrangles, Pa., by G. W. Stose (pp. 156-158). The ground-water conditions of these quadrangles are described more fully in Geologio Folio 170.

Water, resources of the Curwensville, Patton, Ebensburg, and Barnesboro quadrangles, $\mathrm{Pa}$., by F. G. Clapp (pp. 159-163). The ground-water conditions of the Barnesboro and Patton quadrangles are also briefly described in Geologic Folio 189, and those of the Ebensburg quadrangle in Geologic Folio 133. The ground waters of the Curwensville, Barnesboro, and Patton quadrangles are also briefly described in Bulletin 531-d.

Water resources of the Elders Ridge quadrangle, Pa., by $\mathrm{R}$. W. Stone (pp. 164, 165). The ground-water conditions of this quadrangle are also described in Bulletin 256 (with geologic map) and in Geologic Folio 123.

Water resources of the Waynesburg quadrangle, Pa., by $\mathbf{R}$. W. Stone (pp. 166, 167). The ground-water conditions of this quadrangle are also described in Geologic Folio 121.

Water resources of the Accident and Grantsville quadrangles, Md., by G. C. Martin (pp. 168-170). The ground-water conditions of these quadrangles are also described in Geologic Folio 160 .

Water resources of the Frostburg and Flintstone quadrangles, Md. and W. Va., by G. C. Martin (pp. 171-173).

Water resources of Cowee and Pisgah quadrangles, N. C., by H. S. Gale (pp. 174-176). The ground-water conditions of the Pisgah quadrangle are described also in Geologic Folio 147.

Water resources of the Middleboro-Harlan region of southeastern Kentucky, by G. H. Ashley (pp. 177-178).

Summary of the water supply of the Ozark region in northern Arkansas, by G. I. Adams (pp. 179-182).

Notes on the hydrology of Cuba, by M. L. Fuller (pp. 183-200).

111. Preliminary report on the underground waters of Washington, by Henry Landes. - 1905. 85 pp., 1 pl. $10 \mathrm{c}$. State.

Describes briefly by counties the deep wells, springs, and municipal water supplies of the

*112. Underflow tests in the drainage basin of Los Angeles River, by Homer Hamlin. 1905. 55 pp., 7 pls. $5 \mathrm{c}$.

Describes in detail the methods and apparatus used in making measurements of the rate and volume of underflow and gives the results of underflow tests made in the valley of Los Angeles River in 1902 and 1903.

113. The disposal of strawboard and oil-well wastes, by R. L. Sackett and Isaiain Bowman. 1905. 52 pp., 4 pls. $5 \mathrm{c}$.

The second part of this paper describes briefly the geology and ground waters of the region about Marion, Ind., and the contamination of rock wells and streams by waste oil and brine.

*114. Underground waters of eastern United States; M. L. Fuller, geologist in charge. 1905. 285 pp., 18 pls. $25 \mathrm{c}$.

Contains a paper entitled "Occurrence of underground waters," by M. L. Fuller, in which are discussed the source, quantity, and temperature of ground waters, the permeability and storage capacity of water-bearing formations, the recovery of water through springs and wells, the conditions that produce artesian, flow and the general ground-water conditions in eastern United States (pp. 18-40).

Contains also brief reports on ground water in the following States, each of which includes a bibliography:

Maine, by W. S. Bayley (pp. 41-56). Includes analyses of 35 spring waters.

New Hampshire, by M. L. Fuller (pp. 57-59).

Vermont, by G. H. Perkins (pp. 60-67). Includes a sketch map of the State, showing waterbearing deposits and analyses of 3 spring waters.

Massachusetts and Rhode Island, by W. O. Crosby (pp. 68-75).

Connecticut, by H. E. Gregory (pp. 76-81). Includes a sketch map of the State, showing the rock formations.

New York, by F. B. Weeks (pp. 82-92). Includes a sketch map of the State, showing the rock formations.

New Jersey, by G. N. Knapp (pp. 93-103). Includes a sketch map of the State, showing the ground-water provinces, and 5 geologic sections, showing the water-bearing formations of the Coastal Plain.

Pennsylvania, by M. L. Fuller (pp. 104-110). Includes a sketch map of the State, showing the main geologic systems. 
${ }^{*} 114$. Underground waters of eastern United States-Continued.

Delaware, by N. H. Darton (pp. 111-113). Includes geologic sections showing water-bearing formations.

Maryland, by N. H. Darton and M. L. Fuller (pp. 114-123).. Includes geologic sections showing water-bearing formations.

District of Columbia, by N. H. Darton and M. L. Fuller (pp. 124-126). Includes geologic sections showing water-bearing formations.

Virginia, by N. H. Darton and M. L. Fuller (pp. 127-135). Includes geologic sections showing water-bearing formations.

North Carolina, by M. L. Fuller (pp. 136-139).

South Carolina, by L. C. Glenn (pp. 140-152). Includes geologic sections showing waterbearing formations.

Georgia, by S. W. McCallie (pp. 153-158). Includes a sketch map of the State, showing areas of artesian flow.

Florida, by M. L. Fuller (pp. 159-163).

Alabama, by E. A. Smith (pp. 164-170). Includes a sketch map of the State, showing groundwater conditions.

Mississippi, by L. C. Johnson (pp. 171-178). Includes a geologic sketch map of the State.

Louisiana and southern Arkansas, by A. C. Veatch (pp. 179-187). Includes sketch maps showing ground-water conditions.

Northern Arkansas, by A. H. Purdue (pp. 188-197). Includes a geologic sketch map and sections of the area.

Tennessee and Kentucky, by L. C. Glenn (pp. 198-208).

Missouri, by E. M. Shepard (pp. 209-219). Includes sketch maps showing the geology and and ground-water conditions of the State.

Iowa, by W. H. Norton (pp. 220-225).

Minnesota, by C. W. Hall (pp. 226-232).

Wisconsin, Northern Peninsula of Michigan and the portion of Mlinois north of the Carboniferous deposits, by Alfred R. Schultz (pp. 232-241). Includes a sketch map of the region showing "the outcrops of the "Potsdam" and St. Peter sandstones.

Lower Michigan, compiled from report by A. C. Lane (pp. 242-247). Includes a geologic sketch map and a section of the area.

Illinois, by Frank Leverett (pp. 248-257). Includes a geologic sketch map and a section of the State.

Indiana, by Frank Leverett (pp. 258-264). Includes sketch maps of the State, showing the geology, the depth of the glacial drift, and the relation of wells to depths of drift.

Ohio, by Frank Leverett (pp. 265-270). Includes sketch maps of the State showing the geol$\mathrm{ngy}$, the depths of the glacial drift, and the relation of wells to the depth of drift.

West Virginia, by M. L. Fuller (pp. 271-272).

116. Water problems of Santa Barbara, Cal., by J. B. Lippincott. 1905. 99 pp., 8 pls. 10c.

Deals chiefly with surface waters but contains data on deep city wells and collecting tunnel and analysis of tunnel water (pp. 33-42,57).

118. Geology and water resources of a portion of east-central Washington, by F. C. Calkins. 1905. 96 pp., 4 pls. 5c.

Describes briefly the geology of the Columbia Plains and the Kittitas Valley, gives information in regard to the streams, springs, and wells, and discusses the artesian prospects.

120. Bibliographic review and index of papers relating to underground waters published by the United States Geological Survey, 1879-1904, by M. L. Fuller. 1905. $128 \mathrm{pp}$. $10 \mathrm{c}$.

Lists all papers that contain information on ground water. Gives brief abstracts of these papers in regard to ground water, with page references for papers dealing mainly with other subjects. Contains an index of the papers listed in so far as they relate to ground water.

*122. Relation of the law to underground waters, by D. W. Johnson. $1905.55 \mathrm{pp}$. $5 c$.

Defines and classifies ground waters and gives common-law rules and State legislative acts relating to their use.

*123. Geology and underground water conditions of the Jornada del Muerto, N. Mex., by C. R. Keyes. 1905.42 pp., 9 pls. $15 \mathrm{c}$.

This report is superseded by Water-Supply Paper 188. 
127. (See p. 48.)

130. (See p. 48.)

131. Report of progress of stream measurements for the calendar year 1904, Part VIII, Platte, Kansas, Meramec, Arkansas, and Red River drainages, by M. C. Hinderlider and J. C. Hoyt. 1905.

Contains, on pages 123-125, a description and discharge record for Meramec Spring, near Meramec, Mo. See also Water-Supply Papers 99, 173, and 209.

132. Report of progress of stream measurements for the calenäar year 1904, Part IX, Western Gulf of Mexico and Rio Grande drainages, by T. U. Taylor and J. C. Hoyt. 1905. 132 pp., 2 pls.

Contains descriptions of the following springs in Texas: Lipan and Kickapoo springs near San Angelo (p. 43); Barton and Mormon springs, near Austin (pp. 44 and 45); Toyah Spring, at Toyahville (pp. 121 and 122); and Santa Rosa Spring near Fort Stockton (p. 122). (See also p. 127.)

133. (See p. 48.)

135. (See p. 48.)

136. Underground waters of Salt River valley, Ariz., by W. T. Lee. $1905 . ' 196$ pp., 23 pls. 25 c.

Describes the geology of that part of the valley in which Phoenix and Mesa are situated. Gives well records and discusses the quantity and chemical character of the ground waters, the duty of water for irrigation, and the cost of pumping. Contains maps showing the geology and the position of the warer table.

*137. Development of underground waters in the eastern coastal-plain region of southern California, by W. C. Mendenhall. 1905. 140 pp., 7 pls. 35c.

Describes the ground-water conditions and theirrigation systems in the Anaheim and Santa Ana quadrangles in Los Angeles and Orange counties. Discusses the effects of development and drought on ground-water levels, contains records of 2,765 wells, and includes maps showing original areas of artesian flow, areas of artesian flow in 1904, ground-water levels, irrigated lands, and locations of wells and pumping plants. Includes also a general map of the "Valley of southern California," showing contours of the water table, original areas of artesian flow, and areas of artesian flow in 1904.

*138. Development of underground waters in the central coastal-plain region of southern California, by W. C. Mendenhall. 1905. 162 pp., 5 pls. 25c. See under Water-Supply Paper 139.

Describes the ground-water conditions and the irrigation systems in the Downey and Las Bolas quadrangles, in Los Angeles and Orange counties. Discusses the effects of development and drought on ground-water levels, contains records of 3,323 wells, and inchudes maps showing original aréas of artesian flow, areas of artesian flow in 1904, ground-water levels, irrigated lands, and locations of wells and pumping plants. Contains also the general map mentioned under Water-Supply Paper 137.

*139. Development of underground waters in the western coastal-plain region of southern California, by W. C. Mendenhall. 1905.105 pp., 8 pls. $25 \mathrm{c}$.

Describes the ground-water conditions and theirrigation systems in the Santa Monica and Redondo quadrangles, in Los Angeles County. Discusses the effects of development and drought on changes in ground-water levels, contains records of 2,097 wells, and includes maps showing original areas of artesian flow, areas of artesian flow in 1904, ground-water levels, irrigated lands, and locarioas of wells and pumping plants. Contains also thegeneral map mentioned under Water-Supply Paper 137.

140. Field measurements 'of the rate of movement of underground waters, by C. S.

Slichter. $1905.122 \mathrm{pp} ., 15 \mathrm{pls}$. $15 \mathrm{c}$.

Discusses the capacity of sand to transmit water, describes the under-flow meter deviseI by the author and laboratory experiments on the flow of water through sands and gravels, and gives results of measurements of underflow in Rio Hondo, San Gabriel, and Mohave River valleys, Cal., and on Long Island, N. Y. Discusses specific capacities of wells, gives results of tests of wells and pumping plants in the Rio Grande valley in New Mexico and Texas and the Arkansas Valley in Kansas, and describes the "stovepipe" mothod of well constructution. 
141. Observations on the ground waters of the Rio Grande valley, by C. S. Slichter. 1905. 83 pp., 5 pls. $5 \mathrm{c}$.

Describes investigation of the underflow in the valley of the Rio Grande in Texas and New Mexico, gives details of tests of pumping plants near El Paso, Tex., in Mesilla Valley, N. Mex., and near Berino, N. Mex., and gives analyses of well waters and data concerning wells at and near E1 Paso.

*142. The hydrology of San Bernardino Valley, Cal., by W. C. Mendenhall. 1905. 124 pp., 12 pls. $25 \mathrm{c}$.

Describes the source, circulation, quantity, temperature, and chemical character of the ground water, gives records of 890 wells, and contains maps showing changes in areas of artesian flow and in ground-water levels, and locations of wells, pumping plants, and irrigated lands. This paper, like Nos. $137,138,139$, and 219 , also contains a general map of the "Valley of southern California," showing contours of water table, original areas of artesian flow, and areas of artesian flow in 1904.

144. The normal distribution of chlorine in the natural waters of New York and New England, by D. D. Jackson. 1905. 31 pp., 5 pls. $10 \mathrm{c}$.

Discusses the content of sodium chloride in coast and inland waters and its value in indicatingpollution of streams and wells. Describes thesolutionsand methods used in determinations of chlorine. Contains maps showing the normal distribution of chlorine in surface and ground waters in the New England States and New York, and tables giving data on which the maps are based.

145. Contributions to the hydrology of eastern United States, 1905; M. L. Fuller, geologist in charge. 1905 . 220 pp., 6 pls. 10c.

Contains the reports in the following list. Most of those covering specified areas do not include any maps.

Hydrologic work in eastern United States and publications on ground waters, by $\mathbf{M} . \mathbf{L}$. Fuller (pp. 9-29).

The drainage of ponds into drilled wells, by R. E. Horton (pp. 30-39).

Two unusual types of artesian flow, by M. L. Fuller (pp. 40-45).

Construction of so-called fountain and geyser springs, by M. L. Fuller (pp. 46-50).

A convenient gage for determining low artesian heads, by M. L. Fuller (pp. 51, 52).

Water resources of the Catatonk area, N. Y., by E. M. Kindle (pp. 53-57). The ground-water conditions in this quadrangle are also described in Geologic Folio 169.

Water resources of the Pawpaw and Hancock quadrangles, W. Va., Md., and Pa., by G. W. Stose and G. C. Martin (pp. 58-63). The ground-water conditions in these quadrangles are also described in Geologic Folio 179.

Water resources of the Nicholas quadrangle, W. Va., by G. H. Ashley (pp. 64-66).

Water resources of the Mineral Point quadrangle, Wis., by U. S. Grant (pp. 67-73). The ground-water conditions in this quadrangle are also described in Geologic Folio 145.

Water resources of the Joplin district, Mo.-Kans., by W. S. T. Smith (pp. 74-83). The ground-water conditions in this quadrangle are also described in Geologic Folio 148, but analyses are given only in the water-supply paper.

Water resources of the Winslow quadrangle, Ark., by A. H. Purdue (pp. 84-87). The groundwater conditions in this quadrangle are also described in Geologic Folio 154.

Water resources of the contact region between the Paleozoic and Mississippi embayment deposits in northern Arkansas, by A. H. Purdue (pp. 88-119).

Water resources of the Portsmouth-York region, N. H. and Maine, by G. O. Smith (pp. 120,128 ).

A ground-water problem in southeastern Michigan, by M. L. Fuller (pp. 129-147).

Water supplies at Waterloo, Iowa, by W. H. Norton (pp. 148-155).

Water supply from glacial gravels near Augusta, Maine, by G. O. Smith (pp. 156-160).

Water-supply from the delta type of sand plain, by W. O. Crosby (pp. 161-178).

Waters of a gravel-filled valley near Tully, N. Y., by G. B. Hollister (pp. 179-184).

Notes on certain hot springs of the southern United States, by W. H. Weed (pp. 185-206).

Notes on certain large springs of the Ozark region, Mo. and Ark., compiled by M. L. Fuller (pp. 207-210). 
146. Proceedings of second conference of engineers of the Reclamation Service, with accompanying papers, compiled by F. H. Newell, chief engineer. 1905. $267 \mathrm{pp}$. $15 \mathrm{c}$.

Contains a brief account of the organization of the hydrographic [water resources] branch, including the division of hydrology [ground water]. Includes the following papers relating to ground water, drilling methods, and pumping for irrigation.

Pumping underground water in southern California, by F. C. Finkle.

Diamond-drill methods, by G. A. Hammond.

Underground waters of southern California, by W. C. Mendenhall.

Cost of power for pumping irrigating water, by $H$. A. Storrs.

*148. Geology and water resources of Oklahoma, by C. N. Gould. 1905. 178 pp., 22 pls. 20c.

Covers only the original Territory of Oklahoma, not the eastern part of the State. Describes the topography and geology and the streams, springs, and wells; discusses artesian conditions, outlines the water supplies by counties; treats of irrigation from reservoirs, springs, and wells; and gives 154 analyses of well waters and a table containing records of 261 wells. Includes a geologic map.

149. Preliminary list of deep borings in the United States, second edition with additions, by N. H. Darton. $1905.175 \mathrm{pp} .10 \mathrm{c}$.

Gives location, depth, diameter, yield, water level, and other available information concerning wells 400 feet or more in depth; includes all wells listed in Water-Supply Papers 57 and 61. Contains some data in regard to every State in the United States, arranged alphabetically by States and counties. Mentions principal publications relating.to deep borings.

*150. Weir experiments, coefficients, and formulas, by R. E. Horton. $1906.189 \mathrm{pp}$., 38 pls. $15 \mathrm{c}$.

Superseded by Water-Supply Paper 200.

*151. Field assay of water, by M. O. Leighton. 1905.77 pp., 4 pls. 10c.

Describes apparatus, reagents, and methods for rapid field determinations of turbidity, color, iron, hardness, carbonates, bicarbonates, sulphates, chlorides, and calcium in water.

152. A review of the laws forbidding pollution of inland waters in the United States (second edition), by E. B. Goodell. 1905. 149 pp. 10c.

Includes laws relating to wells and springs.

*153. The underflow in Arkansas Valley in western Kansas, by C. S. Slichter. 1906. 90 pp., 3 pls. $15 \mathrm{c}$.

Discusses the origin and extent of the underflow, the fluctuation of ground-water level, and the chemical composition of the water. Gives results of underflow measurements and tests of the rate of evaporation of ground water. Gives summaries and details of pumping tests and analyses of river and well waters.

*154. The geology and water resources of the eastern portion of the Panhandle of Texas, by C. N. Gould. 1906.64 pp., 15 pls. $10 \mathrm{c}$.

Describes the topography and geology and the streams and springs, discusses the groundwater conditions and irrigation, and gives detailed data by counties. Includes a geologic map.

*155. Fluctuation of the water level in wells, with special reference to Long Island, N. Y., by A. C. Veatch. 1906.83 pp., 9 pls. $25 \mathrm{c}$.

Gives data on ground-water levels and discusses fiuctuations due to rainfall, evaporation, barometric changes, temperature changes in rivers, changes in lake levels, tidal changes, irriggtion, the construetion of dams, ground-water developments, deforestation, cultivation of the soil, drainage, and other causes.

*157. Underground water in the valleys of Utah Lake and Jordan River, Utah, by - G. B. Richardson. 1906. 81 pp., 9 pls. $20 \mathrm{c}$.

Describes the geology and the ground-water conditions and gives numerous well records. Includes maps showing depths to ground water and areas of artesian fiow.

$56122^{\circ}-18-$ wsP $427-2$ 
158. Preliminary report on the geology and underground waters of the Roswell artesian area, N. Mex., by C. A. Fisher. 1906.29 pp., 9-pls. $15 \mathrm{c}$.

Describes the geology and ground-water conditions of the artesian basin which extends along Pecos River from Roswell to Lake McMillan. Discusses the area and extent of the artesian basin, the source, quantity, pressure, quality, and conservation of the artesian water, and the irrigation with this water. Gives well records and analyses and contains maps showing the area of artesian flow and the intake area.

159. Summary of the underground-water resources of Mississippi, by A. F. Crider and L. C. Johnson. 1906. 86 pp., 6 pls. $20 \mathrm{c}$.

Describes the geology and the ground-water conditions of the State. Gives notes on wells by counties, records of deep wells, and chemucal analyses. Includes a geologic map (P1.I) and a map showing ground-water conditions (Pl. V).

*160. Underground-water papers, 1906; M. L. Fuller, geologist in charge. 1906.104 pp., 1 pl.

Gives an account of work done in $\mathbf{1 9 0 5}$ and lists of publications relating to ground waters; also contains the following reports:

Significance of the term "artesian," by M. L. Fuller (pp. 9-15).

Representation of wells and springs on maps, by M. L. Fuller (pp. 16-18).

Occurrence of w ater in crystalline rocks, by E. E. Ellis (pp. 19-28).

Flowing-well districts in the eastern part of the northern peninsula of Michigan, by Frank Leverett (pp. 29-53).

Drainage of wet lands in Arkansas by wells, by A. F. Crider (pp. 54-58).

Total amount of free water in earth's crust, by M. L. Fuller (pp. 59-72).

Use of fluorescein in the study of underground waters, by R. B. Dole (pp. 73-85).

Peculiar mineral waters from crystalline rocks of Georgia, by M. L. Fuller (pp. 86-91).

Problems of water contamination, by Isaiah Bowman (pp. 92-95).

Instances of improvement of water in wells, by M. L. Fuller (pp. 96-100).

*163. Bibliographic review and index of underground-water literature published in the United States in 1905, by M. L. Fuller, F. G. Clapp, and B. L. Johnson. 1906. $130 \mathrm{pp}$. $15 \mathrm{c}$.

Lists practically all papers that contain any information on ground water; includes brief abstracts with respect to ground water, giving page references for reports dealing mainly with other subjects; contains index with respect to ground-water subjects of papers listed.

*164. Underground waters of Tennessee and Kentucky west of Tennessee River and of an adjacent area in Illinois, by L. C. Glenn. 1906.173 pp., 7 pls. $25 \mathrm{c}$.

Describes the topography and geology, discusses mineral waters and artesian conditions, and outlines the water resources by courties. Contains maps showing the geology and the head of the artesian water.

168. (See p. 48.)

170. Report of progress of stream measurements for the calendar year 1905, Part VI, Great Lakes and St. Lawrence River drainages, by R. E. Horton, F. W. Hanna, and J. C. Hoyt. 1906. 116 pp., 1 pl.

Contains, on pages 24 and 25, a description and a one-year record of discharge of Reeds Springs, near Albibn, Mich.

173. Report of progress of stream moasurements for the calendar year 1905, Part IX, Meramec, Arkansas, Red, and lower western Mississippi River drainages, by M. C. Hinderlider, J. M. Giles, and J. C. Hoyt. 1906. 105 pp., 1 pl.

Contains, on page 17, a description and discharge record of Meramec Spring, near Meramec, Mo. See also Water-Supply Papers 99, 131, and 209.

174. Report of progress of stream measurements for the calendar year 1905, Part X, western Gulf of Mexico and Rio Grande drainages, by T. U. Taylor and J. C. Hoyt. 1906. 113 pp., 1 pl.

Describes Bartons Springs, near Austin, Tex. (pp. 30, 31).

177. (See p. 48.) 
*181. Geology and water resources of Owens Valley, Cal., by W. T. Lee. 1906.28 pp., 6 pls. 15c.

Outlines the geology and ground-water conditions, gives well records, and discusses briefly the artesian prospects, the utilization of ground waters by installation of pumping and power plants, reservoir sites, and the significance of undrained lakes as registers of climate. See also Water-Supply Paper 294.

*182. Flowing wells and municipal water supplies in the southern portion of the southern peninsula of Michigan, by Frank Leverett and others. 1906. 292 pp., 5 pls. $50 \mathrm{c}$.

See under Water-Supply Paper 183.

*183. Flowing wells and municipal water supplies in the middle and northern portions of the southern peninsula of Michigan, by Frank Leverett and others. 1907. 393 pp., 5 pls. $50 \mathrm{c}$.

Nos. 182 and 183 describe in general the geology and ground-water conditions of the areas covered and give details by counties concerning flowing wells and municipal supplies. They . contain numerous analyses and several geologic and artesian-water maps.

*184. The underflow of the South Platte Valley, by C. S. Slichter and H. C. Wolff. 1906. 42 pp. 5c:-

Describes investigations of velocity, direction, and quantity of underflow at Ogalalla, Nebr. gives chemical analyses of the water, and discusses disadvantages of underflow canals or infiltration ditches. Describes also investigations at North Platte, Nebr., and gives suggestions for the construction of small pumping plants.

*188. Water resources of the Rio Grande Valley, N. Mex., and their development, by W. T. Lee. 1907. 59 pp., 10 pls. 20c.

Describes the topography and geology, reservoir sites, and the water resources of the valley. Gives the well data and outlines the ground-water conditions in the Santa Fe, Albuquerque, Belen, Jornada, La Mesa, and Mesilla districts. Discusses the origin, course, and quantity of the ground water, its character, and its utilization by means of wells and infiltration ditches. Contains several analyses of river and well waters and includes a topographic map of Mesilla Valley, showing depths to ground water.

*190. Underground waters of the Coastal Plain of Texas, by T. U. Taylor. 1907. 73 pp., 3 pls. $15 \mathrm{c}$.

Describes the ground waters by counties; gives many well records and analyses; includes a map showing locations of artesian wells.

*191. The geology and water resources of the western portion of the Panhandle of Texas, by C. N. Gould. 1907. 70 pp., 7 pls. $15 \mathrm{c}$.

. Describes the topography and geology, the springs, streams, and shallow and deep-seated ground waters, and the utilization of the waters for irrigation. Gives detailed information by counties and includes a geologic map.

*193. The quality of surface waters in Minnesota, by R. B. Dole and F. F. Wesbrook. 1907. 171 pp., $7 \cdot$ pls. $25 \mathrm{c}$.

Relates chiefly to surface waters but contains scattered notes on wells used for public supplies throughout the State, a table of data in regard to these wells (pp. 146-149), and a brief discussion of the comparative value of surface and ground waters (pp. 151-153).

*195. Underground waters of Missouri, their geology and utilization, by E. M. Shepard. 1907. 224 pp., 6 pls. 30c.

Describes the topography and geology of the State and discusses the water supplies by dis. tricts and counties; gives statistics of city water supplies, analyses of water, and many well sections and records; includes a map of the State, showing the locations of flowing and nonflowing deep wells.

197. (See p. 48.) 
*199. Underground water in Sanpete and central Sevier valleys, Utah, by G. B. Richardson. 1907. 63 pp., 6 pls. $25 \mathrm{c}$.

Describes the geology of the area and the sources, distribution, recovery, and quality of the ground waters; gives detailed descriptions and tabulated data concerning springs and wells includes a map showing the geology and the depths to ground water.

*200. Weir experiments, coefficients, and formulas, by R. E. Horton. 1907. 195 pp., 38 pls. $35 \mathrm{c}$.

Revision of Water-Supply Paper 150. A treatise on the theory of weirs, with tables and curves based on experiments.

*209. Surface water supply of lower western Mississippi River drainage, 1906, by R. I. Meeker and J. M. Giles. 1907. 79 pp., 2 pls. $15 \mathrm{c}$.

Contains, on pages 21 and 22, a description and discharge record of Meramec Spring, near Méramec, Mo. See also Water.Supply Papers 99, 131, and 173. Contains, on page 74, discharge records of Antelope, Buffalo, and Sulphur springs, near Sulphur, Okla.

212. (See pp. 48-49.)

*213. Surface water supply of California, 1906, by W. B. Clapp, with a section on ground-water levels in southern California, by W. C. Mendenhall. 1907. 219 pp., 4 pls. $25 \mathrm{c}$.

Gives the results of a series of measurements of water levels in wells made during 1904, 1905, and 1906 (pp. 189-205). Wells widely distributed over the various basins of southern California were selected in order that the data would give a basis for conclusions as to the fluctuations of the water table in each of these basins.

214. (See p. 49.)

*215. Geology and water resources of a portion of the Missouri River valley in northeastern Nebraska, by G. E. Condra. 1908. 59 pp., 11 pls. $40 \mathrm{c}$.

Describes the geology, streams, springs, and shallow and artesian wells in Boyd, Knox, Cedar, Dixon, and Dakota counties, and in a part of Holt County; includes maps showing the geology and the artesian-water conditions; contains information on "blowing wells."

*216. Geology and water resources of the Republican River valley and adjaceni areas, Nebraska, by G. E. Condra. 1907. 71 pp., 13 pls. $15 \mathrm{c}$.

Describes the geography, geology, and surface and ground waters in Dundy, Hitchcock, Redwillow, Furnas, Harlan, Franklin, Webster, Nuckolls, Thayer, and Jefferson counties; includes a geologic map; contains information on "blowing wells."

*217. Water resources of Beaver Valley, Utah, by W. T. Lee. 1908. 57 pp., 1 pl. 10c.

Describes the geography, geology, streams, springs, and seepage waters of the eastern part of Beaver County. Gives data in regard to deep wells in the Beaver, Greenville, Adamsville, Minersville, and Milford districts, and at railroad stations between Beryl and Lynn. Diseusses possible developments of additional irrigation supplies from surface and underground sources. Describes the quality of the water and contains field assays and laboratory analyses:

*219. Ground waters and irrigation enterprises in the foothill belt, southern California, by W. C. Mendenhall. 1908. 180 pp., 9 pls. $50 \mathrm{c}$.

Covers the Pasadena, Pomona, and Cucamonga quadrangles, which lie south of the San Gabriel Mountains between Los Angeles and San Bernardino. Describes the geography, geology, and ground-water conditions, discusses fluctuation in ground-water levels and the conservation of the water supply, and gives the results of measurements of water levels in 1904, 1905, and 1906. Describes irrigation systems and gives records of 1,044 wells. Contains maps showing original areas of artesian flow, areas of artesian flow in 1904, ground-water levels, irrigated lands, and locations of wells and pumping plants. Contains also the general map that is described under Water-Supply Paper 142 and is included with Water-Supply Papers 137, 138, and 139. 
*220. Geology and water resources of a portion of south-central Oregon, by G. A. Waring. 1908. $86 . \mathrm{pp}$., 10 pls. 20c.

Describes the geography and geology, and the streams, lakes, and ground waters in the valleys of Goose Lake, Abert Lake, Chewaucan Marsh, Summer Lake, Silver Lake, Christmas Lake, Alkali Lake, and in Warner Valley and several smaller valleys lying chiefly in Lake County. Gives analyses of waters and of alkali in soil and includes a geologic map.

221. Geology and water resources of the Great Falls region, Mont., by C. A. Fisher. 1909. 89 pp., 7 pls. 20c.

Describes the geography and geology, and the surface waters, ground,waters, and artesian conditions in an irregular area comprising parts of Cascade, Teton/ Fergus, Chouteau, and Lewis and Clark counties. Discusses municipal water supplies, the chemical character of the water, water powers, irrigation, and agriculture; gives analyses of water; includes maps showing the geology, the locations of artesian wells, etc.

*222. Preliminary report on the ground waters of San Joaquin Valley, Cal., by W. C. Mendenhall. 1908. 52 pp., 1 pl. $10 \mathrm{c}$.

Describes the geography, geology, and surface and ground waters of the entire valley. Discusses the origin, circulation, quantity, accessibility, and development of the ground waters; gives notes on the water supplies by counties; and includes a map showing areas of artesian flow and contours of the water table. Superseded by Water-Supply Paper 398.

*223. Underground waters of southern Maine, by F. G. Clapp, with records of deep wells, by W. S. Bayley. 1909. 268 pp., 24 pls. 55 c.

Covers an area that lies almost entirely south of the 45 th parallel. Describes the physiography, drainage, water-bearing rocks, the quantity, source, disposition, and temperature of the ground waters, and the recovery of water from springs, collecting galleries, tunnels, and wells. Discusses well-drilling methods and costs, municipal water supplies, and the quality of the ground waters. Gives detailed data for each county and records of deep wells. Includes a geologic map of southern Maine, and several diagrams showing the relative composition of waters from different kinds of rock.

*224. Some desert watering places in southeastern California and southwestern Nevada, by W. C. Mendenhall. 1909. 98 pp., 4 pls. $20 \mathrm{c}$.

Describes the physical features of the region, gives hints on desert traveling and on finding water in desert places, describes main routes of travel, and gives detailed descriptions of springs, wells, and other watering places. Includes a map of the region showing roads and watering places.

*225. Ground waters of the Indio region, Cal., with a sketch of the Colorado Desert, by W. C. Mendenhall. 1909. 56 pp., 12 pls. 20c.

Describes the geography, geology, precipitation, and drainage, and the source, character, and development of ground waters in the Colorado Desert, with special reference to the Indio region, which is the name applied to the artesian basin extending from the vicinity of Indio to the Salton Sea. Contains a table of well data and a map of the Indio region showing areas of artesian flow, irrigated lands, and locations of wells and pumping plants.

*227. Geology and underground waters of South Dakota, by N. H. Darton. 1909. 156 pp., 15 pls. $40 \mathrm{c}$.

Describes the geology and water horizons of the State, and discusses by counties the deep wells and well prospects. Gives notes on the construction and management of artesian wells. Includes maps showing the geology and the artesian conditions.

230, (See p.49.)

*231. Geology and water resources of the Harney Basin region, Oreg., by G. A. Waring. 1909. 93 pp., 5 pls. 25c.

Describes the geography, gelogy, surface waters, ground waters, and artesian conditions of the Harney, Catlow, Alvord, Whitehorse, and Malheur River basins, which lie chiefly in Harney County. Discusses the conservation of the water supply, the temperatures of ground waters, and well drilling methods and costs. Includes a geologio map of the area. 
232. Underground water resources of Connecticut, by H. E. Gregory, with a study of the occurrence of water in crystalline rocks, by E. E. Ellis. 1909.200 pp., 5 pls. $20 \mathrm{c}$.

Describes the physiography and geology, and the circulation, quantity, temperature, quality, and contamination of the ground water of the State. Discusses the water in the crystalline rocks, the Triassic sandstones and traps, and the glacial drift. Discusses also the methods of constructing wells and the character and use of the springs. Gives records of wells and springs and analyses of ground waters. Includes detailed descriptions of the towns of Warren, North Haven, and Branford Point.

233. Water resources of the Blue Grass region, Ky., by G. C. Matson, with a chapter on the quallty of the waters, by Chase Palmer. 1909. 223 pp., 3 pls. $20 \mathrm{c}$.

Describes the physiography, geology, soils, and water resources of an area covering 30 counties in the north-central part of Kentucky. Discusses the source, occurrence, quantity, and recovery of ground water, the artesian conditions, the collection and storage of rain water, and the municipal water supplies, the industrial uses and comparative hardness of the ground waters, and the various medicinal and table waters. Contains detailed data in regard to each county, and numerous well records and water analyses. Includes a geologic map of the area.

*234. Papers on the conservation of water resources. $1909.96 \mathrm{pp} ., 2 \mathrm{pls} .15 \mathrm{c}$.

Contains a paper on underground waters, by W. C. Mendenhall.

236. The quality of surface waters in the United States, Part I, Analyses of waters east of the one hundredth meridian, by R. B. Dole. 1909.123 pp. 10c.

Contains analyses of surface waters exclusively, but includes a description of analytical methods, an outline of methods for expressing analytical results, and a discussion of the probable accuracy of analyses that are applicable to ground waters as well as to surface waters. Describes methods for the following determinations: Turbidity, total suspended solids, total dissolved solids, silica, iron, calcium, magnesium, sodium and potassium, carbonates, bicarbonates, sul. phates, chlorine, nitrates, total acidity, and total iron. Also gives directions for the preparation of the necessary solutions.

240. Geology and water resources of the San Luis Valley, Colo., by C. E. Siebenthal. 1910. 128 pp., 13 pls. 25c.

Describes the geography and geology, and the artesian and other waters of the valley. Gives detailed data regarding the springs and the flowing and nonflowing wells, and discusses adequacy and permanence of the artesian supply, variations in flow, and temperature, quality and uses of the water, well-drilling methods and costs, and approximate methods for measuring the discharge of flowing wells. Contains several analyses and a map showing the area of artesian flow, the gas fields, and the area of colored water.

247. (See p. 49.)

250. (See p. 49.)

251. Surface-water supply of the United States, 1907-8, Part XI, California. 1910. 363 pp., 7 pls. 35 c.

Contains a section (pp. 338-348) on "fluctuations in ground-water levels in the valley of southern California, by W. C. Mendenhall, in which are given results of measurements of depths to the water level in typical wells during 1907-8. These measurements were a continuation of the work reported in Water-Supply Paper 213. Contains also records of the discharge of springs as follows: Fish Springs, Seeley Springs, and Black Rock Springs, Cal. (p. 333); Bettles Rest Springs, Oreg. (p. 337); Fords Springs, and Olene Springs, Cal. (p. 338).

252. (See p. 49.)

254. The underground waters of north-central Indiana, by S. R. Capps, with a chapter on the chemical character of the waters, by R. B. Dole. 1910. 279 pp., 7 pls. 40c.

Covers the following 19 counties: Boone, Carroll, Cass, Clinton, Elkhart, Fulton, Grant, Hamilton, Hancock, Hendricks, Howard, Kosciusko, Madison, Marion, Marshall, Miami, St. Joseph, Tipton, and Wabash. Describes the geography and geology, the sources, movements, occurrence, and quantity of ground water and the methods of constructing wells and of lifting water. Describes in detail, for each county, the ground-water conditions and the water supplies for cities, villages, and rural districts. Discusses the methods of making water analyses and of expressing the results, the mineral constituents of natural waters, the influence of these constituents upon domestic, industrial, and medicinal uses of the water, and methods of purification. Compares the chemical composition of the waters in different geologic formations in the area and gives numerous analyses and fleld assays. Contains maps showing the distribution of rock formations and surface deposits, the thickness of the surface deposits, and the areas of artesian flow. 
*255. Underground waters for farm use, by M. L. Fuller. 1910.' 58 pp., 17 pls. $15 \mathrm{c}$. Discusses the various kinds of water-bearing formations and the relative safety of supplies from each. Describes different types of springs and their protection from pollution. Discusses dug and drilled wells with respect to their location, yield, cost, and safety from pollution. Discusses also cisterns and combination wells and cisterns.

256. Geology and underground waters of southern Minnesota, by C. W. Hall, O. E. Meinzer, and M. L. Fdller. 1911. 406 pp., 18 pls. $60 \mathrm{c}$.

Covers Bigstone, Swift, Kandiyohi, Meeker, Wright, Anoka, and Washington counties and, all of Minnesota south of these counties. Discusses the physiography, geologic history, geologic formations and their water-bearing capacities, artesian conditions, mineral quality of the ground waters, types of wells, methods of finishing wells in sand, methods of drilling in quartzite, "blowing" and "breathing" of wells, freezing of wells and other phenomena due to variations in atmospheric pressure, drainage into wells, and municipal water supplies. Gives detailed data, by counties, concerning the yield, head, and quality of water. Includes numerous analyses of water and maps showing the thickness and character of surface deposits, the depths to granitic rocks and Sioux quartzite, the distribution of water-bearing formations, the areas of artesian flow, and the quality of ground waters; also diagrams showing geographic variations in the quality of the waters from surface deposits and bedrocks.

*257. Well-drilling methods, by Isaiah Bowman. 1911.139 pp., 4 pls. $15 \mathrm{c}$.

Discusses briefly ground water in the United States and water-bearing formations; gives a history of well drilling in Asia, Europe, and the United States; and describes the various methods of drilling and the machinery used. Discusses the difficulties encountered in sinking wells, the flooding of oil wells, the contamination of water wells and methods of preventing contamination, the capacity of wells and methods of testing capacity, methods of measuring the depth of wells and of detecting the defection of drill holes, and the cost of sinking wells.

*258. Underground-water papers, 1910, by M, L. Fuller, F. G. Clapp, G. C. Matson, Samuel Sanford, and H. C. Wolff. 1911. 123 pp., 2 pls. $15 \mathrm{c}$.

Contains the following papers:

Drainage by wells, by M. L. Fuller (pp. 6-22).

Freezing of wells and related phenomena, by M. L. Fuller (pp. 23-31).

Occurrence and composition of well waters in the slates of Maine, by F. G. Clapp (pp. 32-39).

Occurrence and composition of well waters in the granites of New England, by F. G. Clapp. (pp. 40-47).

Pollution of underground waters in limestone, by G. C. Matson (pp. 48-56).

Protection of shallow wells in sandy deposits, by M. L. Fuller (pp. 57-65).

Composition of mineral springs in Maine, by F. G. Clapp (pp. 66-74).

Saline artesian waters of the Atlantic Coastal Plain, by Samusl Sanford (pp. 75-86).

Magnetic wells, by M. L. Fuller (pp. 87-93).

Underground waters near Manassas, Va., by F. G. Clapp (pp. 94-97).

The utilization of the underflow near St. Francis, Kans., by H. C. Wolff (pp. 98-119).

259. The underground waters of southwestern Ohio, by M. I. Fuller and F. G. Clapp, with a discussion of the chemical character of the waters, by R. B. Dole. 1912. 228 pp., 9 pls. 35c.

Covers the following counties: Adams (western half), Brown, Butler, Clark, Clermont, Clinton, Darke (southern part), Greene, Hamilton, Highland (western half), Miami (southern part), Montgomery, Preble, and Warren. Describes the topography and geology, the waterbearing formations, the source, occurrence, and head of the water, and the municipal water supplies. Givesdetailedinformation inregard toground-water conditions by counties. Discusses methods of making water analyses and of expressing the results, mineral constituents of natural water and their effects with respect to its use for domestic, industrial, and medicinal purposes, and methods of púrifying water. Gives numerous analyses and field assays. Includes maps showing the rock formations, the surface deposits, the thickness of surface deposits, structure contours, and areas of artesian flow. The chapter on the chemical character of the water is nearly the same as the chapter on the same subject in Water-Supply Paper 254.

*260. Preliminary report on the ground waters of Estancia Valley, N. Mex., by O. E. Meinzer. 1910 . $33 \mathrm{pp}$. $5 \mathrm{c}$.

Describes briefly the ground-water conditions in the valley and discusses the use of ground water for irrigation. Includes analyses but no maps. This report is superseded by WaterSupply Paper 275.

271. (See p. 49.) 
273. Quality of the water supplies of Kansas, by H. N. Parker, with'a preliminary report on stream pollution by mine waters in southeastern Kansas, by E. H.S. Bailey. 1911. 375 pp., 1. pl. 30c.

Describes the geology, ground water, and artesian basins of the State; discusses the significance of mineral constituents and classification of water; gives details concerning quality of ground water by counties and surface water by drainage basins; contains numerous assays and analyses of surface and ground waters; includes a geologic map of the State.

274. Some stream waters of the western United States, with chapters on sediment carried by the Rio Grande and industrial application of water analyses, by Herman Stabler. 1911. 188 pp. 15c.

Contains analyses of surface waters exclusively, but the discussion of industrial application of water analyses which it includes is as pertinent for ground waters as for surface waters. This discussion introduces "reacting coefficients" and "reacting values" and develops formulae for calculating, from analysis, the soap-consuming power of the water, the amount of softening constituents that it requires, the extent of foaming, priming, and corrosion that it will produce in boilers, the amount of scale and the hardness of the scale that it will deposit in boilers, and its quality for irrigation.

275. Geology and water resources of Estancia Valley, N. Mex., with notes on groundwater conditions in adjacent parts of central New Mexico, by O. E. Meinzer. 1911. 89 pp., 14 pls. $20 \mathrm{c}$.

Describes the physiography, geology, soil, and climate of the valley, and discusses the source and disposal of ground water, the water table, artesian conditions, yields of wells, quantity of ground water available, quality of ground water, storage of storm waters, use of ground water for irrigation, types of wells, windmills, cost of pumping, and the alkali problems. Contains tables giving depths to water level in wells and analyses and assays of water from wells and springs. Contains also brief reports on physiography, geology, soil, ground water, and irrigation in Encino and Pinos Wells basins, and notes on wells at Vaughan. Includes maps showing physiography and Pleistocene and Recent geology, depths to the water table, and amount of chlorides and sulphates found in the ground waters.

*276. Geology and underground waters of northeastern Texas, by C. H. Gordon. 1911. 78 pp., 2 pls. $10 \mathrm{c}$.

Covers an area comprising Bowie, Camp, Cass, Delta, Franklin, Hopkins, Lama, Morris, Red River, and Titus counties. Describes the geography and geology, and the artesian and other waters found in the various formations. Describes the water resources by counties. Gives tables of well data and analyses of ground waters and includes a geologic map of the area.

277. Ground water in Juab, Millard, and Iron counties, Utah, by O. E. Meinzèr. 1911. $162 \mathrm{pp} ., 5 \mathrm{pls}$. $25 \mathrm{c}$.

Describes briefly the physiography, geology, precipitation, soil, vegetation, streams, and industrial development, occurrence of water in bed rock and in unconsolidated sediments, artesian conditions, springs, the quality of ground waters, irrigation, construction of wells, and watering places on routes of travel. Describes in more detail Juab, Round, Little, Sage, Dog, Fernow, and Tintic valleys, the Tintic mining district, Pavant and Lower Beaver valleys, Old River Bed, Cherry Creek, the Drum, and Swasey Wash regions, Sevier Desert, Wah Wah Valley, Sevier Lake bottoms, White, Fish Springs, Snake, Parowan, and Rush Lake valleys and the Escalante Desert. Contains several water analyses.

278. Water resources of Antelope Valley, Cal., by H. R. Johnson. 1911. 92 pp., 7 pls. 25c.

Describes the drainage, climate, and physiography, and the water-bearing and non waterbearing formations of an area in Kern, Los Angeles, and San Bernardino counties. Discusses the artesian and other ground waters, the chemical character of the ground waters, certain fallacies as to the origin and quantity of artesian water, and the present and future development of the underground supplies. Contains a table of well data and a few chemical analyses, and includes a map showing water-bearing and nonwater-bearing formations and the area of artesian flow.

288. (See p. 49.)

289. (See p. 49.) 
293. Underground-water resources of Iowa, by W. H. Norton, W. S. Hendrixson, H. E. Simpson, O. E. Meinzer, and others. 1912. 994 pp., 18 pls. , 70c.

Describes the topography, climate, and geology of the State, the occurrence of water in the various geologic formations, the artesian phenomena and the yields of artesian wells, the chemical composition of the ground waters, the municipal, domestic and industrial water supplies, and methods of drilling wells. Discusses corrosion of well casings and boilers and the deposition of scale in boilers. Gives a classification of mineral waters. Contains numerous sections of wells and about 400 water analyses. Gives detailed information concerning ground waters and city and village supplies by districts and counties. Includes maps showing the glacial and rock geology, structure contours of water-bearing formations, locations of deep wells, head of artesian water, and quality of ground water. Also includes numerous geologic sections showing dépths to the principal water-beaning formations. Describes a method of casing deep wells with cement (p. 562).

294. An intensive study of the water resources of a part of Owens Valley, Cal., by C. H. Lee. 1912.135 pp., 30 pls. $55 \mathrm{c}$.

Relates to the Independence region, a segment of Owens Valley that is relatively isolated with respect to water supplies. Describes the underground reservoir of this region and the drainage basin tributary to it. Presents quantitative data on precipitation, stream flow, percolation into the underground reservoir from precipitation, streams, irrigation, and flood waters, evaporation and transpiration from soils in experimental tanks with various depths to water level, fluctuations of the water table, height of capillary rise of ground water, areas with specifled depths to the water table within the range of capillary rise, and discharge from springs. Analyzes the data and calculates the annual intake and discharge of the underground reservoir and the available supply of ground water. Includes maps showing depths to ground water. and other bydrologic features and also includes numerous diagrams. The results of the tank experiments and their application in estimating the discharge of ground water in closed desert basins are of general interest.

298. (See p. 49.)

*300. Water resources of California, Part III, Stream measurements in the Great Basin and Pacific coast river basins, by H. D. McGlashan and H. J. Dean. 1913. 956 pp., 4 pls. $55 \mathrm{c}$.

Gives discharge records of springs and wells in California as follows: Grover Hot springs (p. 198), Black Rock Springs (p. 393), Seeley Springs (p. 394), Slanson well (p. 680), Shasta Little Springs (p. 913), Anna Creek Spring (p. 914), Bettles Rest Springs (p. 915), Barclay Springs (p. 915), Fords Spring (p. 917), and Olene Springs (p. 918).

310. (See p. 49.)

314. (See p. 49.)

*315. The purification of public water supplies, by G. A. Johnson. 1913. $84 \mathrm{pp}$. 8 pls. 10c.

Includes a brief discussion of ground waters for municipal supplies; also information on methods of purification that are more or less applicable to ground waters.

316. Geology and water resiources of a portion of south-central Washington, by G. A. Waring. 1913. $46 \mathrm{pp} ., 1 \mathrm{pl}$. $5 \mathrm{c}$.

Covers an area of about 5,000 square miles comprising Benton County and parts of Franklim, Grant, Yakima, and Klickitat counties. Describes the climate, vegetation, physiography, and geology; discusses shallow and artesian waters and irrigation enterprises in Sumyside and Reservation valleys, Horse Heaven Plateau, and the Columbia River plains, and irrigation along lower Yakima River; gives tabulated data concerning wells and springs; includes a geologic map.

*317. Geology and underground waters of the Wichita region, north-central Texas, by C. H. Gordon. 1913. 88 pp., 2 pls. 10c.

Covers Archer, Baylor, Clay, Foard, Hardeman, Haskell, Jack, Knox, Montague, Throckmorton, Wichita, Wilbarger, and Young counties. Describes the physiography and geology, the occurrence and quality of ground waters and their relation to rock structure, the effects of barometric changes on water levels, and the water-bearing formations; gives detailed information by counties; contains numerous sections of wells and water analyses; includes a geologic map. 
318. Water resources of Hawaii, 1909-1911, by W. F. Martin and C. H. Pierce. 1913. 552 pp., 15 pls. $50 \mathrm{c}$.

Describes chiefly surface-water supplies, but contains brief notes on ground-water supplies and data on the discharge of springs, infiltration tunnels, flowing wells, and pumped wells on several islands, as follows: Kauai, pumped wells (p. 144); Oahu, springs, pumped wells, and flowing wells (pp. 163, 187-196); Maui, pumped wells (pp. 258, 331-332); Hawaì, springs and infiltration tunnels (p. 408). Gives sections of wells on Oahu (pp. 191-193).

*319. Geology and ground waters of Florida, by G. C. Matson and Samuel Sanford. 1913. 445 pp., 17 pls. $60 \mathrm{c}$.

Describes the characteristic upland, lowland, and coastal features of the State--the springs, lakes, caverns, sink holes, natural bridges, torraces, sand dunes, coral reefs, bars, inlets, tidal runways, pine lands, swamps, keys, and ocean currents. Describes in detail the geologic formations, the source, quantity, depth, circulation, and recovery of artesian and other ground waters. Gives detailed information concerning ground-water conditions and water supplies by counties. Contains numerous well sections and tables of well data and includes maps showing the geology and the Pleistocene terraces of the State.

320. Geology and water resources of Sulphur Spring Valley, Ariz., by O. E. Meinzer and F. C. Kelton, with a section on agriculture, by R. H. Forbes. 1912. 231 pp., 15 pls. $45 \mathrm{c}$.

Covers Sulphur Spring Valley and contains a small amount of information on San Pedro, San Simon, and San Bernardino valleys. Describes the physiography, the drainage, the geology, with special reference to the Quaternary deposits in the valley, the seasonal and geographic distribution of the precipitation, the occurrence and level of the ground water, the flowing and nonflowing wells, the quality of ground waters with relatfon to derivative rocks, water levels, and underground eirculation, the effects of quality on irrigation and other uses, the distribution of alkali in the soil, the relation of the alkali to the water table and to the drainage, and the relation of zones of vegetation to water supply and other geographic controls. Contains detailed data in regard to tests of 20 pumping plants and describes a portable weir used in making these tests. Gives the history of agriculture in the blley and discusses agricultural meth ods. Contains analyses of water and of alkali in the soil and includes maps showing the geology, vegetation, depths to ground water, elevation of the water table, quality of water, and alkali in soil.

326. (See p. 49.)

331. Surface water supply of the United States, 1912, Part X, Pacific coast basins - in California, by H. D. McGlashan and G. C. Stevens. 1914. 442 pp., 2 pls. 30c.

Contains records for 1909 to 1912, inclusive, of water levels in the series of wells for which water-level data are given in Water-Supply Papers 213 and 251, with an introductory note by W. C. Mendenhall (pp. 425-434).

332. (See p. 49.)

333. Ground water in Boxelder and Tooele counties, Utah, by Everett Carpenter. 1913. 90 pp., 2 pls. 10c.

Covers all of Boxelder County and Tooele, Rush, and skull valleys in Tooele County. Describes briefly the geography, geology, water in bedrock and in unconsolidated sediments, artesian conditions, springs, and quality of ground waters; gives detailed information by valleys; contains numerous assays of water; includes a guide to watering places on routes of travel and maps showing locations of flowing and nonflowing wells, springs, and roads.

*335. Geology and underground waters of the southeastern part of the Texas Coastal Plain, by Alexander Deussen. 1914.365 pp., 9 pls. $55 \mathrm{c}$.

Covers that part of the Coastal Plain of Texas occupied by the outcrop of Cenozoic rocks east of Brazos River and south of a line extending east and west through Jefferson, in Marion County. Describes the physiography, stratigraphy, geologic structure, occurrence of ground water, springs, artesian systems, and quality of water with reference to various uses and in relation to the geologic formations; gives detailed information by counties; contains numerous well sections and water analyses; includes maps showing the geology of the region and the structure contours and areas of artesian flow for the seven principal artesian reservoirs. 
336. Water resources of Hawaii, 1912, by C. H. Pierce and G. K. Larrison. 1914. $392 \mathrm{pp} .50 \mathrm{c}$.

Relates chiefly to surface waters, but contains data in regard to pumpage of wells and seepage of ditches on the Island oi Kauai (p. 99), discharge of springs and flowing wells and fluctuations of water levels in wells on the Island of Oahu (p. 128), pumpage of wells on the Island of Maui (pp. 209-211), and of springs on the Island of Hawaii (p. 381).

338. Springs of California, by G. A. Waring. 1915.410 pp., 13 pls. $60 \mathrm{c}$.

Describes the physical features of California and discusses natural waters with respect to mineral analyses, source and amount of dissolved substances, therapeutic and other properties, temperature, and classiffeation. Gives detailed descriptions of springs, particularly those which yield mineral waters or are used as centers of recreation or health resort. Contains numerous analyses and includes maps showing the geology of the State and the locations of hot, carbonated, and sulphur springs.

*340. Stream-gaging stations and publications relating to water resources, 1885-1913, by B. D. Wood. $1916.195 \mathrm{pp} .15 \mathrm{c}$.

Includes papers dealing with ground water. Issued also in separate chapters as follows:

*(a) Part I. North Atlantic coast drainage basins, pp. 1-19.

(b) Part II. South Atlantic coast and eastern Gulf of Mexico drainage basins, pp. 21-30.

(c) Part III. Ohio River basin, pp. 31-42.

(d) Part IV. St. Lawrence River basin, pp. 43-52.

(e) Part V. Hudson Bay and upper Mississippi River drainage basins, pp. 53-61.

(f) Part VI. Missouri River basin, pp. 63-81.

(g) Part VII. Lower Mississippi River basin, pp. 83-93.

(h) Part VIII. Western Gulf of Mexico drainage basins, pp. 95-104.

(i) Part IX. Colorado River basin, pp. 105-116.

(j) Part X. The Great Basin, pp. 117-129.

*(k) Part XI. Pacific coast basins in California, pp. 131-146.

*(l) Part XII. North Pacific slope drainage basins, pp. 147-195.

341. Underground waters of the Coastal Plain of Georgia, by L. W. Stephenson and J. O. Veatch, and a discussion of the quality of the waters, by R. B. Dole. 1915. 539 pp., 21 pls. 50c.

Describes the physiography and geology, the source, quantity, and disposition of water supplies, the artesian and nonartesian waters, the quality and use of water from springs and wells, and the stratigraphic distribution of the ground water. Gives detailed information by counties. Discusses the quality of the water in relation to industrial, domestic, and medicinal uses, the puriffeation of water, and the composition of surface waters, and of ground waters in relation to geologic formations, geographic position, and depth. Contains many well sections and water analyses and includes maps showing the geology, the areas of artesian flow and the locations and depths of wells.

343. Geology and water resources of Tularosa Basin, N. Mex., by O. E. Meinzer and R. F. Hare. 1915.317 pp., 19 pls. $40 \mathrm{c}$.

Covers a large area in south-central New Mexico between the Rio Grande and Pecos valleys, and also contains data in regard to the vicinity of El Paso, Tex. Describes the physiography and geology and gives data on precipitation and its seasonal and geographic distributions. Discusses the water in the valley fill in regard to intake zones, occurrence, disposal, yield, artesian head, methods of construeting wells, and quality of the water in relation to derivative rocks, water table, and water-bearing beds and with respect to its use for drinking, cooking, washing, steam making, and irrigation. Discusses likewise the water in Cretaceous and Carboniferous strata and in igneous rocks. Describes the soil and native vegetation in relation to water supplies, the irrigation from streams, springs, flood waters, and wells, the railroad and public water supplies. Contains a guide to watering places on routes of travel, and tables of well data, analyses of water and soil, and distances between watering places. Includes maps showing the geology, vegetation, depths to water, contours of the water table, and locations of watering places and connecting roads.

*345. Contributions to the hydrology of the United States, 1914; N. C. Grover, chief hydraulic engineer. $1915.225 \mathrm{pp} ., 17 \mathrm{pls} .30 \mathrm{c}$.

Issued also in separate chapters. 'The following chapters relate to ground water:

(a) Preliminary report on ground water for irrigation in the vicinity of Wichita, Kans., by o. E. Meinzer (pp. 1-9).

(b) Ground water for irrigation in the vicinity of Enid, Okla., by A. T. Schwennesen (pp. 11-23, PI. I). Includes a note on ground water for irrigation on the Great Plains, by $O$. E. Meinzer. 
*345. Contributions to the hydrology of the United States, 1914-Continued.

(c) Underground water of Luna County, N. Mex., by N. H. Darton (pp. 25-40, Pl. II). Includes the results of flve pumping tests made by A. T. Schwennesen.

- (d) Ground water for irrigation in the valley of North Fork of Canadian River near Oklahoma City, Okla., by A. T. Schwennesen (pp. 41-51, Pl. III).

(g) The water resources of Butte, Mont., by O. E. Meinzer (pp. 79-125, Pls. VII-VIII).

(h) Ground- water resources of the Niles cone and adjacent areas, Cal., by W. O. Clark (pp. 127-168, PIs. IX-XVII).

All except No. 345-a include maps showing ground-water conditions. No. 345-h contains numerous records of water levels in wells and an estimate of ground-water recharge in 1912-13 and 1913-14 based on fluctuations of the water table. It includes a series of graphs showing fluctuations of the water table and the source of the ground water.

358. Water resources of the Rio Grande Basin, 1888-1913, by Robert Follansbee, H. J. Dean, W. W. Follett, and G. A. Gray, 1915.725 pp., 3 pls.

Contains data on seepage of water into and out of streams, and discussions of the data (pp. 679-694).

359. (See p. 49.)

360. (See p. 49.)

362. (See p. 49.)

364. Water analyses from the laboratory of the United States Geological Survey, tabulated by F. W. Clarke. 1914. 44 pp. 5c.

Contains 203 miscellaneous analyses of waters from'rivers, lakes, wells, springs, and mines, which were made at various times in the chemical laboratory of the United States Geological Survey. Includes analyses of waters from wells, springs, or mines in the following 25 States: Arkansas, Arizona, California, Colorado, Florida, Illinois, Iowa, Kentucky, Maine, Michigan, Mississippi, Missouri, Montana, Nevada, New Mexico, North Carolina, Oklahoma, Pennsylvania, South Carolina, Tennessee, Texas, Utah, Virginia, West Virginia, and Wyoming.

365. Ground water in southeastern Nevada, by Everett Carpenter. 1915. 86 pp., 5 pls. $15 \dot{c}$.

Covers an area of about 17,000 square miles in Clark, Lincoln, White, Pine, and Nye courities, including the vicinities of Geyser, Lund, Barnes, and Duckwater, on the north, and the vicinities of Sharp, Hiko, Alamo, and Indian Springs, on the west. Describes briefly the geography, geology, water in bedrock, and in unconsolidated deposits, artesian conditions, springs, and quality of water. Gives detailed information regarding water supplies in the Las Vegas drainage basin, Duck, Ursine, and Meadow valleys, White River drainage basin, Muddy and Virgin valleys, and Bristol, Delamar, Coal, Garden, Dry Lake, Indian Spring, and Railroad valleys. Contains water analyses, a guide to watering places on routes of travel, and a table of distances between watering places. Includes maps showing Pleistocene lake beds, areas of artesian flow, and locations of watering places.

370. (See p. $49=50$.)

373. Water resources of Hawaii, 1913, by G. K. Larrison. 1915.190 pp. $20 \mathrm{c}$.

Relates chiefly to surface waters, but contains data on the discharge of springs, tunnels, and flowing wells on the island of Oahu (p. 103), springs on the island. of Maui (p. 151), and springs on tho island of Hawaii (p. 174).

374. Ground water in the Hartford, Stamford, Salisbury, Willimantic, and Saybrook areas, Conn., by H. E. Gregory and A. J. Ellis. 1916. 150 pp., 13 pls. 30c.

Covers the towns of Bloomfield, Canaan, East Hartford, East Windsor, Essex, Franklin, Greenwich, Hartford, Manchester, Newington, North Canaan, Old Lyme, Salisbury, Saybrook, Stamford, South Windsor, Westbrook, West Hartford, Wethersfield, Windham, and Windsor. Discusses the origin, circulation, quantity, and quality of the waters in stratified and unstratified drift, crystalline rocks, traps, Paleozoic limestones, and Triassic sandstones. Discusses ground water for municipal use and the construction of drilled, driven, and dug wells, and infiltration galleries. Describes the municipal pumping plants at Brookline, Mass., Brooklyn, N. Y., and Plainfiold, N. J. Describes the ground water conditions in detail, by towns. Contains numerous tables of well data and water analyses, and includes maps showing water-bearing formation, depths to water, locations of wells, and woodlands. 
*375. Contributions to the hydrology of the United States, 1915; N. C. Grover, chief hydraulic engineer. 1916.181 pp., 9 pls. $15 \mathrm{c}$.

Issued also in separate chapters. The following chapters relate to ground water:

(a) Ground water for irrigation in the Sacramento Valley, Cal., by Kirk Bryan (pp. 1-49. Pls. I-II).

(b) Ground water in Paradise Valley, Ariz., by O. E. Meinzer and A. J. Ellis (pp. 51-75, Pls. III-V).

(d) Ground water in Big Smoky Valley, Nev., by O. E. Meinzer (pp. 85-116, Pls. VI-VII).

(g) Ground water in Lasalle and McMulien counties, Tex., by Alexander Deussen and R.B. Dole (pp. 141-177, Pls. VIII-IX).

All of these papers include maps showing ground-water conditions. No. 375-A contains statistics on irrigation with ground water in Sacramento Valley and a discussion of problems relating to the construction of wells and to pumping for irrigation. No. 375-D contains data on groundwater intake and discharge and on irrigation with ground water. No.375-G discusses water in the various geologic formations, especially with reference to its quality and quiantity for irrigation; it contains tables of well data and water analyses.

380. The Navajo country-a geographic and hydrographic reconnaissance of parts of Arizona, New Mexico, and Utah, by H. E. Gregory. 1916. 219 pp., 29 pls. $80 \mathrm{c}$.

Covers the Navajo and Hopi Indian reservations, in northeastern Arizona, northwestern New Mexico, and southeastern Utah, and some adjacent areas. Gives an outline of the history of the region; describes the geographic provinces, climate, soil, flora, fauna, and geology; deseribes also the streams and discusses factors influencing stream flow, irrigation with surface waters, storage of rain and surface waters for domestic and stock use, and water powers; discusses groundwater reservoirs, quality of ground water, artesian water, springs, and wells; gives data on watering places and recommendations for prospecting for water in various geographic provinces. Includes maps showing the geology, the locations of watering places, roads, and trails, and the areas covered by forests.

389. (See p. 50.)

390. (See p. 50.)

393. (See p. 50.)

394. (See p. 50.)

*397. Ground water in the Waterbury area, Conn., by A. J. Ellis, under the direction of H. E. Gregory. 1916.73 pp., 4 pls. $15 \mathrm{c}$.

Discusses the water in glacial drift and crystalline rocks, ground water for privateand municipaI uses, and methods of developing ground-water supplies. Describes the municipal pumping plants at Brookline, Mass., Brooklyn, N. Y., and Plainfield, N.J. Describes in detail the waterbearing formations and water supplies in the towns of Ansonia, Beacon Falls, Middlebury, Naugatuck, Oxford, Seymour, Thomaston, Waterbury, and Watertown. Contains tables of well data and water analyses, and includes a map showing areas underlain by stratiffed drift, rock outcrops, woodlands, and locations of wells and springs.

398. Ground water in San Joaquin Valley, Cal., by W. C. Mendenhall, R. B. Dole, and Herman Stabler. 1916. 310 pp., 5 pls. $25 \mathrm{c}$.

Describes the development of irrigation in the Southwest and gives an outline of the geography and geology of the San Joaquin basin. Discusses briefly the origin, eirculation, quantity, and availability of ground water, and its use for irrigation. Describes the quality of the surface and ground waters, the standards for classification, the methods of purifying water, and the effects of quality on use. Explains the variations in the quality of the water with its depth below the surface and with its geographic and geologic relations. Gives details of 55 pumping tests and summarizes and discusses the results. Gives numerous well records'and water analyses and detailed descriptions by counties. Includes maps showing areas of artesian flow, contours of the water table, quality of ground water, and locations of the pumping plants investigated. 
399. Geology and ground waters of northeastern Arkansas, by L. W. Stephenson and A. F. Crider, with a discussion of the chemical character of the waters, by R. B. Dole. 1916. 315 pp., 11 pls. 35c.

Covers an area of about 13,250 square miles extending from Mississippi River west to the Ozark province and from Missouri south to Arkansas River. Describes the physiography, geology, surface waters, stratigraphic distribution of ground waters, springs, artesian waters, and uses of ground water, especially in irrigating rice. Gives detailed descriptions, well sections, and tables of well data by counties. Discusses chemical standards of classification, methods of purification, and the quality of the waters in relation to the strata in which they occur, their geographic positions, and their depths below the surface. Includes numerous analyses and maps showing the geology, the areas of artesian flow, and the locations and depths of wells.

*400. Contributions to the hydrology of the United States, 1916; N. C. Grover, chief hydraulic engineer. 1917. 108 pp., 7 pls.

Issued also in separate chapters. The following chapters relate to ground water:

(b) Artesian water for irrigation in Little Bitterroot Valley, Mont., by O. E. Meinzer (pp. 9-37, Pls. I-IV).

(e) Ground water for irrigation in the Morgan Hill area, Cal., by W. O. Clark (pp. 61-106, Pls. V-VII).

Both papers include maps showing ground-water conditions. No. 400-E contains numerous well sections and records of water levels in wells and an estimate of the annual ground-water supply, based chiefly on fluctuations of the water table and porosity of the water-bearing deposits.

416. The divining rod, a history of water witching, with a bibliography, by A. J. Ellis. 1917. $55 \mathrm{pp}$.

Gives an outline of the history of the popular delusion known as "water witching" and points out fallacies in so-called "mechanical water finders." Advises the public against expending money "for the services of any water witch or for the use or purchase of any machine or instrument devised for locating underground water or other minerals." List 3 numerous papers on the subject from 1532 to the present time.

418. Mineral springs of Alaska, by G. A. Waring, with a chapter on the chemical character of some surface waters of Alaska, by R. B. Dole and A. A. Chambers 1917. 114 pp., 9 pls.

Describes hot springs in 48 localities, carbonated springs in 12 localities, and sulphur springs in 13 localities, also iron springs and salt springs. Discusses the chemical character of the waters of Yukon, Tanana, Lowe, Copper, Stikine, and other rivers. Contains 32 analyses of spring waters and 38 analyses of surface waters. Includes a map of Alaska showing the locations of 108 groups of mineral springs in relation to volcanoes.

422. Ground water in Animas, Playas, Hachita, and San Luis basins, N. Mex., by A. T. Schwennesen (in press).

Covers the southern part of Grant County. Describes the physiography and geology and the ground-water conditions in each basin with respect to the occurrence, depth, quantity, quality, artesian conditions, and irrigation prospects. Gives well data, analyses of water, and analyses of the water-soluble contents of the soil. Contains a map of the area showing depths to the water table and other features.

423. Geology and water resources of Big Smoky, Clayton, and Alkali Spring valleys, Nev., by O. E. Meinzer. 1917. 167 pp., 15 pls.

Describes the physiography and geology with special reference to Quaternary events; gives data on precipitation, stream flow, seepage, springs, and wells; estimates intake and discharge of ground water; discusses criteria for recognizing shallow-water areas; discusses the quality of the water with reference to the geologic source of the valley fill, geographic provinces, and use describes public water supplies and discusses irrigation with ground water; contains analyses of water and of alkali in soil; includes maps showing Pleistocene iake features, depths to ground water, areas of ground water intake and discharge, and locations of watering places. 
425. Contributions to the hydrology of the United States, 1917 ; N. C. Grover, chief hydraulic engineer. 1918.

Issued also in separate chapters. The following papers relate to ground water:

(a) Ground water in San Simon Valley, Ariz., by A. T. Schwennesen, with a chapter on agriculture by R. H. Forbes (pp. 1-35, Pls. I-III). Describes the physiography and geology of the valley, the upper water horizon, and the deeper artesian horizon of the San Simon and Bowie areas, the ground water in the Rodeo and Artesia valleys, and theirrigation suppliesfrom flowing and nonflowing wells; contains 39 analyses of welland spring waters, numerous records of deep wells and maps showing areas of artesian flow, depth to water table, and lands irrigated with well water; also includes a chapter by R. H. Forbes on soil, vegetation, and agricultural prospects.

(b) Ground water forirrigation in Lodgepole Valley, Wyo.-Nebr., by O. E. Meinzer (pp.37-69 Pls. TV-VI). Describes the physiography and geology of Lodgepole Valley and the adjacent region and the water in the alluvial gravel and in the Tertiary and Cretaceous formations; discusses irrigation with ground water; gives well data and analyses of 20 well waters and 2 samples from Lodgepole Creek; contains maps showing the geology and the depths to the water table; also includes data on the cost of pumping for irrigation in western Nebraska, by H. C. Diesem, U. S: Department of Agriculture.

(d) Ground water in Reese River valley and adjacent parts of Humboldt River valley, $\mathrm{Nev}_{\text {, }}$ by G. A. Waring (pp. 95-129, Pls., VII-XII). Describes the physiography, geology, groundwater conditions, and irrigation prospects. Contains well and spring data and analyses of ground waters. Includes a map showing the geology, shallow-water areas, and areas of artesian flow.

(e) Ground water in Quincy Valley, Wash., by A. T. Schwennesen and O. E. Meinzer (pp. 131-158, Pl. XIII-XIV). A preliminary report which outlines the physiographic features, climate, and agrieultural conditions of Quincy Valley and adjacent regions; describes the character and distribution of the Yakma basalt, Pleistocene lake beds, and Pleistocene outwash gravels, and discusses quantity, quality, and head of water in each of these formations; discusses present and prospective irrigation with ground water. Contains a sketch map showing contours of the water table.

428. Artesian water in the vicinity of the Black Hills, S. Dak., by N. H. Darton (in press).

Describes the geology and artesian-water conditions in areas covered in previous reports but in the light of additional data. Discusses the artesian prospects of the Dakota, Minnelusa, and Deadwood sandstones. Contains a map showing the geology and the depths to the waterbearing sandstones.

430. Surface water supply of Hawaii, July 1, 1913, to June 30, 1915; N. C. Grover, chief hydraulic engineer; G. K. Larrison, district engineer. 1917.329 pp.

Contains data on the discharge of springs, tunnels, and flowing wells on the Island of Oahu (pp. 205-207).

445. Surface water supply of Hawaii, July 1, 1915, to June 30, 1916; N. C. Grover, chief hydraulic engineer; G. K. Larrison, district engineer. 1917. 224 pp.

Contains discharge records for springs near Kailua, Oahu (pp. 97-99), and for Kahoma devejopment tunnel, near Lahaina, Maui (pp. 156, 157).

\section{ANNUAL REPORTS.}

*Fifth Annual Report (1883-84). 1885.469 pp., 58 pls. $\$ 2.25$.

The following paper relates to ground water:

*(A $5 \mathrm{c}$ ) The requisite and qualifying conditions of artesian wells, by T. C. Chamberlin (pp. 125 to 173, Pl. XXI). This is the first paper published by the United States Geological Survey on the subject of ground water. It is a clear, accurate, and comprehensive statement of the conditions that produce artesian basins and give rise to flowing wells, and is regarded as authoritative on the subject.

*Ninth Annual Report (1887-88). 1889.717 pp., 88 pls. $\$ 2$.

The following paper relates to ground water:

*(A 9 d) Formation of travertine and siliceous sinter by the vegetation of hot springs, by W. H. Weed (pp. 613-676, Pls. LXXVIII-LXXXVII). Describes the Mammoth Hot Springs and other hot springs and geysers of Yellowstone National Park, also hot springs in New Zealand; gives analyses and discusses the ehemical character of the waters from these springs and the deposits which they form.

Eleventh Annual Report (1899-90). Part II. Irrigation. 1891.395 pp., 30 pls. and maps. $\$ 1.25$.

*(A 11 II c) Includes a section on artesian irrigation on the Great Plains (pp. 260-278), in which the limitations imposed by quantity, head, and cost are discussed, and irrigation by means of artesian wells in various countries is described. Records are given of wells in Kansas, Minnesota, Montana, Nebraska, North Dakota, South-Dakota, and T $\bullet$ xas. 
Thirteenth Annual Report (1891-92). Part III. Irrigation. 1893. 486.pp., 77 pls. $\$ 1.85$.

The following papers relate in part to ground water:

*(A 13 III a) Water supply for irrigation, by F. H. Newell (pp. 7-99, Pls. CVIII-CX). Includes a brief discussion on "subsurface waters" (pp. 28-30), giving statistics on artesian wells and irrigation with well water in the United States in 1890, and commenting accurately on quantit $y$, ocourrence, and discharge of water in desert valleys.

*(A 13 II b) American irrigation engineering, by H. M. Wilson (pp. 100-349, Pls. CXICXLVI). Includes a chapter on subsurface or ground waters with special reference to irrigation (pp. 326-346), in which are discussed artesian and pumped wells, underflow dams, infitration galleries, and subirrigation.

Fourteenth Annual Report (1892-93). *Part II. Accompanying papers. 1893.597 pp., 73 pls. $\$ 2.10$.

The following papers relate to ground water:

*(A 14 II a) Potable waters of the eastern United States, by W J McGee (pp. 1 to 47). Discusses cistern water, stream waters, and ground watefs, including mineral springs and artesian wells.

*(A 14 II b) Natural mineral waters of the United States, by A. C. Peale (pp. 49-88, Pls. III and IV). Discusses the history, origin, flow, classification, and geographic distribution of mineral and thermal springs, the chemical composition and analysis of spring waters, and the utilization of mineral waters. Gives a list of American mineral spring resorts.

Sixteenth Annual Report (1894-95). *Part II. Papers of an economic character. 1895. 598 pp., 43 pls. $\$ 1.25$.

The following papers relate in part to ground water:

*(A 16 I e) The public lands and their water supply, by F. H. Newell (pp. 457-533, Pls.

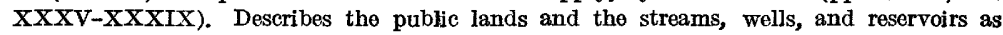
sources of water supply; contains a brief but comprehensive and farsighted discussion of the ground-water resources of the West (pp. 499-502); includes brief notes on ground water in Arizona, California, Colorado, Idaho, Kansas, Nebraska, New Mexico, North Dakota, Oklam homa, Orogon, South Dakota, Texas, Utah, and Washington (pp. 504-533).

(A 16 пf) Water resources of a portion of the Great Plains, by Robert Hay (pp. 535-588, Pls. XL-XLII). Describes an indefinite area that liess on both sides of the east boundary of Colorado and extends from the vicinity of Smoky Hill River northward to the North Platte, thus comprising parts of Colorado, Nebraska, and Kansas. Discusses the lakes, streams, and springs of the area, the underflow of the river bottoms, and the water-bearing strata beneath the higher lands, the source, quantity, and rate of percolation of ground water, the wells in the valleys and on the uplands, the unsuccessful wells, artesian conditions, "blowing" wells and the temperatures of the well waters. Describes briefly the topography and geology of the region and the utilization of the water supply.

Seventeenth Annual Report (1895-96). *Part II. Economic geology and hydrography. 1896. 864 pp., 113 pls. $\$ 2.35$.

The following papers relate to ground water:

(A 17 IIf) The underground water of the Arkansas Valley in eastern Colorado, by G. K. Gilbert (pp. 551-601, Pls. LVI-LXVII). Relates to an indefinite region adjacent to Arkansas River, in southeastern Colorado. Describes the topography, geology, artesian conditions, and intake areas of the region, the water in the Dakota sandstone, in the upland sands, beneath the terraces, and in the dune sands, and the underflow of rivers and creeks. Includes a sketch map showing the depths to artesian water. See also Professional Paper 52. .

(A 17 II g) Preliminary report on artesian waters of a portion of the Dakotas, by N. H. Darton (pp. 603-694, Pls. LXIX-CVII). Covers the part of South Dakota lying east of the 101st meridian and the part of North Dakota lying east of the 101st meridian and south of the 47th parallel. Gives an outline of the geologic relations, describes the water horizons and the extent of the artesian waters, gives detailed information by counties concerning wolls and prospects for obtaining wells, discusses the origin, quantity, head, and quality of the artesian waters and their use for doveloping power, gives data by counties regarding irrigation with artesian water and directions as to the construction and management of artesian wells. Ineludes maps showing the areas of artesian flow supplied from the Dakota sandstone and from glacial drift, respectively, the height to which the artesian water will rise, the depths to principal artesian horizon of the Dakota sandstone, and contours of the surface of the bedrock.

*(A $17 \mathrm{I} \mathrm{h}$ ) The water resources of Illinois, by Frank Leverett (pp. 695-849, Pls. CVIIICXIII). Describes the topography, drainage, precipitation, run-off, navigable rivers, water powers, wells supplying water for eities, villages, and rural districts, wells in alluvium, glacial drift, and Tertiary and Paleozoic formations, and flowing wells. Contains tabulated well data and water analyses, and includes maps of nlinois and eastern Indiana showing the distrnbution of Pleistocene deposits, the relation of glacial drift to ground-water supplies, the elevation of the St. Peter sandstone, and areas in which there are flowing wells supplied by glacial drift. Also contains a map of Wisconsin and northern Illinois showing the main intake areas of the "Potsdam" and st. Peter sandstones. 
Eighteenth Annual Report (1896-97). *Part II. Papers chiefly of a theoretic nature. 1897. 653 pp., 105 pls. $\$ 1.65$.

The following paper relates to ground water:

1

*(A $18 \mathrm{II}$ b) Geology of portions of the Edwards Plateau and Rio Grande Plain adjacent to Austin and San Antonio, Tex., with special reference to the occurrence of artesian and other underground waters, by R. T. Hill and T. W. Vaughan (pp. 193-322, Pls. XXI-LXIV.) Describes the geography, geology, water-bearing formations, springs, and flowing and nonflowing wells. Discusses the artesian conditions, the probable identity of source of artesian and fissurespring waters, and the quality of the artesian water. Contains water analyses and includes a map of the vicinity of Austin showing the relations of the springs and artesian wells to the geology.

Eighteenth Annual Report (1896-97). *Part IV. Hydrography. 1897.756 pp., 102 pls. $\$ 1.75$.

The following papers relate to ground water:

*(A 18 IV b) The water resources of Indiana and Ohio, by Frank Leverett (pp. 419-560, Pls. XXXIII-XXXVII). Describes the drainage systems, the ground-water conditions, the flow- . ing and nonflowing wells in glacial drift and rock formations, and the mineral springs. Contains numerous well records and water analyses. Gives data regarding water supplies for cities and villages derived from surface and underground sources. Includes maps of Indiana and Ohio showing the Pleistocene deposits, the older geologic formations, and the relation of groundwater supplies to the depth of the glacial drift.

*(A 18 IV c) New development in well boring and irrigation in eastern South Dakota, by N. H. Darton (pp. 561-616, Pls. XXXVIII-XLVII). Describes the progress that was made in 1896 in drilling wells and in irrigating with artesian waters in Aurora, Beadle, Bonhomme, Brule, Buffalo, Charles Mix, Davison, Douglas, Hanson, Hutchinson, Jerauld, Sanborn, Spink, and Yankton counties, and in areas west of Missouri River. Discusses the temperature, pressure, and flow of the artesian waters, the extent of the artesian basin, and the position of the bedrock. Gives analyses of waters from Missouri River and from artesian wells in the Sanborn basin. Inchudes maps showng the rate of increase of temperature in wells with depth, contours of the bedrock surface, and flow of wells.

Nineteenth Annual Report (1897-98). *Part II. Papers chiefly of a theoretic nature. 1899. 958 pp., 172 pls. $\$ 2.65$.

The following papers relate to ground water:

*(A $19 \mathrm{n} \mathrm{b)} \mathrm{Principles} \mathrm{and} \mathrm{conditions} \mathrm{of} \mathrm{the} \mathrm{movements} \mathrm{of} \mathrm{ground} \mathrm{water,} \mathrm{by} \mathrm{F.} \mathrm{H.} \mathrm{King}$ (pp. 59-294, Pls. VI-XVII). Discusses the quantity of water stored in soil, in sandstone, and in other rocks, the depth to which ground water penetrates, the gravitational, thermal, and capillary movements of ground water, and the configuration of the water table. Gives the results of tests made by the author and.by earlier investigators of the flow of air and water through rigid, porous media and through sands, sandstones, and silts. Summarizes these results and draws conclusions regarding the relation of velocity to pressure. Discusses also the influence of form, diameter, and arrangement of sand grains on velocity, the methods of determining diameters of sand grains, the growth of rivers, the rate of seepage into filtration ditches, the interference of wells, and related subjects.

*(A $19 \mathrm{I} \mathrm{c)} \mathrm{Theoretical} \mathrm{investigation} \mathrm{of} \mathrm{the} \mathrm{motion} \mathrm{of} \mathrm{ground} \mathrm{waters,} \mathrm{by} \mathrm{C.} \mathrm{S.} \mathrm{Slichter}$ (pp. 295-384, Pl. XVII). A mathematical discussion of the laws governing the movements of ground water, the discharge of flowing wells, and the mutual interference of wells. Includes a bibliography on the motion of ground waters and related topics.

Nineteenth Annual Report (1897-98). * *Part IV. Hydrography. 1898.814 pp., 118 pls. $\$ 1.85$.

The following papers relate to ground water:

*(A $19 \mathrm{Iv} \mathrm{b)} \mathrm{The} \mathrm{rock} \mathrm{waters} \mathrm{of} \mathrm{Ohio,} \mathrm{by} \mathrm{Edward} \mathrm{Orton} \mathrm{(pp.} \mathrm{633-717,} \mathrm{P1s.} \mathrm{LXXI-LXXIII.)}$ Describes the principal geologic formations of ohio and the waters which they yield; gives detailed information regarding the water supplies of many cities and villages; discusses the flowing wells in various localities, including those in the preglacial channels of Allen, Auglaize, and Mercer counties; and includes a number of water analyses.

*(A. 19 Iv c) Preliminary report on the geology and water resourbes of Nebraska west of the 103d meridian, by N. H. Darton (pp. 727-785, Pls. LXXIV-CXVIII). Describes the general geology of Nebraska, and the topography, geology, and water howzons of the area covered by the reports. Gives information on springs, streams, irrigation, climate, and timber, and of elevations of various points. Includes a general geologic map of Nebraska and moredetailed maps of the portion of the State west of the 103d meridian, showing the geology and the groundwater conditions. Reprinted as Professional Paper 17.

$56122^{\circ}-18-$ WsP $427-3$ 
Twenty-first Annual Report (1899-1900). *Part IV. Hydrography. 1901. 768 pp., 156 pls. $\$ 2.35$.

The following papers relate in part to ground water:

*(A $21 \mathrm{rv}$ a) Report of progress of stream measurements for the calendar year 1899, by F. H. Newell (pp. 9-488, Pls. I-I.VII). Includes brief notes on ground-water supplies at Kearney, Nebr. (pp. 216-217), Alamosa, Colo. (p. 265 and Pl. IV, A), San Pedro Valley, Ariz. (pp. 352-353 and PI. XXXI, A), Mohave Valley, Cal. (p. 472, and Pl. LII), Los Angeles River valley, Cal. (p. 474), Lytle Creek valley, Cal. (pp. 481-482), and Mission Valley, Cal. (p. 486). The plates referred to are photographs of flowing wells or other ground-water features.

* (A $21 \mathrm{IV}$ b) Preliminary description of the geology and water resources of the southern half of the Black Hills and adjoining regions in South Dakota and Wyoming, by N. H. Darton (pp. 489-599, Pls. LVIII-CXII). Covers an area comprising about 5,500 square miles in southwestern South Dakota and the adjoining portion of Wyoming. Describes the topography, geology, water horizons, wells, surface waters, irrigation, soil, mineral resources, climate; and timber of the area. Includes maps showing the geology, the depths to the Dakota sandstone, and other ground-water conditions.

*(A 21 Iv $c$ ) The High Plains and their utilization, by W. D. Johnson (pp. 601-741, Pls. CXIII-CLVI). Descrihes the area lying in an irregular belt about midway across the long eastward slope of the Great Plains and including parts of Wyoming, Nebraska, Colorado, Kansas, New Mexico, and Texas. Gives a comprehensive description of the physiographic features and Tertiary deposits of the region and a critical discussion of their interpretation. Discusses precipitation, climate, and the use of streams and storm waters for irrigation. Describes the artesian conditions, with special reference to the Meade artesian basin, and explains the principles of artesian and other ground waters. Concluded in the Twenty-second Annual Report, Pt. IV.

Twenty-first Annual Report (1899-1900). *Part VII. Geography and geology of the Black and Grand prairies, Tex., with detailed descriptions of the Cretaceous formations and special reference to artesian waters, by R. T. Hill. 1901. $666 \mathrm{pp} ., 71$ pls. $\$ 1.90$.

Gives a general description of the geography of a region including Texas, Oklahoma, and New Mexico east of the Rio Grande, and describes in more detail the geography and geology of the Black and Grand prairies. Discusses the principles governing artesian and other ground waters, the artesian systems of Texas, and the quality of the waters of these systems. Describes the artesian conditions by counties and gives analyses. Includes maps showing the geology, the locations of artesian wells, and the outcrop of, depths to, and areas of artesian flow from the Trinity, Paluxy, and Woodbine formations.

Twenty-second Annual Report (1900-1901). Part IV. Hydrography. 1902. 690 pp., 65 pls. $\$ 2.20$.

The following paper relates to ground water:

*(A 22 Iv c). The High Plains and their utilization, by W. D. Johnson (pp. 631-669, Pls. LI-LXV). This is the concluding part of the paper on the High Plains and their utilization begun in the Twenty-first Annual Report, Part IV. It discusses the occurrence of water, consolidated and unconsolidated formations, the origin and level of the ground water of the High Plains, the utilization of ground water for stock raising and irrigation, and methods of constructing wells.

\section{MONOGRAPHS.}

25. The glacial Lake Agassiz, by Warren Upham. 1896.658 pp., 38 pls. $\$ 1.70$.

Contains a chapter (pp. 523-582) on "Artesian and common wells of the Red River Valley," which diseusses the sources of artesian water, the fresh waters in the drift sheets, the saline and alkaline waters in the Dakota sandstone, and the use of artesian water for irrigation; contains analyses of waters from wells, streams, and lakes in Red River Valley and the adjoining region; and gives notes on wells in Clay, Kittson, Marshall, Norman, Polk; Traverse, and Wilkin counties, in Minnesota; in Cass, Grand Forks, Pembina, Richland, Traill, and Walsh counties, in North Dakota; and in a part of the area covered by Lake Agassiz, in Manitoba.

- The monograph includes numerous maps relating to the Pleistocene geology of the region and a map (Pl. XXXVII) showing the distribution and depths of artesian wells in glacial drift and bedrock. 
27. Geology of the Denver Basin in Colorado, by S. F. Emmons, Whitman Cross, and G. H. Eldridge. 1896.556 pp., 31 pls. $\$ 1.50$.

Contains a discussion of the water in the Pleistocene deposits (pp. 272, 273) and a section on artesian wells (pp. 401-465). Discusses the history of artesian-water developments in Colorado, the water-bearing horizons, the artesian structure, the quanticy of artesian water, and the yield and decrease in yield of flowing wells. Includes three analyses of well waters and maps showing the geology of the region and the original area of artesian flow.

38. The Illinois glacial lobe, by Frank Leverett. 1899.817 pp., 24 pls. $\$ 1.60$.

Includes a chapter (pp. 550-788) on "Wells of Illinois," which contains a general discussion of artesian and other wells, a table of municipal water supplies derived from underground sources, and a detailed description of wells and ground-water conditions in practically every county in the State. The monograph includes maps showing the geology, the distribution of wells, the intake areas of "Potsdam" and St. Peter sandstones, and the relation of glacial drift to ground-water supplies.

*47. A treatise on metamorphism, by C. R. Van Hise. 1904.1286 pp., 13 pls.

This comprohensive treatise deals in much detail with the chemical and physical laws governing ground water, especially in the following sections: "Chemical and physical principles controlling the action of ground water" (pp. 65-123), "Circulation and work of ground water" (pp. 123-158), "The belt of weathering" (pp. 411-429), "The belt of cementation" (pp. 566594), and "Work of aqueous solutions in segregating ores" (pp. 1072-1198).

\section{PROFESSIONAL PAPERS.}

*17. Preliminary report on the geology and water resources of Nebraska west of the 103d meridian, by N. H. Darton. 1903. 69 pp., 43 pls. $25 \mathrm{c}$.

Reprint of a paper in the Nineteenth Annual Report, Part IV, with slight changes.

*32. Preliminary report on the geology and underground-water resources of the central Great Plains, by N. H. Darton. 1905.433 pp., 72 pls. $\$ 1.80$.

Covers South Dakota, Nebraska, central and western Kansas, eastern Colorado, and eastern Wyoming. Describes the geography, geology, and water horizons; gives Aeep-well data and well prospects by counties; also describes other mineral resources. Includes maps showing the geology, locations of deep wells, structure of the Dakota sandstone, depths to this sandstone, head of artesian water, and areas of artesian flow.

*44. Underground-water resources of Long Island, N. Y., by A. C. Veatch, C. S. Slichter, Isaiah Bowman, W. O. Crosby, and R. E. Horton. 1906. 394 pp., 34 pls. $\$ 1.25$.

Describes the geologic formations, the source and occurrence of ground water, and the conditions necessary to obtain flowing wells; gives data in regard to the springs, streams, ponds, lakes, artesian and other deep wells, the water table and its fluctuations, blowing wells, and waterworks; contains records of the rate of movement of the ground water and results of sizing and flltration tests; gives well records and notes concerning representative wells. Includes water analyses and maps showing the geology, convours of the water table, and locations of wells and waterworks.

*46. Geology and underground-water resources of northern Louisiana and southern Arkansas, by A. C. Veatch. $1906.422 \mathrm{pp}, 51$ pls. $\$ 1.50$.

Covers Louisiana north of the 31st parallel, about the southern half of Arkansas, and adjacent areas in Texas, Oklahoma, and Mississippi. Describes the physiography and geology of the region, the principles governing ground waters and thoir application to this region, the waterbearing formations with reference to springs and to the artesian pressure, quality, and availability of the water, the occurrence of mineral waters, the hygienic value of deep-well waters, and methods and costs of constructing wells. Contains a description of the ground-waterconditions in each county and tables of well data arranged by counties, with notes giving well sections and water analyses. Contains also a dictionary of altitudes, arranged by countios. Includes maps showing the geology of the region, the structure contours of the Nacatoch and Bingen formations, areas of artesian flow supplied by these formations, depths to the Sabine and Cockfield sands, and areas of artesian flow supplied by thesa sands. 
*51. Geology of the Bighorn Mountains, by N. H. Darton. 1906.129 pp., 47 pls.

Describes the geology of the region in detail and contains a brief discussion (pp. 119 and 120) of the probable depths and water-bearing conditions of the principal sandstones. Includes a geologic map.

*52. Geology and underground waters of the Arkansas Valley in eastern Colorado, by N. H. Darton. 1906.90 pp., 28 pls.

Describes the geology of the greater part of the drainage basin of Arkansas River in Colorado, the source, depths, head, areas of artesian flow, quantity, and quality of the water in the Dakota sandstone, and the occurrence and quality of the waters in the Red Beds, Morrison formation, Laramie and associated formations, later Tertiary deposits, and dune sands. Includes numerous well sections, several water analyses, and maps showing the geology, dépths to Dakota sandstone, areas of artesian flow, and areas not underlain by Dakota sandstone. A preliminary report on the same region, by G. K. Gilbert, was published in the Seventeenth Annual Report, part 2, 1896, pp. 1-51.

*53. Geology and water resources of the Bighorn Basin, Wyo., by C. A. Fisher. 1907. 72 pp., 16 pls.

Describes the geography, geology, water-bearing formations, irrigation developments, mineral waters, and other mineral resources of the basin. Includes a geologic map.

*56. Geography and geology of a portion of southwestern Wyoming, with special reference to coal and oil, by A. C. Veatch. 1907.178 pp., 26 pls. $60 \mathrm{c}$.

Covers the southwest corner of Wyoming and a small adjacent portion of Utah. Gives a detailed description of the geslogy and a brief discussion of the water-bearing formations. Includes a geologic map with structure contours.

*65. Geology and water resources of the northern portion of the Black Hills and adjoining regions in South Dakota and Wyoming, by N. H. Darton. 1909. 105 pp., 24 pls. $40 \mathrm{c}$.

Describes the geology of the sedimentary rocks and discusses their mineral resources, including their water supplies. Contains also information concerning the timber, climate, and surface waters available for irrigation and stock raising. Includes maps showing the geology, outcrops of and depths to principal water-bearing formations, and areas of artesian flow.

*90. Shorter contributions to general geology, 1914; David White, chief geologist. 1915. 199 pp., 21 pls. 40c.

Issued also in separate chapters. The following paper relates in part to ground water:

(h) A deep well at Charleston, S. C., by L. W. Stephenson, with a report on the mineralogy of the water, by Chase Palmer (pp. 69-94).

94. Economic geology of Gilpin County and adjacent parts of Clear Creek and Boulder counties, Colo., by E. S. Bastin and J. M. Hill. 1917. 379 pp., 23 pls.

Discusses, on pages 134 to 152 , the relation of ground water to ore deposition, especially the relation of the water table to the downward enrichment of gold, silver, and copper ores. Contains, however, almost no data regarding ground water.

95. Shorter contributions to general geology, 1915; David White, chief geologist. 1916. 120 pp., 7 pls: $20 \mathrm{c}$.

Issued also in separate chapters. The following paper relates in part to ground water:

*(a) The composition of muds from Columbus Marsh, Nev., by W. B. Hicks (pp. 1-11). Gives data in regard to shallow wells on Columbus Marsh.

\section{BULIETINS.}

*32. Lists and analyses of the mineral springs of the United States (a preliminary study), by A. C. Peale. 1886. $235 \mathrm{pp}$.

Defines mineral waters, gives lists of springs by States, and contains 819 analyses of spring waters.

*47. Analyses of waters of the Yellowstone Nafional Park, with an account of the methods of analysis employed, by F. A. Gooch and J. E. Whitfield. 1888. $84 \mathrm{pp}$.

Describes methods used in analyzing natural waters and contains analyses of 43 geyser, spring, and surface waters in Yellowstono National Park. 
*108.. A geological reconnaissance in central Washington, by I. C. Russell. 1893. $108 \mathrm{pp}, 12 \mathrm{pls}$. $15 \mathrm{c}$.

Describes the main geologic features of a region lying approximately between the 119th and 121st meridians and between the 46th and 48th parallels. Gives conclusions regarding prospects for artesian wells and includes a sketch geologic map. This bulletin is largely superseded by Water-Supply Papers 55, 118, and 316.

*131. Report of progress of the division of hydrography for the calendar years 1893 and 1894 , by F. H. Newell. $1895.126 \mathrm{pp}$. $15 \mathrm{c}$.

Gives the discharge of Barton Spring, near Austin, Tex., as 17 second-feet on November 13,1894 (p. 92). Contains records of wells collected by Robert Hay in Nebraska, Colorado, and Kansas (pp. 92-126).

*138. Artesian-well prospects in the Atlantic Coastal Plain region, by N. H. Darton. 1896. $232 \mathrm{pp}$., 19 pls.

Describes brieffy the geologic structure and the ground-water conditions in the Atlantic Coastal Plain. Covers the Coastal Plain areas of New York, New Jersey, Delaware, Maryland, District of Columbia, Virginia, North Carolina, South Carolina, and eastern Georgia, giving for each of these States a discussion of the geologic relations and well prospects, tabulated data regarding deep wells, and notes giving well sections, water analyses, and other detailed information not found in the tables. Includes maps and sections showing ground-water conditions.

*140. Report of progress of the division of hydrography for 1895, by F. H. Newell. 1896. $356 \mathrm{pp}$. $25 \mathrm{c}$.

Gives descriptions and discharge records for the following springs in Texas: San Marcos Spring, San Antonio and San Pedro springs, Las Moras Spring, San Felipe Springs, and Barton Springs (pp. 83-86). Contains data on seepage and evaporation in Nebraska and Kansas (pp. 347-350).

*164. Reconnaissance in the Rio Grande coal fields of Texas, by T. W. Vaughan. 1900. $100 \mathrm{pp} ., 11$ pls. $20 \mathrm{c}$.

Contains brief notes on artesian wells in the vicinities of Eagle Pass and Carrizo springs. See especially pp. 25, $50-52$.

*199. Geology and water resources of the Snake River Plains of Idaho, by I. C. Russell. 1902. $192 \mathrm{pp} ., 25 \mathrm{pls}$. $25 \mathrm{c}$.

Gives a general sketch of the geography and geology of an indefinite region in Idaho adjacent to Snake River. Discusses artesian and other ground-water conditions and includes a meager amount of well data.

*227. The United States Geological Survey, its origin, development, organization, and operations. 1904.205 pp., 9 pls. 25 c.

Includes a brief account (pp. 84-88) of the Division of Hydrology (ground water) and of the investigations relating to ground water made by the United States Geological Survey prior to 1904 .

*238. Economic geology of the Iola quadrangle, Kans., by G. I. Adams, Erasmus Haworth, and W. R. Crane. 1904.83 pp., 11 pls. $25 \mathrm{c}$.

Describes the geology and contains a geologic map of the quadrangle. Discusses briefly the water-bearing formations and the quality of their waters (p. 77).

252. Preliminary report on the geology and water resources of central Oregon, by I. C. Russell. 1905.138 pp., 24 pls. $15 \mathrm{c}$.

Covers an indefinite region that lies partly in the Great Basin and partly in the basin of Deschutes River, and includes portions of Malheur, Harney, and Crook counties. Describes the geography and geology of the region, gives meager data regarding the surface and ground waters, discusses briefiy the artesian conditions in the Deschutes basin, and makes suggestion concerning records of artesian wells.

*264. Record of deep-well drilling for 1904, by M. L. Fuller, E. F. Lines, and A. C. Veatch. $1905.106 \mathrm{pp} .{ }^{\circ} 10 \mathrm{c}$.

Discusses the importance of accurate well records to drillers, to owners of oil, gas, and water wells, and to geologists. Describes methods of work. Gives tabulated records of wells in 39 States and Territories, and detailed records of wells in California, Colorado, Illinois, Towa, Kansas, Mississippi, Missouri, New Jersey. New York, Ohio, Pennsylvania, Texas, and West Virginia. 
265. Geology of the Boulder district, Colo, by N. M. Fenneman. 1905. 101 pp., 5 pls. $15 \mathrm{c}$.

Describes the geology of a rectangular area 16 mules north and south by 9 miles east and west, in the southwestern part of which is situated the city of Boulder. Discusses briefly (pp. 67-69) the flowing wells and water-bearing formations, including the Dakota sandstone. Contains a geologic map of the area.

*282. Oil fields of the Texas-Louisiana Gulf Coastal Plain, by N. M. Fenneman. 1906. 146 pp., 11 pls.

Includes well records and discussions of phenomena related to wells, drilling methods, and movements, temperatures, and salinity of ground waters.

*285. Contributions to economic geology, 1905; S. F. Emmons and E. C. Eckel, geologists in charge. $1906.506 \mathrm{pp} ., 13 \mathrm{pls}$.

Issued also in separate chapters. The following paper contains information on ground water: * $(f)$ Coal and oil in southern Uinta County, Wyo., by A. C. Veatch (pp. 331-353, Pls. XXII). Describes the geology of, an area that occupies Tps. 12 to $23 \mathrm{~N}$., Rs. 115 to $121 \mathrm{~W}$., inclusive, in Uinta County, Wyo. Contains a geologic map and a brief statement in regard to artesian conditions and prospects (p. 353). This area is covered more fully in Professional Paper 56.

*298. Record of deep-well drilling for 1905, by M. L. Fuller and Samuel Sanford. 1906. 299 pp. $25 \mathrm{c}$.

Gives an account of progress in the collection of well records and samples. Contains tabulated records of wells in 48 States and Territories and detailed records of wells in Alabama, Arizona, Arkansas, California, Colorado, Delaware, Florida, Georgia, Illinois, Indiana, Indian Territory, Iowa, Kansas, Kentucky, Maine, Maryland, Massachusetts, Minnesota, Mississippi, Missouri, Montana, New Jersey, New Mexico, New York, North Carolina, Ohio, Oklahoma, Pennsylvania, South Carolina, South Dakota, Texas, Utah, Washington, West Virginia, and Wisconsin.

*300. Economic geology of the Amity quadrangle, eastern Washington County, Pa., by F. G. Clapp. 1907.145 pp., 8 pls. 30c.

Contains a description of the geology of the quadrangle and a map showing outcrops and structure. Includes brief statements on water supplies and water-bearing formations (pp. 130-134). The quadrangle is covered more fully in Geologic Folio 144, which also contains information on ground water.

*308. A geologic reconnaissance in southwestern Nevada and eastern California, by S. H. Ball. 1907, 218 pp., 3 pls. $40 \mathrm{c}$.

Contains a brief section on "Hydrology," which discusses streams, springs, tanks, wells, and signs of water (pp. 18-23). Contains descriptions of specific areas which give detailed information regarding springs, wells, and other watering places. Includes a geologic map that also shows watering places and connecting roads.

*319. Summary of the controlling factors of artesian flows, by Myron L. Fuller. 1908. 44 pp., 7 pls. . 10c.

Describes underground reservoirs, the sources of ground water, the confining agents, the primary and modifying factors of artesian circulation, the essential and modifying factors of artesian flow, and typical artesian systems.

*330. The data of geochemistry, by F. W. Clarke. 1908. 716 pp. Superseded by Bulletin 616 .

*350. Geology of the Rangely oil district, Rio Blanco County, Colo., with a section on the water supply, by H. S. Gale. 1908.61 pp., 4 pls. $20 \mathrm{c}$.

Describes the geology and contains a geologic map of an area including approximately Tps. 1, 2, and 3 N., Rs. 101, 102, and 103 W., 6th principal meridian. Discusses the water supplies in this area, including artesian wells (p. 58). 
*352. Geologic reconnaissance of a part of western Arizona, by W. T. Lee, with notes on the igneous rocks of western Arizona, by Albert Johannsen. 1908. 99 pp., 11 pls. $25 \mathrm{c}$.

Describes the geography and geology and contains a geologic sketch map of western Arizona north of longitude $33^{\circ} 30^{\prime}$, including the valley of Colorado River and Hualpai, Big Sandy, Detrital-Sacramento, Williams, and McMullen valleys. Contains a section on water supplies, which includes well data and discussion of ground-water prospects.

364. Geology and mineral resources of the Laramie Basin, Wyo. (a preliminary report), by N. H. Darton and C. E. Siebenthal. 1909. 81 pp., 8 pls. $20 \mathrm{c}$.

Describes the geology and contains a geologic map. Includes a section on ground water (pp. 67-78), in which are given well data and 6 water analyses. A part of the area is coyered by Geologic Folio 173, which also contains information on ground water.

395. Radioactivity of the thermal waters of Yellowstone National Park, by Herman Schlundt and R. B. Moore. 1909.35 pp., 4 pls. $10 \mathrm{c}$.

Describes the apparatus and methods used and presents and discusses the results of the . experiments.

435. A reconnaissance of parts of northwestern New Mexico and northern Arizona, by N. H. Darton. 1910. 88 pp., 17 pls. $35 \mathrm{c}$.

Describes the geology and contains a geologic map of the region. Iucludes data in regard to wells at 24 stations on the Atchison, Topeka \& Santa Fe R. R. (pp. 75-81).

438. Geology and mineral resources of the St. Louis quadrangle, Mo.-Ill., by N. M. Fenneman. 1911. 73 pp., 6 pls. 25c.

Describes the geology and contains a geologic map of a rectangular area 31 miles east and west by 17 miles north and south, including the city of St. Louis. Discusses the water resources, including springs, flowing wells, and deep wells ending in Carbonferous and Ordovician formations (pp. 65-69). The part of this area that lies in Illinois is also covered by Bulletin 5 of the Illinois Geographical Survey. (See S 3.)

447. Mineral resources of Johnstown, Pa., and vicinity, by W. C. Phalen and Lawrence Martin. 1911. $142 \mathrm{pp}$., 7 pls. 25c.

Describes the geology and contains a map of the Johnstown quadrangle showing structure contours. Includes a very hrief description of water supplies and ground-water conditions (pp. 136-137), which is reprinted in Geologic Folio 174, covering the same quadrangle.

*471. Contributions to economic geology, 1910, Part II, Mineral fuels; M. R. Campbell, geologist in charge. 1912 . $663 \mathrm{pp} ., 62 \mathrm{pls} . \$ 1.05$.

Issued also in separate chapters. The following paper contains information on ground water:

*(a) The Powder River oil field, Wyo., by C. H. Wegemann (pp. 56-75). Descrihes the geology and contains a geologic map of a quadrangular area which includes Tps. 40-42 N., R. $81 \mathrm{~W}$., and portions of adjoining townships. Contains brief notes on water supplies, including water-bearing formations (pp. 58, 59).

*479. The geochemical interpretation of water analyses, by Chase Palmer. 1911. $31 \mathrm{pp}$. 5c.

Discusses the expression of chemical analyses and the chemical character and properties of natural waters. Gives a classification of waters based on property values and reacting values, and discusses the character of the waters of certain rivers as interpreted from the analyses. Discusses also the relation of the properties of water to geologic formations, silica in river water, and the character of the water of Mississippi River, the Great Lakes, and St. Lawrence River as indicated by chemical analyses.

491. The data of geochemistry (second edition) by F. W. Clarke. $1911.782 \mathrm{pp}$. Superseded by Bulletin 616 .

506. Geology and mineral resources of the Peoria quadrangle, Ill., by J. A. Udden. 1912. 103 pp., 9 pls. $25 \mathrm{c}$.

Describes the physiography and geology of a 15-minute quadrangle that includes the cities of Peoria and Pekin. Contains detailed well records and a section on water resources, in which are discussed the water-bearing formations, and the quality, quantity, head, temperature, and use of the artesian waters (pp. 90-97). Includes 8 chemical analyses and maps showing the geology and locations of artesian wells. 
*529. The enrichment of sulphide ores, by W. H. Emmons. 1913.260 pp. $20 \mathrm{c}$.

Contains a section on underground circulation(pp. 26-31), in which are aiscussed the " vadose" and deeper circulation and the region of nearly stagnant waters. Contains also 37 analyses of mine waters with disctussion (pp. 60-74) and a discussion of chemical changes in descending sulphate waters (pp. 89-91) and related subjects. See also Bulletin 625 .

*530. Contributions to economic geology, 1911, Part I, Metals and nonmetals except fuels; Waldemar Iindgren, chief geologist. $1913.400 \mathrm{pp} ., 7$ pls. $30 \mathrm{c}$.

Issued also in separate chapters. The following papers relate in part to ground water:

*(b) The occurrence of potash salts in the bitterns of the eastern Unites States, by W. C. Phalen (pp. 313-329). Includes brief statements in regard to the stratigraphic occurrence and origin of salt water in New York, Michigan, Ohio, West Virginia, Kansas, and Louisiana; also contains analyses.

(r) Salines in Silver Peak Marsh, Nev., by R. B. Dole (pp. 330-345). Includes records of 14 borings, 8 to 55 feet deep, a description of methods used in boring, and analyses of water from these holes and from wells and springs in the vicinity.

*531. Contributions to economic geology, 1911, Part II, Mineral fuels; M. R. Campbell, geologist in charge. 1913.361 pp., 24 pls. $45 \mathrm{c}$.

Issued also in separate chapters. The following papers contain information on ground water. *(c) Geology and petroleum resources of the De Beque oil field, Colo., by E. G. Woodruff (pp. $54-68, \mathrm{Pl}$. VI). Contains a description of the geology and a geologic map of a square area covering Tps. 7 and 8 S., Rs. 97 and 98 W., in the vicinity of De Beque, in Mesa and Garfield'countles. Includes a brief statement on artesian water in the area (p. 61).

*(d) Geologic structure of the Punxsutawney, Curwensville, Houtzdale, Barnesboro, and Patton quadrangles, central Pennsylvania, by G. H. Ashley, and M. R. Campbell (pp. 69-89, Pls. VII-VIII). Discusses the geologic structure of the five quadrangles named and includes a map showing structure contours. It contains a brief statement in regard to shallow and deep wells and artesian prospects (pp. 88-89). The ground water in the Barnesboro and Patton quadrangles is also briefly described in Geologic Folio 189, and the ground water in these two quadrangles and in the Curwensville quadrangle is briefly described in Water Supply Paper 110.

*540. Contributions to economic geology, 1912, Part I, Metals and nonmetals except fuels; David White, chief geologist. $1914.563 \mathrm{pp} ., 11 \mathrm{pls} . \quad 45 \mathrm{c}$.

Issued also in separate chapters. The following papers contain information on ground water:

* $(n)$ Prospecting for potash in Death Valley, Cal., by H. S. Gale (pp. 407-415). Includes detailed sections of five wells, 30 to 70 feet deep, with data in regard to their waters, practically all of which are salty, as is shown by the analyses given.

Potash tests at Columbus Marsh, Nev., by H. S. Gale (pp. 422-427). Includes detailed sections of two wells, 32 and 82 feet deep, respectively, with data in regard to their waters, some of which are not salty.

$*(p)$ Potash in western saline deposits, by J. H. Hance (pp. 457-469). Gives total solids in waters from various wells and springs in the drainage basins of Railroad Valley, Fourmile Flat, and Dixie Salt Marsh, in Nevada, and the record of a 305-foot well at Adamana, Ariz.

*541. Contributions to economic geology, 1912, Part II, Mineral fuels; M. R. Campbell, geologist in charge. 1914 . $532 \mathrm{pp}, 29$ pls. $50 \mathrm{c}$.

Issued also in separate chapters. The following paper contains information on ground water: (d) Oil and gas near Green River, Grand County, Utah, by C. T. Lupton (pp. 115-133, P1. VI). Describes the geology and contains a geologic map of an area of about 300 square miles southeast of the town of Green River. Contains meager data in regard to wells, water supplies, and artesian conditions (pp. 117-123).

*543. Geology and geography of a portion of Lincoln County, Wyo., by A. R. Schultz. 1914. $141 \mathrm{pp} ., 11 \mathrm{pls}$. $50 \mathrm{c}$.

Describes the geology and contains a geologic map of an area in the central part of Lincoln County, between Green River and the Salt River Range (Tps. 22-39 N., Rs. 113-117 W.). Includes a brief discussion of ground water and artesian prospects (pp. 134, 135).

575. Geology of the Standing Rock and Cheyenne River Indian reservations, North and South Dakota, by W. R. Calvert, A. L. Beekly, V. H. Barnett, and M. A. Pishel, 1914. 49 pp., 8 pls. $15 \mathrm{c}$.

Covers an area lying west of Missouri River, north of Cheyenne River, and south of Cannonball River, and extending westward to 102d meridian. Describes the geology and contains a geologic map of the area. Includes a brief discussion of the water in the Dakota and Fox Hills sandstones and in other formations (pp. 24-25). 
*582. Mineral deposits of the Santa Rita and Patagonia mountains, Ariz., by F. C. Schrader, with contributions by J. M. Hill. 1915.373 pp., 25 pls. 50 c.

Includes a brief statement in regard to water supplies from both surface and underground sources (pp. 364-367).

606. Origin of the zinc and lead deposits of the Joplin region, Missouri, Kansas, and Oklahoma, by C. E. Siebenthal. 1915.283 pp., 11 pls. $25 \mathrm{c}$.

A theoretical treatise which relates to underground cireulation in the Ozark region. Discusses artesian circulation and flowing wells (pp. 33-37), geochemical interpretation of water analyses, and acidity, neutrality, and alkalinity of natural waters (pp. 81-88). Reviews, discusses, and classifies analyses of zinc-bearing and related waters from various parts of the United States and from foreign countries (pp. 88-155).

616. The data of geochemistry (third edition), by F. W. Clarke. 1915. $821 \mathrm{pp}$. 45c.

Earlier editions were published as Bulletins 330 and 491. Contains a discussion of the statement and interpretation of water analyses, and a chapter on "Mineral wells and springs" (pp. 179-216). Discusses the definition and classification of mineral waters, changes in the composition of water, deposits of calcareous, ocherous, and siliceous materials made by water, vadose and juvenile waters, and thermal springs in relation to volcanism. Describes the different kinds of ground water and gives typical analyses. Includes a brief bibliography of papers containing water analyses.

618. Geology and underground water of Luna County, N. Mex., by N. H. Darton. 1916. 188 pp., 13 pls.

Describes the geography and geology, the mineral resources, the water supplies from streams, springs, and wells, and the irrigation development from surface and ground waters. Discusses the source, quantity, and quality of the ground waters and the extent of the waterbearing strata and gives well data by townships. Includes maps showing the geology, the contours of the water table, and the depths to ground water.

*621. Contributions to economic geology, 1915, Part II, Mineral fuels; M. R. Campbell and David White, geologists in charge. 1916. 375 pp., 25 pls. 60c.

Issued also in separate chapters. The following chapters contain information on ground water:

* (e) A reconnaissance in Palo Pinto County, Tex., with special reference to oil and gas, by C. H. Wegemann (pp. 51-59). Gives a brief description of the geology of the county with a note on the prospects of obtaining water of good quality from deep sources.

*(l) Oil and gas near Basin, Big Horn County, Wyo., by C. T. Lupton (pp. 157-190, Pl. XVII). Describes the geology and contains a geologic map of parts of Tps. 50-52 N., Rs. 92 and $93 \mathrm{~W}$. Includes a brief description of the water supplies and of the water-bearing sand with a table giving percentages of oil and gas' wells that obtained water in each of these sand strata (pp. 164-166). It also includess well records that contain some data in regard to water (pp. 186-189).

625. The enrichment of ore deposits, by W. H. Emmons. 1917. 530 pp., 7 pls. This paper is a revision of Bulletin 529 with a somewhat enlarged scope.

627. The lignite field of northwestern South Dakota, by D. E. Winchester, C. J. Hares, E. R. Lloyd, and E. M. Parks. 1916.169 pp., 11 pls. $25 \mathrm{c}$.

Describes the geology and contains geologic maps of Harding and Perkins counties. Describes the drainage and water supply and contains a small amount of data on deep wells not given in Water-Supply Paper 227.

628. Geology and coal resources of Castle Valley, in Carbon, Emery, and Sevier counties, Utah, by C. T. Lupton. $1916.88 \mathrm{pp} ., 12 \mathrm{pls.} 20 \mathrm{c}$.

Describes the geology and contains a geologic map of an area lying between the Wasatch Plateau and the San Rafael Swell, in east-central Utah, and extending from the vicinity of Mounds, on the Denver \& Rio Grende Railroad, southwestward about 80 miles. Describes the drainage and water resources,including the prospects of flnding water in theDakota sandstone and underlying McElmo formation. 
641. Contributions to economic geology, 1916, Part II, Mineral fuels; David White, G. H. Ashley, and M. R. Campbell, geologists in charge.

Issued also in separate chapters. The following chapters contain information on ground water:

(b) The oll and gas geology of the Foraker quadrangle, Osage County, Okla., by K. C. Heald (pp. 17-48, Pls. II-III). Contains, on page 20, a brief statement in regard to ground-water conditions.

(i) Anticlines in central Wyoming, by C. J. Hares (pp. 233-280, Pl. XXIII). Covers nearly 5,000 square miles in Natrona and Fremont countles, west of Casper and southeast of Lander. Contains, on pages 235 and 236, a brief discussion of the water supply, including statements regarding various hot springs, springs of large size, sulphur springs, and other mineral springs, also a statement regarding water-bearing formations and artesian prospects. Includes a geologic map.

647. The Bull Mountain coal fields, Musselshell and Yellowstone counties, Mont., by L. H. Woolsey, R. W. Richards, and C. T. Lupton. 1917. 218 pp., 36 pls.

Gives detailed data regarding water supplies, including ground water, for the following townships: Tps. 5-8 N., R. 24 E.; Tps. 5-8 N., R. 25 E.; Tps. 5-8 N., R. 26 E.; Tps. 5-9 N., R. 27 E.; Tps. 5-9 N., R. 28 E.; Tps. 5-9 N., R. 29 E.; Tps. 5-9 N., R. 30 E.; Tps. 5-9 N., R. 31 E.; T. 8 N., R. 32 E. See pages 16, 17, 65-214.

653. Chemical relations of the oil-field waters in San Joaquin Valley, Cal., by G. S. Rogers. 1917. 119 pp.

Discusses water-bearing sands and dry sands in oil fields, the origin of salt water, and the relation of salt water to the occurrence of oil and to geologic structure in oil fields. Classifies the oil-field waters and gives the distribution of their principal constituents. Discusses the chemical relation between water and the hydrocarbons and the significance of water analyses in prospecting for oil. Gives 80 analyses of ground waters in the Coalinga, Kern River, Lost Hills, McKittrick, Midway, and Sunset oil fields, Cal.

656. Anticlines in the southern part of the Big Horn Basin, Wyo., a preliminary report on the occurrence of oil, by D. F. Hewett and C. T. Lupton. 1917. 192 pp., 32 pls.

Covers a large region in northwestern Wyoming, west of the Big Horn Mountains. Gives detailed data regarding surface waters, springs, wells, and ground-water prospects in the numerous anticlinal areas described in the report. See pages 15, 16, 56-188. Includes a geologic map

- and section.

658. Geologic structure in the Cushing oil and gas field, Okla., and its relation to the oil, gas, and water, by C. H. Beal. 1917. 64 pp., 11 pls.

Discusses, on pp. 39, 44-61, the relation of ground water to bodies of oil and gas and to oil and gas bearing structures. Distinguishes "top water," "bottom water," and "edge water." Discusses the surfaces that form the contacts bétween bodies of ground water and bodies of oil or gas and differentiates these from ordinary water tables. Includes diagram showing movement of water into oil wells. Incidentally gives ground-water data of local value.

661. Contributions to economic geology, 1917, Part II, Mineral fuels; David White, G. H. Ashley, and M. R. Campbell, geologists in charge. 1918. 328 pp., 29 pls.

Issued also in separate chapters. The following chapter contains information on ground water:

(d) The Irvine oil field, Estill County, Ky., by E. W. Shaw (pp. 141-191; Pls. XI-XV). Discusses, on pages 176-179, the relation of ground water to the ofl in this field, the character of so-called "dry sands," the mineral composition of the waters, and the relation of mineral composition to the origin of the water and oil. Includes four analyses of waters from the Estill Springs:

691. Contributions to economic geology, 1918. Part II, Mineral fuels; David White, G. H. Ashley, and M. R. Campbell, geologists in charge.

Issued also in separate chapters. The following chapter contains information on ground water:

(c) Geologic structure of the northwestern part of the Pawhuska quadrangle, Okla., by K. C. Heald (pp. 57-100, Pls. XIII-XV). Covers an area just south of the Kansas line and about 90 miles west of the Missouri line (T. 29 N., Rs. 8 and 9 E., and large parts of T. 28 N., R. 9 E.; T. 29 N., R. 8 E.; and T. 27 N., R. 8 E.). Discusses very briefly, on page 60 , the groundwater conditions and the prospects of obtaining potable supplies in the sandstones penetrated in the oil wells. Contains a brief general statement regarding the quality of the ground waters. 


\section{REPORTS ON MINERAL RESOURCES.}

The report on mineral resources for the calendar years 1883 and 1884 and the reports for each subsequent year contain sections on mineral waters, which give statistics, by calendar years, of production, importation, and exportation, lists of mineral springs, and other information in regard to the trade in the waters, of the United States that are sold for medicinal or table use. The statistics are given largely by States.

The reports on mineral waters for successive years were prepared by the following authors:

1883-1900, inclusive, by A. C. Peale.

1901-1904, inclusive, anonymous.

1905 , by M. L. Fuller.

1906-1909, inclusive, by Samuel Sanford.

1910-1912, inclusive, by G. C. Matson.

1913-1915, inclusive, by R. B. Dole.

1916, by A. J. Ellis.

In addition to the annual statistics, these reports contain the following papers relating to mineral waters:

*(M R 1882) Mineral Resources of the United States, 1882. 1883.813 pp. $50 \mathrm{c}$.

*(a) The divining rod, by R. W. Raymond (pp. 610-626). Gives a vivid historical review and discussion of the so-called "divining rod," which has been supposed to have virtue for finding ground water and other minerals, as well as for detecting criminals, etc.

*(M R 1905) Mineral Resources of the United States, 1905. 1906.1403 pp.

Bibhography of mineral waters, by A. C. Peale (pp. 1308-1312). Includes a general bibliography and a bibliography for the United States, 1903-1905.

*(M R 1911) Mineral Resources of the United States, 1911, Part II, Nonmetals. 1912. 1224 pp., 9 pls. $\$ 1$.

*(n) The concentration of mineral water in relation to therapeutic activity, by R.B.Dole (pp. 1175-1192). Discusses mineral constituents in relation to physiological reactions, minimum doses of inorganic substances, the therapeutic action of certain inorganic radicles, and tolerance for mineral matter in drinking water.

*(M R 1913) Mineral Resources of the United States, 1913, Part II, Nonmetals, 1914. 1617 pp., 9 pls. $80 \mathrm{c}$.

(h) Published analyses of American mineral waters, by R. B. Dole (pp. 433-434). An incomplete bibliography of papers containing analyses of mineral waters.

Radioactivity of mineral waters, by R. B. Dole (pp. 435-440). Gives the radioactivity of 52 well-known waters from springs in Europe and the United States, compares their strength with that of radioactive compounds used in medical practice, and discusses the therapeutic uses of radioactive waters. Includes an incomplete bibliography of publications on radium, radioactivity, and radiotherapy.

(M R 1914) Mineral Resources of the United States, 1914, Part II, Nonmetals, 1916. 1122 pp., 5 pls.

A historical sketch of the mineral-water trade, by R. B. Dole (pp. 215-219). Tabulates and discusses the domestic production and importation from 1883 to 1914 and includes diagrams showing the annual production and the price during this period.

(M R 1916) Mineral Resources of the United States, 1916, Part II, Nonmetals. 1918.

Comparison of American and European mineral waters, by A. A. Chambers. 11 pp. Compares the analyses of certain chalybeate, carbonate, sulphide, chloride, and sulphate spring waters in the United States with the analyses of well-known mineral waters of Europe of similar types. Concludes that the counterparts of European waters can in general be found in this country. Contains a brief bibliography of mineral waters, chiefly those from American springs. 


\section{GEOLOGIC FOLIOS.}

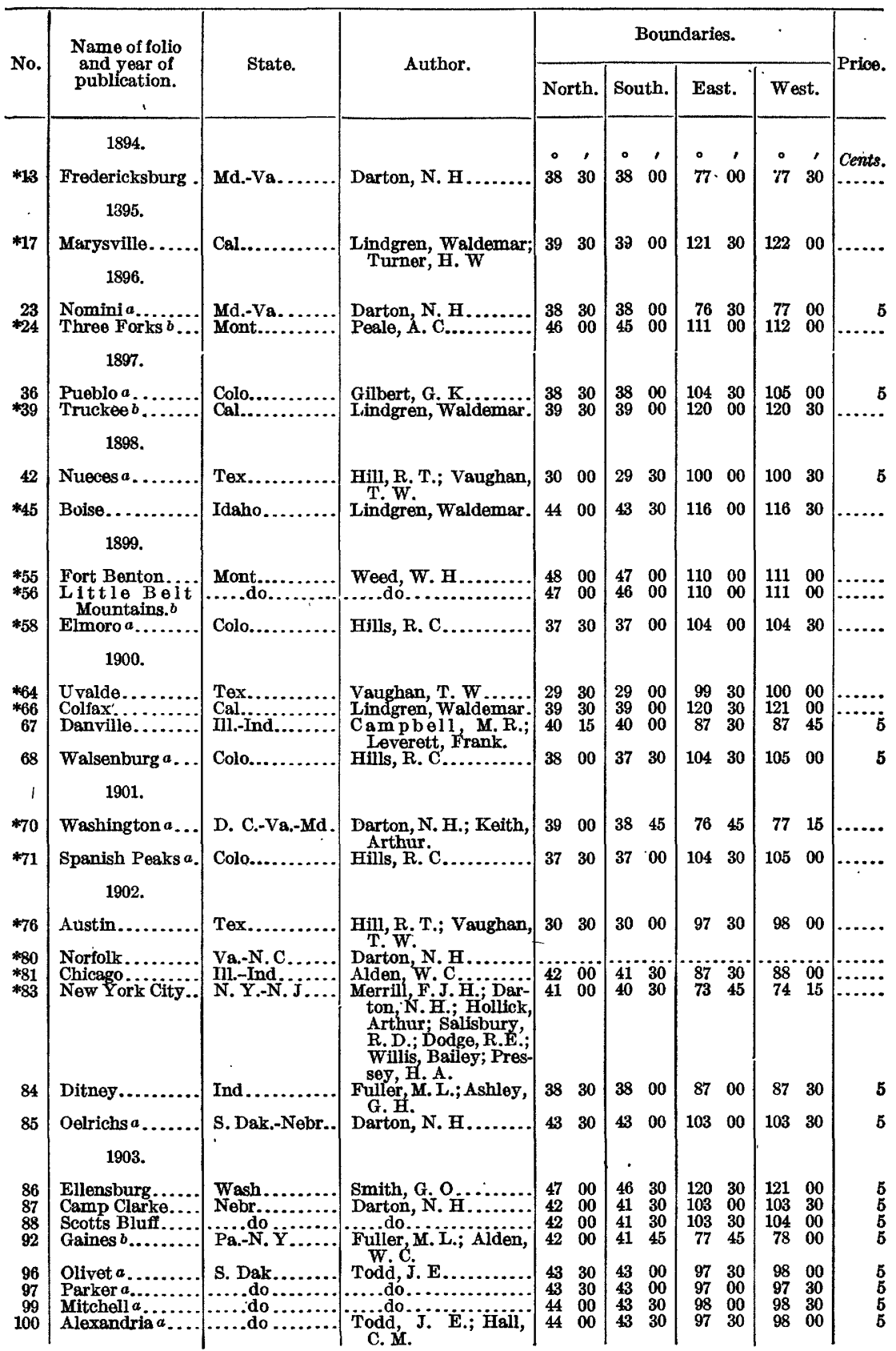

a Includes map showing artesian or other ground-water conditions.

$b$ Information on mineral water only. 
Geologic folios-Continued.

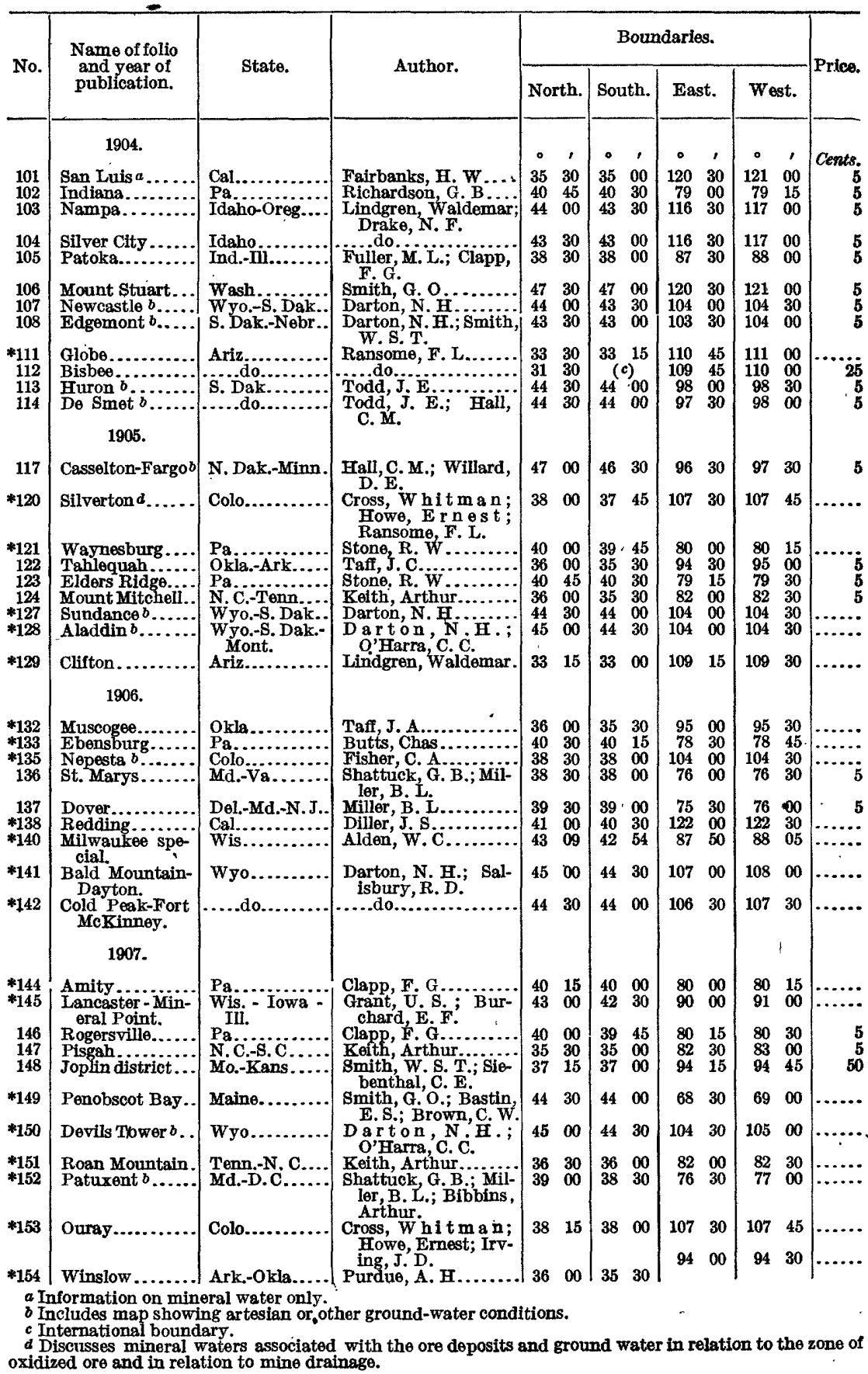


Geologic folios-Continued.

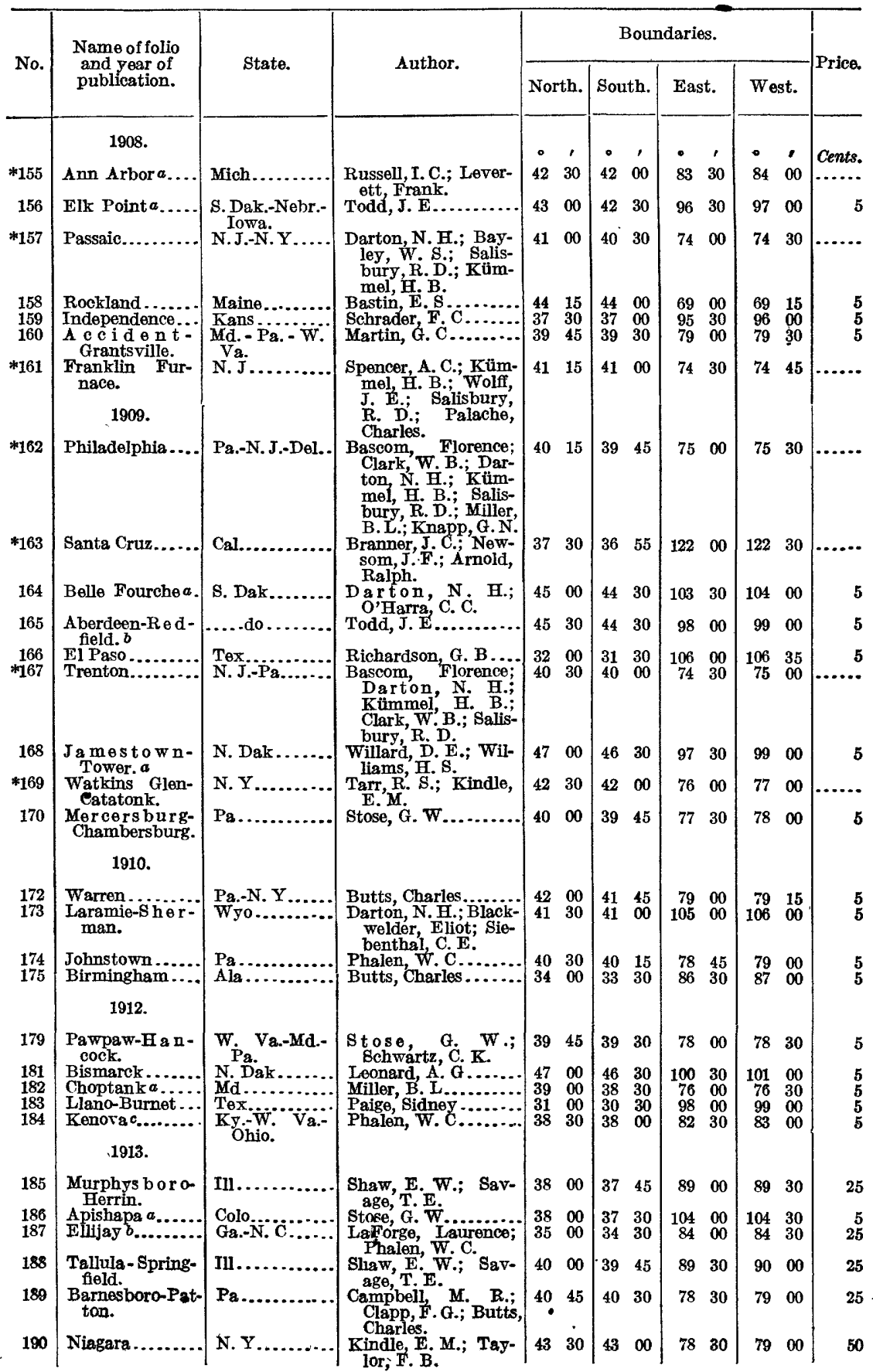

- Includes map showing artesian or other ground-water conditions.

$b$ Information on mineral water only.

$c$ Contains no discussion of ground water but a section and data regarding oil and gas wells which give information as to deptll at which salt water is found. 
Geologic folios-Continued.

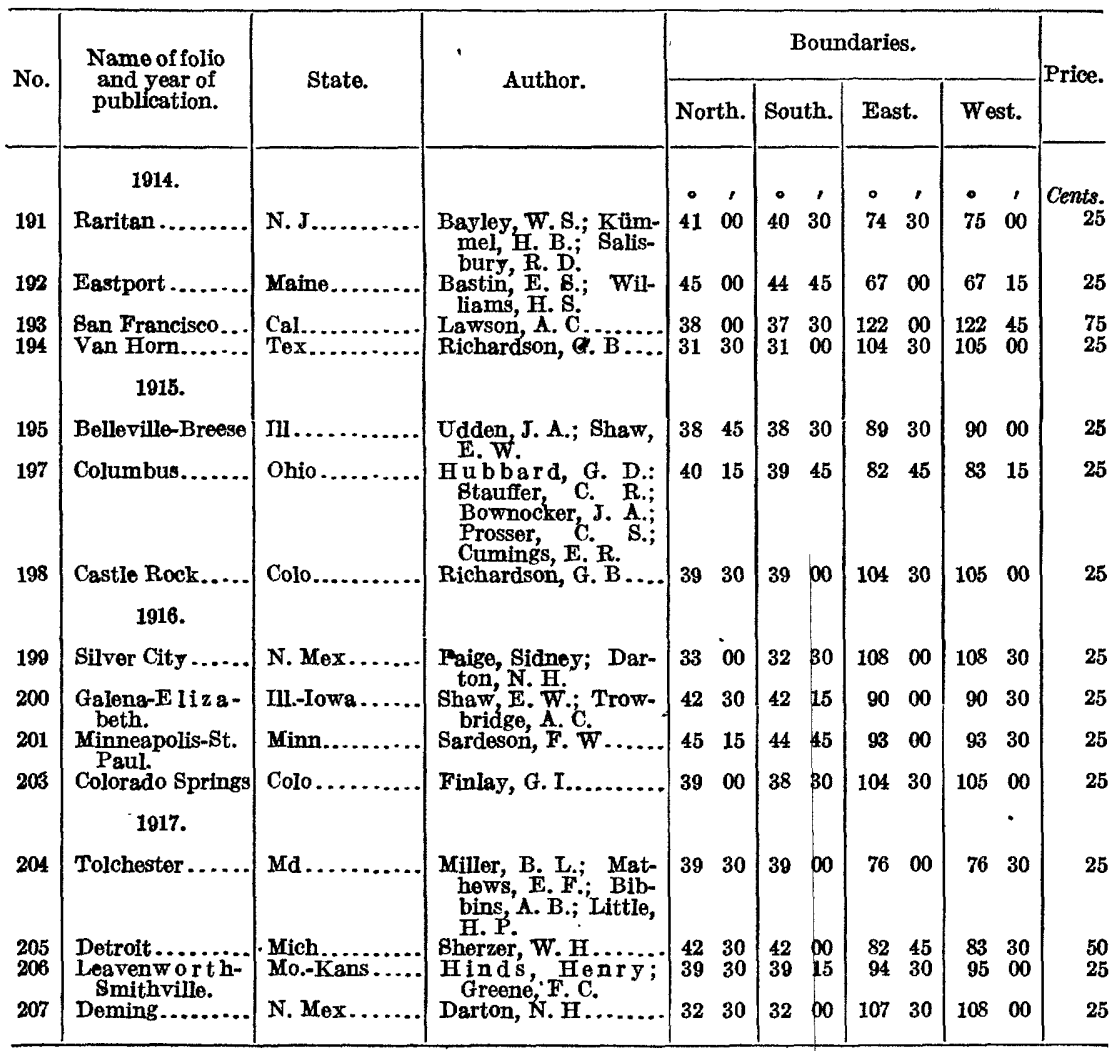

COOPERATIVE REPORTS NOT PUBLISHED BY THE UNITED STATES GEOLOGICAL SURVEY.

(S 1) Report of a reconnaissance in Trans-Pecos Texas, north of the Texas \& Pacific Railway, by G. B. Richardson. Texas Univ. Min. Survey Bull. 9; Texas Univ. Bull. 23, Austin, Tex., 1904.119 pp., 11 pls.

Describes the topography, geology, mineral resources, and ground waters of Toyah, Salt, and Huoco basins, Gypsum Plain, Guadalupe-Delaware Mountains, Diablo Plateau, and Rio Grande Valley. Contains a table of well data, well sections, and water analyses, and includes a map showing the geology and the locations of wells and springs.

(S 2) The underground water resources of Alabama, by E. A. Smith. Alabama Geol. Survey, Montgomery, Ala., 1907. 388 pp., 30 pls.

Describes the geography, geology, and climate of the State. Contains a general discussion in regard to the occurrence, circulation, and recovery of ground water and in regard to artesian wells. Gives detailed descriptions of wells, mineral waters, and ground-water conditions, with discussions of artesian prospects, by provinces, counties, and vicinities. Discusses the chemistry and classification of the waters from the various horizons and contains water analyses. Includes maps showing the geology and the areas of artesian flow.

(S3) Water resources of the East St. Louis district, by Isaiah Bowman and C. A. Reeds. Illinois State Geol. Survey Bull. 5, Urbana, Ill., 1907. 128 pp., 4 pls.

Discusses the economic features of the district as a manufacturing site and describes the topography and geology and the water supplies from springs, cisterns, streams, lakes, reservoirs, and wells. Discusses underground drainage, fluctuations of the water table, water resources of the Karst, contamination of Karst water, unusual phenomena associated with springs and wells, and artesian conditions. Contains well sections and mineral and sanitary analyses of water. The water resources of a part of this area are also described in U. S. Geological Survey Bulletin 438. 
(S 4) A preliminary report on the underground waters of Georgia, by S. W. McCallie. Georgia Geol. Survey Bull. 15, Atlanta, Ga., 1908. 370 pp., 30 pls.

Describes the physiography and geology of the State and discusses briefly precipitation, evaporation, and absorption by the soil. Describes in general the waters of the various geological formations, and in more detail the ground-water conditions in each county in the Coastal Plain, Crystalline area, and Paloozoic area. Contains numerous analyses of ground waters and discussions of their quality. Includes a chapter entitled "Experiment relating to problems of well contamination at Quitman" and one entitled "Blowing springs and wells of Georgia." Includes a table of data regarding public water supplies and a table of miscellaneous spring records. Also includes a geologic map of Georgia.

(S 5) The Coastal Plain of North Carolina, by W. B. Clark, B. L. Miller, L. W. Stephenson, B. L. Johnson, and H. N. Parker. North Carolina Geol. and Econ. Survey, vol. 3, Raleigh, N. C., 1912. 552 pp., 42 pls.

Consists of two parts bound in one volume. Part I describes the physiography and geology; Part II describes the water resources both surface and underground. Part II opens with a bibliography and a general discussion of the ground-water conditions but consists chiefly of detailed descriptions, by counties, of the topography, geology, ground-water conditions, and artesian prospects, with tables giving well data. These county descriptions are followed by a discussion of the quality of the water, by counties, and tables of analyses. The volume contains maps of the Coastal Plain of North Carolina showing the distribution of the surficial deposits and the underlying formations, the position of the basement rocks, and the areas in which potable water can be obtained from the various formations.

(S 6) Subterranean storage of flood waters by artificial methods in San Bernardino Valley, Cal., by C. H. Lee. Conservation Commission of California Rept. for 1912, Sacramento, Cal., 1912. . pp. 335-400, 3 pls.

Gives history and methods of water spreading and amount of water spread along Santa Ana River and other streams. Gives data in regard to precipitation, run-off, and stream percolation in the San Bernardino Basin. Describes the ground-water reservoir in this basin, giving data on fluctuations of the water table and the artesian head. Discusses ground-water fluctuations in other basins in Southern California and draws conclusions as to the effect of water spreading. Includes a map showing the original area of artesian flow in the San Bernardino Basin and the areas in 1904 and 1912, also locations of wells and of lands used for spreading water.

(S 7) Ground-water resources of Indian Wells Valley, Cal., by C. H. Lee. Conservation Commission of California Rept. for 1912, Sacramento, Cal., 1912. pp. $401-429,5$ pls.

A brief report covering an arid valley (also known as Salt Wells Valley) that lies mostly in northeastern Kern County but extends into Inyo and San Barnardino counties and comprises a part of the area included in Tps. 25-27, Rs. 38-40. Gives information in regard to precipitation, stream flow, evaporation of ground water, and water supply available by pumping from wells.

(S 8) The underground water resources of the Coastal Plain province of Virginia, by Samuel Sanford. Virginia Geol. Survey Bull. 5, Charlottesville, Va:, 1913. 361 pp., 1 pl.

Describes and ciscusses the topography, geology, origin, occurrence, and emergence of ground water, types of springs and their pollution, artesian conditions, cisterns, collecting tunnels, types of wells, magnetic wells, and freshening of deep water. Gives detailed information on wells and ground-water conditions by counties and contains tables of well data and of analyses of spring and well waters. Includes a map showing the areas of artesian flow and the quality of the ground waters.

(S 9) The underground and surface water supplies of Wisconsin, by Samuel Weidmen and A. R. Schultz. Wisconsin Geol. and Nat. Hist. Survey Bull. 35, Madison, 'Wis., 1915. 664 pp., 5 pls.

Describes the geography and geology of the State and the conditions controlling local ground waters and artesian waters. Describes the flowing wells and artesian prospects, the springs and mineral waters, the uses of water supplies, and the quality of water from various geologic sources and from the rivers and lakes of the State. Gives detailed descriptions by counties with well sections and water analyses. Includes maps showing the geology, the surface of the pre-Cambrian rocks, the head of artesian water, and the areas of soft and hard water. 
(S 10) Memorandum on the geology of the ground waters of the Island of Antigua, B. W. I., by T. W. Vaughan. Imperial Dept. Agr. for the West Indies, West Indian Bull., vol. 14, No. 4, 1915. pp. 276-280.

Outlines the physiography and geology of Antigua and their bearing on ground-water conditions. Discusses the prospects of obtaining artesian and other ground waters; also discusses the quality of the ground waters, especially with reference to salt. Includes a sketch map of the island showing the geology. Reference is made to the work of H. A. Tempany, chemist and superintendent of agriculture for the Leeward Islands, who published two papers on the same subject, containing some detailed spring and well data and a number of analyses of ground water. The papers by Tempany are as follows: "The water supply of Antigua" (West Indian Bull., vol. 12, No. 4) and "The ground waters of Antigua" (West Indian Bull., vol. 14, No. 4).

(S 11) Reports on condition of water supply at Savannah, Ga. Mayor of Savannah Ann. Rept., 1915.

Contains the following papers submitted by the United States Geological Survey:

Preliminary report on Savannah Water Supply, by L. W. Stephenson and R. B. Dole. Pp. 1-14.

The water supply of Savannah, Ga., by R. B. Dole. Pp. 15-89.

These papers discuss the yield and head of the artesian wells of Savannah, the consumption of water, the sanitary and chemical quality of the water, and the cost of operation. They givo the results of fluorescein tests and several analyses of surface and ground waters. They conclude with recommendations for future developments.

(S 12) Report of the Water Commission of the Territory of Hawaii to the Governor of Hawaii, by G. K. Larrison, A. G. Smith, and T. F. Sedgwick. Honolulu, 1917. 53 pp., map, diagrams, and tables.

Discusses and gives data regarding artesian water on the Island of Oahu, the relation of precipitation to artesian head, the discharge of flowing and pumped wells, the interference of wells, the progress of artesian well boring, and waste of water from artesian wells. Includes a proposed law relating to the use of water from artesian wells. The data were collected by $R$. C. Rice and R. D. Klise, of the United States Geological Survey, under the direction of T. F. Sedgwick.

\section{MISCELLANEOUS SPRING RECORDS.}

Miscellaneous records of the discharge of springs in papers not elsewhere listed.

\begin{tabular}{|c|c|c|c|c|c|c|}
\hline $\begin{array}{c}\text { Water- } \\
\text { Sup- } \\
\text { ply } \\
\text { Paper. }\end{array}$ & $\begin{array}{l}\text { Pages on } \\
\text { which } \\
\text { data are } \\
\text { given. }\end{array}$ & $\begin{array}{c}\text { Date of meas- } \\
\text { urement. }\end{array}$ & Name. & State. & Location. & $\begin{array}{l}\text { Dis- } \\
\text { charge. }\end{array}$ \\
\hline 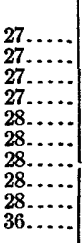 & $\begin{array}{r}45 \\
45 \\
45 \\
45 \\
130 \\
130 \\
130 \\
130 \\
130 \\
147\end{array}$ & $\begin{array}{l}\text { Dec. } 20,1898 \\
\text { Dec. } 21,1898 \\
\text { Dec. 22, } 1898 \\
\text { Dec. 23, } 1898 \\
\text { Mar. 26, } 1898 \\
\text { May 3, } 1898 \\
\text { De.do.... } 23,1898 \\
\text { Dec. 24, } 1898 \\
\text { May 30, } 1899\end{array}$ & 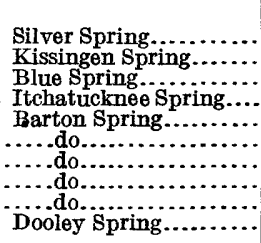 & 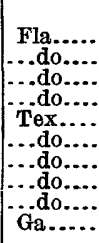 & 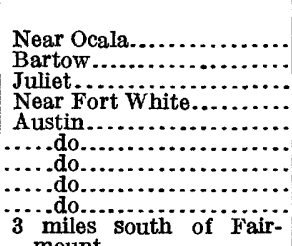 & $\begin{array}{c}\text { Sec.ft. } \\
822 \\
31 \\
778 \\
403 \\
20 \\
30 \\
31 \\
19 \\
19 \\
.92\end{array}$ \\
\hline $\begin{array}{l}36 . . \\
36 . .\end{array}$ & $\begin{array}{l}147 \\
147\end{array}$ & -...do.. & $\begin{array}{l}\text { Drove Mill Spring....... } \\
\text { Baker's mill spring.... }\end{array}$ & (..do.. & 5 miles south of Fair- & $\begin{array}{r}16.2 \\
3.9\end{array}$ \\
\hline $\begin{array}{l}36 \ldots \\
36 \ldots \ldots \\
36 \ldots \ldots\end{array}$ & $\begin{array}{l}147 \\
147 \\
147\end{array}$ & $\begin{array}{l}\text { June } 24,1899 \\
\ldots \ldots . \text { do.......... } \\
\ldots . \text { do } \ldots \ldots \ldots\end{array}$ & $\begin{array}{l}\text { Colina Spring............ } \\
\text { Arnold Spring .......... } \\
\text { J. R. Byrd's spring..... }\end{array}$ & $\begin{array}{l}\text {..do.. } \\
\text {..do.. } \\
\ldots \text { do.. }\end{array}$ & $\begin{array}{l}\text { Near Colina } \\
1 \text { mile south of Golina...... } \\
2 \frac{1}{2} \text { miles northwest of Fair- }\end{array}$ & $\begin{array}{l}.28 \\
.38 \\
.47\end{array}$ \\
\hline $\begin{array}{l}36 \ldots \\
36 \ldots \ldots \\
36 \ldots \ldots \\
36 \ldots \ldots \\
36 \ldots \\
6\end{array}$ & $\begin{array}{l}147 \\
147 \\
147 \\
147 \\
147\end{array}$ & 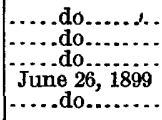 & $\begin{array}{l}\text { R. W. Lander's spring. } \\
\text { J. A. Johnson's spring.. } \\
\text { Oakhill Church Spring. } \\
\text { Hayse Spring........... } \\
\text { Cedar Spring........... }\end{array}$ & \begin{tabular}{l}
.. do. \\
\hdashline do. \\
\hdashline do. \\
\hdashline do. \\
\hdashline do. \\
\hdashline .do.
\end{tabular} & 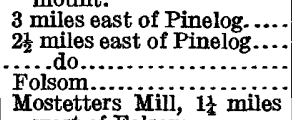 & $\begin{array}{r}4.27 \\
.48 \\
1.85 \\
.35 \\
4.45\end{array}$ \\
\hline 36. & 147 & . & Trimble Spring. & $\ldots d$ & 2 miles north of Adairs- & 2.35 \\
\hline 36. & 147 & ...do. & Gardners Spring. & ...do.. & $\begin{array}{l}5 \text { miles north of Adairs- } \\
\text { ville. }\end{array}$ & .41 \\
\hline
\end{tabular}


Miscellaneous records of the discharge of springs in papers not elsewhere listed-Contd.

\begin{tabular}{|c|c|c|c|c|c|c|}
\hline $\begin{array}{c}\text { Water- } \\
\text { Sup- } \\
\text { ply } \\
\text { Paper. }\end{array}$ & $\begin{array}{l}\text { Pages on } \\
\text { which } \\
\text { data are } \\
\text { given. }\end{array}$ & $\begin{array}{l}\text { Date of meas- } \\
\text { urement. }\end{array}$ & Name. & State. & Location. & $\begin{array}{c}\text { Dis- } \\
\text { charge. }\end{array}$ \\
\hline 36. & 147 & June 26,1899 & Blackwood Sp & & 5 mile & $\underset{1}{S e c .-f t .}$ \\
\hline 37. & 277 & June 30, 1899 & Las Moras Spri & $\mathbf{T}$ & $\begin{array}{l}\text { Fort Clark, near Brackett- } \\
\text { ville, and } 10 \text { miles from } \\
\text { Spofford. }\end{array}$ & 60 \\
\hline 37. & $\begin{array}{l}277 \\
277\end{array}$ & $\begin{array}{l}\text { Dec. 24, } 1895 \\
\text { June 29, } 1899\end{array}$ & San Felipe Spr & & Below & $\begin{array}{l}21 \\
84\end{array}$ \\
\hline $\begin{array}{l}37 . \\
39 .\end{array}$ & $\begin{array}{l}277 \\
434\end{array}$ & Aug. 21,1899 & $\begin{array}{l}\text { San Madre Ditch....... } \\
\text { Pacoima submerged }\end{array}$ & & 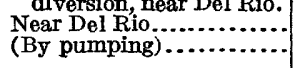 & ${ }^{29} .24$ \\
\hline 39. & 440 & June 13, 1899 & wooden spring & Nel & Sec. 25, T. 35 N., R. 29 W. & 2.14 \\
\hline 85. & 126 & Aug. 4, 1902 & Spring.......... & $\mathrm{Ne}$ & $\begin{array}{l}\frac{1}{2} \text { mile north of Heitman's } \\
\text { house. }\end{array}$ & .74 \\
\hline $\begin{array}{l}85 \ldots \\
85 \ldots \\
85 \ldots \\
85 \ldots \\
85 \ldots \\
85 . \\
85 \ldots\end{array}$ & $\begin{array}{l}126 \\
126 \\
126 \\
126 \\
126 \\
126 \\
216\end{array}$ & $\begin{array}{l}\text { Sept. 8, } 1902 \\
\text { Aug. 4, } 1902 \\
\text { Sept. 8, } 1902 \\
\text { Aug. 4, } 1902 \\
\text { Sept. 8, } 1902 \\
\text { June } 10 \text { and }\end{array}$ & 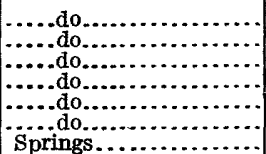 & 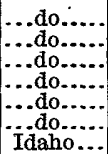 & 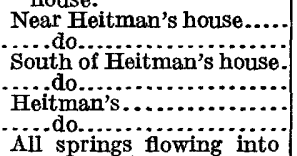 & $\begin{array}{r}.35 \\
.27 \\
1.08 \\
1.06 \\
2.39 \\
2.35 \\
112\end{array}$ \\
\hline & 293 & $\begin{array}{l}11,1902 . \\
\text { July } 20,1903\end{array}$ & Big Springs.. & 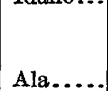 & $\begin{array}{l}\text { Snake River, between } \\
\text { Shoshone Falls and the } \\
\text { Cedars. } \\
\text { Tuscumbia............................. }\end{array}$ & Now \\
\hline 127. & 120 & Apr. 19, 1904 & …. do....... & & $\begin{array}{l}\frac{1}{2} \text { milefrom left bank Flint } \\
\text { River, about } 4 \text { miles } \\
\text { down stream from Al- } \\
\text { bany. }\end{array}$ & 135 \\
\hline $127 \ldots$. & $\begin{array}{l}120 \\
120\end{array}$ & $\begin{array}{l}\text { Sept. 23, } 1904 \\
\text { Nov. 16, } 1904\end{array}$ & 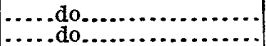 & & 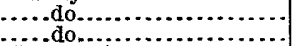 & $\begin{array}{l}44 \\
26.4\end{array}$ \\
\hline & 175 & Jan. 21, 1904 & Cave Sprin & $\because \boldsymbol{u}$ & Cave & $\begin{array}{r}20.4 \\
5.3\end{array}$ \\
\hline & 192 & Sept. 11, 1904 & Giant Sprir & Mor & Gre & 202 \\
\hline & $\begin{array}{l}358 \\
358\end{array}$ & Aug. 31, 1904 & $\begin{array}{l}\text { Heitman's sprin } \\
\text { Monfrena Spring }\end{array}$ & Nev & Mouth of & 1.2 \\
\hline 133. & $\begin{array}{l}000 \\
364\end{array}$ & Oct. 4,1904 & Big Spring.... & Utah.... & $\begin{array}{l}\text { Tributary to Rock Greek, } \\
\text { in Duchesne } R \text { i ver }\end{array}$ & $\begin{array}{l}1.2 \\
7.4\end{array}$ \\
\hline 135. & 271 & Aug. 10, 1904 & Hawley Springs. & Idah & $\frac{1}{2}$ mile below Heise post & .8 \\
\hline 135. & 272 & Aug. 17, 1904 & Thurman Sprin & -...00 & North Fork of $\mathrm{S} \mathrm{n}$ & .2 \\
\hline 135. & 272 & ....do.. & West B ald Cabin & at & mive & .2 \\
\hline 135 . & 272 & .....do........ & East Bald Cabin & & .... do.. & 1.5 \\
\hline 135 & 272 & .....do. & Green Springs. & $\cdots$ & .....do. & .6 \\
\hline $\begin{array}{l}135 . \\
135 .\end{array}$ & $\begin{array}{l}272 \\
272\end{array}$ & $\begin{array}{l}\text { Aug. 18, } 1904 \\
\ldots \text { do............ }\end{array}$ & $\begin{array}{l}\text { Blue Springs... } \\
\text { Grizzly Spring. }\end{array}$ & & $\begin{array}{l}\text {......do } \\
\ldots . . . \text { do }\end{array}$ & $\begin{array}{l}3.6 \\
5.3\end{array}$ \\
\hline $135 \ldots$ & 272 & Aug. 20, 1904 & Caldwell Spring $\mathrm{N}$ & & .....do... & .5 \\
\hline $135 \ldots$. & $\begin{array}{l}272 \\
272\end{array}$ & Aug. 22, 1904 & $\begin{array}{l}\text { Caldwell Spring Nc } \\
\text { Sorenson Spring... }\end{array}$ & $\ldots d$ & .....do.... & .3 \\
\hline $135 \ldots$ & 273 & Aug. 23, 1904 & Bear or Little Spris & $\cdots$...dc & $\begin{array}{l}\text { East bank of Henry Fork } \\
\text { of North Fork of Snake } \\
\text { River. }\end{array}$ & .0 \\
\hline 135. & 273 & ......do.. & Thompson Spring & do & .....do.. & 5 \\
\hline & 273 & Audo........ 1904 & $\begin{array}{l}\text { Gar } \\
\text { Wh }\end{array}$ & $\ldots d c$ & Tributori & $\stackrel{99}{7}$ \\
\hline & 273 & 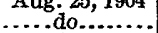 & Rock & -8 & .....do... & \\
\hline & 273 & ....do......... & d Springs & & ....do... & 1.2 \\
\hline & 273 & .....do.......... & Big S & $\cdots$ ad & .....do.... & 1.2 \\
\hline & $\begin{array}{r}268 \\
98\end{array}$ & Apr. 26,1905 & Blue Spring... & Ga. & 4 miles from Albany on & $69^{\circ}$ \\
\hline 168. & 101 & Mar. 10, 1905 & Warm Springs & $d a_{2}$ & $\frac{1}{2}$ milefrom Warm Springs & 3.2 \\
\hline 68. & 101 & Apr. 20, 1905 & do & $\cdots$ & $\ldots$. & 1.47 \\
\hline & 101 & & & & Nor Ria Pin & 1.48 \\
\hline $177 . .$. & $\begin{array}{r}85 \\
245\end{array}$ & $\begin{array}{l}\text { Dec. 4, } \\
\text { Aug. 29, } 1905\end{array}$ & $\begin{array}{l}\text { Fish Springs...... } \\
\text { Little Shasta Spri }\end{array}$ & $\begin{array}{l}\text { Cal. } \\
\text {..do }\end{array}$ & $\begin{array}{l}\text { Near Big P1 } \\
\text { Little Shast }\end{array}$ & 20 \\
\hline & 241 & Mar. 10,1905 & Warm Springs.... & Ga.. & $\frac{1}{2}$ mile from Warm springs & 3.2 \\
\hline $197 . \ldots$ & $\begin{array}{l}241 \\
241\end{array}$ & 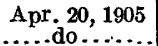 & warm & Ga... & & $\begin{array}{l}1.47 \\
1.48\end{array}$ \\
\hline $197 \ldots$ & $\begin{array}{r}302 \\
92\end{array}$ & Jan. 21,1904 & $\begin{array}{l}\text { Cave Spring } \\
\text { A. Bayter's dug weil }\end{array}$ & Ütah.... & Cave Spring..... & $\begin{array}{l}5.3 \\
.11\end{array}$ \\
\hline $212, \ldots$. & 92 & July 12,1906 & N. Green's spring... & ..do.. & Nẹar fence No. $48 . . . . . . .$. & .25 \\
\hline
\end{tabular}


Miscellaneous records of the discharge of springs in papers not elsewhere listed-Contd.

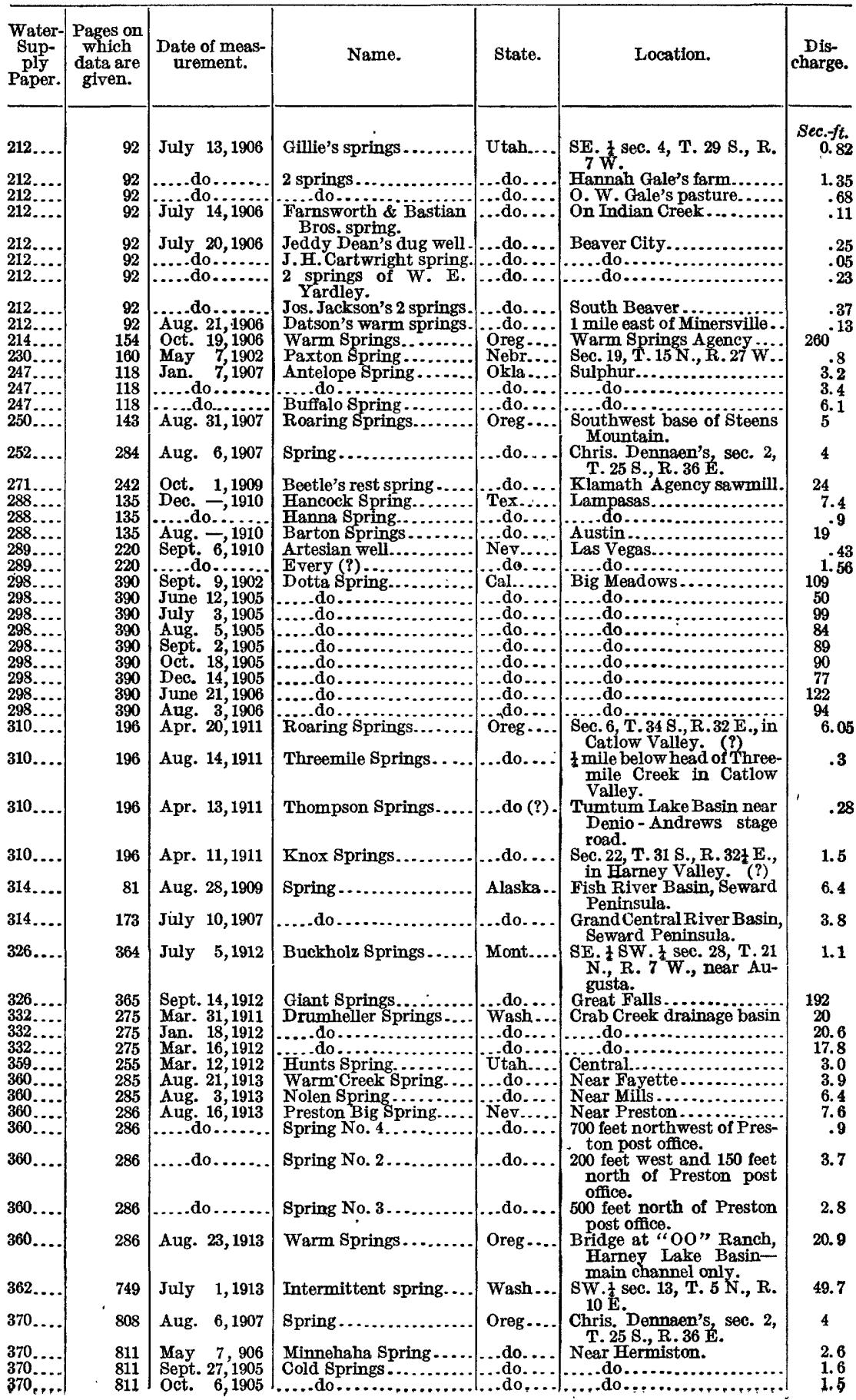


Miscellaneous records of the discharge of springs in papers not elsewhere listed-Contd.

\begin{tabular}{|c|c|c|c|c|c|c|}
\hline $\begin{array}{l}\text { Water } \\
\text { Sup- } \\
\text { ply } \\
\text { Paper. }\end{array}$ & $\begin{array}{l}\text { Pages on } \\
\text { which } \\
\text { data are } \\
\text { given. }\end{array}$ & $\begin{array}{l}\text { Date of meas- } \\
\text { urement. }\end{array}$ & Name. & State. & Location. & $\begin{array}{l}\text { Dis- } \\
\text { charge. }\end{array}$ \\
\hline $\begin{array}{l}370 \ldots . . \\
370 \ldots \\
370 \ldots\end{array}$ & $\begin{array}{l}811 \\
817 \\
818\end{array}$ & $\begin{array}{l}\text { Oct. 2,1905 } \\
\text { Sept. 20,1908 } \\
\text { Aug: } 7,1907\end{array}$ & $\begin{array}{l}\text { Gillette Spring } \\
\text { Anna Creek S } \\
\text { Beetles Rest S }\end{array}$ & $\begin{array}{l}\text { Oreg.... } \\
\ldots \text { do.... } \\
\ldots . \text { do .... }\end{array}$ & $\begin{array}{l}\text { Near Echo... } \\
\text { Arants...... } \\
\frac{1}{2} \text { mile north }\end{array}$ & $\begin{array}{l}\text { Sec.-ft. } \\
2.7 \\
2.7 \\
25\end{array}$ \\
\hline $\begin{array}{l}370 \ldots \\
370 \ldots \\
370 \ldots \\
370 \ldots\end{array}$ & $\begin{array}{l}818 \\
818 \\
818 \\
818\end{array}$ & $\begin{array}{lr}\text { Mar. } & 23,1908 \\
\text { Apr. } & 27,1908 \\
\text { Oct. } & 1,1909 \\
\text { May } & 8,1910\end{array}$ & 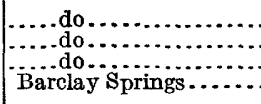 & $\begin{array}{l}\ldots d \\
\cdots d d \\
\cdots d \\
\cdots d\end{array}$ & Üper Klamath Lake & $\begin{array}{l}26 \\
25 \\
24 \\
1.7\end{array}$ \\
\hline $\begin{array}{l}370 . \\
370 .\end{array}$ & $\begin{array}{l}820 \\
821\end{array}$ & $\begin{array}{l}\text { May } 16,1908 \\
\text { Aug. } 31,1907\end{array}$ & $\begin{array}{l}\text { Olene Springs. } \\
\text { Roaring Spring }\end{array}$ & $\ldots \mathrm{dc}$ & Near Olene & $\frac{2.9}{5}$ \\
\hline 389 & 191 & Apr. 26,1914 & Toquerville Sprir & Utah & $\begin{array}{l}\text { East side of Ash Creek, } \\
\text { just above Toquerville. }\end{array}$ & 1 \\
\hline 389 & 191 & Feb. 12, 1914 & Hunts Sprir & $\ldots d$ & $\begin{array}{l}\text { Hunt's ranch, } 1 \text { mile south- } \\
\text { east of Central. }\end{array}$ & 3.2 \\
\hline & $\begin{array}{l}191 \\
191\end{array}$ & $\begin{array}{l}\text { Apr. } 27,1914 \\
\text { Dec. } 11,1913\end{array}$ & stone Cabin sp & ...do. & 7 milesnorthwesto & $\begin{array}{l}3 \\
2\end{array}$ \\
\hline & 191 & May 23, 1914 & 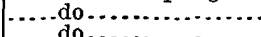 & $\ldots d$ & $\ldots$. do & 1.8 \\
\hline & $\begin{array}{l}191 \\
191\end{array}$ & $\begin{array}{l}\text { July } 17,1914 \\
\text { Aug. } 7,1914\end{array}$ & $\cdots \cdots$ & $\cdots$ & $\cdots . . . d$ & $\begin{array}{l}1.8 \\
1.8\end{array}$ \\
\hline & 191 & Aug. 26,1914 & Ash Moa & $\cdots d$ & ...do.... & 1.8 \\
\hline & $\begin{array}{l}192 \\
297\end{array}$ & June 21,1914 & Mollen Springs. & $\ddot{\mathrm{U}} \mathrm{a}$ & 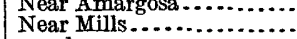 & $\begin{array}{l}3.4 \\
5.6\end{array}$ \\
\hline & 297 & 23,1914 & $\cdots \cdots$ & $\ldots d$ & .... do do...... & 7. \\
\hline & $\begin{array}{l}297 \\
297\end{array}$ & Aug. 28, 1914 & Mollen springs. & $\cdots$ & $\begin{array}{l}\text {.....do } \\
\cdots . . . \text { do }\end{array}$ & $\begin{array}{l}4.7 \\
6.8\end{array}$ \\
\hline & 297 & Aug. 28, 1914 & $\because \ldots c$ & $\because u^{d}$ & Presto...... & 7.6 \\
\hline & 297 & 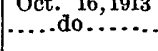 & Spring ....................... & $\mathrm{Ne}$ & 200 feet northwest of Pres- & $\begin{array}{l}7.6 \\
3.6\end{array}$ \\
\hline & $\begin{array}{l}297 \\
298\end{array}$ & Juily 14,1914 & Parsnip Sprin & Oreg. & $\begin{array}{l}\text { Near Gee's at Preston.... } \\
\text { At mouth of Camas Creek. }\end{array}$ & $\begin{array}{l}2 \\
1.3\end{array}$ \\
\hline & 328 & Aug. 24, & Big & $\ldots d$ & $\begin{array}{l}\text { Fort Klamath-Crescent, } \\
\text { road crossing. }\end{array}$ & 61.4 \\
\hline $\begin{array}{l}391 . \\
391 .\end{array}$ & $\begin{array}{l}328 \\
328\end{array}$ & $\begin{array}{l}\text { Aug. } 12,1914 \\
\ldots \ldots \text { do }\end{array}$ & & . . d & mile below road below & $\begin{array}{l}70.8 \\
62.5\end{array}$ \\
\hline 3 & 328 & Aug. 27, 1914 & Roger Spring & 1.940 & mile above mouth of & 5 \\
\hline & 241 & Mar. 24, 1914 & Warr & Ida & Near Henry ... & .6 \\
\hline & $\begin{array}{l}241 \\
241\end{array}$ & $\begin{array}{l}\text { Apr. } 17,1914 \\
\text { May } 2,1914\end{array}$ & $\ldots \ldots$ & $\ldots d$ & 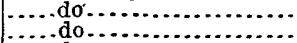 & $\begin{array}{l}1 \\
1.2\end{array}$ \\
\hline & 241 & June 26, 1914 & $\cdots$ & & ..... do ................ & 1.4 \\
\hline & $\begin{array}{l}241 \\
173\end{array}$ & $\begin{array}{l}\text { Aug. 6, 1914 } \\
\text { Aug. 15, } 1914\end{array}$ & Sprin & Ore & $\begin{array}{l}\text { Tributary to Deschutes } \\
\text { River between Spring }\end{array}$ & $\begin{array}{r}1.1 \\
13.5\end{array}$ \\
\hline 394 & 173 & Aug. 14, 1914 & ... & $\ldots$. d & $\begin{array}{l}\text { River and West's bridge. } \\
\text { Tributary to Deschutes } \\
\text { River between West's } \\
\text { bridge and Benham } \\
\text { Falls. }\end{array}$ & 28.4 \\
\hline & $\begin{array}{l}174 \\
174\end{array}$ & $\begin{array}{l}\text { July } 3,1914 \\
\text { Sept. 16,1914 }\end{array}$ & 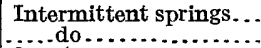 & $\begin{array}{l}\text { Wash... } \\
\ldots \text {... do... }\end{array}$ & 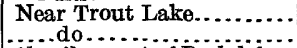 & $\begin{array}{ll}32.6 \\
0\end{array}$ \\
\hline & 174 & Mar. 18, 1914 & $3 \mathrm{spr}$ & & $1 \frac{1}{2}$ miles west of Parkdale. & 13.3 \\
\hline
\end{tabular}




\section{INDEX.}

The following abbreviations are used in the index:

W, Water-supply paper (pp. 5-29).

A, Annual report (pp. 29-32).

MR, Report on mineral resources (p. 42).

M, Monograph (pp. 32-33).

P, Professional paper (pp. 33-34).

B, Bulletin (pp. 34-40).

GF, Geologic folio (pp. 43-45).

$\mathrm{S}$, Cooperative report not published by the United States Geological Survey (pp. 45-47).

Names of formations and geologic ages are entered as used in the reports indexed without revision to bring them into accord with current usage.

\section{A.}

Absorption areas for artesian water........................... A 5 c

Absorption of water, apparatus for measuring....................... 155

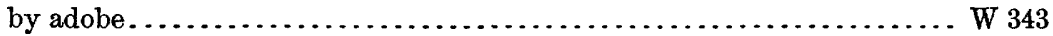

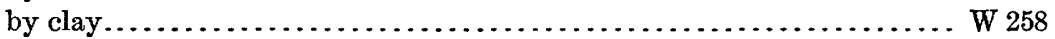

by conglomerate.................................... 258

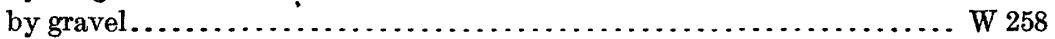

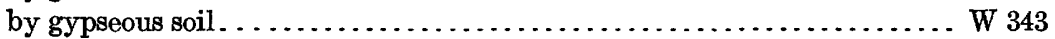

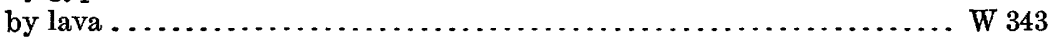

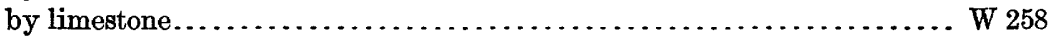

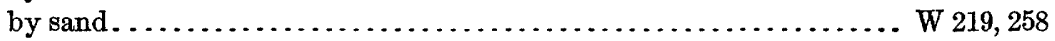

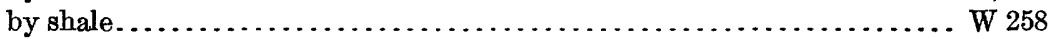

by sink holes. .............................. W $233 ;$ A 16 m f

by slate. . . . . . . . . . . . . . . . . 258

by soil............................................. $294 ;$ S 4

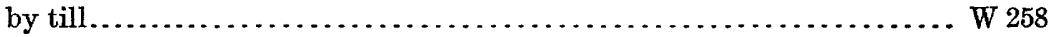

by valley fill....................................... 423

drainage wells in relation to............................. W 258

estimates of.................................. 219, 294, 423

from floods.................................. W 294, 380; S 6

from irrigation............................................. 294

from precipitation...................... 153, 155, 294, 380, $400 \mathrm{e}$ from streams................ W 153, 158, 199, 294, 345, $375 \mathrm{~d}, 380,400 \mathrm{e}, 423 ; \mathrm{S} 6$

in alkali flats................................................. 343

lysimeters for measuring ....................................... 155

measurement of . . .......................... 155, 294, 400 e, 423

principles of ..................W 114, 159,219, 257; A 14 пा a, 19 пr b; S 2,8

spreading of flood waters in relation to......................... S 6

See also Quantity of ground water.

Acidity of ground water. See under Analyses and Quality.

Adams, G. I., Economic geology of Iola quadrangle, Kans................. B 238

Patrick and Goshen Hole quadrangles, Wyo. and Nebr.............. W 70

Water supply of Ozark region in northern Arkansas................. W 110

Adaville formation, water in, in Wyoming....................... P 53

Africa, deep wells in ................................... W 257

Agriculture. See Dry farming and Irrigation. 
Air in soils, flow of

A $19 \pi \mathrm{r}$

ground water in relation to.

A 19 II b

Air lifts.

W 256,343

Alabama, areas, all of State............................ 102, 114; S 2

areas, Birmingham quadrangle.......................... GF 175

Marengo County ...................................... B 298

Mobile County ....................................... B 298

artesian water in. . . . . . . . . . . . . . . . . . . . 2

bibliography of ground water in...................W 114, 120, 149, 163

mineral water in.................... W 114; B 32; MR 1883-1916; GF 175

quality of ground water in..............W 102, 114; B 32; GF 175; S 2

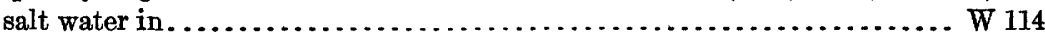

springs in, records for............................... W 98; B 32

temperature of ground water in............................... S 2

temperature of spring waters in............................ B 32

wells in, records for........................... W 57, 149; B 264, 298

Alachua clay, water in, in Florida................................. W 319

Alaska, bibliography of ground water in....................... 120, 163

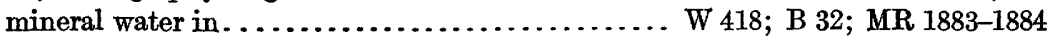

quality of ground water in . . . . . . . . . . . . . . . . . . . . . . . . . . . 418

springs in........................................ 314,418 ; B 32

temperature of ground water in...................... W $418 ;$ B 32

Alden, W. C., Chicago folio, Ill . .............................. GF 81

Gaines folio, Pa.-N. Y..................................... 92

Milwaukee special folio, Wis............................... GF 140

Algae in ground water......................... W 315, 338, 345 g; A 9 d

Algeria, artesian water in .................................... A 11 II c

bibliography of ground water in.................................. 163

irrigation with ground water in............................ A 11 II $\mathrm{c}$

Algonkian formations. See under Pre-Cambrian; also see specific formations.

Alkali as indicator of ground water................................ W 423

Alkali coefficient of water for irrigation. . . . . . . . . . . . . . $\ldots \ldots \ldots \ldots$ W 274, 335, 398

Alkali flats, water in .................................. W $422 ;$ B 530 r

Alkali in ground water. See Analyses; Quality.

Alkali in soil, water table in relation to....................W 320, 343, 422, 423

Alkalinity of ground water. See Ánalyses and Quality.

Alluvial fans. See Valley fill and Débris-filled basins.

Alluvium interbedded with lava, artesian water in.................... B 199

Alluvium, water in ....................................... W 257, 258

water in, in Antigua........................................ S 10

in Arkansas............................................... W 399

in California................ W 112, 140, 142, 219, 294, $345 \mathrm{~h}, 375 \mathrm{a}, 400 \mathrm{e}$

in Connecticut....................................W 374, 397

in Illinois.............................. A 17 II; GF 195; S 3

in Iowa. . . . . . . . . . . . . . . . . . . . . . . . . . 293

in Kansas................................... 153, 258, 273; GF 206

in Kentucky . . . . . . . . . . . . . . . . . . . . . . . . . . . . . . . W 233

in Michigan............................................. GF 205

in Minnesota................................. W 256; GF 201

in Mississippi............................................. W 159

in Missouri............................................ GF 206

in Nebraska.............................. $12,184,425$ b; GF 89

in New Mexico............................ W 140, 141, 158, 188, 422

in North Dakota. . .................................. GF 181 
Alluvium, water in, in Ohio................................. 91,259

in Pennsylvania............................................ 114

in Utah........................................... 157,217

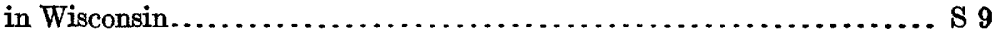

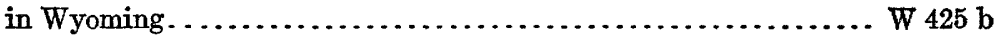

See also Valley fill, Gravel, and Sand.

Alum Bluff formation, water in, in Florida. . . . . . . . . . . . . . . . . . W 319

water in, in Georgia. ................................... W 341

Aluminum in ground water.................... W 273, 338; B 47; MR 1911

Ammon, on flow of air through soils.......................... A 19 II b

Ammonia in ground water........................ W 240, 256; MR 1911 In $n$

Amsden formation, water in, in Wyoming.......................... P 53

Anacacho formation, water in, in Texas. .................... A $18 \mathrm{~m} \mathrm{~b}$; GF 64

Analyses, mechanical, of water-bearing material...... W 67, 104, 136; A 19 m b; P 44

Analyses of ground water, bacteriologic................. W 193, 256, 259; S 11

bacteriologic, methods of . . . . . . . . . . . . . . . . . . . . . . 193

bibliography of . ................... B 330,491, 606, 616; MR $1913 \mathrm{~m}$ h, 1916

in Alabama.............................. W 102; B 32; GF 175; S 2

in Alaska..................................................... 418

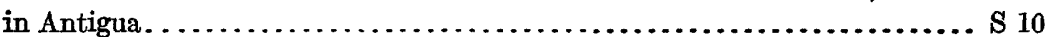

in Arizona..................... W 104, 136, 320, 364, $375 \mathrm{~b}$; B 32; GF 129

in Arkansas. . . . . . . . . . W 102, 145, 364, 399; P 45; B 32, 330, 491, 606, 616

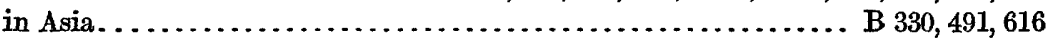

in Australia........................................ B 330, 491, 616

in Austria-Hungary....................... B 330, 491, 616; MR 1916

in British Columbia. ............................... B $330,491,616$

in California.... W 58, 59, 116, 142, 255, 278, 338, 364, 398; B 32, 330, $491,529,540 \mathrm{n}$,

$606,616,653 ;$ MR 1916

in Canada.................................. B $330,491,616$

in Colorado.... W 240, 364; A 17 II f; M 27; P 32, 52; B 32, 330, 491, 529, 606, 616;

GF 135, 153, 203; MR 1916

in Columbia.................................... B 330, 491, 616

in Connecticut................... W 102, 110, 232, 374, 397; P 44; B 32

in District of Columbia. . . . . . . . . . . . . . . . . . . . . . . . W $364 ;$ B 32,138

in England................................. B 330, 491, 616; MR 1916

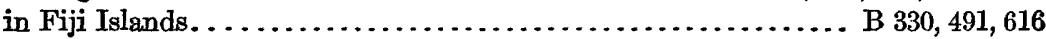

in Florida................................. W 102, 319, 364; B 32

in France............................... 330, 491, 616; MR 1916

in Georgia. ......................... W 102, 160, 341; B 32, 138; S 4, 11

in Germany. ...................... B 330, 491, 529, 606, 616; MR 1916

in Iceland....................................... B 330,491, 616

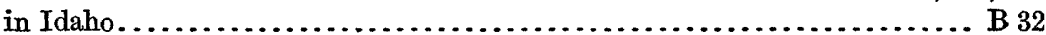

in Illinois..... W 164, 364; A $17 \mathrm{II} \mathrm{h}$; B 32, 330, 438, 491, 506, 616; GF 81, 188, 195,

$200 ; \mathrm{S} 3$

in Indiana........................ W 254; A 18 Iv b; B 32, 330, 491, 616

in Iowa................................. W 293, 364; B $32 ; \mathrm{GF} 145,200$

in Italy....................................... B $330,491,616$

in Java......................................... B 330, 491, 616

in Kansas............. W 145, 153, 258, 273, 345; P 32; B 32, 330, 491, 606, 616

in Kentucky............... W 102, 164, 233, 364; B 32, 330, 491, 606, 616, 661 d

in Louisiana............................. W 101; P 46; B 330, 491, 616

in.Maine............ W 102, 114, 144, 223, 258, 364; B 32, 330, 491, 616; GF 149

in Manitoba................................................. 25 
Analyses of ground water, in Maryland. ................ B 32, 138; GF 136 in Massachusetts............................. W 102, 110, 144; B 32 in Mexico. B 529 in Michigan....... W 31, 102, 145, 364; B 32, 330, 491, 606, 616, 625; GF 155, 205 in Minnesota. .............. W 102, 256; M 25; B 32, 330, 491, 616, 625; GF 201 in Mississippi............................... W 159, 364; P 46; B 32 in Missouri.............. W 102, 145, 195, 364; B 32, 330, 438, 491, 606, 616, 625 in Montana.......... W 221, 345, 364, 400 b; B 32, 330, 491, 529, 606, 616; GF 56 in Nebraska....................................... 184, 215 in Nevada....... W 364, 365, 423, $425 \mathrm{~d} ; \mathrm{P} 95$ a; B 32, 330, 491, 529, $530 \mathrm{r}, 540 \mathrm{n}$, $540 \mathrm{p}, 606,616$ in New Hampshire W 102,$144 ;$ B 32 in New Jersey. B 32, 138; GF 191 in New Mexico...... W 123, 158; 188, 275, 343, 364, 422, 425 a; B 32, 330, 491, 616, 618; GF 207 in New South Wales. B $330,491,616$ in New York... W 102, 108, 110, 144, 145, 364; P 44; B 32, 330, 491, 616; MR 1916; GF 190

in New Zealand B $330,491,616$ in North Carolina. . . . . . . . . . . . W 160, 258, 364; B 32, 138, 330, 491, 616; S 5 in North Dakota....................... 25; B 32; A 17 m g; GF 168 in Ohio .............. W 259; A $18 \mathrm{Iv} \mathrm{b,} 19$ Iv b; B 32, 330, 491, 616; GF 197 in Oklahoma............................... W 148, 345, 364; B 606 in Oregon......................................... $220 ;$ B 32 in Pennsylvania....................... W 108, 110, 364; B 32, 330, 491, 616 in Philippine Islands.............................. B 330,491, 616 in Rhode Island.................................. W 102, 144; B 32 in South Carolina........................ W 258, 364; P $90 \mathrm{~h} ; \mathrm{B} 32,138$ in South Dakota..... W 227; A 17 II g, $21 \mathrm{Iv} \mathrm{b;} \mathrm{P} \mathrm{32;} \mathrm{B} \mathrm{32;} \mathrm{GF} \mathrm{107,} \mathrm{108,} \mathrm{164,} 165$ in Switzerland.................................... B 330, 491, 616 in Tennessee..................... W 164, 364; B 32, 330, 491, 529, 616 in Texas. W $66,141,190,276,317,335,343,364,375 \mathrm{~g}$; A 18 II b, 21 vп; P 46; B 32; GF 166, 194; S 1

in Utah. W 157, 199, 217, 277, 333, 364; B 32, 330, 491, 616

in Vermont. W $102,114,144 ; \mathrm{B} 32$ in Virginia................... W 258,$364 ; \mathrm{B} 32,330,491,616$; GF 80 ; S 8

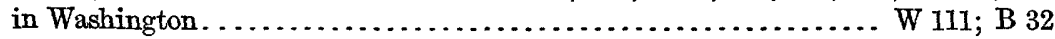
in West Indies............................................... $\mathrm{S} 10$ in West Virginia.............................. W 145, 364; B 32; GF 179 in Wisconsin ...................................... 32 ; GF 140 in Wyoming. $\mathrm{W} 364,425 \mathrm{~b} ; \mathrm{A} 9 \mathrm{~d}, 21 \mathrm{rv} \mathrm{b}$; P 32; B 32, $47,330,364,491,616 ;$ GF 107, 173 in Yellowstone National Park.................. A 9 d; B 47, 330, 491, 616 sanitary. $\mathrm{W} 31,144,195,256 ; \mathrm{S} 3$ methods of........................................... W 193

See also Analysis, Pollution, Purification, and Quality.

Analyses of incrustations on well screens....................... W 256, 293 Analyses of mine waters........................... W 364; B 330, 491, 529, 616 in Arizona................................................... 364 in California........................................... $364 ;$ B 529 in Colorado........................................... W $364 ;$ B 529 in Germany ................................................. B 529

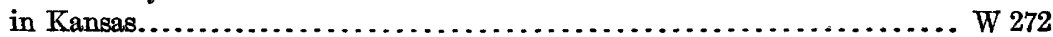


Analyses of mine waters, in Mexico . . . . . . . . . . . . . . . . . . . . . . 529

in Michigan.......................................... $364 ;$ B 625

in Minnesota. . . . . . . . . . . . . . . . . . 625

in Missouri............................................. $364 ;$ B 625

in Montana.................................. W $345 \mathrm{~g}, 364 ;$ B 529

in Nevada...................................... $364 ;$ B 529

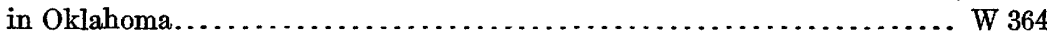

in Tennessee....................................... W 364; B 529

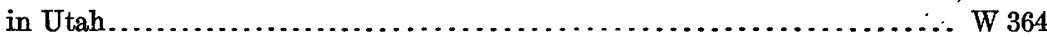

Analyses of mineral waters, bibliography of................ MR $1913 \mathrm{II}$ h, 1916

Analyses of soil, mechanical, methods of......... W 67, 104, 136; A 19 II b; P 44

Analyses of tufa formed by hot springs..................... W 145; A $9 \mathrm{~d}$

Analyses of water, accuracy of........................... 236, 274

assays compared with........................................ 398

computation of..................................... 151, 236,398

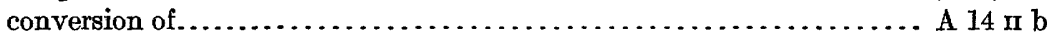

equivalents for............................................ 1911 п $\mathbf{n}$

industrial application of.................................. 274

interpretation of......................... W 274, 398; B 479, 606, 616

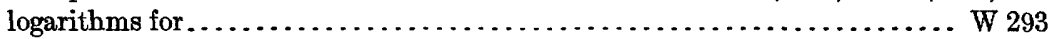

detecting errors in ........................................ 236

expression of ............... W 236, 274; A $14 \mathrm{In} \mathrm{b;} \mathrm{B} \mathrm{47,} \mathrm{330,} \mathrm{479,} \mathrm{491,} 616$

reaction coefficients for................................... W 274

Analysis of water for acidity .................................... 236

for albuminoid ammonia.................................. B 47

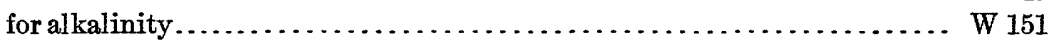

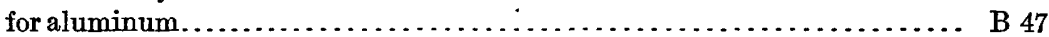

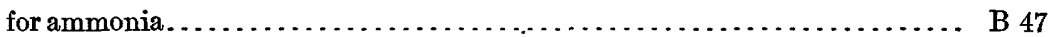

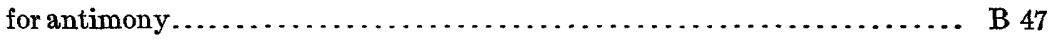

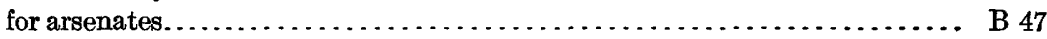

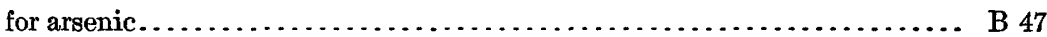

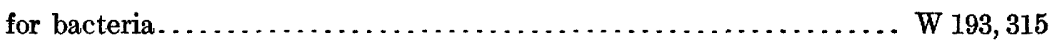

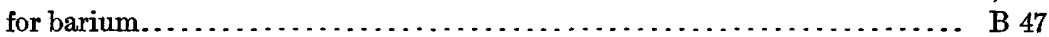

for bicarbonates......................................... 151, 236

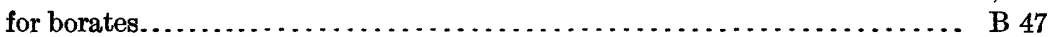

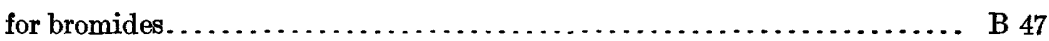

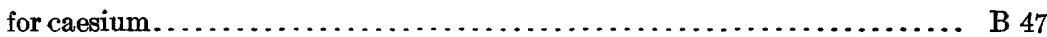

for calcium.......................................... 236 B 47

for carbonates.................................. 151, 236; B 47

for chlorine............................... 144, 151, 236; B 47

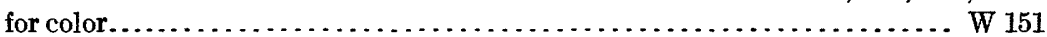

for copper.................. B 47

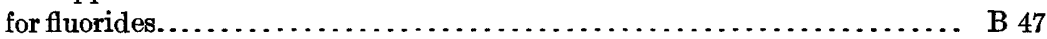

for hardness. . . . . . . . . . . . 151

for hydrogen sulphide.................... B 47

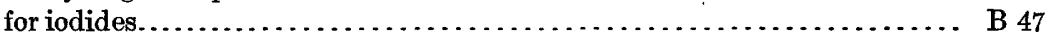

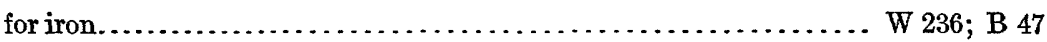

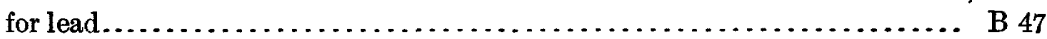

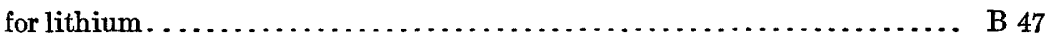

for magnesium...................................... 236 ; 47

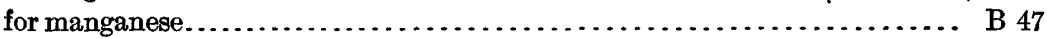

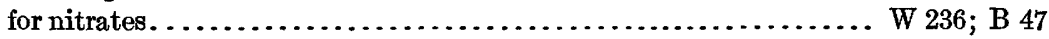


Analysis of water for phosphates............................ B 47 for potassium.................................... 236 ; B 47 for radioactivity ........................... B 395; MR 1913 In h

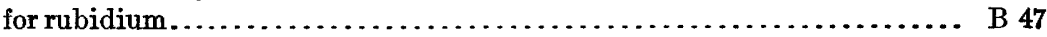
for silica..................................... 236; B 47 for sodium..................................... 236 ; B 47

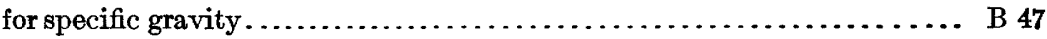

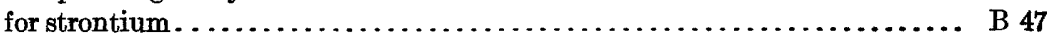
for sulphates...................................... 151,236 ; 47

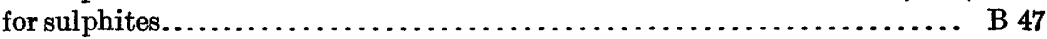
for sulphur............................... B 47

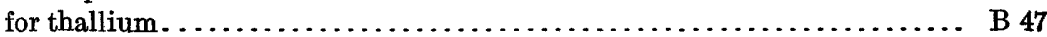

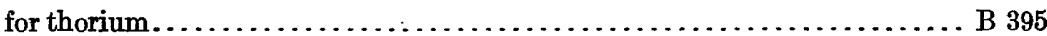

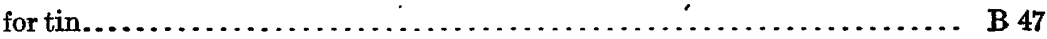

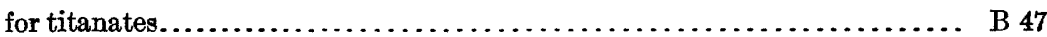

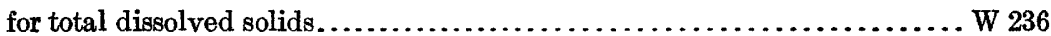

for total suspended solids.................................... 236

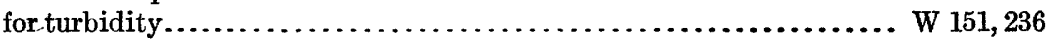

methods of............................ W 193, 236, 254, 320; B 47

in field.................................. 151, 193; B 47

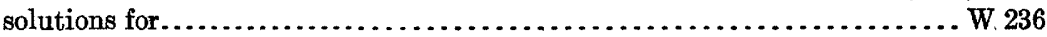

Anamorphism, zone of, ground water in relation to................... M 47

Annual recharge of ground water. See Quantity of ground water.

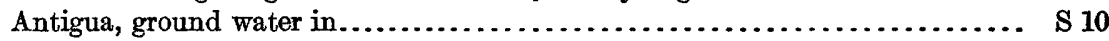

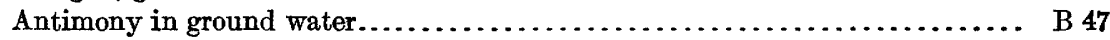

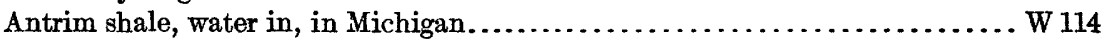

Apparatus for making assays of water.............................. 151

for making mechanical analysis of soil.......................... W 67

for recording water levels in wells .................... W 155; A 19 In b

for separating the emanation of uraninite in water................ B 395

for testing color of water..................................... 151

for testing gases in ground water............................. B 395

for testing solids in water............................ $137,138,139$

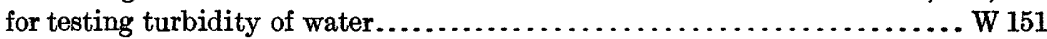

for testing windmills................................. $20,41,42$

Apparatus for measuring artesian pressure......................... W 145

deflection of wells............................................ W 257

discharge of wells and springs, weirs........................ 150, 200

evaporation of water from sand......................... W 294; A 19 in b

flow of ground water...................... W $67,110,112,140,141 ;$ P 44

flow of water and air through various media.................... A 19 In b

percolation of water.......................................... 155

radioactivity in water.......................... B 395; MR $1913 \mathrm{~m} \mathrm{~h}$

rate of rise of water in wells..................................... 140

water retained by soils................................... A 19 In b

Apparatus showing flow of water due to settling of sediments.......... A 19 II b

loss in head by friction in sands................................. W 258

loss in head of artesian water........... W 12,54; A 17 Ir g, 19 Iv c; P 17, 32, 52

Aquifers, use of term .......................................... 293

See also specific formations.

Arapahoe formation, water in, in Colorado.................... M 27; P 32

Archean formations. See under Pre-Cambrian; also see specific formations.

Archimedes limestone, water in, in Arkansas. ..................... 114, 145

Areas of ground-water discharge, methods of determining................. W 423 
Arey, M. F., Underground water resources of Iowa.................... W 293

Arikaree formation, water in, in Colorado...................... P 32 water in, in Nebraska .............. W 425 b; A 19 rv c; P 17, 32; GF 87,88 in South Dakota................................. W 227; P 32 in Wyoming.......................................... $425 \mathrm{~b} ; \mathrm{P} 32$ Aristotle on origin of ground water.......................... A 14 II b

Arizona, absorption of water in.................................. W 104

areas, Bisbee quadrangle............................... GF 112

Clifton quadrangle.................................. GF 129

Gila Valley ............................................ 104

Globe quadrangle................................... GF 111

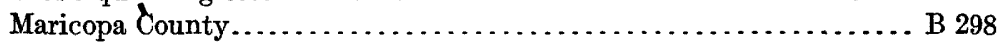

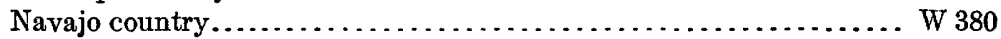

northeastern............................................ 380

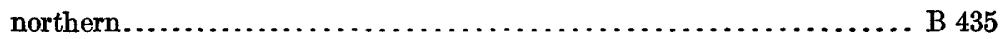

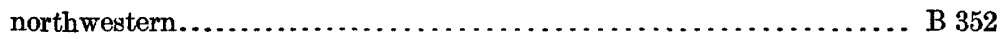

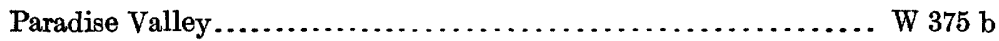

Patagonia Mountains.................................. B 582

Phoenix and vicinity........................... $2,136,375 \mathrm{~b}$

Pima Indian Reservation................................... W 104

Salt River Valley................................... 136

San Bernardino Valley................................ W 320

San Pedro Valley............................ $320 ;$ A 21 rv a

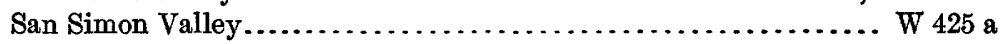

Santa Rita Mountains..................................... B 582

southeastern....................................... W 320,425 a

Sulphur Spring Valley .................................. 320

west-central................................................ B 352

artesian water in................ W 320,380,425 a; B 435; A 21 rv a; GF 112 bibliography of ground water in.......................... W 149, 163

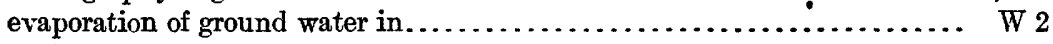
infiltration ditches in..................................... W 104 irrigation with ground water in.......... W 104, 136, 320, 375 b, 425 a; A 16 Ir e mine water in............................... W 364; B 582; GF 112 mineral water in........................ B 32; MR 1883-1916; GF 129 perched water in..................................... W 320 pumping in, cost of............................... 104, 136, 320 quality of ground water in .........W 104,320,364, $375 \mathrm{~b}, 425$ a; B 32; GF 129 salt water in....................................... W 136; GF 129

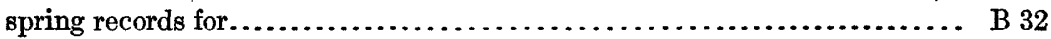
springs in, thermal......................................... B 32,582 temperature of ground water in ................. W 320; B 32,352, 582

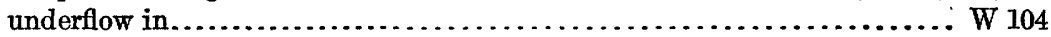
watering places in ................................ $380 ;$ B 352 well at Adamana, record for............................. B 540 p wells in, blowing.............................................. 258

cost of.............................................. W 320

records for................................... W 57, 149; B 298

Arkansas, areas, all of State..................................... 102

areas, Hempstead County................................ B 298

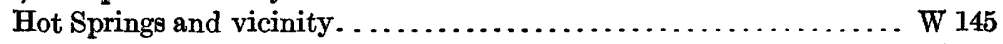

northeastern ........................................ W 399

northern..................................... 114,145

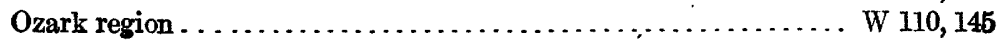


Arkansas, areas, southern

W 114; P 46

Tahlequah quadrangle.

GF 122

Winslow quadrangle.

W 145; GF 154

artesian water in.

W 114, 145, 399; P 46

bibliography of ground water in . ..................W 114, 120, 149, 163

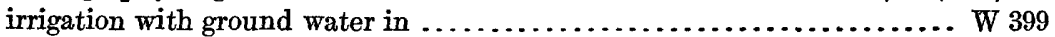

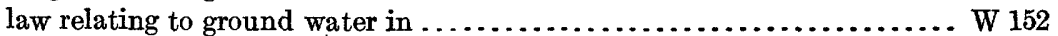

mineral water in................. W 114, 145; B 32; MR 1883-1916; GF 154

public water supplies in . . . . . . . . . . . . . . . . . . . . . . . 46

quality of ground water in . . . . . . . . . . . . . . . .

$145,364,399 ;$ B 32, 330, 491, 606, 616; P 46; GF 154

radioactive waters in................................ MR $1913 \mathrm{~m} \mathrm{~h}$

rice irrigation in, history of. . . . . . . . . . . . . . . . . . . . . . . . W 399

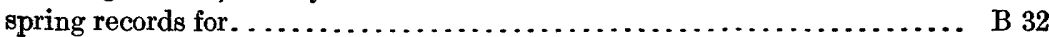

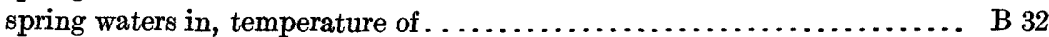

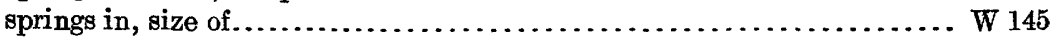

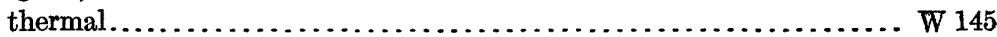

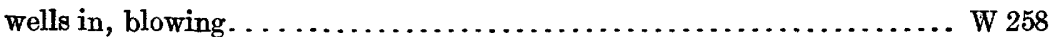

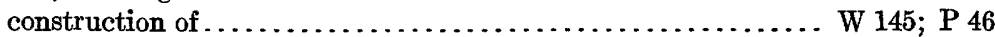

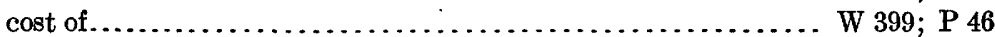

drainage into............................................. 160

records of ............................ 5 57, 149, 399; B 264, 298

Arnold, Ralph, Santa Cruz folio, Cal............................. GF 163

Arsenic in ground water ...................... W 338; B 47; MR 1911 In $n$

Artesian basins produced by settling of strata.................A 21 IV c

Artesian pressure, factors influencing ..................... A 5c; B 319

methods of measuring ................................... 118,145

Artesian springs............................ W 90,255, 278, 338; A 21 IV c

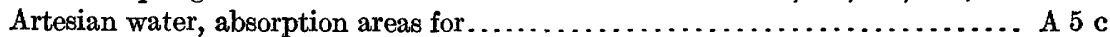

apparatus showing loss of head in....W 12, 54, 227; A $17 \mathrm{II} \mathrm{g,} 19 \mathrm{rv} \mathrm{c} ; \mathrm{P} 17,32,52$

barometric pressure in relation to . . ................... B 319; GF 156

bibliography of .......................................... W 54

bounties for discovering .................................. 122

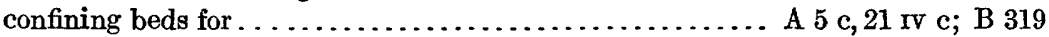

conservation of .......................................... 142 ,

$145,158,219,225,234,256,278,293,400 \mathrm{~b}, 423,425$ a; S 8,12

cost of recovering, in California ......................... W 222, 398

in Mentana......................................... $400 \mathrm{~b}$

in Nevada................................... W $375 \mathrm{~d}, 423$

criteria useful in prospecting for.......................... A 5 c; S 8

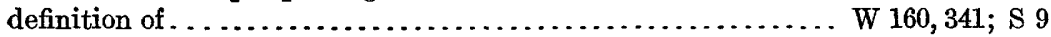

deformation in relation to................................... B 606

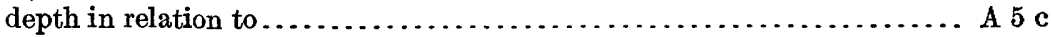

dip of strata in relation to................................. $5 \mathrm{c}$

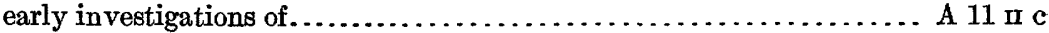

escape of, in wells............................... 118,257; A 5 c

fallacies regarding............................. W 278,$380 ;$ B 319

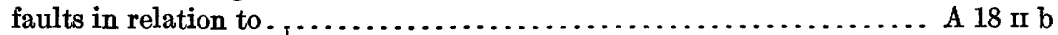

gas in, flows produced by .................................. $5 \mathrm{c}$

head of, atmospheric effects on........................... 319 ;F 156

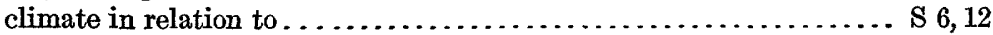

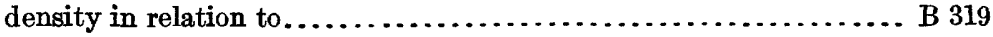

depth in relation to............................ A 5 c; GF 168 
Artesian water, head of, fluctuations in .... W 34, 101, 240, 256, 319; GF 156; S 6, 8, 12

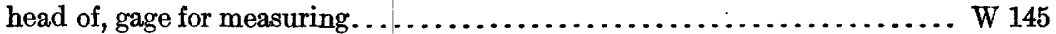
loss in W $59,60,90,137,138,139,142,145,219,240,255,256$, 293,425 a; A 17 II g; GF 96, 97, 99, 100, 113,114, 156; S 8, 12 measurement of..................................... 118, 145

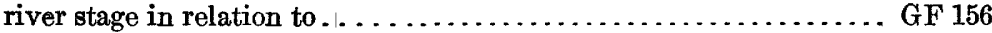
Salton Sink in relation to............................... W 225 temperature in relation to ............................... B 319 water table in relation to $\ldots \ldots \ldots \ldots \ldots \ldots \ldots \ldots$ W 256, 293; A 5 c; S 9

"high-level flows" of .................................... W 320

hot, in Idaho . . . . . . . . . . . . . . . . . . . . . . 45

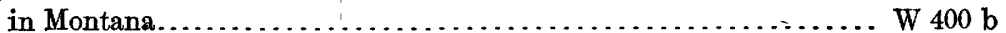

hot springs in relation to.............................. $400 \mathrm{~b}$

in Alabama. . . . . . . . . . . . . . . . . 2

in Algeria.................................... 11 Ir $\mathrm{c}$

in Algonkian formations. . . . . . . . . . . . . . . . 256

in Antigua. . . . . . . . . . . . . . . . . . . . 10

in Arapahoe formation ....................................... 27 in Arkansas................................... 114, 145, 399; P 46 in Arizona...................... W 320, 380, 425 a; A 21 rv a; GF 112 in Atlantic Coastal Plain............................ W 258; B 138 in basins fed by joints ................................... B 319 in bedding planes....................................... B 319 in Benton formation....................... GF 96, 99, 100, 113, 114165 in Boone formation........................................... 148 in California......W 45, 59, 60, 89, 137, 138, 142, 146, 181, 213, 222, 224, 255, 278, 398 ; GF 138, 163, 193; S 6 in Calvert formation...............................F 136, 137, 152 in clay ......................................... 21 vII in Colorado........ W 9, 240; A 16 II f, 17 II f; M 27; P 32, 52; B 265, 350, 531 c; GF $36,58,68,71,135,198,203$ in Connecticut.................................. W 232, 374, 397 in contact planes......................................... B 319 in crystalline rocks..... W 106, 145, 160, 232, 374, 397; A 5 c; GF 149, 157, 158; S 9 .in Dakota sandstone.... W 6, 12,34, 215, 216, 227, 256; A 16 II f, 17 II f, 17 II g, 18 Iv c, 19 rv c, 21 rv c; M 25,$27 ; \mathrm{P} 17,32,52,65 ; \mathrm{B} 350 ; \mathrm{GF} 68,71,85$, $87,88,96,97,100,107,108,113,114,127,128,135,150,156,164,165,168$ in Deadwood sandstone. . ...................... W 227; P 32; GF 164 in débris-filled basins... W 142, 181, 219, 225, 240, 277, 278, 320, 333, 343, $375 \mathrm{~d}, 423$ in Denver formation. ....................................... M 27 in deserts. . . . . . . . . . . . . . . . . . . . . . . . 277 in District of Columbia........................... B 138; GF 70, 152 in Delaware...................................... B 138; GF 137 in eastern United States................................ W 114 in Ellensburg formation........................... W 55, 118; GF 86 in faults................................................ B 319 in fissured rocks.......................... W 160, 232, 374, 397; A 5 c in Florida. . . . . . . . . . . . . . . . . . . . . . . . . . . . . . . . . . W 319

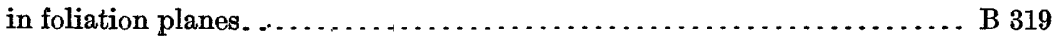
in Fox Hills formation. . . . . . . . . . . . . . . . . . . . . . . 27 in Fredericksburg group .............................. A 21 vII in frost crusts. . . . . . . . . . . . . . . . . . . . . . . . . . . . . . . . . B 319 in Galena limestone......................... W 256, 293; GF 145; 99 in Georgia. ............................... W 67, 341; B 138; 54,11 
Artesian water, in glacial drift.... W 182, 183, 254, 256, 293; A 17 II g, 17 II h, 18 Iv b, 19 Iv b; M 38; P 44; GF 96, 97, 99, 100, 113, 114, 117, 140, 155, 157, 165, 168; S 9 in Glen Rose formation................................ A 21 vII in gneiss...................................... 106; GF 157 in Grand Falls chert...................................... 148 in Hawaii.............................. 318,336, 373, 430; $\mathrm{S} 12$ in High Plains..................................... 21 Iv c in Idaho. ........................... 54, 78; B 199; GF 45, 103, 104 in Illinois.................. A 17 II h; M 38; B 438, 506; GF 67, 81, 195; S 3 in Indiana. . . . . . . . . . . . . . . . . . . . . 26, 114, 254; A 18 Iv b; GF 67

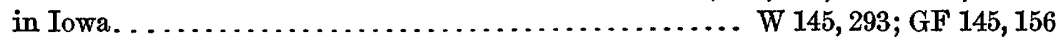
in joints............................. W 160, 232, 256; B 319 in joints confined by alluvium........................... B 319 in joints confined by sea water. . . . . . . . . . . . . . . . . . . . . B 319 in Kansas...................... W 6, 273; A 16 II f, 21 IV c; P 32; GF 148 in Kentucky. . ................................... 164, 233 in lacustrine deposits. ..................... W 217, 220, 277, 333, 425 a in Lakota sandstone. . . . . . . . . . . . . . P65; GF 107, 108, 127, 128, 150, 164 in Laramie formation. . . . . . . . . . . . . . . . . . . . . . M 27; GF 87 in lava.................................... 4, 55; B 252 ; 12 in lava and interbedded alluvium.................... W 4,54; B 199 in Lloyd sand ......................................... P 44 in Louisiana.................................... 101; P 46

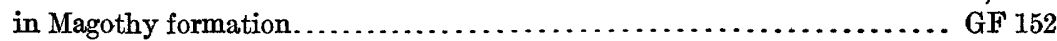
in Maine............................. 145, 223, 258; GF 149, 158 in Maryland.............. B 138; GF 23, 70, 136, 137, 152, 160, 179, 182, 204 in Michigan...................... W 30, 145, 160, 182, 183; GF 155, 205 in mineral crusts...................................... B 319 in Minnelusa formation............................. $128,150,164$ in Minnesota......................... W 114, 256; M 25; GF 117, 201

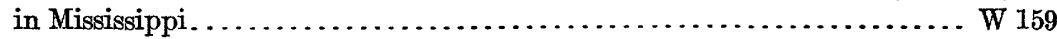
in Missouri............................... 195; B 438; GF 148 in Monmouth formation. . . . . . . . . . . . . . . . . . . . . . . 137 in Montana.................... W 221, $345 \mathrm{~g}, 400 \mathrm{~b}$; B 646; GF 55, 128 in Montana clays.......................................... 27 in Myrick formation................................. 375 g in Nebraska.............. W 12, 29, 184, 215, 216, 425 b; A 16 II f, 19 Iv c; P 17, 32; GF $85,87,88,108,156$

in Nevada W $289,365,375 \mathrm{~d}, 423,425 \mathrm{~d}$ in New Jersey............................. W 106; B 138; GF 157, 162 in New Mexico......................... W 123, 188, 343, 380, 422; GF 199 in New York. .....................W i10, 145; B 138; P 44; GF 169 in Newark sandstone.............................. W 232; GF 157 in Niagara limestone......................... A 19 Iv b; GF 145; S 9 in North Carolina.................................. 138; GF 80; S 5 in North Dakota. ................... A 17 II g; M 25; B 575; GF 117, 168 in Ohio........................ W 114, 259; A 18 Iv b, 19 Iv b; GF 197

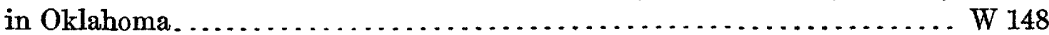
in Oregon........................... 78; 220, 231; B 252; GF 103 in Paluxy sand. . . . . . . . . . . . . . 21 vII in Pecos Valley, New Mexico.............................. W 158 in Pennsylvania............ W 106; B 138, $531 \mathrm{~d}$; GF 102, 121, 133, 160, 179, 189 in Pierre shale. ......................................... GF 165 in Platteville limestone............................... GF 145; S 9 
Artesian water in Pleistocene coastal clays

A 21 vir in Potomac group B 138; GF 137, 152 in Potsdam sandstone.......... W 256, 293; A 17 Ir h; M 38; GF 81, 140, 145; S 9 in preglacial channels. A 19 IV b in quartzite W 256 in Quaternary stream deposits.............................. W 6 in rocks overlain by drift. .............................. W 145, 256 in St. Peter sandstone.............. W 233, 256, 293; A 17 m h; M 38; B 438; GF $81,140,145,148 ; \mathrm{S} 9$ in sand at surface. W $145 ;$ B $319 ;$ P 44 in Sioux quartzite overlain by glacial drift................... W 256

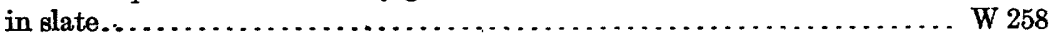
in solution passages. . . . . . . . . . . . . . . . . . . . . . . . . . . B 319 in South Carolina. ................................. B $138 ;$ P $90 \mathrm{~h}$ in South Dakota. ....... W 34, 90, 227, 428; A $17 \mathrm{II} \mathrm{g,} 18 \mathrm{IV}$ c; $21 \mathrm{Iv} \mathrm{b,} \mathrm{P} \mathrm{32,}$ 65 ; B 575; GF 85, 96, 97, 99, 100, 107, 108, 113, 114, 128, 156, 164, 165 in trap rocks. B 319 in Tennessee. W 164 in Terrell reservoir.................................... 21 viI in Texas. $\mathrm{W} 13,71,190,191,276$, $335,375 \mathrm{~g}$; A 18 II b, $21 \mathrm{vIr}$; B 164; GF 42, 64, 76, 194; S1 in Triassic formations......................... W 232; M 27; GF 157 in Trinity sand . . . . . . . . . . . . 76 in unconfined horizontal beds..................... W 145; B 319; P 44

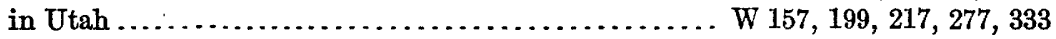
in valley fill. ................................. $89,137,138$, $142,181,199,219,220,222,225,240,277,278,320,333,375$ d; GF 163

in Vermont W 114 in Virginia.................................. 138; GF 23, 70, 80; 8 in Wasatch formation.................................. P 53 in Washington................. W 4, 55, 75, 118, 316; B 108; GF 86, 106 in weathered rocks beneath clays. ......................... B 319 in West Virginia..................................... 160,179 in Wisconsin..................................... GF 140, 145; S 9 in Woodbine sand . ..................................... 21 vII in Wyoming....................... W $425 \mathrm{~b} ; \mathrm{A} 21 \mathrm{rv}$ b; P $32,51,53$, 65 ; B 285, 364, 543, 641, 656; GF 107, 127, 128, 141, 142, 150, 173 irrigation with, developments of, about $1890 \ldots \ldots \ldots \ldots \ldots \ldots$ A 11 m c; 13 mI a

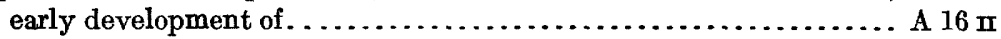

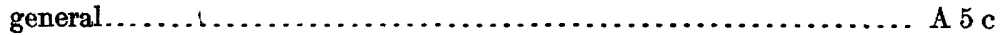
in Algeria....................................... $136 ; 11$ II c in Arizona .......................... W 425 a; A 21 rv a; B 435 in California.............. W 137, 138, 139, 142, 219, 222, 225, 278, 398 in Colorado....................................... W 240

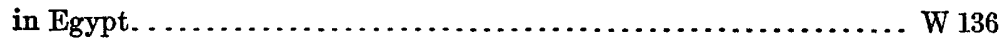
in Georgia. . . . . . . . . . .

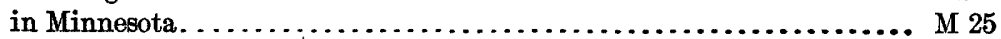

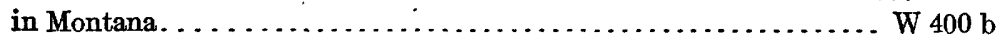
in Nevada. ................................. W $375 \mathrm{~d}, 423$ in New Mexico............................... W 158, 425 a in North Dakota............................... A $17 \mathrm{n} \mathrm{g} ; \mathrm{M} 25$ in South Dakota........................ A 16 II e, $17 \mathrm{mg}$, $18 \mathrm{IV} \mathrm{c}$ in Texas. ................................ $13,71,375 \mathrm{~g}$ in Washington $, \ldots, \ldots \ldots, \ldots, \ldots, \ldots, \ldots, \ldots, \ldots, \ldots, \ldots, \ldots, \ldots$, W 55 
Artesian water, lakes produced by ........................ A 18 rv c law relating to............................... 55,$78122 ; \mathrm{S} \mathrm{3,12}$ leakage of . . . . . . . . . . . . . 118,257 ; 5 c methods for detecting .......................... $118 ;$ A 5 c maps showing. See under Maps.

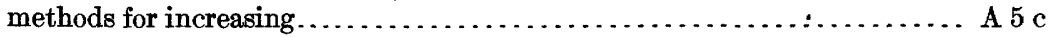

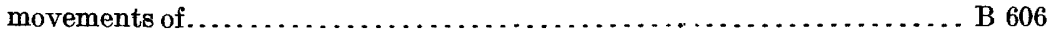

See also under Movements of ground water.

origin of..................W 101, 158; A 5 c; M 27; B 319; GF 97, 117 "pit flows" of . . . . . . . . . . . . . . . . . . . . . . . . . . . . . . . 277

perched bodies of ........................................ W 320 power developed from. ................... W 67; A 17 II g; GF 168; S 4 principles of ................ W 114, 118, 195, 254, 257, 341, 365, 399; B 319; A 5 c, 14 Ir a, 21 Iv c, 21 vIr; M 27 ; P 44, 46; GF 68, 71, 86; S 2,9 significance of term....................................... W 160 temperature of. See Temperature of artesian water.

unusual conditions of................................ W 145, B 319

"Artesian wedges," nature of. . . . . . . . . . . . . . . . . . . . . . . . . . B 199

Artesian wells, clogging of..................................... W 293

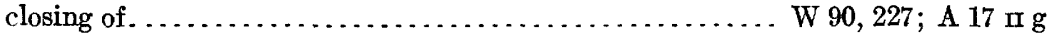
diameters of, in relation to yield . . . . . . . . . . . . . . . . . . W 293 detecting of flows in .......................................... 118 gases in relation to yield of ................................ $5 \mathrm{c}$

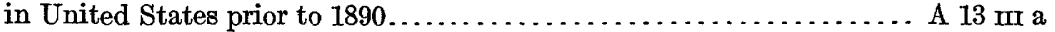
interference of........................................ 19 II c large yields of ............................. 67,158 ; GF 205; S 12 laws of flow in ....................................... 19 II $\mathrm{c}$ leaks in ....................................... 118, 257; A 5 c

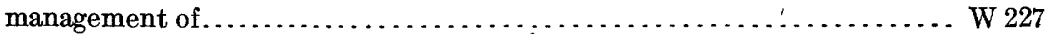
methods of controlling. . . . . . . . . . . . . . . . . $5 \ldots \ldots \ldots \ldots$. temperature of water in relation to yield of . . . . . . . . . . . . . . . A 5 c yield of, diameters in relation to............................ W 293

effect of density on . . . . . . . . . . . . . . . . . . . . . . . . . . . B 319

effect of gases on . . . . . . . . . . . . . . . 5 c effect of temperature on ........................... A 5 c; B 319 effect of weather on . . . . . . . . . . . . . . . . . . . . . . . . 258 methods of measuring...................... W 110, 219, 240, 257 principles of . .......................................... 293 seasonal variations in. . . . . . . . . . . . . . . . . . . . 12 See also Artesian water, and Well construction.

Ashley, G. H., Ditney folio, Ind................................. 84 Geologic structure of Punxsutawney, Curwensville, Houtzdale, Barnesboro, and Patten quadrangles, Pa. . . . . . . . . . . . . . . . . B $531 \mathrm{~d}$ Nicholas quadrangle, W. Va............................... W 145 Water resources of the Middlesboro-Harlan region of southeastern Kentucky. W 110 Asia, irrigation with ground water in . ........................... W 1

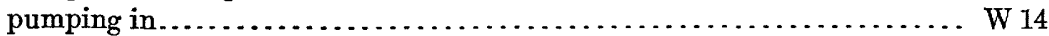

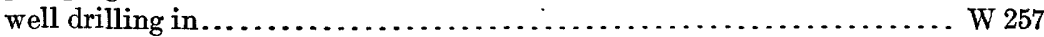
See also China, India, Japan, Persia, Russia, and Turkestan.

Aspirator for mechanical analysis of soil. . . . . . . . . . . . . . . . . . . W 67

Assays of ground water in Arkansas. . . . . . . . . . . . . . . . . . . . . . . W 399

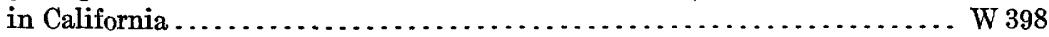

in Georgia $\ldots \ldots \ldots, \ldots, \ldots, \ldots, \ldots, \ldots, \ldots, \ldots, \ldots, \ldots, \ldots, \quad s 4$ 
Assays of ground water in Indiana.......................... W 254

in Kansas ................................................ W 273

in Kentucky . . . . . . . . . . . . . . . . . . . . . . 233

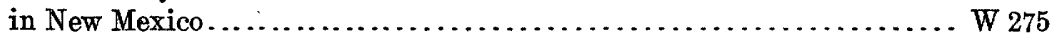

in North Carolina . . . . . . . . . . . . . . . 5

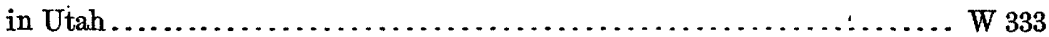

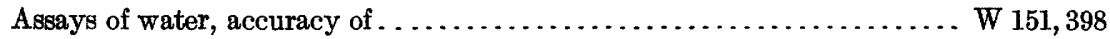

analyses compared with .................................... 398

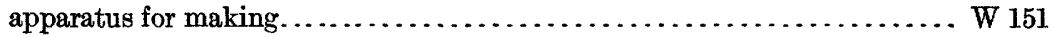

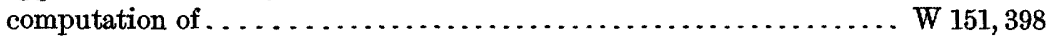

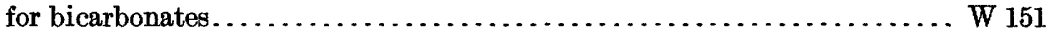

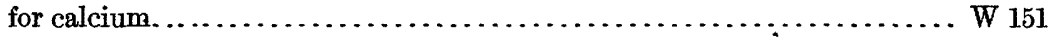

for carbonates. . . . . . . . . . . . . . . . . . . . . . . 151

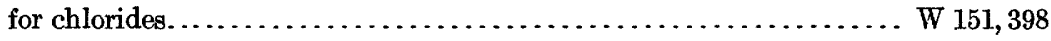

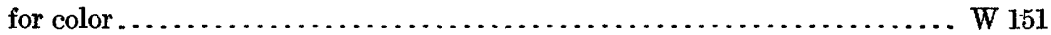

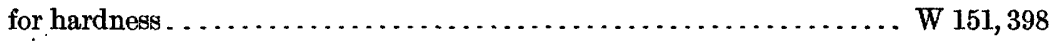

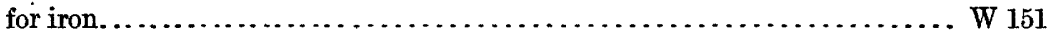

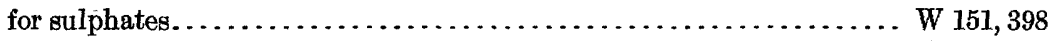

for turbidity...................................... 151,398

methods of making................................. 151, 193, 398

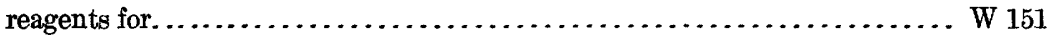

Atlantic Coastal Plain. See individual States.

Atmospheric effects on wells. See under Wells.

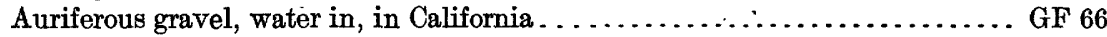

Austin formation, water in, in Texas................ W 276; A $13 \mathrm{~m} \mathrm{b;} \mathrm{GF} 64$

Australia, bibliography of ground water in ........................... 163

deep wells in . ............................................ 257

Austria-Hungary, bibliography of ground water in.................. W 163 quality of ground water in.................... B 330,491,616; MR 1916 radioactive waters in ..................................... $1913 \mathrm{~m} \mathrm{~h}$

B.

- Bacteria in ground water ............... W 193, 256, 259, 335, 341, 398, 399; S 11

Bacteriological analysis of water............................. W 193, 315

Bailey, E. H. S., Stream pollution by mine waters in southeast Kansas ..... W 273

Ball, S. H., Southwestern Nevada and eastern California................ B 308

Baluchistan, ground water in, bibliography of................... W 163

Barbour, E. H., Wells and windmills in Nebraska................... W 29

Barium in ground water................ W 254,338, 399; B 47; MR 1911 In n

Barker, F. C., Irrigation in Mesilla Valley, N. Mex ................... W 10

Barnett, V. H., Standing Rock and Cheyenne River Indian reservations, N.

Dak.-S. Dak ............................................ B 575

Barometric effects on wells. See under Wells.

Barriers to ground water. See under Dams.

Basalt. See Lava.

Bascom, Florence, Water resources of Philadelphia district, Pa.-N. J.-Del.... W 106

Philadelphia folio, Pa.-N. J.-Del ........................... GF 161

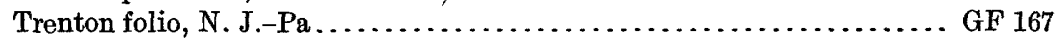

Basins. See Débris-filled basins.

Baskets, swinging, for lifting water. . . . . . . . . . W 1

Bastin, E. S., Eastport folio, Maine......................... GF 192

Economic geology of Gilpin County and adjacent parts, Colo............ P 94

Penobscot Bay folio, Maine................................. GF 149

Rockland folio, Maine.................................. GF 158

$$
56122^{\circ}-18-\text { W\$P } 427-5
$$


Batesville sandstone, water in, in Arkansas.................... W 114, 145

Bayley, W. S., Passaic folio, N. J.-N. Y........................... GF 157

Raritan folio, N. J . . . . . . . . . . . . . . . . . . . . . . . . . . . GF 191

Underground waters in Maine............................. 114

Underground waters of southern Maine...................... W 223

Wells and springs in Maine.............................. W 102

Beal, C. H., Geologic structure in Cushing oil and gas field, Okla.', and its rela-

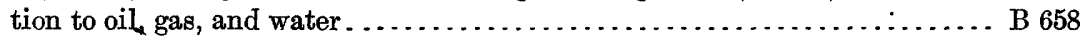

Beaumont clay, water in, in Texas............................... W 335

Beckwith formation, water in, in Wyoming .................... P 56

Bedding planes, water in ............................ 114, 254; B 319

Beekly, A. L., Geology of Standing Rock and Cheyenne River Indian reser-

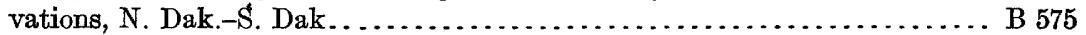

Belgium, ground water in, bibliography of ................... W 163 radioactive water in.......................................... 1913 II $\mathrm{h}$

Benton formation, water in, in Kansas .................... W 273; P 32 in Nebraska.................................. W 216; P 32 in South Dakota............. W 90, 227; P 32; GF 96, 97, 99, 100, 113, 114, 165 in Wyoming . . .............................. P 32; B 364; CF 173

Berea grit, water in, in Michigan.............................. W 31 water in, in Ohio.......................................... W 114

Berea shale and grit, water in, in Michigan.................... W 30, 114

Bermuda Islands, bibliography of ground water in.................. W 163

Bibbins, Arthur, Patuxentffolio, Md.-D. C.......................... GF 152

Tolchester folio, Md....................................... 204

Bibliography, absorption of water ............................ 294 analyses of American mineral water................. MR $1913 \mathrm{Ir} \mathrm{h}, 1916$ analyses of water.................. B 330, 491, 606, 616; MR $1913 \mathrm{II} \mathrm{h}, 1916$

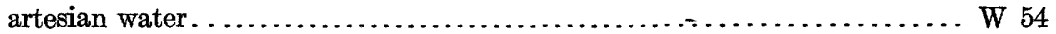

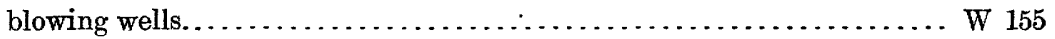
deep wells. . . . . . . . . . . . . . . . . . . . . 57,61

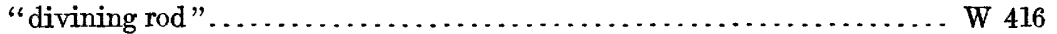

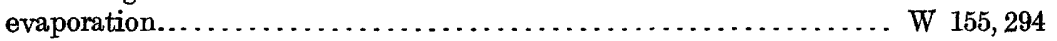

fluctuations of water table................................. 155

ground water, in Alabama ...................... W 114, 120, 149, 163

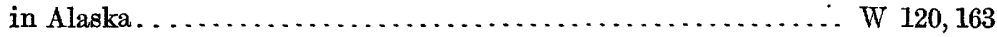

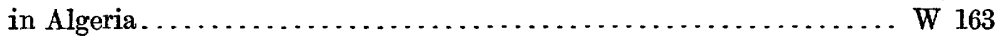

in Arizona...................................... 149,163

in Arkansas................................ 114, 120, 149, 163

in Australia . . . . . . . . . . . . . . . . . 163

in Austria-Hungary . . . . . . . . . . . . 163

in Baluchistan..................................... W 163

in Belgium. ........................................ 163

in Bermuda Islands....................................... 163

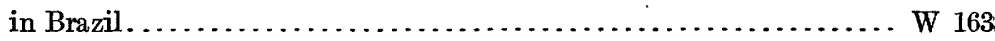

in British Columbia.................................. W 163

in California............................ $57,120,142,149,163$

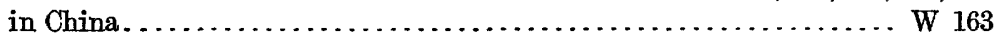

in Colorado.............................. W 57, 120, 149, 163

in Colorado River basin............................. W 340

in Connecticut. ............................. $114,120,163$

in Cuba............................................ W 163

in Delaware.............................. W 114, 120, 149, 163

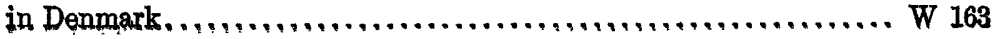


Bibliography, ground water, in District of Columbia.

$\mathrm{W} 114,120,163$

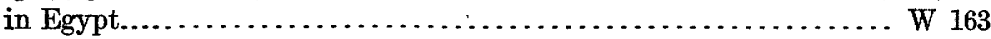

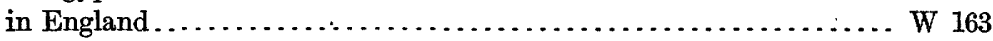

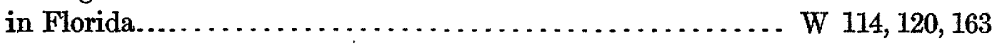

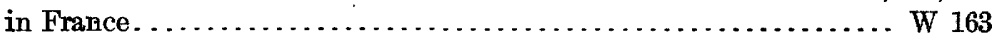

in Georgia........................... $57,114,120,149,163$; S 4

in Germany........................................... 163

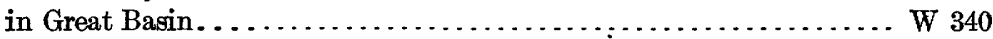

in Gulf of Mexico drainage basins. ....................... W 340

in Hawaii. . . . . . . . . . . . . . . . . . . . . . . . . . . . . . . 163

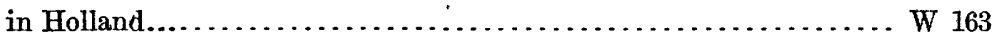

in Hudson Bay drainage basin............................ W 340

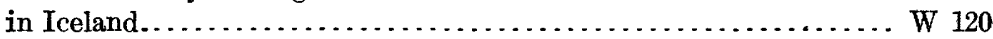

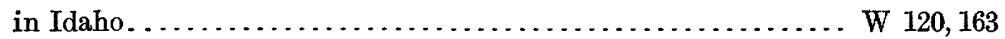

in Mlinois......................... $57,114,120,149,163,164$

in India......................................... 120,163

in Indiana................................... $57,114,120,149,163$

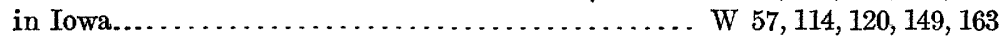

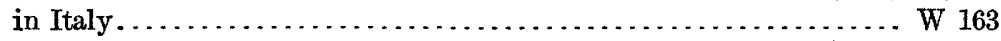

in Japan . . . . . . . . . . . . . . . . . 163

in Kansas................................ $57,120,149,163$

in Kentucky .................... $57,114,120,149,163,164,233$

in Louisiana. . . . . . . . . . . . . . . . . . . . . . . . 114, 120, 149, 163

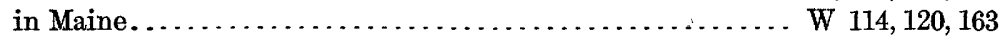

in Maryland............................. W 114, 120, 149, 163

in Massachusetts............................ W 114, 120, 163

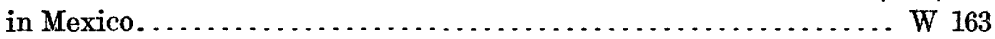

in Michigan............................. $57,114,120,149,163$

in Minnesota.......................... $57,114,120,149,163$

in Mississippi.............................. 114, 120, 163

in Mississip pi River basin............................. W 340

in Missouri............................... 57, 120, 149, 163, 340

in Montana....................................... 120,163

in Morocco............................................... 163

in Nebraska............................. W 61, 120, 149, 163

in Nevada........................................ 120,163

in New Hampshire............................ W 114, 120,163

in New Jersey ........................ W 61, 114, 120, 149, 163

in New Mexico............................ $61,120,149,163$

in New South Wales. . . . . . . . . . . . . . . . . . . . . . . . . . W 163

in New York........................... W 61, 114, 120, 149, 163

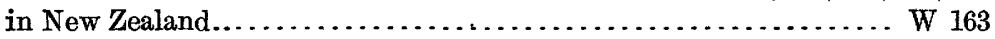

in Nicaragua......................................... 163

in North Atlantic drainage basins. ....................... W 340

in North Carolina...................... W 61, 114, 120, 149, 163; S 5

in North Dakota............................ W 61, 120, 149, 163

in Ohio.............................. $61,114,120,149,163$

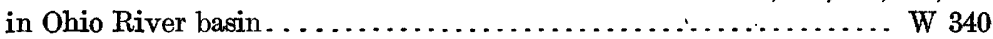

in Oklahoma.................................. W 120, 163

in Oregon........................................... 120,163

in Pacific slope basins. . . . . . . . . . . . . . . . . . . . . . . . . W 340

in Pennsylvania......................... $61,114,120,149,163$

in Porto Rico $, \ldots \ldots \ldots, \ldots, \ldots, \ldots, \ldots, \ldots \ldots, \ldots, \ldots, \ldots, \mathrm{W} 120$ 


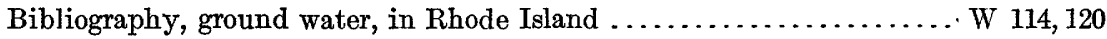
in St. Lawrence River basin.......................... W 340 in South Atlantic coast basins.......................... W 340 in South Carolina.......................... 61, 114, 120, 149, 163 in South Dakota............................. 6 61, 120, 149, 163

1 in Tennessee............................. $114,120,149,163,164$ in Texas................................ $61,120,149,163$

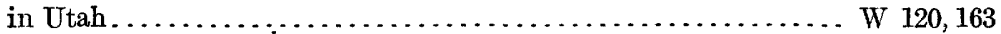

in Vermont...................................... 114, 120, 163

in Virginia............................. W 61, 114, 120, 149, 163

in Washington..................................... 120,163

in West Virginia.......................... W 61, 114, 120, 149, 163

in Wisconsin.............................. W 61, 114, 120, 149, 163

in Wyoming................................ $61,120,149,163$

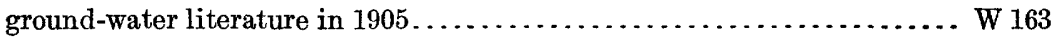

mineral waters, general.................... W 120, 163; MR 1905, 1916

mineral waters in United States............... W 120, 163; MR 1905, 1916 movements of ground water .............................. 19 In $c$

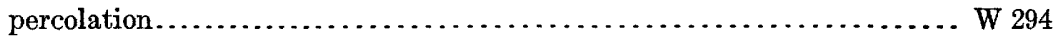

pumping appliances......................................... 343

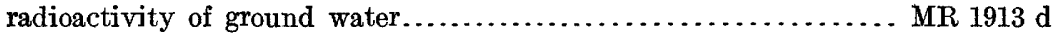

United States Geological Survey publications on ground water, 1879-1904. W 120

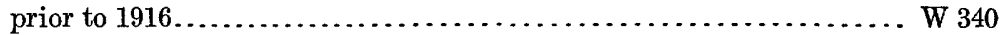

water in crystalline rocks................................. W 232

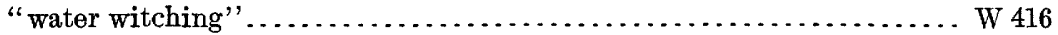

weirs............................................ 150, 200

Bicarbonates in ground water...........W 254, 259, 273, 338, 341, 398; MR 1911

Bighorn limestone, water in, in South Dakota...................... P 32

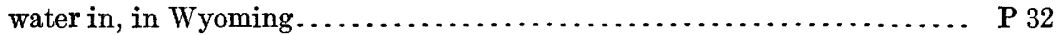

Bingen formation, water in, in Arkansas..................... $114 ;$ P 46

water in, in Louisiana...................................... W 114

"Birds-eye" limestone, water in, in Ohio......................... W 259

Black alkali in ground water.............................. W 320,343

Black Creek formation, water in, in North Carolina.................. S 5

Blackwelder, Eliot, Laramie-Sherman folio, Wyo.................. GF 173

Bleaching, quality of water for ......................... 254, 398

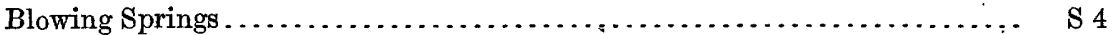

Blowing wells. See under Wells.

Boiler compounds.......................................... W 398

Boiler use of ground water. See under Quality and Analyses.

Bolson deposits. See Valley fill.

Bolsons. See Débris-filled basins.

Bone Valley gravel, water in, in Florida.......................... W 319

Boone formation, water in, in Arkansas.................. W 145; GF 122, 154

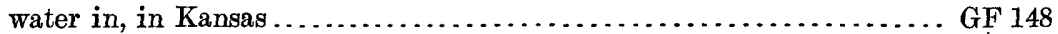

in Missouri......................................... 148

in Oklahoma $\ldots \ldots \ldots \ldots \ldots \ldots \ldots \ldots \ldots \ldots \ldots \ldots \ldots \ldots$ GF $122,132,154$

Borates in ground water................. W 338; B 47, 330, 491, 616; MR 1911 II n

Boring of wells. See under Well construction.

Boston formation, water in, in Arkansas............................. 145

"Bottom water," relation of, to oil ............................. B 658

Bounties for discovering artesian water.......................... 122 
Boutwell, J. M., Records of wells and springs in New Hampshire.......... W 102

Bowman, Isaiah, Well records on Long Island, N. Y.................... P 44

East St. Louis district, Illinois.............................. S 3

Problems of water contamination........................ 160

Flowing wells and municipal water supplies in southern Peninsula of Mich-

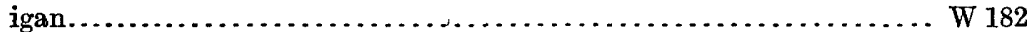

Well-drilling methods..................................... W 257

Bownocker, J. A., Columbus folio, Ohio.......................... GF 197

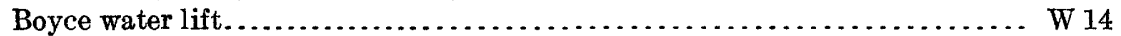

Boyd, David, Irrigation near Greeley, Colo ................... W 9

Branner, J. C., Santa Cruz folio, Cal................................ GF 163

Brazil, ground water in, bibliography of .............................. W 163

Breathing wells. See Wells.

Breccia, water in, in California................................... W 375 a

Brewing. See under Quality.

Brine. See under Quality and Salt water.

British Columbia, ground water in, bibliography of................... W 163

Bromides in ground water................ W 31, 233, 338, 398; B 47; MR 1911 in $\mathbf{n}$

Brown, C. W., Penobscot Bay folio, Maine....................... GF 149

Brownstown formation, water in, in Arkansas ..................... P 46

Brule clay, water in, in Colorado........................... P 32

water in, in Nebraska....................... W $425 \mathrm{~b} ;$ A 19 Iv c; P 17, 32

in South Dakota.................................. P 32

in Wyoming.......................................... $425 \mathrm{~b} ; \mathrm{P} 32$

Bryan, Kirk, Sacramento Valley, Cal........................ W 375 a

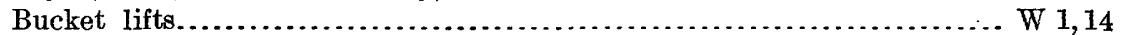

Burgoon sandstone, water in, in Pennsylvania.................. GF 133

Buried channels, water in ............................... W 273, 293

Burlington limestone, water in, in Illinois.................... 438,506

water in, in Iowa.......................................... 293

in Missouri........................................... W 195; B 438

Butts, Charles, Barnesboro-Patton folio, $\mathrm{Pa} \ldots \ldots \ldots \ldots \ldots \ldots \ldots \ldots$. . . . . . . . . 189

Birmingham folio, Ala........................................ GF 175

Ebensburg folio, Pa...................................... 133

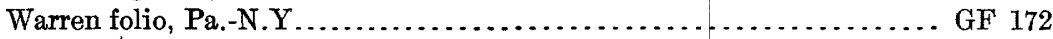

C.

Caesium in ground water......................................... 44

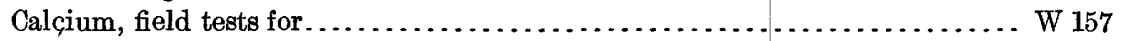

in ground water. See under Analyses, Analysis, and Quality.

Calculus applied to movements of ground water................... A 19 II $\mathrm{c}$

Califórnia, analyses of mine waters in . . . . . . . . . . . . . . . . . . . . . W 364

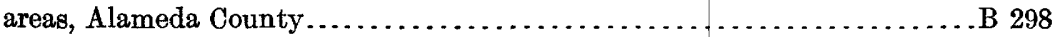

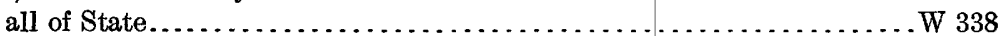

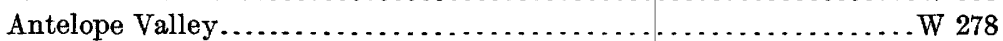

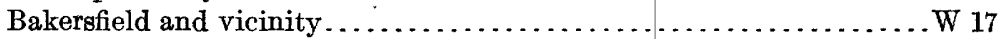

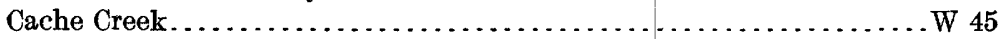

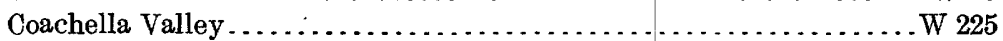

Coastal Plain of southern California. ............... W 137, 138, 139

Colfax quadrangle. . . . . . . . . . . . . . . . . . . . . . . . . . . . . GF 66

Colorado Desert........................................ W 225

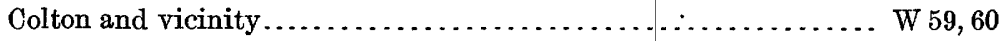

Concord quadrangle.................................. GF 193

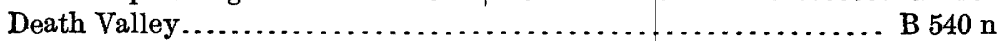


California, areas, eastern ................................... B 308

areas, foothill belt east of Los Angeles....................... W 219

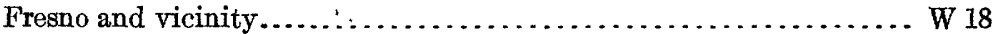

Haywards quadrangle...................................... GF 193

Indian Wells Valley....................................... 7

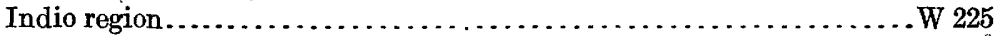

Kings River Delta..................................... W $5 \hat{8}$

Los Angeles River valley........................ W 112; A 21 rv a

Lytle Creek valley................................. A 21 Iv a

Marysville quadrangle..................................... 17

Mission Valley................................... A 21 rv a

Mohave Valley ................................. A 21 rv a

Monterey County...................................... B 264

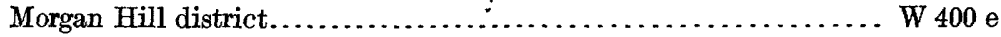

Niles Cone........................................ W 345 h

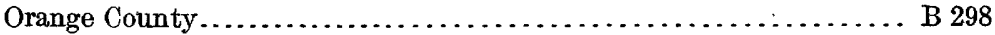

Owens Valley....................................... 181,294

Redding quadrangle.................................... GF 138

Sacramento Valley ......................................... 375 a

Salinas Valley......................................... 89

Salt Wells Valley....................................... 7

San Bernardino Valley.......................... W 59,60, 142; S 6

San Diego County............................... W 52; B 264

San Francisco quadrangle............................ GF 193

San Joaquin Valley.......................... W 222, 398; B 653

San Luis Obispo County ................................. B 298

San Luis quadrangle........................................ 101

San Mateo quadrangle.................................. GF 193

Santa Barbara and vicinity................................. 116

Santa Clara County................................... B 298

Santa Clara Valley............................... W 345 h, 400 e

Santa Cruz quadrangle................................... GF 163

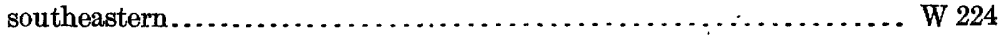

southern............................... W 146, 213, 219, 331

Tamalpais quadrangle.................................. GF 193

Truckee quadrangle.................................. GF 39

Tulare County ...................................... B 298

Ventura County . ........................................ B 298

artesian water in............ W $45,59,60,89,137,138,139,142,146,181,213$,

$219,222,224,225,278,398$; GF 138, 163, 193; S 6

auriferous gravels of, water in

GF 66

bibliography of ground water in .................W 57, 120, 142, 149, 163

cost of pumping in..................... 116, 137, 138, 139, 219, 222, 398

cost of wells in.................. W 137, 138, 139, 142, 219, 222, 225, 278, 398

evaporation in............................... 18, 58, 181, 294, $345 \mathrm{~h}$

infiltration tunnels in ....................... W 116; A 19 II b; GF 193

irrigation with ground water in .. W $17,45,58,59,60,89,116,137,138,139,142,181$, $219,222,225,278,345$ h, 375a, 398, 400 e; A 16 rre

law relating to ground water in ............................W 122, 152 mineral water in ................. W 338; B 32; MR 1883-1916; GF 39, 101 public water supplies in..................... W 59,60, 116,345 h; GF 193

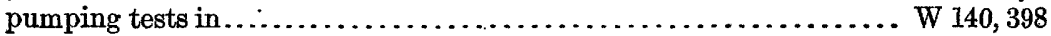


California, quality of ground water in... W 58, 59, 116, 137, 138, 139, 142, 224, 225, 278, 338,$398 ;$ B $32,529,540$ n, 606, 652; MR 1916; GF 17, 39

seepage water in. W 18, 58; A 19 II b springs in ..................... W 177, 251, 298, 300, 338, 370; B 32; GF 39 temperature of ground water in ................... W 142; B 32; GF 39

, underflow of Los Angeles River in . ........................... W 112 of Mohave Rivier in .................................... W 140 of Rio Hondo in ...................................... W 140 of San Gabriel River in............................... W 140 water table in, fluctations of.... W 58, 142, 213, 219,251, 331, $345 \mathrm{~h}, 400$ e; GF 193 watering places in ................................. W 224; B 308 well records for W 57,$149 ;$ B 264,298

California well casing. See Well casings and Well construction.

Calkins, F. C., Geology and water resources of east-central Washington...... W 118 Caloosahatchee marl, water in, in Florida.......................... W 319

Calvert, W. R., Geology of Standing Rock and Cheyenne River Indian reservations, N. Dak. - S. Dak................................... 575 B

Calvert formation, water in, in Delaware........................ GF 137 water in, in Maryland ........................ GF 136, 137, 152, GF 204

Cambrian formations, water in, in Alabama............. W 114; GF 175; S 2 water in, in Connecticut.................................. W 374 in Delaware.......................................... W 106 in Georgia............................................. $114 ; 4$ in Illinois. . . . . . . . . . . . . . W 114; A 17 I h; M 38; GF 81, 145, 200 in Iowa............................ W 114, 145, 293; GF 145, 200 in Kansas........................................... GF 148 in Maine............................................ 149, 158 in Maryland...1..................................... 179, 204

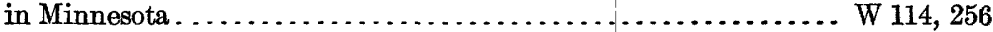
in Missouri........................... 114, 145, 195; GF 148 in Nebraska...................................... P 32 in New Mexico . . .................................... GF 199 in New York . . . . . . . . . . . . . . . . . . . . . . . . . . . . . . . . W 114 in North Carolina.............................. GF 124, 147, 151 in Ohio............................................. W 259 in Pennsylvania...................... W 106, 110, 114; GF 162, 179 in South Carolina . ..................................... GF 147 in South Dakota. W 227, 428; A $21 \mathrm{Iv} \mathrm{b;} \mathrm{P} \mathrm{32,} \mathrm{65;} \mathrm{GF} \mathrm{107,} \mathrm{127,} \mathrm{GF} \mathrm{128,} 164$ in Tennessee ................................... GF 124, 151 in Virginia.......................................... W 114 in West Virginia.................................... W 114, 178 in Wisconsin.......................... W 114, 145; GF 140, 145; S 9 in Wyoming.............A 21 rv b; P 32, 51, 65; GF 107, 127, 128, 150 See also specific formations.

Campbell, M. R., Barnesboro-Patton folio, Pa....................... GF 189

Danville folio, Ill.-Ind...................................... GF 67

Geologic structure of Punxsutawney, Curwensville, Houtzdale, Barnes-

boro, and Patten quadrangles, Pa.......................... $531 \mathrm{~d}$

Canada, ground water in, bibliography of ......................... W 163

southern Manitoba . . . ..................................... M 25

Canyon formation, water in, in Texas............................ W 317

Capacity of rocks for water. See Quantity and Porosity.

Capacity of wells. See under Wells. 
Cape Fear formation, water in, in North Carolina .................... S 5

Capillarity, fluctuation of water table in relation to................... W 155

ground water in relation to ................................... 47

water retained by................................... $19 \mathrm{~m} \mathrm{~b}$

Capillary discharge of ground water, in California ................. W 294; S 7

in Kansas................................................... 153

in Nevada . . . .................................... W $375 \mathrm{~d}, 423$

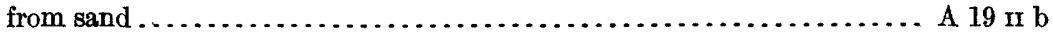

Capillary movements of ground water............................ A 19 In b

Capillary rise of ground water, height of ............... W 294, 343; A 19 II b

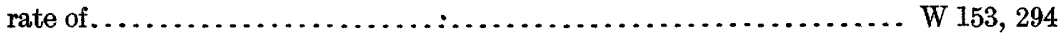

Capps, S. R., Underground waters of north-central Indiana................ W 254

Carbonates in ground water................................. 254,

$259,273,338,341,398$; В 330, 491, 616; MR 1911 Ir, 1916

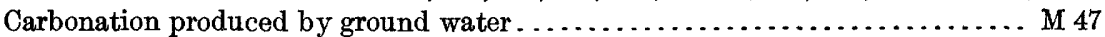

Carboniferous formations, water in, in Alabama...........W 114; GF 175; S 2

water in, in Arizona................................. W 380; B 435

in Arkansas........................ W 110, 114, 145; GF 122, 154

in Colorado. .................................... P 32,52

in Georgia. . . .................................... W 114; S 4

in Illinois... W 114; A $17 \mathrm{II} \mathrm{h;} \mathrm{B} \mathrm{438,} \mathrm{506;} \mathrm{M} \mathrm{38;} \mathrm{GF} \mathrm{105,} \mathrm{185,} \mathrm{188,} \mathrm{195;} \mathrm{S} 3$

in Indiana...................... W 26, 114, 254; A $18 \mathrm{rv}$ b; GF 105

in Iowa............................................ W 114, 293

in Kansas..................... W 273; P 32; B 238; GF 148, 159, 206

in Kentucky............................... W 114; GF 184, 233

in Maryland............................. W 110, 114; GF 160, 179

in Michigan . . ........................... W 30, 31, 114, 182-183

in Mississippi. ........................................ W 114

in Missouri...................... W 114, 195; B 438; GF 148, 206

in Montana. ......................................... 221

in Nebraska.......................... W 12; A 19 rv c; P 17, 32

in New Mexico............................ W 123, 158, 343; B 435

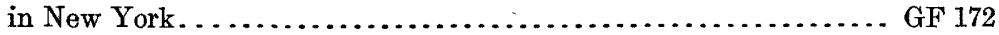

in Ohio.................... W 91, 114; A $18 \mathrm{rv} \mathrm{b,} 19 \mathrm{IV}$ b; GF 184

in Oklahoma................... W 148; B 641 b, 691c; GF 122, 132, 154

in Pennsylvania . . . ............................... W 110 ,

114; B 300, 531 d; GF 102, 121, 123, 133, 144, 146, 160, 172, 174, 189

in South Dakota..... W 227, 428; A 21 Iv b; P 32, 65; GF 107, 127, 128, 164

in Tennessee........................................W 114

in Texas . . .................W 154, 191, 276, 317; A 18 Ir b; GF 194

in Utah............................................. 380

in Virginia.............................................. 114

in West Virginia....................... W 110, 114; GF 160, 179, 184

in Wyoming................................. 21 rv b;

P $32,51,53,65 ;$ B 364; GF 107, 127, 128, 141, 150, 173

See also specific formations.

Carpenter, Everett, Ground water in Boxelder and Tooele counties, Utah. ... W 333

Ground water in southeastern Nevada . . . . . . . . . . . . . . . . . W 365 Casings in wells. See Well casings.

Casper formation, water in, in Wyoming ................. B 364; GF 173

Castle Hayne formation, water in, in North Carolina . . . . . . . . . . . . . . S 5

Catahoula formation, water in, in Arkansas........................ P 46

water in, in Louisiana............................... W 114; P 46

in Texas............................................ 335 
Catchment areas. See Origin of ground water and Absorption of water.

Caverns, deposits made by ground water in.............. W 233, 258; A 18 In b

formed by ground water, in Florida......................... W 319

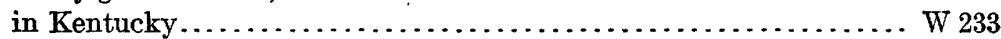

water in................. W 114, 233, 255, 258, 319; A 18 Iv b, 21 Iv c; S 3

pollution of.................................... 233,258

See also Pollution of water and Sink holes.

Caves. See Caverns and Sink holes.

Cellar's, drainage of, into wells.

W 258

Cementation produced by ground water......................... M 47

Chadron sandstone, water in, in Colorado...................... P 32 water in, in Nebraska.

A 19 Iv; P 17, 32; GF 156

in South Dakota............................... W 227; P 32

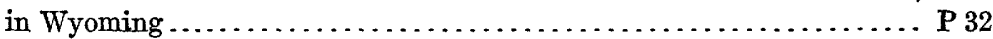

Chalk, water in, in South Dakota........................ $10.113,114,156$

Chalybeate waters. See Quality of ground water in regard to iron.

Chamberlin, T. C., Requisite and qualifying conditions of artesian wells...... A s c

Chambers, A. A., Comparison of American and European mineral waters.. MR 1916

Chandler, A. E., Water storage on Cache Creek, Cal.................... W 45

Chanute shale, water in, in Kansas.............................. B 238

Character of ground water. See Analyses, Pollution, and Quality.

Chattahoochee formation, water in, in Florida .................... W 319 water in, in Georgia............................................ 341

Chemistry of ground water. See Analyses; Analysis, Pollution, Purification,

and Quality.

Cherokee shale, water in, in Missouri

$\mathrm{W} 195$

Chesapeake group, water in, in Atlantic Coastal Plain ............... B 138

water in, in Maryland .................................... 23, 204

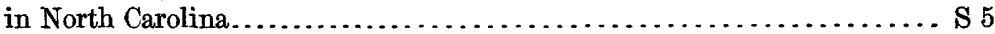

in Virginia................................... 114; GF 23 ; S 8

Chester group, water in, in Illinois ...................... W 114; B 438

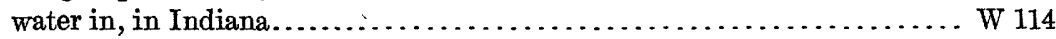

in Missouri......................................... $195 ; \mathrm{B} 438$

Chester Valley limestone, water in, in New Jersey ................. W 106

water in, in Delaware...................................... W 106

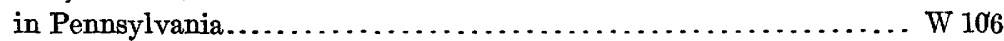

Chickamauga limestone in Georgia.................................. S 4

Chickies quartzite, water in, in Delaware......................... W 106

water in, in New Jersey................................. W 106

in Pennsylvania.................................. 106; GF 162

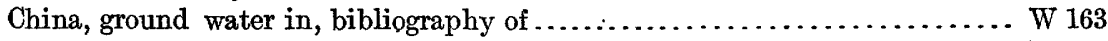
well drilling in .......................................... W 257

Chlorine in ground water. See Analyses, Analysis, Pollution, and Quality.

Chlorine, normal, in ground water, in Connecticut............... W 144, 232

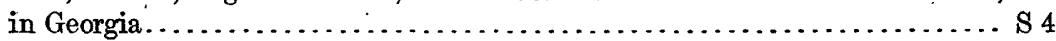

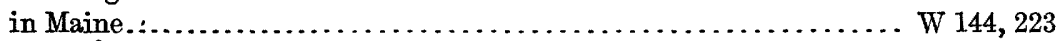

in Massachusetts....................................... 114, 144

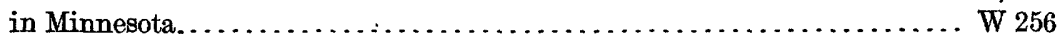

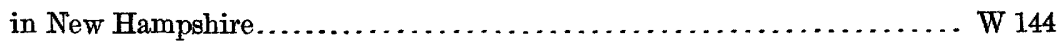

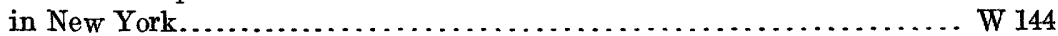

in Ohio................................................. 259

in Rhode Island.......................................... 114, 144

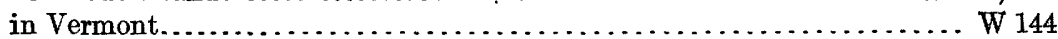

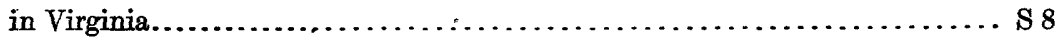


Choctawhatchee marl, water in, in Florida....................... W 319

Chouteau limestone, water in, in Missouri......................... W 195

Chugwater formation, water in, in Wyoming .............. B 364, 656; GF 173

Cincinnati shale, ground water in relation to, in Wisconsin............... S 9

Cincinnatian formations, water in, in Indiana....................... W 254

Circulation of ground water. See Movements of ground water.

Cisco formation, water in, in Texas.............................. W 317

Cisterns, catchment areas for........................... A 14 Ir a

combined with wells.................................... 255

construction of.............................. W 255; A 14 rn a

effect of "creep" on................................. A 14 II a

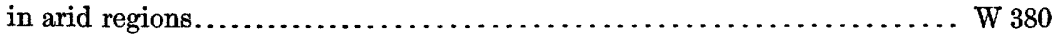

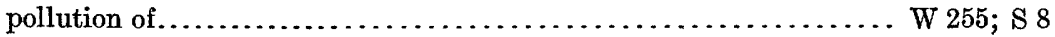

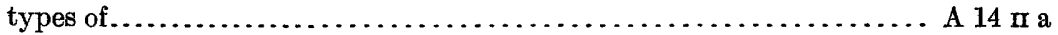

City water supplies. See Public water supplies.

Clack valves in pumps........................................ 14

Claiborne formation, water in, in Arkansas................... W 399; P 46

water in, in Georgia ................................... 341

in Louisiana...................................... P 46

in Mississippi......................................... 158

Clapp, F. G., Amity folio, Pa................................ GF 144

Barneshoro-Patton folio, Pa.............................. GF 189

Bibliography of ground-water literature in $1905 \ldots \ldots \ldots \ldots \ldots \ldots \ldots \ldots$ W 163

Composition of mineral springs in Maine......................... W 258

Economic geology of the Amity quadrangle, $\mathrm{Pa} \ldots \ldots \ldots \ldots \ldots \ldots \ldots \ldots$ B 300

Patoka folio, Ind.-Ill..................................... 105

Rogersville folio, Pa.......................................... 146

Underground waters near Manassas, Va....................... 258

Underground waters of southern Maine ....................... W 223

Underground waters of southwestern Ohio...................... W 259

Water resources of the Curwensville, Patton, Ebensburg, and Barnesboro

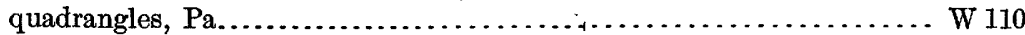

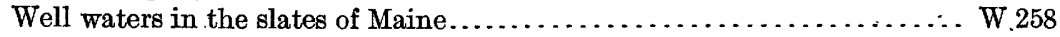

Clark, W. B., Coastal Plain of North Carolina......................... S 5

Philadelphia folio, Pa.-N. J.-Del ......................... GF 161

Trenton folio, N. J.-Pa.................................. GF 167

Clark, W. O., Ground-water resources of the Niles cone area, Cal.......... W $345 \mathrm{~h}$

Ground water for iprigation in the Morgan Hill area, Cal............W $400 \mathrm{e}$

Clarke, F. W., Data of geochemistry ......................... B 330,491, 616

Water analyses. . . . . . . . . . . . . . . . . . . . . . . . . . . W 364

Classification of ground water, chemical.. W $254,273,274,341,398,399 ; \mathrm{B} 479,606$; S2

of ground water, criteria for ........................ B 330,491, 616

for boiler use . . . . . . . . . . . . . W 254, 259,274, 293, 335, 341, 398, 399

for domestic use . . . . . . . . . . . . . . . . . . . W 335, 341, 343, 398, 399

for irrigation . . . . . . . . . . . . . . . . . . . . . $274,333,335,398,399$

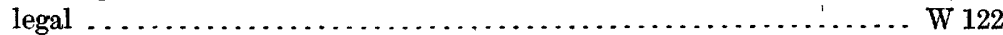

numerical standards for . . . . . . . . . . . . . . . . . . 274,398

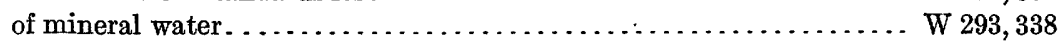

of oil-field waters. . . . . . . . . . . . . . . . . . . . . . . . . 653

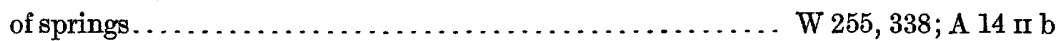

of well casings. . ............................................... W 255

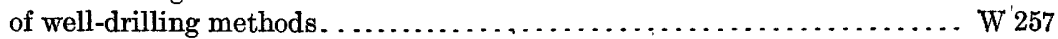

of wells. . . . . . . . . . . . . . . . . . . . . 255

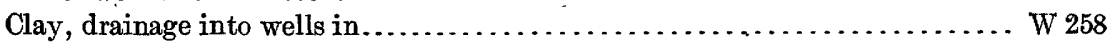

water in .................. W 114, 223, 255, 257, 319, 425 b; A 21 vII 
Cleaning of wells. See Well construction.

Clear Fork formation, water in, in Texas.

Cleavage in relation to ground water.

Cleavage planes, water in

W 114, 223; B 319

Climate. See Evaporation and Precipitation.

Clinton limestone, water in, in Indiana...

water in, in Michigan.

in Ohio.

W 114,$259 ;$ A 19 Iv b

Clinton sandstone, water in, in Michigan $\mathrm{W} 30$ water in, in Ohio.

Cloverly sandstone, water in, in Wyoming. P 51, 53; B 364; GF 141, 142, 173 See also Dakota sandstone.

Coal beds, water in, in Pennsylvania. B 300, 447; GF 174 water in, in South Dakota . . . . . . . . . . . . . . . . . . . . . . . B 627

Coal-mine waters, fish killed by .................................. 273 sulphuric acid in . . . . . . . . . . . . . . . . . . . . . . . 273

Coastal Plain. See individual States.

Cockfield formation, water in, in Arkansas................... 114;P46 water in, in Louisiana. ................................ $114 ; \mathrm{P} 46$

Coefficient of kiscosity ............................ W 67; A 19 II b

Cold water shale, water in, in Michigan..................... 30,114

Coli bacillus in ground water......................... W 193, 256, 315

Collecting areas. See Absorption and Origin.

Collecting galleries. See Infiltration ditches and tunnels.

Color in ground water........................ W 240, 254, 259, 338, 341,398

Colorado, areas, Apishapa quadrangle....................... GF 186 areas, Arkansas Valley.......................... A 17 In f; P 52

Boulder district. . . . . . . . . . . . . . . . . . . . . . . . . . . 265

Castle Rock quadrangle................................. GF 198

Colorado Springs quadrangle. ...................... GF 203

De Beque oil field ............................... B $531 \mathrm{c}$

Denver Basin............................... M $27 ; \mathbf{P} 32$

eastern........................ A $17 \mathrm{mf}, 21 \mathrm{rv} \mathrm{c,} 22 \mathrm{rv} \mathrm{c} ; \mathrm{P} 32,52$

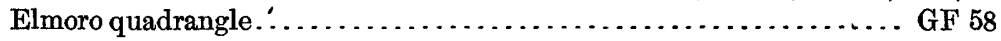

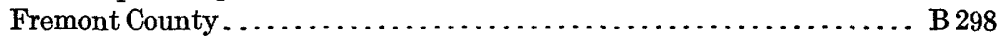

Garfield County............................. B 531 c

Greeley and vicinity.$\ldots \ldots \ldots \ldots \ldots \ldots \ldots \ldots \ldots \ldots \ldots \ldots \ldots \ldots \ldots \ldots \ldots \ldots$

Mesa County ................................... 531 c

Nepesta quadrangle.................................... GF 135

northeastern . . . . . . . . . . . . . . . . . . 16 nf

northwestern. . . . . . . . . . . . . . . . 350

Ouray quadrangle................................ 153

Pueblo County. . . . . . . . . . . . . . . . . . . . . . . . . B 298

Pueblo quadrangle................................... GF 36

Rangely oil district: . . . . . . . . . . . . . . . . . . . . . . B 350

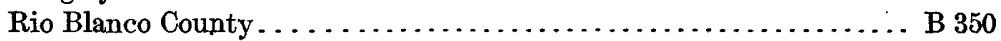

San Luis Valley . . . . . . . . . . . . . . . . . . . . . 240

Silverton quadrangle............................. GF 120

South Platte Valley .............................. 184

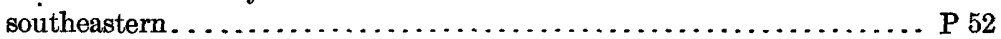

Spanish Peaks quadrangle............................... GF 71

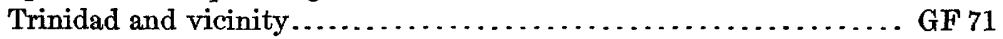

Walsenburg quadrangle.............................. GF 68 artesian water in .......... W 9; A 16 II f, 17 II f, 21 Iv a $;$ M 27; P 32, 52; B 265, 
Colorado, bibliography of ground water in............. W 57, 120, 149, 163

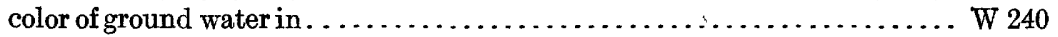
early irrigation with ground water in.$\ldots \ldots \ldots \ldots \ldots \ldots \ldots \ldots \ldots \ldots$ A 16 II e flowing well at Alamosa ............................... 21 Iv a irrigation with ground water in ........ W 5, 9, 240; A 16 II ef, 21 Iv c, 22 Iv c law relating to ground water in .......................... 9,122 mine water in ................................... 364 ; GF 120 mineral waters in .................... B 32; MR 1883-1916; GF 153, 203 public water suppliesin...................... W 240;P 32;GF 198 pumping in, cost of ......................................... 9 quality of ground water in .............. W 9, 240, 364; A 17 II f; M 27; P 32, 52; MR 1916; GF 135, 153, 203

salt water in B $531 \mathrm{c}$ seepage in ............................... $9,50,358$

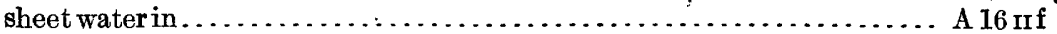

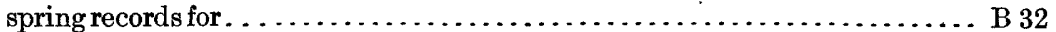

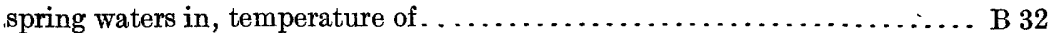

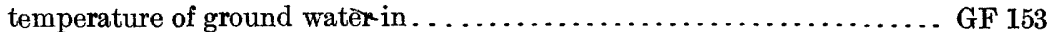

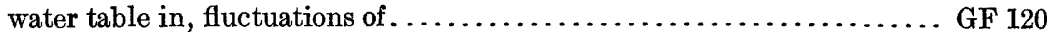

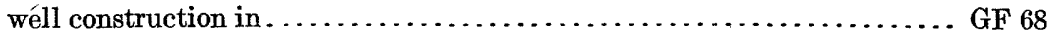
well records for ........................... W 57, 149;B 131, 264, 298 wells in, cost of ..................................... GF 68 Colorado group. See specific formations.

Colorado River basin, bibliography of ground water in ................ W 340 Columbia clay, water in, in Missouri............................ W 195 Columbia formation, water in, in Atlantic Coastal Plain................. B 138

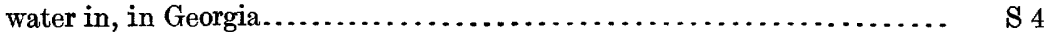

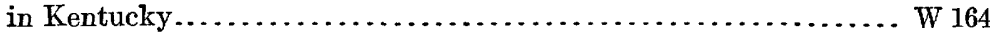
in Maryland....................................... 13, 23 in Tennessee........................................... 164 in Virginia..................................... 13, 23; 8 Columbia River lava, water in, in Washington and Oregon. See Lava.

Comanche series, water in, in Texas.............................. W 317 Composition of ground water. See Analyses, Analysis, and Quality.

Condra, G. E., Geology and water resources of Missouri River valley in Nebraska. W 215 Geology and water resources of Republican River valley, Nebr......... W 216 Confining beds for artesian water ........................... B 319; A $5 \mathrm{c}$ Conglomerate, drainage into wells in ........................... W 258 water in..................................... $110,232,255,257$ Connate water........ W 160, 398; P 90 h; B 319, 330,491, 530 b, 616, 653, 661 d; S 5 Connecticut, areas, all of State......................... 106, 114, 160, 232 areas, Bradford Point town................................. W 232

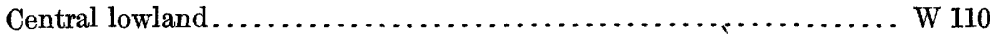
Hartford district..................................... W 374 near New York...................................... P 44 North Haven town..................................... W 232

Salisbury district...................................... W 374

Saybrook district.................................... W 374

Stamford district.................................... W 374

Warren town.......................................... W 232

Waterbury district..................................... W 397

Willimantic district................................... W 374 artesian water in................................ W 232, 374, 397 
Connecticut, bibliography of ground water in............... W 114, 120, 163 infiltration tunnels in................................. 374,397 isochlors in ......................................... 144, 232 law relating to ground water in ............................... 152 mineral water in....................... W 114, 232; B 32; MR 1883-1916 public water supplies in . .......................... W 232,374, 397 quality of ground water in......... W 102, 110, 144, 232, 364, 374, 397; B 32; P 44 spring records for......................................... B 32

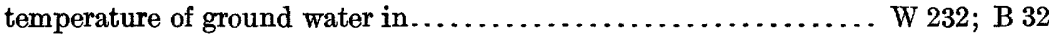

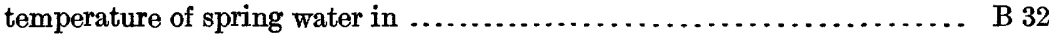
well records for........................ W 57, 149, 274, 397; B 264, 298 wells in, cost of .......................................... 160 Conservation of artesian water................................. 142 , $145,158,219,225,234,256,278,293,400$ b, 423,425 a; $\mathrm{S} 8$ Conservation of ground water in United States..................... W 234 Construction of wells. See Well construction.

Contact planes, artesian water in................................. B 319 Contamination. See Analyses, Pollution, Purification, and Quality.

Contours of water table. See under Maps.

Cook Mountain formation, water in, in Texas...................... W $375 \mathrm{~g}$ Cooper, W. F., Well and spring records in lower Michigan................ W 102 Copper in ground water.................................. W 399; B 47 Corniferous limestone, water in, in Ohio .................. W 114; A 19 Iv b Corrosion by water. See under Quality and Well screens.

in boilers, methods of calculating.

Cost. See under specific subjects.

Cotton manufacturing, quality of water for.................... 254, 298

Couche, definition of ...................................... A 14 In a

Court decisions. See Law relating to ground water.

Cowgill, E. B., Irrigation on the Great Plains...................... W 5

Crane, W. R., Economic geology of Iola quadrangle, Kans.............. B 238

Crenothrix in ground water.............................. 254, 259, 338

Cretaceous formations, water in, in Alabama....................W 114; S 2 water in, in Arizona............................... W 380; B 435 in Arkansas.................................. 114, 399; P 46 in Colorado............................ 17 I f; M 27; P 32, 52; B 265,350 ; GF $36,58,68,71,135,153,186,203$ in Delaware..................... W 106, 114; B 138; GF 137, 162 in District of Columbia.................... B 138; W 114; GF 70, 152 in Georgia......................... 6 67, 114, 341; B 138, 164; S 4 in Iowa................................ W 114, 293; GF 156 in Kansas................................... W 6, 273; P 32 in Kentucky....................................... 114, 164 in Louisiana............................................. W 114 in Manitoba........................................... 25 in Maryland...................W 114; B 138; GF 13, 137, 152, 182, 204 in Minnesota.......................... W 114, 256; M 25; GF 117 in Mississippi...................................... W 114, 159 in Montana...................................... G5 55, 128 in Nebraska... W 12, 70, 215, $425 \mathrm{~b} ; \mathrm{A} 19 \mathrm{IV}$ c; P 17, 32; GF 85, 87, 88, 108, 156 in New Jersey................. W 106, 114; B 138; GF 137, 157, 162, 167 in New Mexico....................... W 123, 343, 380; B 435; GF 199 in New York . . . . . . . . . . . . . . . . . . W 114; P 44; B.138; GF 157 
Cretaceous formations, water in, in North Carolina...... W 114; B 138; GF 80; S 5 in North Dakota.................... M 25; B 575; GF 117, 168, 181

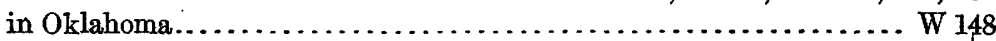
in Pennsylvania........................ W 106; B 138; GF 162, 167 in South Carolina .........................W 114; P $90 \mathrm{~h} ; \mathrm{B} 138$ in South Dakota........ W 34, 90, 227, 428; A 17 Ir g, A 21 Iv b; P 32, 65; B 575,627 ; GF $85,96,97,100,107,108,113,114,127,128,156,164,165$ in Tennessee.................................... W 114, 164 in Texas.. W 190, 191, 276, 317, 335, $375 \mathrm{~g} ;$ A 18 II b, 21 vi; GF 42, 64, 76, 183

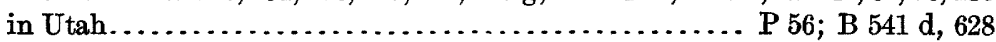
in Virginia . . . . . . . . . . . . . . . . . . . 114; B 138; GF 13, 80; S 8 in Wyoming .............. W 70,425 b; A 21 rv b; P 32, 51, 53, 56, 65; B 364, 471, 543, 621 1, 641, 656; GF 107, 127, 128, 141, 142, 150, 173 See also specific formations.

Crevices. See under Caverns.

Crider, A. F., Drainage by wells in Arkansas.................... W 160

Geology and ground waters of northeastern Arkansas.............. W 399

Underground-water resources of Mississippi ................... W 159

Criteria for locating ground water . . . . . . . . . . . . . . . W 224, 423; B 308

Crooked wells............................................... 257

Crosby, W. O., Sizing and filtration tests on Long Island, N. Y . . . . . . . P P 44

Water from delta type of sand plain ......................... 145

Water resources of Massachusetts and Rhode Island................. W 114

Well and spring records in Massachusetts and Rhode Island .......... W 102

Cross, Whitman, Geology of the Denver Basin, Coló................ M 27

Ouray folio, Colo........................................... 153

Silverton folio, Colo . . . . . . . . . . . . . . . . . . . . . . . . . . . . . . GF 120

Crystalline rocks, artesian water in ............. W 160, 232,374, 397; B 391 deep wells in . . . . . . . . . . . . . . . . . . . . . . . . . 8

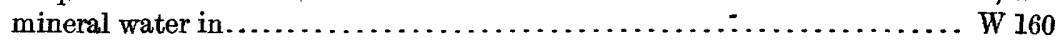
water in .......................... 160, 232; B 319; A 5 c, 19 In b bibliography of ...................................... 232

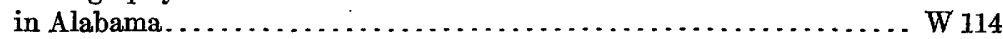
in California ...................................... 219,338 in Connecticut............................ $232,374,397$ in District of Columbia ........................... 114

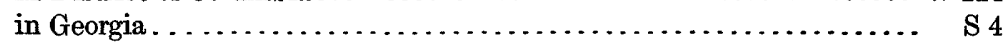
in Maine..................................... 145, 223; GF 149, 158 in Maryland......................................... 152, 204

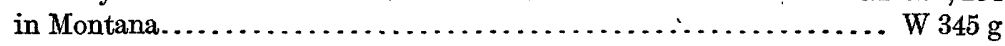
in Nevada......................................... 365 in New Jersey ........................ W 114; GF 157, 167, 191 in New Mexico........................................ W 343 in New York................................ 114; GF 157 in North Carolina. . . ........................ GF 124, 147, 151; S 5 in Pennsylvania................................ 106, 114, 167 in South Carolina......................................... 147 in Tennessee................................... 124,151

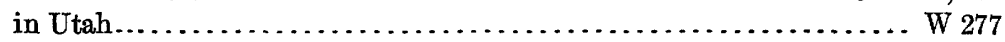
in Virginia....................................... 114 ; 8

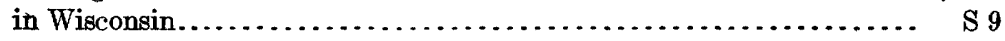

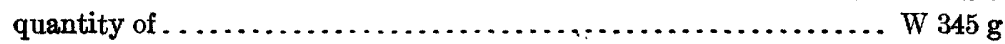
wells in, yield of $. ., \ldots, \ldots, \ldots, \ldots, \ldots, \ldots, \ldots, \ldots, \ldots, \ldots, \ldots, \ldots, W 232$ 
Cuba, ground water in $\mathrm{W} 110$

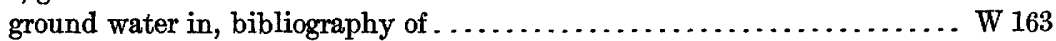

Cumings, E. R., Columbus folio, Ohio ........................ GF 197

Curbs. See under Wells and Well casings.

Cylinder pumps.

D.

Dakota sandstone, water in

W 67 water in, in Colorado.

W $9 ;$ A 16 Ir f, 17 In f;

M 27; P 32, 52; B 265; GF 36, 58, 68, 71, 135, 18

in Towa. W 215, 293

in Kansas. W 6,273; A 16 Ir f, 21 rv c; P 32 in Montana GF 128

in Nebraska

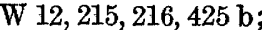

A 16 II $\mathrm{f}, 21$ Iv c; P 32; GF 85, 87, 88, 108 in North Dakota A 17 II $\mathrm{g} ;$ M 25 ; B 575; GF 168, 181 in Oklahoma. A 21 IV c in South Dakota . . . . . . . W 34, 90, 215, 227, 428; A $17 \mathrm{Ir} \mathrm{g,} 21 \mathrm{rv} \mathrm{b}$; P 32, 65; B 575; GF 85, 96, 97, 99, 100, 107, 108, 113, 114, 128, 164, 165

in Texas W 191

in Utah B 541, 628 in Wyoming. W $425 \mathrm{~b} ; A 21 \mathrm{rv} \mathrm{b}, 21 \mathrm{rv} \mathrm{c}$; P $32,51,53,65 ;$ B 364, 471 a; GF 107, 127, 128, 141, 142, 173

Dale, T. N., Fort Ticonderoga quadrangle, Vt.-N. Y . . ............... W 110 Dams, fluctuation of water table in relation to.................W 155; P 44 Dams for ground water, artificial................. W 39, 67, 199; A 13 III b

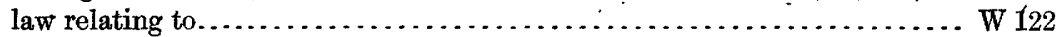
natural......................................... $320,343,423$

Darcy, on flow of water through soils..................... W 67; A 19 In b Darton, N. H., Aladdin folio, Wyo.-S. Dak.-Mont.................... GF 128 Artesian water in the vicinity of the Black Hills, S. Dak............ W 428 Artesian water of a portion of the Dakotas..................... A $17 \mathrm{II} g$ Artesian wells on Atlantic Coastal Plain....................... B 138 Bald Mountain-Dayton folio, Wyo.......................... GF 141 Belle Fourche folio, S. Dak................................. GF 164 Camp Clark folio, Nebr.................................... GF 87 Cloud Peak-Fort McKinney folio, Wyo........................ GF 142 Deep borings in the United States, Part 1.................... W 57 Deep borings in the United States, Part $2 \ldots \ldots \ldots \ldots \ldots \ldots \ldots \ldots \ldots . \ldots \ldots$ W 61 Deep borings in the United States............................ W 149 Deming folio, N. Mex ..................................... 207 Devils Tower folio, Wyo.................................... GF 150 Edgemont folio, S. Dak.-Nebr............................. GF 108 Fredericksburg folio, Va.-Md.............................. 13 Geology and mineral resources of the Laramie Basin, Wyo............ B 364 Geology and underground waters of Arkansas Valley in eastern Colorado.. P 52 Geology and underground waters of the central Great Plains............. P 32 Geology and underground waters of Luna County, N. Mex....... W 345 c; B 618 Geology and underground waters of Nebraska west of the 103d meri-

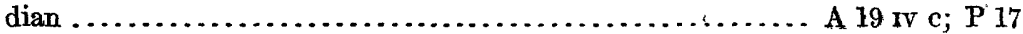

Geology and underground waters of South Dakota................ W 227 Geology and water resources of the Black Hills and adjoining regions,

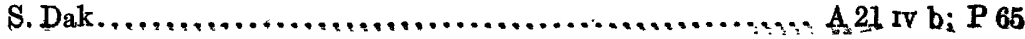


Darton, N. H., Geology of the Bighorn Mountains, Wyo............... P 51 Laramie-Sherman folio, Wyo............................... GF 173

Newcastle folio, Wyo.-S. Dak............................. GF 107

New York City folio..................................... GF 83

Nomini folio, Md.-Va.................................. GF 23

Norfolk folio, Va.-N. C..................................... GF 80

Oelrichs folio, S. Dak.-Nebr............................. GF 85

Passaic folio, N. J.-N. Y.................................... 157

Philadelphia folio, Pa.-N. J.-Del. ........................ GF 162

Reconnaissance of northwestern New Mexico and northern Arizona..... B 435

Scotts Bluff folio, Nebr.................................. GF 88

Silver City folio, N. Mex.................................... GF 199

Sundance folio, Wyo.-S. Dak................................. 127

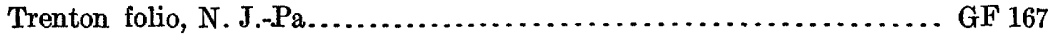

* Underground waters of Delaware, District of Columbia, Maryland, and

Virginia............................................. W 114

Underground waters of southeastern Nebraska.................... W 12

Washington folio, D. C.-Md.-Va............................... GF 70

Well boring and irrigation in eastern South Dakota in $1896 \ldots \ldots \ldots \ldots$ A 18 IV c

Davis, A. P., Irrigation near Phoenix, Ariz........................ W 2

Davis, C. A., Wells and municipal water supplies in the southern peninsula of

Michigan.......................................... 182, 183

Dawson arkose, water in, in Colorado.............................. GF 198

Deadwood sandstone, water in, in South Dakota................. W 227, 428;

A $21 \mathrm{Iv} \mathrm{b;} \mathrm{P} \mathrm{32,65;} \mathrm{GF} \mathrm{107,} \mathrm{128,} 164$

water in, in Wyoming.......... A $21 \mathrm{rv} \mathrm{b}$; P 32, 51,65; GF 107, 128, 141, 142, 150

Dean, H. J., Water resources of California, Part III.................... W 300

Débris-filled basins, fluctuations of water table in .................. W 142,

$219,343,345 \mathrm{~g}, 345 \mathrm{~h}, 375 \mathrm{a}, 400 \mathrm{e}, 423 ; \mathrm{S} 6$

ground water in, discharge of .................. W 142, 219, 294, 423; S 6,7

early description of ................................. A 13 III a

in Arizona....................... W 104, 136, 320, $375 \mathrm{~b}, 425 \mathrm{a} ; \mathrm{B} 352$

in California.. W 89, 142, 219, 222, 225, 278, 294, $375 \mathrm{a}, 398,345 \mathrm{~h}, 400$; GF 163

in Colorado.............................................W 240

in Montana...................................W $345 \mathrm{~g} ; 400 \mathrm{~b}$

in Nevada........................... W 365, 375 d, 423; B 530 r

in New Mexico............... W 123, 260, 275, 343, 345 c; B 618; GF 207,

in Oregon........................................ W 220,231

in Texas...................................... 166, 194; S 1

in Utah............................... W 157, 199,217,277, 333

quantity of ............................ 142, 219, 294,423; S 6,7

See also Valley fill.

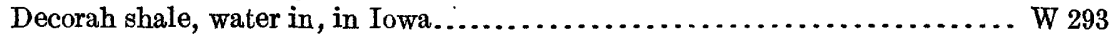

water in, in Minnesota....................................... W 256

Deep wells.................................... W 57, 61, 149, 257

Deflection of wells, methods of measuring........................ W 257

Deforestation, fluctuation of water table in relation to................ W 155

Delaware, areas, all of State................................... W 114

areas, Coastal Plain......................................... 138

Dover quadrangle.................................. GF 137

New Castle County..................................... B 298

northeastern................................... 106; GF 162

artesian water in................................ B 138; GF 137 
Delaware, bibliography of ground water in............. W 114, 120, 149, 163 mineral water in ............................ B 32; MR 1883-1916 public water supplies in ................................. B 138

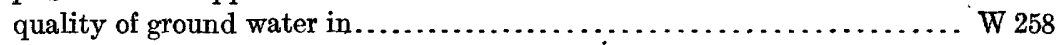

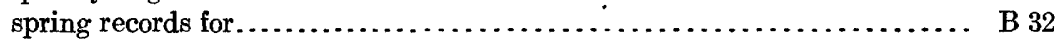
spring waters in, temperature of $\ldots \ldots \ldots \ldots \ldots \ldots \ldots \ldots \ldots \ldots \ldots \ldots \ldots \ldots \ldots \ldots$ well records for .................................. W 57, 149; B 298

Delta deposits, water in, in California.......................... 225 water in, in New England.................................... W 145

Denmark, bibliography of ground water in........................ 163

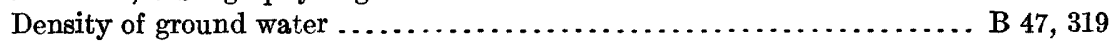

Denver formation, water in, in Colorado........................ 27

Deoxidation produced by ground water............................ 47

Depth of wells, methods of measuring.......................... 257

Depth to ground water, methods of estimating..................... W 423

Depth to which ground water penetrates............. W 67, 160; A 19 II b; M 47

Descartes on origin of ground water...........................A 14 II b

Desert basins. See Débris-filled basins and Valley fill.

Desert flats, ground water under.........................W 277; B $530 \mathrm{k}$

Desert springs. See Watering places and Springs.

Desert watering places. See Watering places.

Desert wells. See Watering places and Wells.

De Smet formation, water in, in Wyoming................ P 51; GF 141, 142

Des Moines group, water in, in Iowa ............................... W 293 water in, in Missouri........................................ 195

Deussen, Alexander, Geology and underground waters of the Texas Coastal Plain.................................................. W 335 Ground water in Lasalle and McMullen counties, Tex ............. W $375 \mathrm{~g}$

Development of wells by gravel screens......... W 255, 256, 293, 343, 345a, 345g by pumping. . . . . . . . . . . . . . . $\ldots \ldots \ldots \ldots$ W $255,256,293,343,345 \mathrm{a}, 345 \mathrm{~g}$ by rescreening. . . . . . . . . . . . . . . . . . . . . . . . . . 255

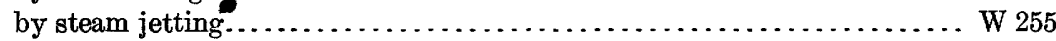
by use of exposures................................ 255,257 See also Well construction.

Devonian formations, water in, in Alabama............... W 114; S 2 water in, in Arkansas . . . . . . . . . . . . . . . . . . . . . . . . . . . W 114

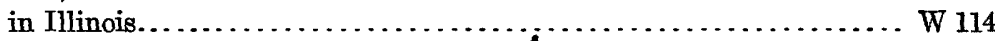
in Indiana.................................. $26,114,254 ;$ A 18 Iv b in Iowa ....................................... 114, 145, 293 in Kentucky.................................... 114, 233 in Maryland............................. 110, 114; GF 179 in Michigan...................... W 30, 31, 114, 182, 183; GF 205 in Minnesota.......................................... W 256 in Missouri........................................ 114, 195

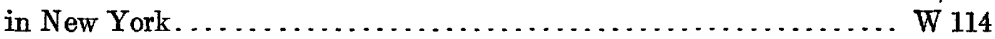
in Ohio................... W 91, 114; A 18 IV b, 19 IV b; GF 197 in Pennsylvania.......................... W 110, 114; GF 179 in Tennessee.......................................... W 114

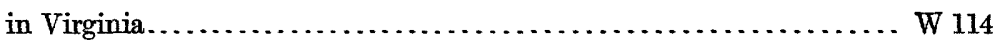

in West Virginia.......................... W 110, 114; GF 179 See also specific formations.

Dewitt formation, water in, in Texas. .............................. W 335

Diameter of soil grains, methods of measuring................. A 19 Ir b $56122^{\circ}-18-w$ SP $427-6$ 
Diesem, H. C., Cost of pumping for irrigation in western Nebraska...... W $425 \mathrm{~b}$ Diffusion of gases in ground water............................. M 47

Diffusion of solids in ground watex............................. 47

Diller, J. S., Redding folio, Cal................................ GF 138

Dip of strata, artesian conditions in relation to. . . . . . . . . . . $5 \ldots \ldots \ldots \ldots$ A

Discharge of flowing wells, dome method of measuring...... W 67, 110, 157, 219, 240

Discharge of ground water, by capillarity....................... W 275 ,

294, $345 \mathrm{~h}, 423 ; \mathrm{A} 13 \mathrm{mI}$ a, $19 \mathrm{II}$ b; S 7

by evaporation.... W 7, 153,275, 294,343,345 h, 380,423; A 13 III a, 19 II b; S 7 by seepage. See Seepage.

by transpiration................... W 7, 153,294, 320,343, $375 \mathrm{~d}, 423$; $\mathrm{S} 7$

water table in relation to....................... $153,294,343,423$

See also Capillarity, Evaporation, Quantity of ground water, Transpira-

tion, Springs, and Water table.

Discharge of wells, deceptive methods of measuring.

dome method of measuring...................... W $67,110,157,219,240$

simple methods of measuring............................ W 257

weirs for measuring............................... $150,200,320$

portable.......................................... 320

See also Wells and Artesian wells.

Disposal of ground water. See Discharge, Evaporation, Quantity, Springs, and

Transpiration.

Dissociation of solids dissolved in ground water, theory of

Dissolved solids in ground water. See Analyses and Quality.

Distilling, quality of water for.......................... W 233, 254, 398

District of Columbia, artesian water in................... 138; GF 70, 152

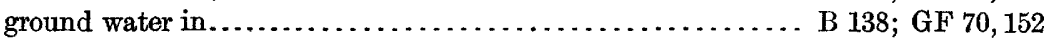

bibliography of ........................... W 114, 120,163

quality of.............................. W $364 ;$ B 32,138

mineral water in......................... W 114; B 32; MR 1896-1914

spring records for.t................................... B 32

spring water in, temperature of.......................... B 32

well records for........................................ 557,149

"Divining rod"'........................... W 255, 278, 416; MR 1882 a

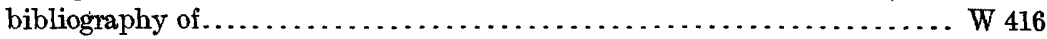

fallacy of............................. 255, 278,416; MR 1882 a

history of................................ W 416; MR 1882 a

Dodge, R. E., New York City folio........................... GF 83

Dole, R. B., Chemical character of the waters in north-central Indiana...... . W 254

Chemical character of the waters in northeastern Arkansas............. W 399

Chemieal character of the waters in southwestern Ohio............... W 259

Concentration of mineral water in relation to therapeutic activity. MR 1911 II n

Fluorescein in the study of ground water....................... W 160

Ground water in Lasalle and McMullen counties, Tex............. W $375 \mathrm{~g}$

Ground water in San Joaquin Valley, Cal..................... W 398

Historical sketch of the mineral-water trade..................... MR 1914

Production of mineral waters. ............................ MR 1913-1915

Published analyses of American mineral waters............... MR $1913 \mathrm{In} \mathrm{h}$

Quality of surface waters east of the one hundredth meridian......... W 236

Quality of surface waters in Minnesota...................... W 193

Quality of the underground waters in the Coastal Plain of Georgia....... W W 341

Radioactivity of mineral waters......................... MR $1913 \mathrm{~m} \mathrm{~h}$

Salines in Silver Peak Markh, Nev ......................... B 530 r

Water supply of Savannah, Ga............................ S 11 
"Domes' ' in relation to salt water. ............................. W 335

Doons for lifting water.......................................... 1

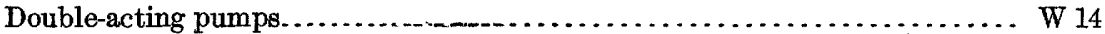

Double Mountain formation, water in, in Texas.................... W 317

Douglas formation, water in, in Kansas.......................... GF 206 water in, in Missouri...................................... 206

"Dowsing." See "Divining rod."

Drainage, effect of, on ground water........................... W 67

Drainage into wells..................................... 145,258

from cellars. . . . . . . . . . . . . . . . . . . . . . . . . . . . . . 258

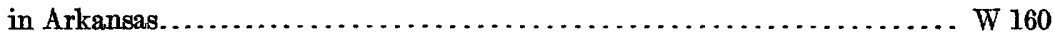

in Georgia................................................... 160

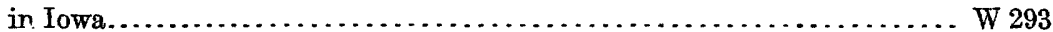

ir Michigan.............................................. 145,160

in Minnesota.............................................. W 256

Drainage of industrial wastes into wells....................... W 258

Drainage of sewage into wells................................. W 258

Drainage wells, diagrams illustrating............................ W 145

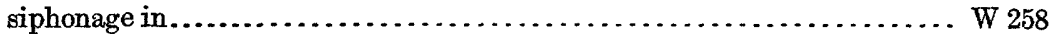

Drainage with windmills in Holland ............................ W 20

Drake, N. F., Nampa folio, Idaho-Oreg....................... GF 103

Silver City folio, Idaho.................................. GF 104

Dresbach sandstone, water in, in Iowa........................... W 293

water in, in Minnesota .............................. W 256;GF 201

See also Cambrian sandstone and Potsdam sandstone.

Drift. See Glacial drift.

Drilling. See Well construction.

Drillings from wells. See Well drillings.

Dry farming, ground water in relation to............... W 275, 277, 320, 425 a

"Dry sands" at deep horizons.............................. B 653,661d

Dundee limestone, water in, in Michigan ................. W 30, 114; GF 205

Dune sand, water in, in Arkansas:............................... W 145

water in, in Colorado............................... A 17 In f P 52

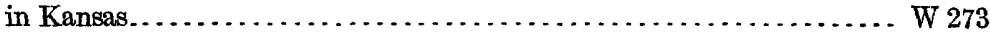

in Minnesota.................................... 256; GF 201

Dyeing, quality of water for ........................... 254, 341, 398

Dynamite, methods of using in wells...................... W 223, 255, 257

Dynamometer diagrams of reciprocating pumps....................... W 14

Dynamometers for testing windmills............................ 20

E.

Eagle Ford clay, water in, in Texas........................... W 276

Eagle Pass formation, water in, in Texas.................... A 18 II b

Eckel, E. C., Wells and springs in Mississippi..................... W 102

Eden shale, water in, in Kentucky ............................. W 233

water in, in Ohio.......................................... 259

"Edge water," related to oil................................ B 658

Edwards limestone, water in, in Texas.................. A 18 II b; GF 42,64

Egypt, ground water in, bibliography of.......................... 163

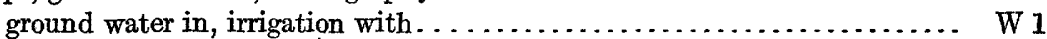

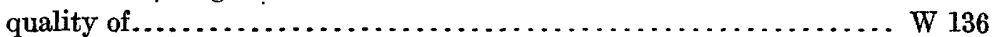

Eldridge, G. H., Geology of the Denver Basin, Colo.................... M 27

Electric currents in ground water.................................. W 258

Electrolytic bridge to determine total solids in water............. W, $137,138,139$ 
Elevators for lifting water. W 1,14

Ellensburg formation, water in, in Washington............ W 55, 118, 316; GF 86, 106

Ellis, A. J., Ground water in Paradise Valley, Ariz..................W 375 b

Ground water in the Hartford, Stamford, Salisbury, Willimantic, and

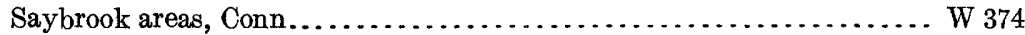

Ground water in the Waterbury area, Conn..................... W 397

Production of mineral water in $1916 \ldots \ldots \ldots \ldots \ldots \ldots \ldots \ldots \ldots \ldots . . . \ldots \ldots$ MR 1916

The divining rod, a history of water witching.................... W 416

Ellis, E. E., Water in crystalline rocks.......................... W 160

Water in crystalline rocks in Connecticut...................... W 232

Emmons, S. F., Geology of the Denver Basin, Colo ................... M 27

Emmons, W. H., Enrichment of ore deposits.......................... B 625

Enrichment of sulphide ores............................... B 529

Engines. See Power.

England, bibliography of ground water in....................... W 163

quality of ground water in................... B 330,491,616; MR 1916

Eocene formations. See under Tertiary; also see specific formations.

Equus beds, water in, in Kansas. . ............................. W 273

Escondido formation, water in, in Texas........................ W $375 \mathrm{~g}$

Europe, well drilling in . . . . . . . . . . . . . . . . . . . . . . . . . . . . W 257

See also specific countries.

Eutaw formation, water in, in Alabama.......................... S 2

water in, in Georgia.................................... W 341; S 4

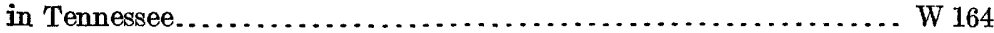

Evaporation, controlling factors in .............................. W 294

fluctuations of water table in relation to................ W $155,294,423$

from snow............................................. W 294

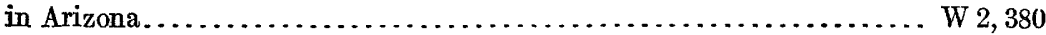

in California........................... $18,58,181,294,345 \mathrm{~h} ; \mathrm{S} 7$

in Colorado...................................... W $358 ;$ M 27

in Florida. . . . . . . . . . . . . . . . . . . . 319

in Georgia. . . . . . . . . . . . . . . 4

in High Plains........................................ $21 \mathrm{rv}$

in Kansas............................................ 153 ; B 140

in Nebraska............................................ B 140

in Nevada...................................... W $375 \mathrm{~d}, 423$

in New Mexico. . . ................................. W 275, 358

in Texas......................................... 358 ;F 166

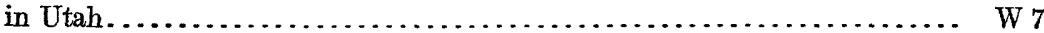

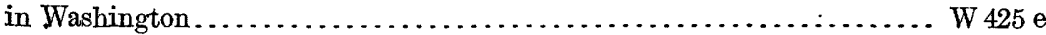

measurement of . . . . . . . . . . . . . . . . . . . . . . . . . . . . . . . . W 294

of ground water, experiments with............... W 153, 294; A 19 II b

in Arizona.......................................... 2,380

in California.............................. 294, $345 \mathrm{~h} ; \mathrm{S} 7$

in Florida. . . . . . . . . . . . . . . . . . . . 319

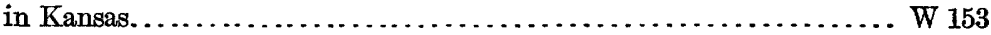

in Nevada....................................... $375 \mathrm{~d}, 423$

in New Mexico.............................................. 275

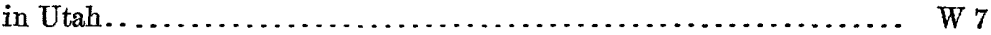

measurement of....................................... W 294

Explosives, methods of using, in wells.................... W 223, 255, 257

Exports of mineral waters. See under Mineral waters. 
F.

Fairbanks, H. W., San Luis folio, Cal.......................... 101

Fallacies regarding ground water. . . . . . . . . . . . . . . . . . . . . . . . 278

Farm supplies of ground water................................. W 255

Fault planes, water in. . ............................ W 114, 232; B 319

Faults, artesian water in relation to.................. W 6, 375 g; A 18 In b

ground water in relation to, in Arizona........................ W 380 in California................................ 142, 294, 338, $345 \mathrm{~h}$

in Connecticut........................................ W 232

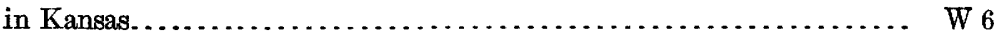

in New Jersey ............................................. 167

in Nevada....................................... 365,423

in New Mexico.......................................... 199

in Pennsylvania .................................... 167

in Texas.................................. W $375 \mathrm{~g} ; \mathrm{A} 18 \mathrm{II}$ b

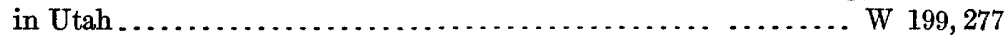

movements of ground water in relation to . . . . . . . . . . . . . . $345 \mathrm{~h}$

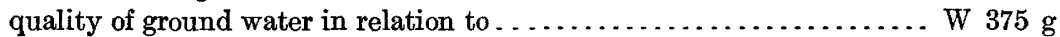

springs in relation to, in Arizona............................. W 380

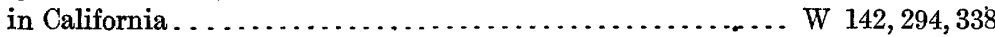

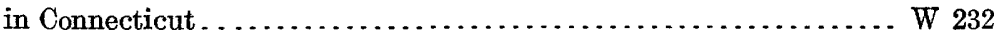

in Nevada...................................... 365,423

in New Mexico..................................... GF 199

in Texas......................................... 18 п b

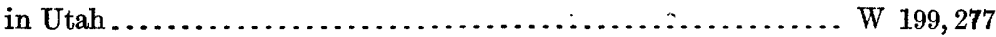

thermal springs in relation to.......... W 142, 227, 338, 365; A 14 II b; GF 199

water table in relation to........................... W 294, $345 \mathrm{~h}, 423$

Fayette sandstone, water in, in Texas.......................... $375 \mathrm{~g}$

Fenneman, N. M., Geology of the Boulder district, Colo ................ B 265

Geology and mineral resources of the St. Louis quadrangle, Mo.-Ill....... . B 438

Oil fields of the Texas-Louisiana Gulf Coastal Plain................ B 282

Field assays of water, methods of making...................... 151, 398

See also Analyses, Analysis, and Assays.

Figures. See Illustrations.

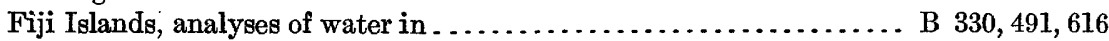

Filter galleries. See Infiltration ditches and tunnels.

Filtration of water. See Purification.

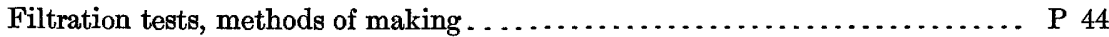

Finding water in deserts.............................. W 224,423; B 308

See also Watering places on routes of travel.

Finishing wells in sand.

W $256,293,343$ See also Well construction.

Finlay, G. I., Colorado Springs folio, Colo ...................... GF 203

Firing. wells, methods of . ........................... W 223, 255, 257

Fisher, C. A., Geology and underground waters of the Roswell artesian area,

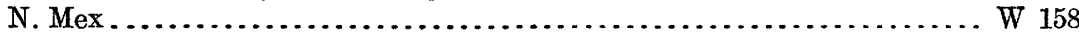

Geology and water resources of the Bighorn Basin, Wyo............ P 53

Geology and water resources of the Great Falls region, Mont ......... W 221

Nepesta folio, Colo...................................... GF 135

Fissility, ground water in relation to ....................... 223, 232

Fissures, movement of water through......................... A 19 II b springs in relation to...................... W 114; B 199; A 18 II b; S 9

Fleck, on flow of air through soils............................ 19 n b 
Flood control by feeding into underground reservoirs.

Flood-plain deposits. See Alluvium.

Flooding of oil wells.................................. 91,257

Floods in relation to ground water.............. $153,258,345 \mathrm{a}, 425 \mathrm{~b}$; $\mathrm{S} 6$

Florida, areas, all of State............................... W 114, 319

areas, Duval County..................................... B 298

Jacksonville........................................ B 298

St. Augustine . . . . . . . . . . . . . . . . . . . . . . . . 67

Sumter County . . . . . . . . . . . . . . . . . . . . . . . . . . . . B 298

Volusia County...................................... B 298

artesian water in . . . . . . . . . . . . . . . . . . . . . . . . 319

bibliography of ground water in ...................... W 114, 120, 163

large springs in................................. $319 ; \mathrm{A} 14 \mathrm{\text {Irb}}$

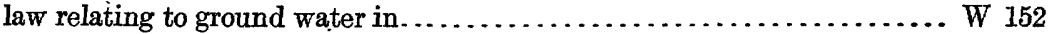

mineral water in........................ W 114; B 32; MR 1883-1916

public water supplies in . . . . . . . . .

quality of ground water in ..................... $102,319,364$; B 32

spring records for.................................. 27,$319 ;$ B 32

well records for............................. 57,$149 ;$ B 264, 298

Flow of air and water through porous media, methods of measuring..... A 19 In b

Flow of ground water. See Movements of ground water.

Flow of wells. See Wells and Artesian wells.

Flowage, zone of, ground in relation to.......................... M 47

Flowing wells. See Artesian water and Artesian wells.

Fluctuations of water table. See Water table.

Fluorescein, bibliography of ......................................... 160

for determining movements of ground water.................... 160

Fluorides in ground water.......................... B 47; M R 1911 n n

Flushing of wells. See Well construction.

Flynn, B. H., The Sandusky, Maumee, Muskingum, and Miami drainage

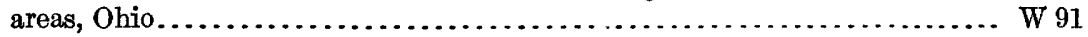

Flynn, Margaret S., The Sandusky, Maumee, Muskingum, and Miami drainage

areas, Ohio............................................... W 91

Foaming of water in boilers, quality in relation to........W 254, 256, 259, 274, $341,398,399 ;$ S 9

Foaming and priming constituents of water, methods of calculating....... W 274, 398

Foliation planes, water in . ................................ B 319

Forbes, R. H., Agriculture in San Simon Valley, Ariz.-N. Mex.......... W 425 a

Agriculture in Sulphur Springs Valley, Ariz ..................W 320

Force pumps................................................. 14

Forests, water table in relation to................................... 155

Fortier, Samuel, Seepage in northern Utah. .................... W 7

Fossil sea water. See Connate water.

Fossils in drillings from wells............................ W 293; P $90 \mathrm{~h}$

Fountain springs, construction of........................... 145

Fox Hills formation, water in, in Colorado............... W 9; M 27; P 32

water in, in Kansas...................................... P 32

in Nebraska................................. P 32

in North Dakota............................ B 575; GF 181

in South Dakota.......................... W 227; B 575; P 32

in Wyoming...................................... P $32 ;$ GF 173

Fracture, zone of, ground water in relation to ..................... M 47

Fracture zones, water in................................... GF 149 
France, bibliography of ground water in W 163

quality of mineral water in

radioactive waters in

MR 1913 II h

springs in, yield of

A 14 II b

Franconia sandstone, water in, in Minnesota......................... GF 201

Fredericksburg group, water in, in Texas ....................... 21 vIr

Freezing of wells.

$\mathrm{W} 29,256,258$

Friction of ground water..................................... 47

Frinkle, F. C., Pumping underground water in southern California........ W 146

Frontier formation, water in, in Wyoming . . . . . . . . . . . 656

Frost, confining beds for artesian water produced by.................. B 319

effect of, on ground-water supply........................... 145

Fry-pan deposits, water in, in Texas.......................... A 18 II b

Fuller, M. L., A ground-water problem in southeastern Michigan......... W 145

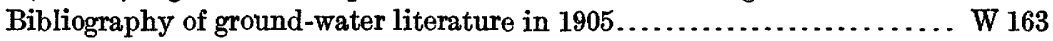

Bibliography of United States Geological Survey papers on underground

water............................................... 120

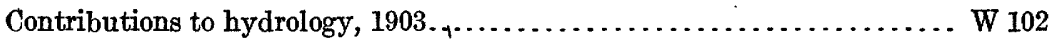

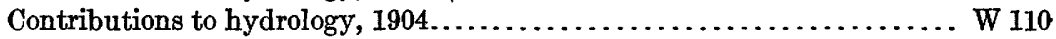

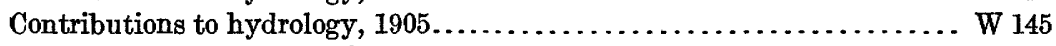

Controlling factors of artesian flows . . . . . . . . . . . . . . . . B 319

Ditney folio, Ind.......................................... 84

Drainage by wells....................................... 258

Flowing wells and municipal water supplies in the southern peninsula of

Michigan ........................................... W 182, 183

Freezing of wells and related phenomena....................... W 258

Gaines folio, Pa.-N. Y.................................. 92

Geology and underground waters of southern Minnesota...........W 256

Improvement of water in wells.......................... W 160

Large springs of Ozark region, Mo.-Ark. . . . . . . . . . . . . . W 145

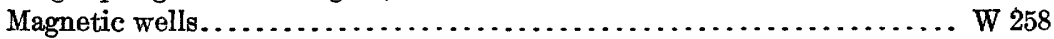

Patoka folio, Ind.-Ill.................................... 105

Peculiar mineral waters from crystalline rocks....................W 160

Production of mineral waters. . . . . . . . . . . . . . . . . . . . . . . MR 1905

Protection of shallow wells in sandy. deposits................... W 258

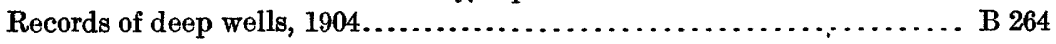

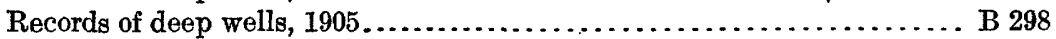

Representation of wells and springs on maps:..................... W 160

Significance of the term "artesian" .......................... W 160

Total amount of free water in the earth's crust.................... W 160

Triassic rocks of the Connecticut Valley....................... W 110

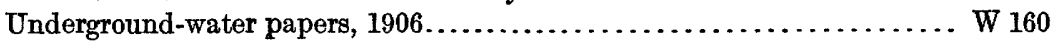

Underground waters for farm use............................. W 255

Underground waters of District of Columbia.................... W 114

Underground waters of eastern United States................... W 114

Underground waters of Florida............................ W 114

Underground waters of Maryland . . . . . . . . . . . . . . . . . . . . W 114

Underground waters of New Hampshire.................... W 114

Underground waters of North Carolina....................... W 114

Underground waters of Pennsylvania......................... W 114

Underground waters of southwestern Ohio.................... W 259

Underground waters of Virginia................................ W 114

Underground waters of West Virginia........................ W 114

Uses of underground waters of southern Louisiana..................W 101 
G.

Gagen, on flow of water through soils.

A $19 \mathrm{mb}$

Gages for determining vacuum in purmps.

Gages for recording water levels in wells.

Gale, H. S., Potash in Death Valley, Cal

Potash tests at Columbus Marsh, Nev.

B $540 \mathrm{n}$

Water supply of Rangely oil district, Colo...................... B 350

Water resources of the Cowee and Pisgah quadrangles, N. C........... W 110

Galena limestone, water in, in Illinois................. W 114; B 506; GF 81, 200

water in, in Iowa.............................. W 293; GF 145, 200

in Minnesota............................................... 256

in Wisconsin................................... W 114; GF 145

Galleries. See Infiltration ditches and tunnels.

Gas in relation to ground water.

B 658

Gases, artesian flows influenced by

A 5 c

Gases in ground water.

M $47 ;$ B 395

methods of testing for

B 395

Gasoline engines. See under Power.

Géologic causes of fluctuations of ground-water levels.

Geologic work of ground water.

W 155

Georgia, areas, all of State

M 47; B 529, 606

$\mathrm{W} 102,114 ; \mathrm{S} 4$

areas, Chatham County...................................... B 298

Coastal Plain. . . . . . . . . . . . . . . . . . . . . . . . 138

- Decatur County......................................... B 298

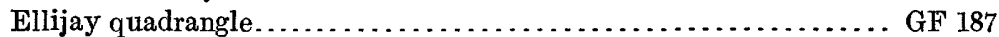

Fulton County . . . . . . . . . . . . . . . . . . . . . . . . . . . . B 298

Pierce County. . . . . . . . . . . . . . . . . . . . . . . . . . . . . . . B 298

Quitman and vicinity................................. 110

Savannah and vicinity....................... W 67; B 298; S 11

Tattnall County . . . . . . . . . . . . . . . . . . . . . . . . . . . B 298

artesian water in........................... W 67, 341; B 138; S 4, 11

artesian water power in .................................. S 4

bibliography of ground water in ............. W 57, 114, 120, 149, 163; S 4

blowing springs in . . . . . . . . . . . . . . . . $4 \ldots \ldots \ldots \ldots \ldots \ldots \ldots$ S

blowing wells in ......................................... 258 ; 4

drainage into wells in . . . . . . . . . . . . . . . . . . . . . . 160

irrigation with ground water in.................................. S 4

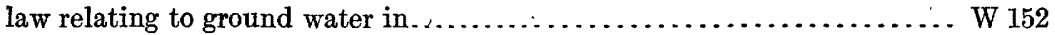

mineral water in................ W 114, 160; B 32; MR 1883-1916; GF 187

power from artesian wells in .............................. S 4

public water supplies in...................W 67, 102, 341; B 138; S 4, 11

quality of ground water in . .............W 102, 160, 341; B 32, 138; S 4, 11

spring records for..................... W 36, 127, 168, 197; B 32; S 4

waters, peculiar, in crystalline rocks of....................... W 160

well records for........................... W 57, 149; B 264, 298; S 4

Gering formation, water in, in Nebraska................. A 19 Iv c; P 17

Germany, bibliography of ground water in....................... W 163

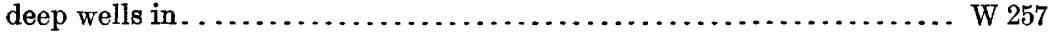

mineral waters in ............................... B 606; MR 1916

quality of ground water in .............. W 330,491, 529,606, 616; MR 1916

radioactive water in ..................................... $1913 \mathrm{~m} \mathbf{h}$

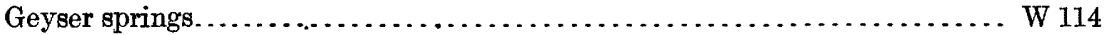

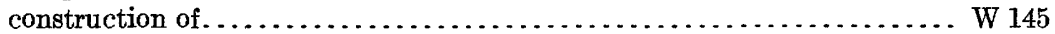

in Nevada.............................................. 365 
Geysers, analyses of water from............. W 364; B 47, 330, 491, 616; A 9 d

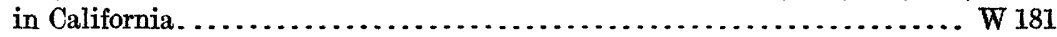
in Yellowstone National Park............... W 364; A 9 d, 14 II b; B 47, 395

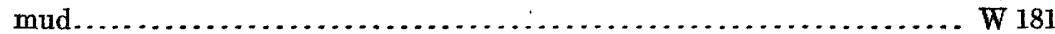
radioactivity of watier from.................... B 395; MR 1913 II $h$ water yielded by . . . ............................... 14 II b Gila conglomerate, water in, in Arizona............... W 320, 425 a; GF 111 Gilbert, G. K., Pueblo folio, Colo.............................. GF 36 Underground water of Arkansas Valley, Colo.................... A 17 II f

Giles, J. M., stream measurements for $1905 \ldots \ldots \ldots \ldots \ldots \ldots \ldots \ldots \ldots \ldots$ W 173

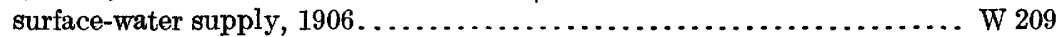

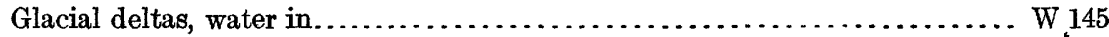

Glacial drift, drainage into wells in ............................. W 258 water in, in Arkansas.............................. W 145; GF 140 in Connecticut............................ 114, 232, 374, 397 in eastern United States. .............................. W 114 in Illinois......... W 114; A $17 \mathrm{II} \mathrm{h;} \mathrm{M} \mathrm{38;} \mathrm{GF} \mathrm{67,} \mathrm{105,} \mathrm{185,} \mathrm{188,} \mathrm{195;} \mathrm{S} 3$ in Indiana............... W 21, 26, 113, 114, 254; A 18.rv b; GF 67, 105 in Iowa. . . . . . . . . . . . . . . . . . . . . . . . . . . . . . . W 293 in Kansas........................................ 273 GF 206 in Maine................................. 145, 223; GF 149

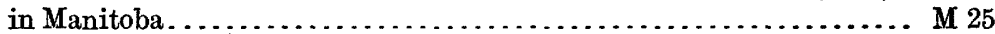
in Massachusetts......................................... 145 in Michigan....................... 3 30, 31, 182, 183; GF 155, 206 in Minnesota......................... W 256; M 25; GF 117, $201^{\bullet}$ in Missouri.................................. 195; GF 206 in Montana...................................... 221, $400 \mathrm{~b}$ in Nebraska .................................. 12; GF 156 in New Jersey.................................. 157,161 in New York.................. W 110, 114, 145; GF 157, 169, 190 in North Dakota. ..................... A 17 II g; M 25; GF 117, 168 in Ohio..................... W 114, 259; A $18 \mathrm{IV}$ b, $19 \mathrm{IV}$ b; GF 197 in Pennsylvania......................................W 114 in South Dakota.................... W 34, 90, 227; A 17 пा g; GF 96, $97,99,100,113,114,156,165$

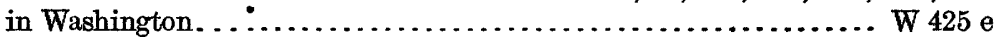

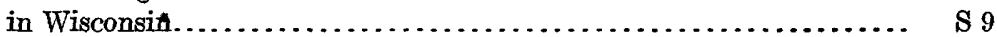
in preglacial channels, water in.................. W 145, 293; A 19 rv b See also Glacial outwash and Till.

Glacial outwash, water in, in Connecticut................ W 232, 374, 397

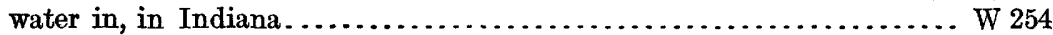

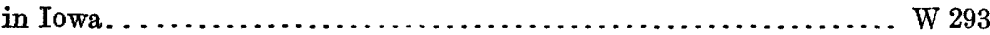

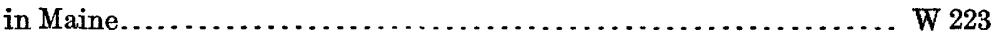

in Michigan . . . . . . . . . . . . . . . . . . 183

in Minnesota......................................... W 256

in Ohio.............................................. W 259

in Washington. ........................................... $425 \mathrm{e}$

See also Glacial drift.

Glen Rose formation, water in, in Texas................... A 21 vII; GF 64

Glenn, L. C., Underground waters of South Carolina...................W 114

Underground waters of Tennessee and Kentucky west of Tennessee River

and of an adjacent area in Illinois.............................. W 164

Water resources of Kentucky and Tennessee................. W 102, 114

Gneiss, water in......... W 106, 114, 160, 223, 232, 255, 257; GF 147, 157, 162, 191

See also Crystalline rocks. 
Gold in ground water. P 94; B 529, 625

Gooch, F. A., Analyses of waters of Yellowstone National Park.......... B 47

Goodell, E. B., Laws forbidding pollution of inland waters............... W 152

Gordon, C. H., Geology and underground waters of northeastern Texas........ W 276

Geology and underground waters of the Wichita region, north-central Texas. W 317

Gould, C. N., Geology and water resources of Oklahoma............... W 148

Geology and water resources of the eastern portion of the Panhandle of

Texas................................................ W 154

Geology and water resources of the western portion of the Panhandle of

Texas................................................. W 191

Grand Falls chert, water in, in Kansas............................. GF 148

.water in, in Missouri....................................... GF 148

Grand Gulf formation, water in, in Alabama....................... S 2

water in, in Mississippi.................................... 159

Graneros shale. See Benton formation.

Granite, water in................. W 114, 160, 223, 232, 255, 257, 258, 277, $345 \mathrm{~g}$,

See also Crystalline rocks.

374, 397; A 18 II b; GF 45, 147, 149, 151, 158

Grant, U. S., Lancaster-Mineral Point folio, Wis.-Iowa-Ill. . .............. GF 145

Water resources of the Mineral Point quadrangle, Wis............... W 145

Gravel, drainage into wells in ................................. 258

flow of water in, experiments with........ W 112, 136, 140, 141, 153, 184, 258; P 44

in buried channels, water in ..................... W 273, $293 ; \mathrm{A} 19 \mathrm{rv} \mathrm{b}$

interbedded with lava, water in ........................ W 4; B 199

- $\quad$ porosity of ..........................................W 104, 136, 140 spreading flood water over................................ S 6 walls of wells formed of ............ W 256,293, 343, 345 a, 345 d, 345 g, $375 \mathrm{a}$ water in.................... W 6, 112, 114, 136, 153, 184, 223, 233, 255, $257,258,273,319,374,397,399,425$ b; $\mathrm{P} 44$

See also Alluvium, Glacial drift, Porosity, and Valley fill.

Gravel screens in wells ............... W 256, 293, 343, $345 \mathrm{a}, 345 \mathrm{~d}, 345 \mathrm{~g}, 375 \mathrm{a}$

Graydon sandstone, water in, in Missouri.........................W 195

Great Plains. See High Plains and specific States.

Greece, ancient, mineral water used in...................... 14 In b.

Greene, F. C., Leavenworth-Smithville folio, Mo.-Kans............... GF 206

Greenhorn limestone, water in, in Nebraska....................... GF 156

water in, in South Dakota............................ GF 156

See also Benton formation.

Gregory, H. E., Ground water in Hartford, Stanford, Salisbury, Willimantic, and Saybrook areas, Conn .............................. W 374

The Navajo country - a geographic and hydrographic reconnaissance.... W 380

Underground water resources of Connecticut..................... W 232

Underground waters of Connecticut.................... W 102, 114

Gregory, W. M., Flowing wells and municipal water supplies in the southern

peninsula of Michigan........................................ 183

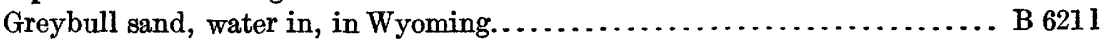

Ground-water level. See Water table.

Grover, N. C., Contributions to hydrology, $1914 \ldots \ldots \ldots \ldots \ldots \ldots \ldots \ldots \ldots \ldots \ldots$ W 345

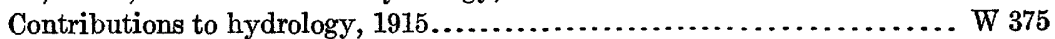

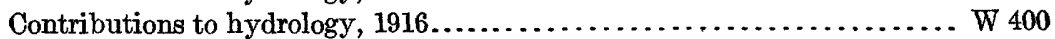

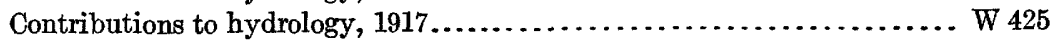

Grunsky, C. E., Irrigation near Bakersfield, Cal..................... W 17

Irrigation near Fresno, Cal................................. 18

Guides to watering places. See Watering places. 
Gypsum deposits, springs in.

H.

Hale formation, water in, in Arkansas.......................... GF 154 water in, in Oklahoma..................................... 154

Hall, C. M., Alexandria folio, S. Dak................................ 100

Casselton-Fargo folio, N. Dak.-Minn......................... GF 117

De Smet folio, S. Dak................................... GF 11

Geology and water resources of James River valley, S. Dak........... W 90

Hall, C. W., Geology and underground waters of Southern Minnesota . . . . ... W 256 Underground waters of Minnesota.......................... W 102, 114

Hamilton shale, water in, in Wisconsin............................ $\mathbf{S . 9}$

Hamlin, Homer, Water resources of the Salinas Valley, Cal............. W 89

Underflow tests along Los Angeles River......................... W 112

Hammond, G. A., Diamond-drill methods.......................... W 146

Hance, J. H., Potash in western saline deposits...................... B 540 p

Hanna, F. W., Stream measurements for $1905 \ldots \ldots \ldots \ldots \ldots \ldots \ldots \ldots \ldots$ W 170

Hannibal formation, water in, in Missouri........................... W 195

Hardness of ground water......... W 232, 254, 259, 273, 320, 338, 341, 343, 398, 399

Hare, R. F., Geology and water resources of Tularosa Basin, N. Mex......... W 343

Hares, C. J., Anticlines in Wyoming ........................... W $641 \mathrm{i}$

Lignite field of northwestern S. Dak........................ B 627

Harris, G. D., Underground waters of southern Louisiana................ W 101

Hatchetigbee formation, water in, in Alabama...................... S 2

Hawaii, areas, Hawaii Island ............................ 318, 336

areas, Kauai Island .................................. 318, 336

Maui Island............................ W 318, 336, 373, 445

Molokai Island ....................................... W 77

Oahu Island....................... W 318, 336, 373, 430, 445; S 12

artesian water in .......................... W 318, 336, 373, 430; S 12

bibliography of ground water in................................ 163

infiltration tunnels in .................................... 318,445

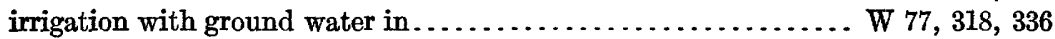

quality of ground water in................................. 77

quantity of ground water in................................ 77 ; 12

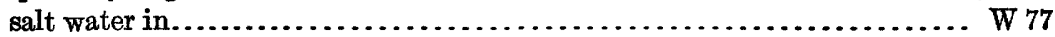

spring records for............................ W 318, 336, 373, 430, 445

wells in, yield of ........................... W 77, 318, 336, 373; $\mathrm{S} 12$

Hawkins, R., Water supply of Livingston County, Mo................ W 102

Haworth, Erasmus, Economic geology of Iola quadrangle; Kans.......... B 238

Underground waters of southwestern Kansas.................... W 6

Hawthorn formation, water in, in Florida.......................... W 319

Hay, Robert, Water resources of a portion of the Great Plains........... A 16 II $f$

Well records in Nebraska, Colorado, and Kansas..................... B 131

Head of ground water. See Artesian water and Water table.

Heald, K. C., Geologic structure of northwestern Pawhuska quadrangle, Okla. B 619 c Oil and gas geology of Foraker quadrangle, Okla................ B $641 \mathrm{~b}$

Hendrixson, W. S., Underground water resources of Iowa................ W 293

Henrietta limestone, water in, in Missouri............................ W 195

Hewett, D. F., Anticlines in southern part of Big Horn Basin............. B 656

Hicks, W. B., Composition of muds from Columbus Marsh, Nev.......... P 95 a 
High Plains, ground water on....................... A 21 rv c, 22 Iv c See also under individual States.

Highbridge limestone, water in, in Kentucky..................... W 233

Hill, J. M., Economic geology of Gilpin County and adjacent parts of Clear

Creek County, Colo.................................... P 94

Mineral deposits of Santa Rita and Patagonia mountains, Ariz........ B 575

Hill, R. T., A ustin folio, Tex................................ GF 76

Geography and geology of Black and Grand prairies, Tex.......... A 21 vII

Geology of the Edwards Plateau and Rio Grande Plain, Tex........ A $18 \mathrm{mb}$

Nueces folio, Tex............................................ 42

Hills, R. C., Elmoro folio, Colo............................. GF 58

Spanish Peaks folio, Colo...................................... 71

Walsenburg folio, Colo....................................... 68

Hinderlider, M. C., Stream measurements for $1904 \ldots \ldots \ldots \ldots \ldots \ldots \ldots \ldots$. . . . . . 131

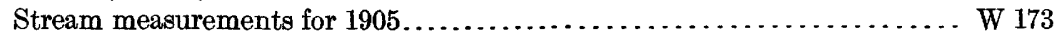

Hinds, Henry, Leavenworth-Smithville folio, Mo.-Kans................. GF 206

History of ground-water investigations, U. S. Geological Survey, prior to

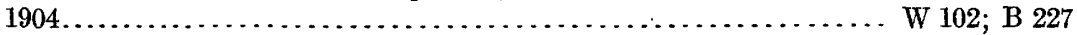

Holland, drainage with windmills in ............................... 20

ground water in, bibliography of ............................... 163

Hollick, Arthur, New York City folio......................... GF 83

Hollister, G. B., Water of a gravel-filled valley near Tully, N. Y .......... W W 145

Hood, O. P., Tests of pumps and water lifts........................... W 14

Horsepowers for lifting ground water ............................... 14

Horton, R. E., Drainage into wells............................... W 145

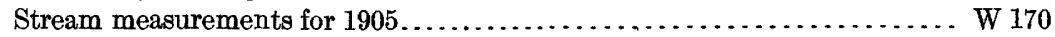

Surface streams of Long Island, N. Y ..................... P 44

Weir experiments, coefficients, and formulas................. 150, 200

Hot Springs. See Springs and Temperature.

Hot ground waters. See under Temperature.

Howe, Ernest, Ouray folio, Colo . . . . . . . . . . . . . . . . . . . . . . . GF 153

Silverton folio, Col...................................... 120

Hoyt, J. C., Stream measurements for $1903 \ldots \ldots \ldots \ldots \ldots \ldots \ldots \ldots \ldots \ldots \ldots$. . . . . . . . . 99

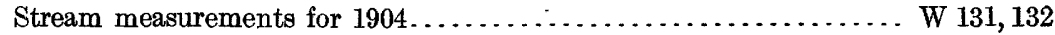

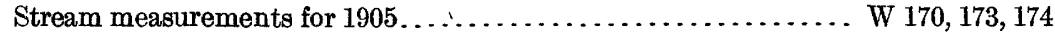

Hubbard, G. D., Columbus folio, Ohio. ....................... GF 197

Hudson beds, water in, in Indiana................................ W 114

Hudson shale, water in, in Michigan.......................... W 114

Hueco limestone, water in, in Texas ............................ GF 195

Huronian quartzite, water in, in Wisconsin..................... S 9

Hutson, W. F., Irrigation systems in Texas........................ W 13

Hydration produced by ground water............................ M 47

"Hydraulic grade plane"................................. A 21 IV c

Hydraulic rams for lifting water..................... 1, 254, 256, 258; S 8

Hydrographic contours. See Maps showing contours of water table.

Hydrology, division of, early work of ...................... W 102; B 227

Hydrology. See specific areas.

Hydrolysis of ground water.................................... 47

Hygrometric water.......................................... 47

I.

Ice for domestic water supplies.............................. B 350

Ice manufacturing, quality of water for......................... W 233

Iceland, bibliography of ground water in ......................... W 120

quality of ground water in.......................... B 330,491, 616 


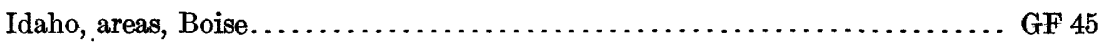

areas, Curlew Valley................................... W 333

Nampa quadrangle.................................. GE 103

Nez Perce County.................................. W 53,54

- Pocatello Valley ..................................... W 333

Silver City quadrangle............................ GF 104

Snake River Plains................................... B 199

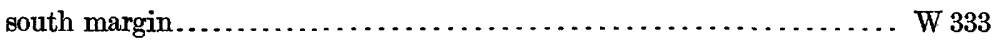

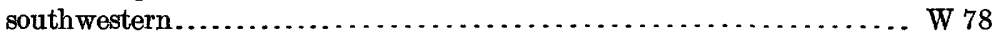

artesian water in..................... W 54, 78; B 199; GF 45, 103, 104

bibliography of ground water in....................... W 120, 163

early irrigation with ground water in....................... 16 II e

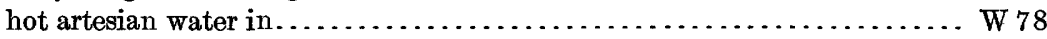

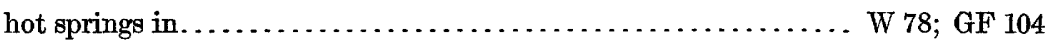

irrigation with water from............................ 104

infiltration tunnels in.................................... 54

irrigation with ground water in................. A 16 II e; GF 103, 104

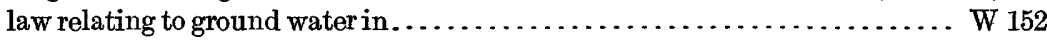

mineral waters in........................... B 32; MR 1883-1916

quality of ground water in......................... $32 ;$ GF 45

spring records for........................... $85,135,393 ;$ B 32

temperature of ground water in................W 78; B 32, 199; GF 104

well construction in . . . . . . . . . . . . . . . . . . . . . . . . 78

well records for.............................. W 57, 149; B 264, 298

Igneous contacts, water in ................................... B 319

Igneous rocks, sodium carbonate water in relation to................. W 320 See also Crystalline rocks and Lava.

Illinois, areas, all of State.................... W 114; A 17 II h; M 38

areas, Belleville quadrangle................................ GF 195

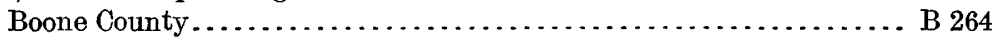

Breese quadrangle. . . . . . . . . . . . . . . . . . . . . . . . . . 195

Brown County. . . . . . . . . . . . . . . . . . . . . . 298

Chicago quadrangle................................... GF 81

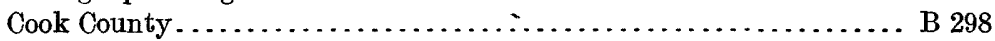

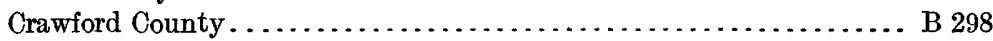

Danville quadrangle. . . . . . . . . . . . . . . . . . . . . . 67

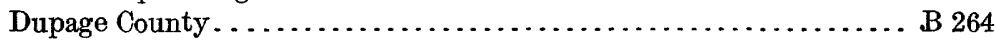

East St. Louis district. . . . . . . . . . . . . . 3

Galena quadrangle................................. GF 200

Hancock County ........................................... B 298

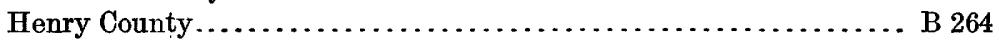

Herrin quadrangle...................................... 185

Lancaster quadrangle................................ GF 145

La Salle County ................................... B 264, 298

Mineral Point quadrangle. . . . . . . . . . . . . . . . . . . . GF 145

Murphysboro quadrangle ............................ GF 185

Patok quadrangle.................................. GF 105

Peoria quadrangle. . . . . . . . . . . . . . . . . . . . . . . . . B 506

Rockford . . . . . . . . . . . . . . . 67

St. Louis quadrangle ................................. B 438

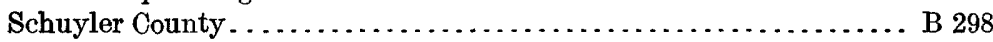

southern..................................................... 164

Springfield quadrangle.................................. GF 188

Tallula quadrangle.................................. GF 188 
Illinois, artesian water in...........W 67; B 438, 506; M 38; GF 67, 81, 195; S 3 bibliography of ground water in ..............W 57, 114, 120,149, 163, 164 law relating to ground water in .............................. W 152 mineral waters in . ....................... W 114; B 32; MR 1883-1916 public water supplies in............... A $17 \mathrm{~m} \mathrm{~h}$.M 38; B 506; GF 195; 33

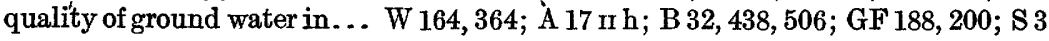

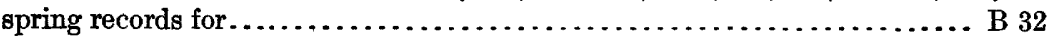

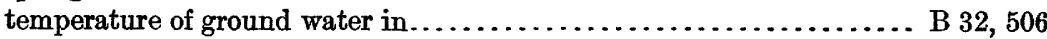

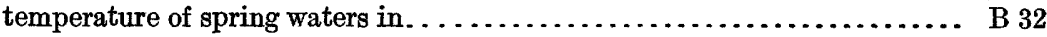
well records for............................. W 57, 149; B 264, 298 wells in, construction of . . . . . . . . . . . . . . . . . . . . . 164

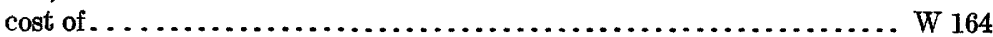

Illustrations showing apparatus for determining color in water............ W 151 apparatus for determining thorium in ground water................ B 395 apparatus for making underflow measurements............ W 110, 140; P 44 area having ground-water discharge......................... 294 artesian reservoir, section of . . . . . . . . . . . . . . . 5 artesian springs............................... 34,$90 ; \mathrm{A} 21$ IV $\mathrm{c}$

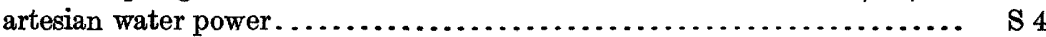
augers for boring wells................................... W 257 bedding planes in limestone with water oozing therefrom .......... W 254 blowing spring.............................................. 4

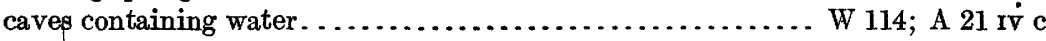
dams, subsurface........................................ 67 deposits made by hot springs......................... W $338 ;$ P 53

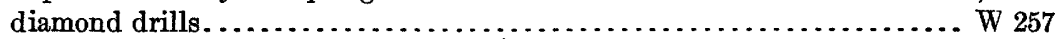

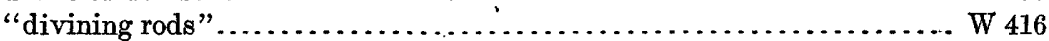
drainage wells.............................................. 145 drilling rigs, California .............................. 110, 140

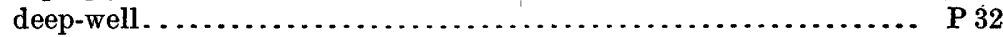

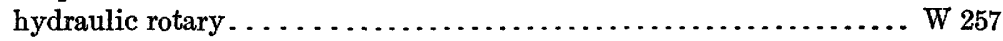
jetting process................................ $30,140,257$ oil-well . . . . . . . . . . . . . . . . . . . . . . . . . 113

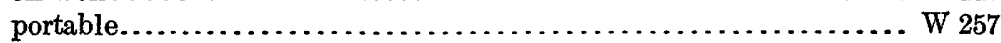

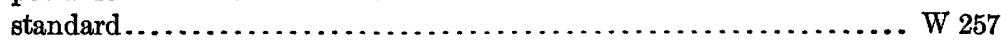

used in'Arkansas. . . . . . . . . . . . . . .

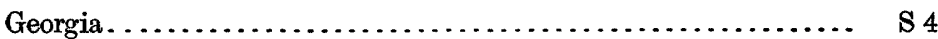

Great Plains. . . . . . . . . . . . .

Indiana. . . . . . . . . . . . . . . . . . . . . . . . . . 254

Louisiana . . . . . . . . . . . . . . . 46

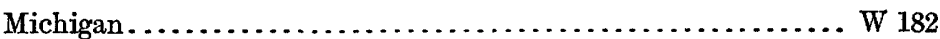

South Dakota ................................... A 18 IV c

Texas................................................... 141

wash-process.............................................. 145

drilling tools. ............................................. W 257

drive point and screen .................................. 257

driving outfit for tubular wells............................ W 159

dynamometers............................................... W 20

evaporation pans........................................ W 294

fault springs.................................................... 199

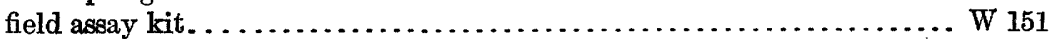

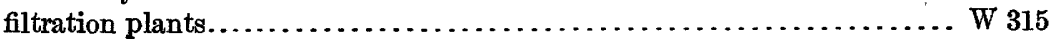

fissure springs. $\ldots \ldots \ldots \ldots \ldots \ldots \ldots \ldots \ldots \ldots \ldots \ldots \ldots \ldots \ldots$, A 21 .VII 
Illustrations showing flowing wells.

W 67, 114; A 22 IV c

in Alabama

in Arizona

A. 21 IV a

in California

W 140, 294

in Colorado

W 240; A 21 IV a; P 32, 52

in Florida W 319

in Georgia W 341; S 4

in Indiana W 254

in Kansas W 6; A 21 IV c

in Kentucky W 164

in Michigan W 145,182

in Nebraska. $\mathrm{W} 29,215,216$

in Nevada. ... W 365

in New Mexico ...................................... W 158, 343

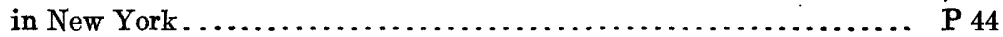

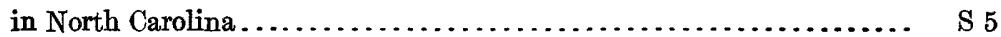

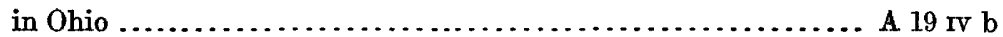

in South Dakota.................... W 90; A 17 II g; $21 \mathrm{rv} \mathrm{b;} \mathrm{P} 32$

in Tennessee.................................... 164

in Texas .............................. 13, 190; A 18 II b; B 164

in Washington ............................................ 55

geysers in Yellowstone National Park . . . . . . . . . . . . . . . . . . . . . B 396

infiltration ditches in California $\ldots \ldots \ldots \ldots \ldots \ldots \ldots \ldots \ldots \ldots \ldots$ A 21 Iv a

interference of wells . . . . . . . . . . . . . . . . . . . . . . . . . . . . . . W 254

Jackson candle turbidimeter . . . . . . . . . . . . . . . . . . . . . . . . W 151

lakes produced by artesian water ......................... 18 Iv c

lifting water, curious devices for............................ W 1

mud volcanoes in Califormia .................................. W 225

perforators for well casings ............................. 110, 140

pumping plants in Arizona............................. 135, 320

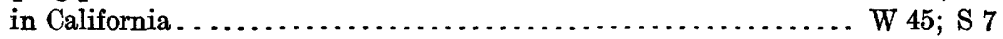

in New Mexico............................................ 158

portable............................................... 45

pumps and other lifting devices, curious and ancient................ W 1

used in Egypt..................................... W 1

used in India...................................... W 1

reservoirs for well water....................... W 10, 13, 20, 154; B 319

sink holes................... W 67, 114, 343; A 21 rv b, 21 rv c; P 53,65

in Great Plains . . . . . . . . . . . . . . . . . .

in New Mexico....................................... W 343

in Wyoming ................................. $21 \mathrm{rv}$ b; $\mathrm{P} 53,65$

siphon elevators................................................ 1

soil evaporation tanks........................................ 294

solution channel on fault line............................... B 319

solution channels in limestone....................... W 254, 259, 374

spring mounds in New Mexico . . . . . . . . . . . . . . . . . . . . . . . W 343

springs, artesian .......................... 34,$90 ; A 21$ rv c

at outcrop of impervious floor ......................... W 67

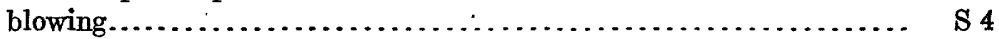

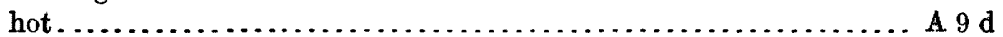

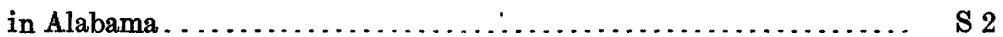

in Alaska . . . . . . . . . . . . . . . . 418

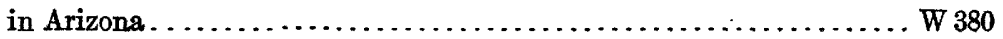


Mlustrations showing springs in California. . . . . . . . . . . . . . . . . . W 338 springs in Georgia...................................... W 341 S 4 in limestone.................................. W 317; A 21 iv b in Michigan............................................. W 182 in Missouri ............................................ W 195

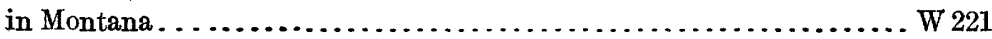

in Nebraska.......................................... 12

in New Mexico . ................................... 158,343 in Texas.................................. A 18 I b; A 21 vir in Yellowstone National Park.......................... A $9 \mathrm{~d}$ of large size.............................. W. 221, 341; A 18 m b produced by faults . . . . . . . . . . . . . . . . . . . . . . . . . . W 199 produced by fissures................................. A 21 vn thermal .......................................... A $9 \mathrm{~d}$

stalactites deposited by ground water in Texas................ A 18 In b stock-watering places.................................. W 154, 191 stovepipe casings ............................................ 140

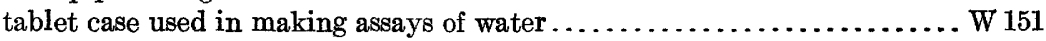
tank filled by artesian pressure............................ P 32 "tanks" for watering live stock...................... W 154; A 22 Iv c terraces formed by hot springs............................ A $9 \mathrm{~d}$ travertine deposited by hot springs in California . . . . . . . . . . . . W 338 deposited by springs in New Mexico........................ W 343

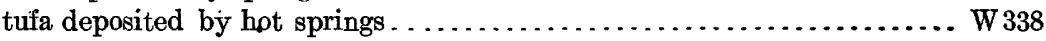
deposited by ground water in Texas.................... A 18 In b underflow measurements . .....................W 110, 140; P 44; S 4 underground streams....................W 67, 114,255; A 21 rv c; S 4 wash drilling ................................................... 145 water holes in Colorado.................................... P 52

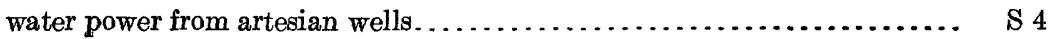
water wheels for lifting water........................ $1,4,13,319$

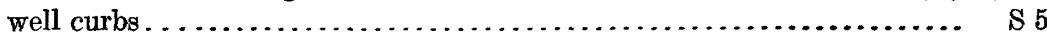
well derricks . . . . . . . . . .

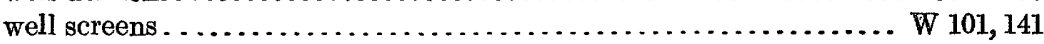
well sweeps.................................................... S 5 well, natural...................................... 6 ; 21 rv c wells, combination dug and drilled...................... A 22 rv c in the desert. . . . . . . . . . . . . . . . . . . . . . . . . . . . . 224

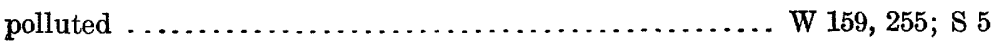
windlass and well curb................................ W 29 windmills................ W 1, 6, 8, 20,29, 41, 42, 154, 191; A 21 rv c, 22 rv c curious.................................. $20,29,41,42$ Dutch................................................. 41 for watering live stock . . . . . . . . . . . . . W 191; A $21 \mathrm{rv} \mathrm{c,} 22 \mathrm{rv} \mathrm{c}$ homemade............................................ W 1 See also Maps.

Imbibition .

See also Absorption.

Imports of mineral waters. See under Mineral waters.

Imprisoned sea water. See Connate water.

Improvement of water in wells............................. W 160

Inclusions of water in rocks................................... M 47

Incrustations on well screens, analyses of ................... W 256,293

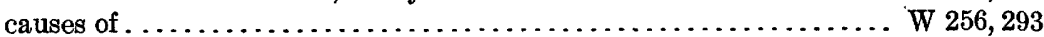

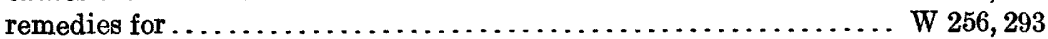


Indexes. See Bibliography.

India, bibliography of ground water in

W 120,163

irrigation with ground water in ....................... W $1 ;$ A 11 II $\mathrm{c}$

pumping in . . . . . . . . . . . . 1,14

pumps and other water lifts used in ....................... W 1,14

Indian Territory. See Oklahoma.

Indiana, areas, all of State

W 114; A 18 iv b

Danville quadrangle . . . . . . . . . . . . . . . . . . . . . . . FF 67

Delaware County ................................... B 298

Ditney quadrangle..................................... GF 84

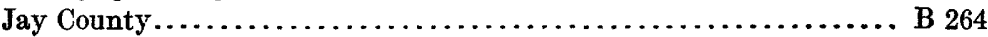

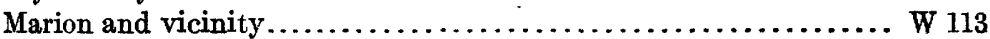

Martin County....................................... B 298

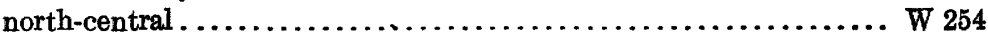

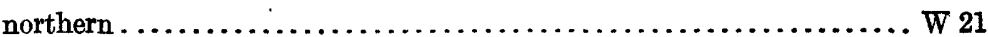

Patoka quadrangle.................................... GF 105

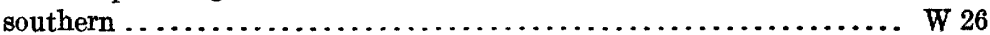

Vanderburg County.............................. B 298

artesian water in .................. W 21,26, 114, 254; A 18 Iv b; GF 67

bibliography of ground water in...............W 57,114, 120, 149, 163

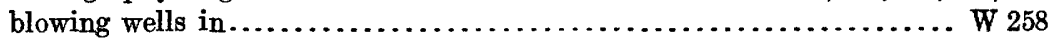

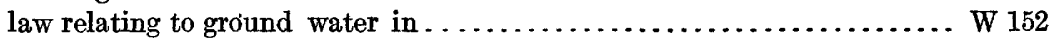
mineral waters in............ W 114; A 18 Iv b; B 32; M R 1883-1916; GF 84 public water supplies in ....................... W 254; A 18 iv b quality of ground water in............... W 254; A $18 \mathrm{iv} \mathrm{b;} \mathrm{B'32;} \mathrm{GF} 84$

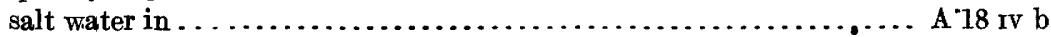

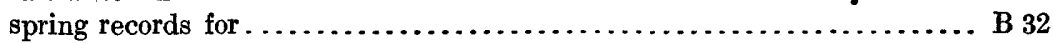

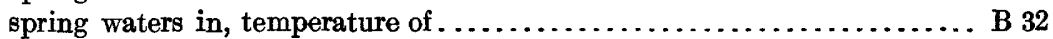

well construction in .................................... W 254

well records for ............................. W 57, 149; B 264, 298

Indications of ground water....................... W 224, 423; B 308

Industrial uses of ground water. See under Quality.

Industrial wastes, drainage of, into wells....................... W 258

Infiltration ditches and tunnels, construction of . . . . . . . . . . . . W 67, 116, 380

cost of ........................................ 116, 184

in Arizona...................................... 104, 380

in California........................ W 116; A $19 \mathrm{Ir} \mathrm{b,} 21$ Iv a; GF 193

in Colorado............................................ W 240

in Connecticut........................................ 374,397

in Hawaii. . . . . . . . . . . . . . . . . . . . . . . . . . . . . . . . . W 445

in Idaho . . . . . . . . . . . . . . . . . . . 54

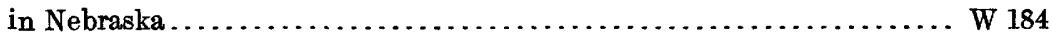

in New Mexico................................... 188, 275, 343, 380

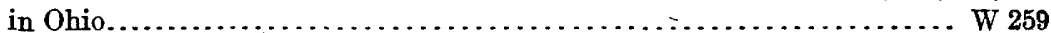

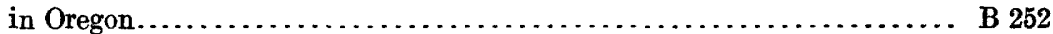

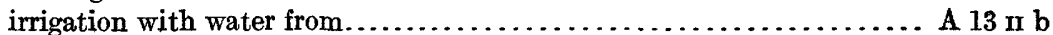

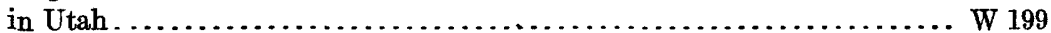

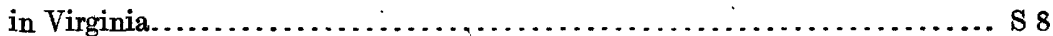

Instruments. See Apparatus.

Intake of ground water. See Absorption of water, Origin of ground water, and

Quantity of ground water.

Interference of wells....... W 67, 122, 137, 184, 223; A 19 II b; M 27; GF 97; S 8, 9, 12

Interference with springs and streams, law relating to................W 122

Iodine in ground water. 
Ione formation, water in, in California. W 375 a; GF 138

Iowa, areas, all of State ................................. 114, 293

areas, Blackhawk County.................................... B 298

Cherokee County....................................... B 264

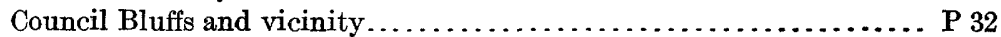

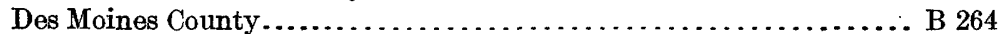

Elk Point quadrangle....................................... GF 156

Floyd County .......................................... B 298

Galena quadrangle.................................... GF 200 .

Lancaster quadrangle ..................................... GF 145

Louisa County . . . . . . . . . . . . . . . . . . . . . . . . . . . . . . . B 298

Mahaska County ........................................ 298

northwest corner. ....................................... 215

Scott County ....................................... B 264, 298

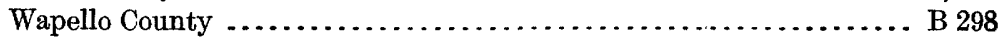

Waterloo............................................. W 145

artesian water in ........................ W 145, 293; P 32; GF 145, 156

bibliography of ground water in .................. W 57,114,120,149, 163

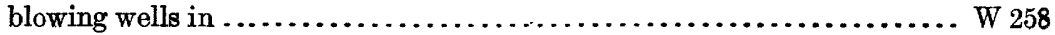

law relating to ground water in................................ 152

mineral waters in ........................ W 293; B 32; MR 1883-1916

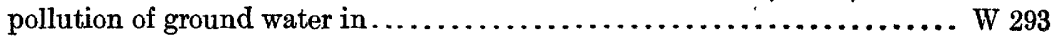

public water supplies in ................................ W 293

quality of ground water in................W 293, 364; B 32; GF 145, 200

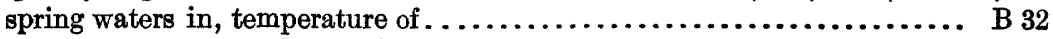

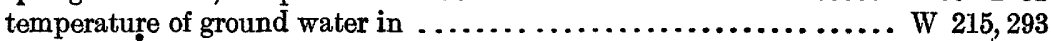

well records for .......................... W 57, 149, 293; B 264, 298

Iron, field tests for . ......................................... 151

Iron in ground water.. W 254, 256, 259, 273, 338, 341, 398, 399; MR 1911 II d, 1916; S 2

Irrigation, artesian water for. ................................ $5 \mathrm{c}$

artesian water for, development of, about $1890 \ldots \ldots \ldots \ldots \ldots$. A 11 II c, 13 II a

early developments of ................................ A 16 II e

early investigations of. ................................. A 11 II $\mathrm{c}$

in Algeria.......................................... 136; 11 Ir $c$

in Arizona................................................. 425 a

in California................. W 137, 138, 139, 142, 219, 222, 225, 398

in Colorado .......................................... 240

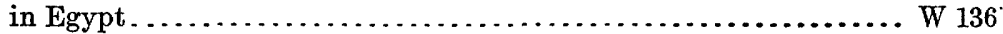

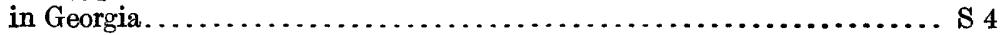

in Great Plains . . . . . . . . . . . . . . . .

in Minnesota.................................................. 25 .

in Montana............................................ $400 \mathrm{~b}$

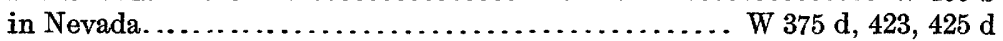

in New Mexico................................. W 158, 425 a

in North Dakota............................. A 17 m g; M 25

in South Dakota......................... A 16 II e, 17 II g, 18 Iv c

in Texas................................ $13,71,375 \mathrm{~g}$

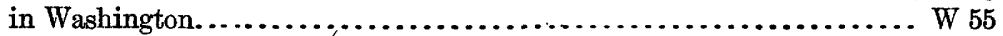

effect of, on quality of ground water...................... 58

on water table..................W 18, 58, 142, 155, 219, $345 \mathrm{~h}, 375$ a

ground water for, cost of. See under Pumping and Wells.

ground water for, development of, about $1890 \ldots \ldots \ldots \ldots \ldots$ A 11 II c, 13 III a

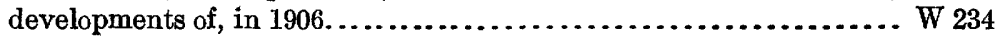

early developments of. ......................... $13 \mathrm{mb}$ b, $16 \mathrm{Ir} \mathrm{e}$

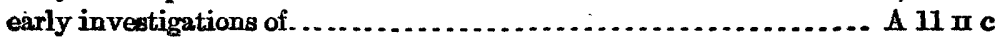


Irrigation, ground water for, flood waters in connection with ......... W 320, 343 in Arizona....................... W 104, 136, 320, $375 \mathrm{~b}, 425 \mathrm{a} ; \mathrm{A} 16$ II e

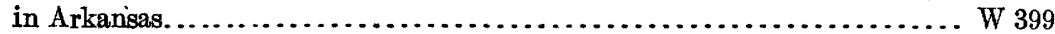

in California................................................ 17,

$45,58,59,60,89,116,137,138,139,142,181,219$,

$222,225,278,345 \mathrm{~h}, 375$ a, 398, 400 e; A 16 Ir e in Colorado..................... W 9, 240; A 16 п e, 16 II f, 21 rv c, 22 rv c in Egypt................................................ 136 in foreign countries. .................................... A 13 nr b in Georgia............................................ W 341 ; 4 in Great Plains................... W 5, $8,345 \mathrm{~b} ; \mathrm{A} 16$ II f, 21 rv c, 22 Iv c in Hawaii ............................................ 318,336 in High Plains................................. A 21 rv c, 22 Iv c in Idaho ........................................ 16 п e; GF 103 in India............................................. 11 In in Kansas........... W 6, 8, 153, 258, 345 a b; A 16 II e, 16 II f, 21 IV c, 22 Iv c

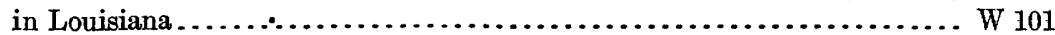
in Montana....................................... W 345, $400 \mathrm{~b}$ in Nebraska........................................... 12 , $29,184,216,425$ b; A 16 I e, 16 In f, 19 rv c, 21 rv c, 22 Iv c; P 17 in Nevada................................ W 365, $375 \mathrm{~d}, 423,425 \mathrm{~d}$ in New Mexico...................................... 10 , $140,141,188,260,275,343,345 \mathrm{c}, 380,422$ 425 a; B 618 ; A 16 II e, 21 Iv c, 22 Iv c in North Dakota ................................. A 16 II e in Oklahoma................ W 148, $345 \mathrm{~b}, 345 \mathrm{~d} ; \mathrm{A} 16 \mathrm{II} \mathrm{e,} 21 \mathrm{IV}$ c; $22 \mathrm{IV} \mathrm{c}$ in Oregon ............................ W 220, 231; A 16 пा e; GF 103 in South Dakota............................. A 16 II e, $17 \mathrm{II} \mathrm{g}, 18$ Iv c in Texas................ W 13, 71, 154, 191, $375 \mathrm{~g}$; A 16 Ir e, $21 \mathrm{rv}$ c, 22 iv c in Utah................................ W 217, 277, 333; A 16 I e in Washington .......................... $55,316,425$ e; A 16 Ir e in Wyoming............................. W $425 \mathrm{~b} ; \mathrm{A} 21 \mathrm{rv} \mathrm{c}, 22 \mathrm{rv} \mathrm{c}$

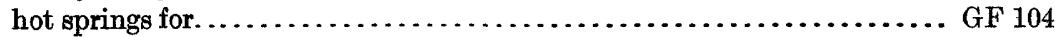
quality of ground water for.........W 260, 274, 320,333, 335, 343, $375 \mathrm{~g}, 398,399$ rice, ground water for............................. 101, 399 subsurface........................................ A 13 III b supplementary, ground water for......................... 275,320 temperature of ground water for............................. 398

Isochlors, in Connecticut................................ 144, 232

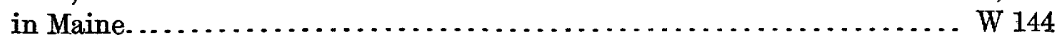

in Massachusetts.............................................. W 144

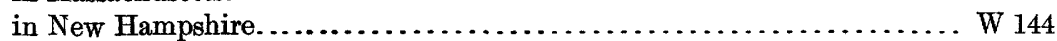

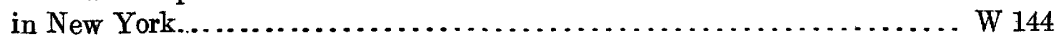

in Rhode Island........................................... W 144

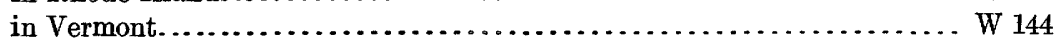

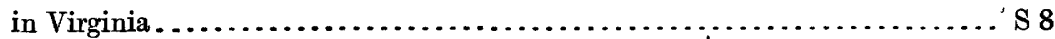

Italy, bibliography of ground water in............................. 163 quality of ground water in $\ldots \ldots \ldots \ldots \ldots \ldots \ldots \ldots \ldots \ldots \ldots \ldots \ldots \ldots \ldots \ldots$

J.

Jackson, D. D., Normal chlorine in New York and New England........... . W 144 Jackson coal measures, water in, in Michigan...................... W 30 
Jackson formation, water in, in Arkansas......................... W 399 water in, in Florida.................................... 319,128

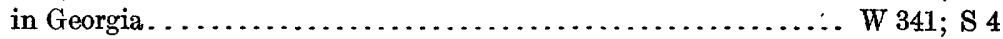

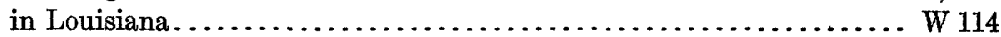

in Mississippi. . . . . . . . . . . . . 159

Jameco gravel, water in, in New York......................... P 44

Japan, bibliography of ground water in........................... 163

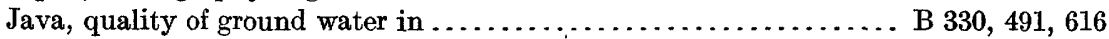

Jefferson City limestone, water in, in Missouri........................ W 195

Johnson, B. L., Bibliography of ground-water literature in $1905 \ldots \ldots \ldots \ldots$. W 163

Coastal Plain of North Carolina. ........................... S 5

Johnson, D. W., Law relating to ground water....................... W 122

Johnson, G. A., Purification of public water supplies..................W 315

Johnson, H. R., Water resources of Antelope Valley................. W 278

Johnson, L. C., Underground waters of Mississippi............... W 102, 114

Underground-water resources of Mississippi.....................W 159

Johnson, W. D., The High Plains and their utilization.......... A 21 iv c, 22 Iv c

Joints, artesian water in............................ W 160, 232; B 319

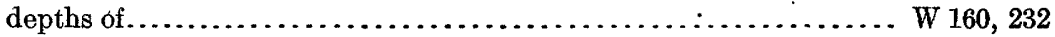

drilling in relation to.................................. W 223, 256

in crystalline rocks, water in . . . . . . . . . . . . . . . 232

in gneiss, water in ....................................... 223

in granite, water in...................................... 223

in schist, water in ......................................... 223

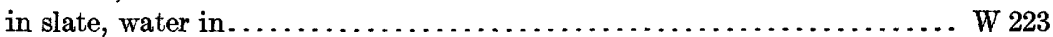

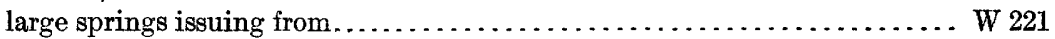

pollution of water in . . . . . . . . . . . . . . . . . . . . 258

springs in relation to................................. 221,232

water in .............. 114, 145, 160, 221, 223, 232, 254, 258; B 319; GF 149

Jordan sandstone, water in, in Iowa ............................ W 293 water in, in Minnesota ............................... 256; GF 201

See also Cambrian sandstones and Potsdam sandstone.

Jurassic formations, water in, in Arizona........................... W 380

water in, in Kansas....................................... 16 п $\mathrm{f}$

in Nebraska...................................... 16 II $\mathrm{f} ; \mathrm{GF} 108$

in New Mexico...................................... W 380

in South Dakota....................... P 32; GF 107, 127, 164

in Utah.................................... $380 ; \mathrm{B} 628 ; \mathrm{P} 56$

in Wyoming............. P $32,56,65$; B 471, 543; GF 107, 108, 127, 150

See also specific formations.

Juvenile waters.

W $160 ;$ B $319,330,491,616$

K.

Kansas, areas, all of State........................... W 273; A 11 II $c$ areas, Allen County....................................... B 298

Arkansas Valley................................. 153, 345 a

central.............................. A 21 Iv c, 22 Iv c; P 32

Garden City and vicinity ......................... 140, 153

Greeley County ...................................... B 264

Independence quadrangle............................ GF 159

Iola quadrangle.................................... B 238

Joplin district............................ W 145; GF 148

Leavenworth quadrangle.............................. G 206

Meade artesian-basin............................W 6; A 21 IV c

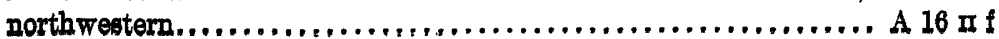




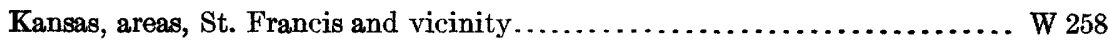
southwestern............................................. W 6 western.............................. A 21 rv c, 22 rv c; P 32 Wichita and vicinity .................................... W 345 a artesian water in............. W 6, 273; A 11 II c, 16 II f, 21 rv c; P 32; GF 148 bibliography of ground water in..................... W 57, 120, 149, 163 blowing wells in ........................................ 16 n $\mathrm{f}$

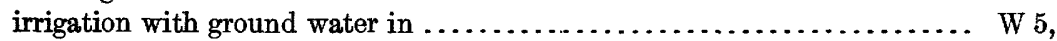
$6,153,258,345 \mathrm{a}, \mathrm{b} ; \mathrm{A} 11$ Ir c, 16 Ir e, 16 Ir f, 21 rv c, 22 rv c irrigation with windmills in. $\mathrm{W} 8$. mineral waters in.................... B 32; MR 1883-1916; GF 148, 159 public water supplies in...................... W 273; P 32; GF 148 pumping in, cost of.................................. 153, 258, 345 a pumping tests in................................ 140.153, 258, 345 a quality of ground water in................................. 6 , $145,153,273,345$ a; B 32, 330, 491, 530 b, 606, 616; P 32; GF 206

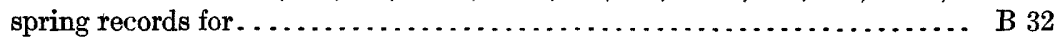

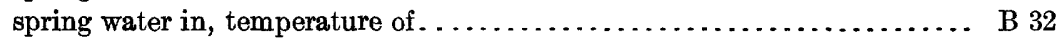
underflow measurements in ............................. 153, 258 water-table in, fluctuations of. ............................... 153 well records for............................. 57,$149 ;$ B 131, 264, 298 wells in, construction of................................ 258,345 a cost of.......................................... 345 a; A 11 II c

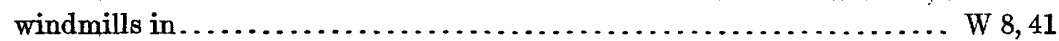
Karst water. See Limestone.

Katamorphism, zone of, ground water in relation to ............... M 47

Keith, Arthur, Mount Mitchell folio, N. C.-Tenn.................... GF 124

Pisgah folio, N. C.-S. C............................... GF 147

Roan Mountain folio, Tenn.-N. C........................... GF 151

Washington quadrangle, D. C.-Md.-Va........................ GF 70

Kelton, F. C., Geology and water resources of Sulphur Spring Valley, Ariz... W 320

Kentucky, areas, all of State.............................. 102, 114 areas, bluegrass region...................................... 233

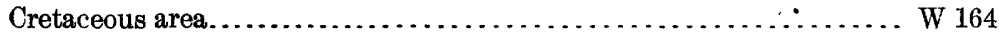

Estill County......................................... B $661 \mathrm{~d}$

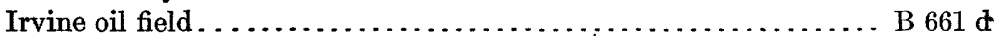

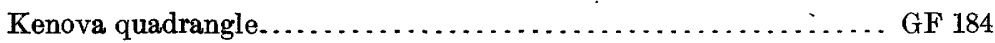

Metcalfe County ....................................... B 298

Middlesboro-Harlan region........................... W 110

north-central......................................... W 233

west of Tennessee River.................................. 164

artesian water in....................................... 164,233

bibliography of ground water in.............. W 57, 114, 120, 149, 163, 164, 233

law relating to ground water in................................. 152 mineral waters in....................... W 164, 233; B 32; MR 1883-1916 public water supplies in.................................. W 233 quality of ground water in .................................... 102 , $164,233.364 ;$ B 32, 330, 491, 606, 616, 661 d; GF 184 spring records for ........................................ $32,661 \mathrm{~d}$ spring water in, temperature of $\ldots \ldots \ldots \ldots \ldots \ldots \ldots \ldots \ldots \ldots \ldots \ldots \ldots \ldots \ldots \ldots$ B 32 well records for................................. W 57, 149; B 298 wells in, construction of . . . . . . . . . . . . . . . 164

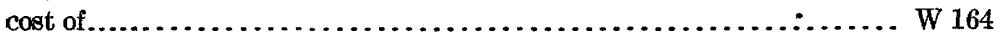


Keokuk limestone, water in, in Illinois . . . . . . . . . . . . . . . . B 438 water in, in Missouri . ............................... W 195; B 438

Keweenawan formations, water in, in Wisconsin................... S 9

Keyes, C. R., Geology and underground water conditions of the Jornada del Muerto, N. Mex............................................... W 123

Kinderhook formation, water in, in Illinois......................... B 438 water in, in Iowa............................................... 293

in Missouri................................................ 438

Kindle, E. M., Water resources of the Catatonk area, N. Y................ W 145

Niagara folio, N. Y.....................................FF 190

Watkins Glen-Catatonk folio, N. Y.......................... GF 169

King, F. H. , Movements of ground water...................... A 19 п b

King's aspirator, description of............................... W 67

Kingsbury conglomerate, water in, in Wyoming..................F 141, 142

Knapp, G. N., Underground waters in New Jersey.................... W 114

Philadelphia folio, Pa.-N. J.-Del........................... GF 162

Knobstone formation, water in, in Indiana......................... 114

Kokomo limestone, water in, in Indiana.......................... W 254

Kümmel, H. B., Franklin Furnace folio, N. J........................ GF 161

Passaic folio, N. J.-N. Y................................... GF 157

Philadelphia folio, Pa.-N. J.-Del.......................... GF 161

Raritan folio, N. J.................................... GF 191

Trenton folio, N. J.-Pa.................................... GF 167

LaForge, Laurence, Ellijay folio, Ga.-N. C.-Tenn.................... GF 187

Massachusetts, well and spring records for..................... W 102

Water resources of the central and southwestern highlands of New Jersey .. W 110

La Motte sandstone, water in, in Missouri....................... W 195

Lacustrine deposits, artesian water confined under..................... W 425 a

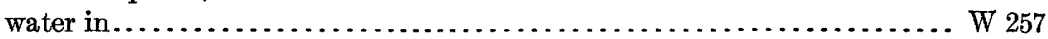

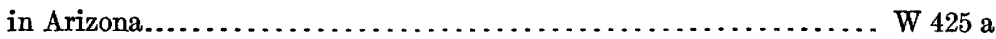

in Illinois................................................ 105

in Indiana.................................................. 105

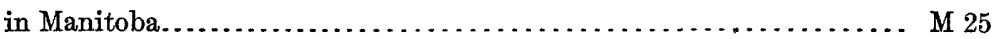

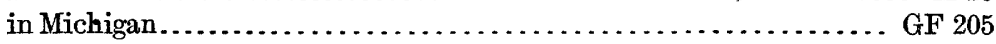

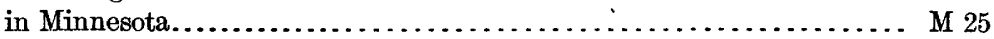

in Montana........................................ $221,400 \mathrm{~b}$

in North Dakota....................................... 25

in Oregon ................................................ 220,231

in Utah........................................ $217,277,333$

in Washington............................................... 425

in Wisconsin ............................................ 114 ; 9

Lafayette formation, water in, in Arkansas................. $399 ;$ P 46

water in, in Atlantic Coastal Plain............................... 138

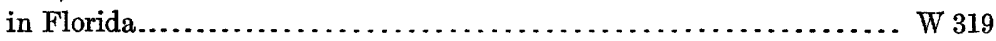

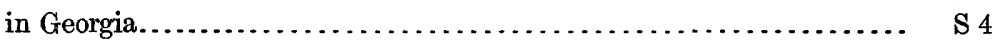

in Kentucky.............................................. 164

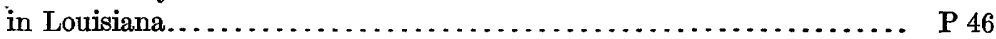

in Maryland ....................................... 13, 23, 152

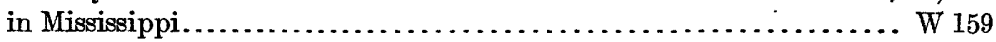

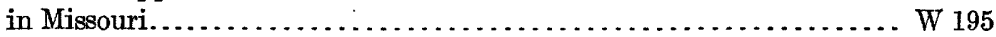

in North Carolina........................................... S 5

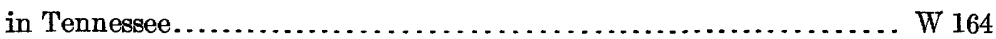

in Virginia..................................... 13,23 ; 8 
Lagrange formation, water in, in Kentucky

water in, in Missouri...................................... W 195

in Tennessee............................................... 164

Lake beds. See Lacustrine deposits.

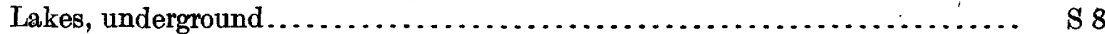

Lakota sandstone, water in, in Montana............................ GF 138

water in, in Nebraska................................. GF 85, 108

in South Dakota............. A 21 Iv b; P 32, 65; GF 85, 107, 108, 128, 164

in Wyoming............... A 21 iv b; P 32, 65; GF 107, 127, 128, 150

Lamination planes, water in............................... W 319

Lance formation, water in, in North Dakota.................. B 575; GF 181 water in, in South Dakotá..................................... B 575

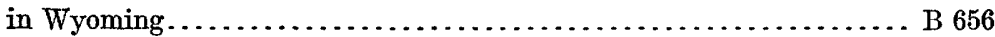

Landes, Henry, Underground waters of Washington................... W 111

Lane, A. C., Flowing wells and municipal water supplies in the southern

peninsula of Michigan................................ W 182, 183

Lower Michigan mineral waters............................. W 31

Water resources of the lower peninsula of Michigan................ W 30

Lansing formation, water in, in Kansas.............................. 206

water in, in Missouri........................................ 206

Laramie formation, water in, in Colorado.............. M 27; P 32, 52; GF 71

water in, in Kansas................................... P 32

in Nebraska................................... P 32; GF 87

in South Dakota................................ W 227; P 32

in Wyoming........................................ P 32

Larrison, G. K., Report of Water Commission of Hawaii. . . . . . . . . . . . . . . S 12

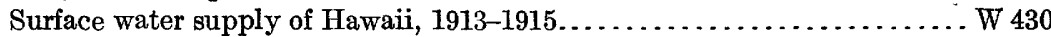

Surface water supply of Hawaii, $1915-16 \ldots \ldots \ldots \ldots \ldots \ldots \ldots \ldots \ldots \ldots$ W 445

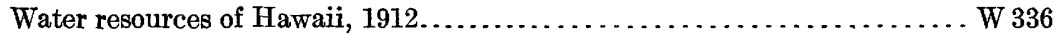

Lathas for lifting water ...................................... W 1

Lava, artesian water in . . . . . . . . . . . . . . . . . . . . . . W 4; B 199; S 12

water in ...................................... 110, 160; B 319

in California............................ W 219, 375 a; GF 138

in Connecticut.........................

in Hawaii.......................................... 77 . 12

in Idaho......................................... W $54 ;$ B 199

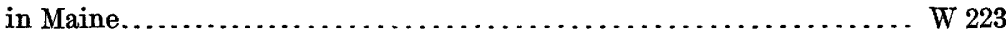

in Nevada............................................ W 365

in Oregon................................. W 220, 231; B 252

in Texas....................................... F 64

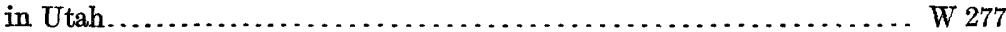

in Washington.......................... 4, 55, 118, 316, 425 e; G F 106

Law, natural, of flow of ground water................. W 67,140; A 19 II b

natural, of flow of water into wells............................ W 140

of movement of ground water............................... 47

prohibiting flooding of oil wells............................... W 257

relating to artesian water in Hawaii.......................... S 12

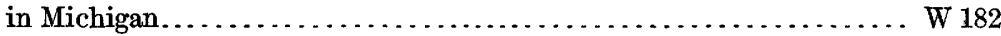

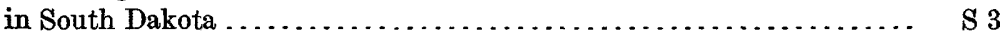

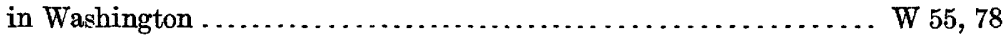

relating to ground water, in Arkansas........................... 152

in California........................................ 122,152

in Colorado....................................... 122

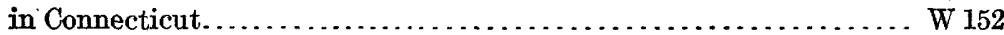


Law, relating to ground water, in Florida........................ W 152

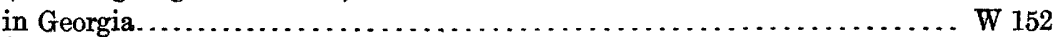

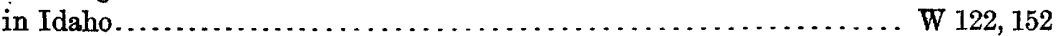

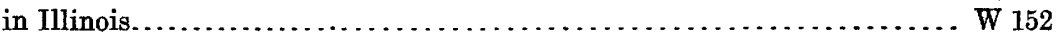

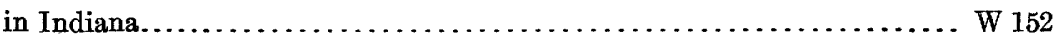

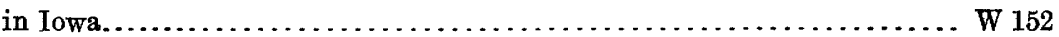

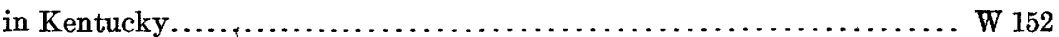

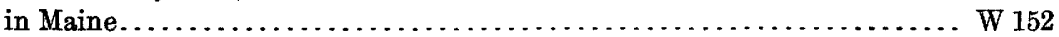

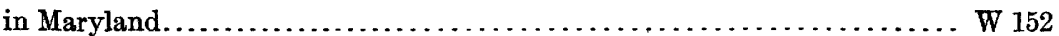

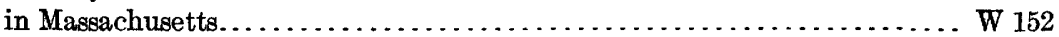

in Michigan.............................. $122,152,182,183$

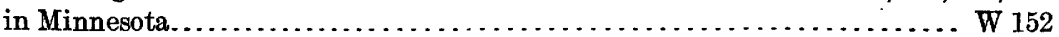

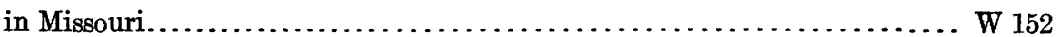

•in Nebraska................................... W 122, 152

in Nevada.................................... W 122, 152

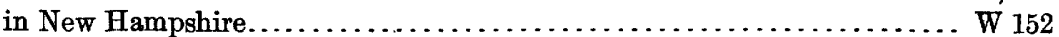

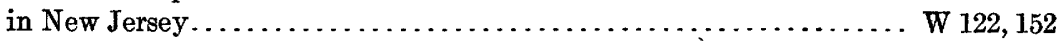

in New Mexico........................................ W 152

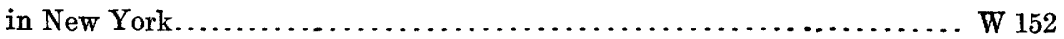

in North Carolina.................................... W 152

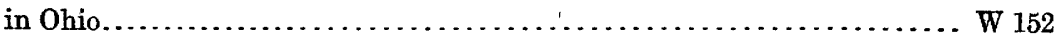

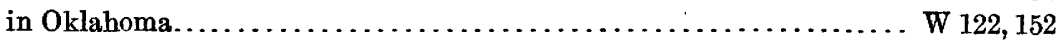

in Oregon .......................................... 122,152

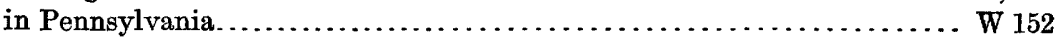

in Rhode Island . . . . . . . . . . . . . . . . . . . . . . . . . . . . . . . 152

in South Dakota............................... W 122; S 3

in Tennessee............................................ 152

in Texas.................. 152

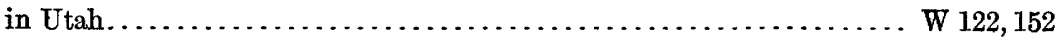

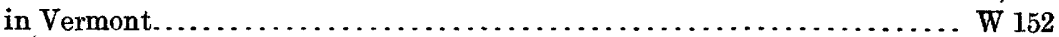

in Virginia ................... 152

in Washington................................ $55,78,122,152$

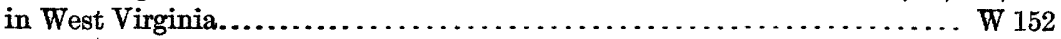

in Wisconsin........................................... 122

in Wyoming...................................... 122,152

relating to pollution of ground water....................... 122,152

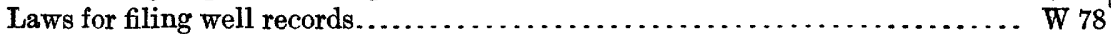

Lawson, A. C., San Francisco folio, Cal............................. GF 193

Lead in ground water.......................... 254,$399 ; \mathrm{B} 47,606$

Leaks in artesian wells, methods of detecting.................. W 118; A 5 c

Lee, C. H., Ground-water resources of Indian Wells Valley, Cal ............ S 7

Subterranean storage in San Bernardino Valley, Cal................ S 6

Water resources of a part of Owens Valley, Cal.....................W 294

Lee, W. T., Geologic reconnaissance of part of western Arizona ............ B 352

Geology and water resources of Owens Valley, Cal..................W 181

Underground waters of Gila Valley, Ariz........................ W 104

Underground waters of Salt River Valley, Ariz ................... W 136

Water resources of Beaver Valley, Utah........................ W 217

Water resources of Rio Grande valley in New Mexico and their develop-

ment................................................. 188

Legal. See Law.

Legislation. See Law. 
Leighton, M. O., Field assay of water........................... W 151

Quality of water in Susquehanna River basin.................... W 108

Leona formation, water in, in Texas.................... A 18 II b; GF 64

Leonard, A. G., Bismarck folio, N. Dak......................... GF 181

Leverett, Frank, Ann Arbor folio, Mich............................. GF 155

Danville folio, Ill.-Ind................................... 67

Flowing wells and municipal water supplies of southern peninsula of Mich-

igan.............................................. 182,183

Flowing wells in northern Michigan......................... 160

Illinois glacial lobe........................................ 38

Underground waters of Illinois, Indiana, and Ohio ................W 114

Water resources of Illinois.............................. $17 \mathrm{II} \mathrm{h}$

Water resources of Indiana and Ohio....................... A 18 iv b

Wells of northern Indiana................................... W 21

Wells of southern Indiana................................. W 26

Lexington limestone, water in, in Kentucky...................... W 235

Lifting devices. See Pumps and other lifting devices.

Limestone, caverns formed by ground water in............W 233, 255, 259; S 4 drainage into wells in...................................... 258 pollution of water in .......................... $233,255,258$; S 3 solution channels in ............................. 233, 255, 259 turbidity of water in ........................................... S 3

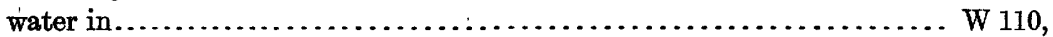

$113,114,148,158,223,232,233,254,255,256,257$, $258,259,293,319,374 ;$ A 5 c; GF 170,$173 ;$ S $3,9,10$

Lindgren, Waldemar, Boise folio, Idaho:............................ GF 45

Clifton folio, Ariz........................................ 129

Colfax folio, Cal......................................... GF 66

Marysville folio, Cal ......................................... 17

Nampa folio, Idaho-Oreg...................................FF 103

Silver City folio, Idaho.................................. GF 104

Truckee folio, Cal........................................... 39

Water resources of Molokai, Hawaiian Islands..................... W 77

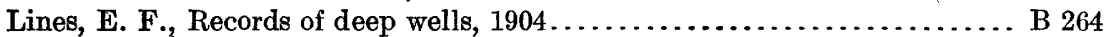

Lippincott, J. B., Development and application of water near San Bernardino,

Colton, ind Riverside, Cal........................... W 59, 60

Storage of water on Kings River, Cal........................ W 58

Water problems of Santa Barbara, Cal......................... W 116

Lissie gravel, water in, in Texas.............................. W 335

Literature on ground water. See Bibliography.

Lithium in ground water................. W 160, 259, 338; B 47; MR 1911 in n

Little, H. P., Tolchester folio, Md................................... 204

Live stock, quality of water for............................... 335,343

L'loyd, E. R., Lignite field of northwestern South Dakota................... B 627

Lloyd sand, water in, in New York.......................... P 44

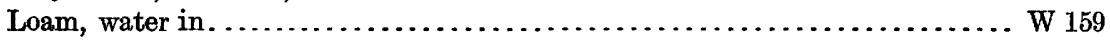

Locating water in deserts........................ W 224, 423; B 308

Loess, turbidity of ground water due to......................... S 3

water in, in Illinois.................... W 164; GF 105, 188, 195; S 3

in Indiana.......................................... 105

in Iowa...................................... W 293; GF 156

in Kentucky.......................................... 164

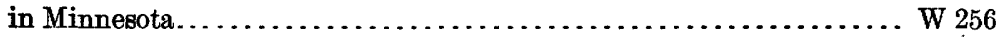


Loess, water in, in Mississippi................................. W 159

in Nebraska......................................... 156

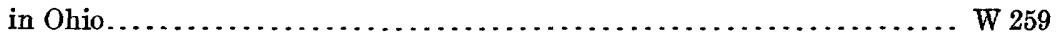

in South Dakota............................................ 156

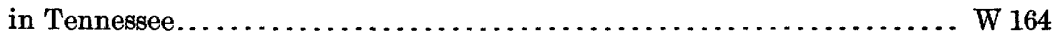

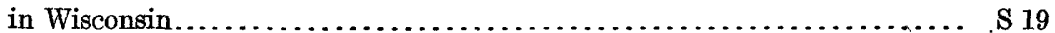

Logan sandstone, water in, in Ohio.......................... W 114

Logarithms for recomputing water analyses...................... W 293

Long Island. See New York.

Loss of ground water. See Discharge of ground water.

Lost rivers..................................... A 18 Iv b, 21 rv c

Louisiana, areas, all of State................................... W 114

areas, Coastal Plain....................................... B 282

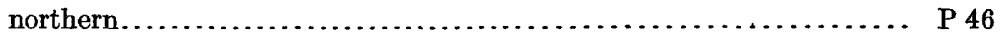

southern............................................. W 101

artesian water in ..................................... $101 ;$ P 46

bibliography of ground water in................... W 114, 120, 149, 163

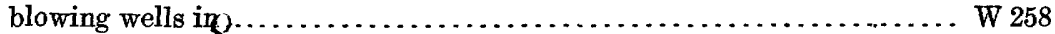

mineral waters in ....................... W 114; B 32; MR 1883-1916

public water supplies in............................ W 101; P 46

quality of ground water in................ W 101; P 46; B 330, 491, 616

rice irrigation with ground water in......................... W 101

salt water in ........................................... $530 \mathrm{~b}$

salt water in oil fields of................................. B 282

spring records for.......................................... B 32

well records for................................ W 149; B 264, 298

wells in, construction of.................................. P 46

cost of..................................... P 46; B 282

Louisiana limestone, water in, in Missouri........................ W 195

Lower Magnesian limestone, water in, in Illinois............ A $17 \mathrm{~m} \mathrm{~h}$; GF 81

water in, in Wisconsin..................................... 14

See also New Richmond sandstone, Oneota limestone, Prairie du Chien

- group, and Shakopee dolomite.

Lucas dolomite, water in, in Michigan............................. GF 205

Lupton, C. T., Anticlines in southern part of Big Horn Basin, Wyo.......... B 656

Bull Mountain coal field, Mont............................ B 646

Geology and coal resources of Castle valley in Carbon, Emery, and Sevier

counties, Utah.................................... B 628

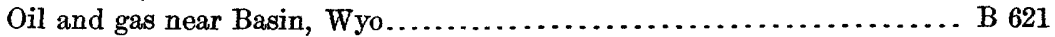

Oil and gas near Green River, Utah......................... B 541 d

Lysimeters for measuring absorption of water....................... W 155

M.

McCallie, S. W., Underground waters of Georgia.................... W 114

Underground waters of Georgia............................... S 4

Well and spring records for Georgia. . . . . . . . . . . . . . . . . . 102

Well contamination at Quitman, Ga.......................... W 110

McGee, W J, Potable waters of eastern United States ............... A 14 II a

McGlashan, H. D., Surface water in California, 1912.................. W 331

Water resources of California, Part III . . . . . . . . . . . . . . . . . W 300

McLouth, C. D., Flowing wells and municipal water supplies of southern

Michigan.............................................. 183

Magmatic waters........................... W 160; B 319, 330, 491, 616 
Magnesium in ground water ...... W 254, 259, 273, 338, 341, 343, 398; MR 1911 II $\mathbf{n}$ Magnetism in ground water................................... 258 ; 8 Magothy formation, water in, in Maryland ................... $152,182,204$ Mahoning sandstone, water in, in Pennsylvania.................. GF 123

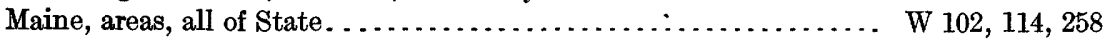

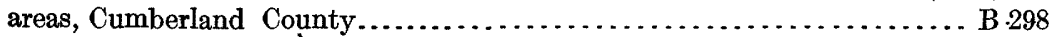

Eastport quadrangle................................... GF 192

near Augusta . ........................................... W 145

Penobscot Bay quadrangle.......................... GF 149

Portsmouth-York region............................... W 145

Rockland quadrangle. . . . . . . . . . . . . . . . . . . . . . 158

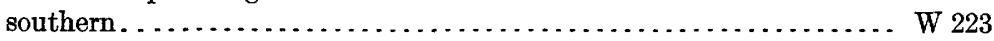

artesian water in....................... W 145, 223, 258; GF 149, 158

bibliography of ground water in.................... W 114, 120, 163

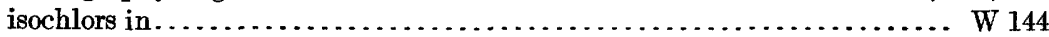

law relating to ground water in............................ W 152 mineral waters in.................. W 258; B 32; MR 1883-1916; GF 149 pollution of ground water in . . . . . . . . . . . . . . . . . . . . . . . W 223 public water supplies in........................ W 114, 223; GF 149 quality of ground water in .. W 102, 114, 223, 258, 364; B 32, 330, 491, 616; GF 149

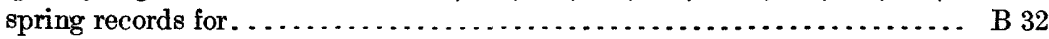
spripg water in, temperature of ............................ B 32 well records for............................ W 57, 149, 223; B 264, 298 wells in, construction of ..................................... 223

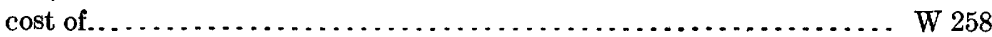

Malt making, quality of water for.................. W 233, 254, 341, 398 Manganese in ground water ..................... W 338; B 47; MR 1911 in $n$ Manitoba, ground water in, quality of . . . . . . . . . . . . . . . 25 southern. . . . . . . . . . . . . . 25

Manufacturers of well drills...................................... W 257

Maps, representation of wells and springs on..................... W 160

Maps showing absorption areas in New Mexico..................... W 158 absorption areas in South Dakota.......................... A 21 iv b in Texas .................................. W $375 \mathrm{~g} ; \mathrm{A} 21$ vII in Wisconsin ................................ A 17 II $\mathrm{h} ; \mathrm{M} 38$ in Wyoming ..................................... 21 iv $b$ areas irrigated with ground water in Arizona ................. W 425 a in California.......................W 137, 138, 139, 142, 219, 225 areas of artesian flow in Alabama ............................ W 114 in Arizona. . . . . . . . . . . . in Arkansas..................................... W $399 ; \mathbf{P} 46$ in California....... W 89, 137, 138, 139, 142, 213, 219, 222, 225, 278, 398; S 6 fluctuations of .................. W 137, 138, 139, 142, 213, 219; S 6 in Colorado.................. W 240; P 32, 52; GF 36, 58, 68, 71, 135 in Florida........................................... W 114 in Georgia...................................... W 114, 341 ; $\mathbf{S} 4$ in Illinois ...................................... 17 II $\mathrm{h} ; \mathrm{M} 38$

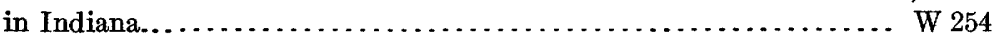

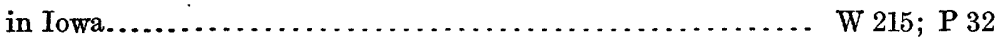

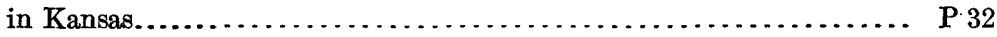
in Maryland......................................... 23 in Michigan............................ 182, 183; GF 155, 205 in Minnesota................................... W 256; GF 117 in Mississippi...................................... W 159 
Maps showing areas of artesian flow in Montana

GF 128

in Nebraska W 215; A 19 Iv c; P 17, 32; GF 85

in Nevada. W $425 \mathrm{~d}$

in New Mexico W 158

in North Dakota

A 17 II g; GF 117,168

in Ohio W 259

in South Dakota W 90, 215; A 17 II g; P 32, 65; GF 85, 96, 97, 99, 100, 107, 108, 113, 114, 128, 164, 165

in Texas W 335,375 g; A 21 vII

in Utah. W $157,277,333$

in Virginia GF $23 ; \mathrm{S} 8$

in Wyoming P 32,$63 ;$ GF $107,127,128,150,173$ areas of black alkali water................................ W 320 areas of colored ground water............................. W 240 areas of ground-water discharge................... W $375 \mathrm{~d}, 423,425 \mathrm{~d}$ contours of oil-water and gas-water surfaces.................. B 658 contours of water table W $67 ;$ A 19 in b

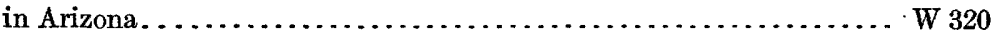
in California........ W 59, 137, 138, 139, 142, 219, 222, 294, $345 \mathrm{~h}, 398,400$ e in Montana W 343; GF 207 in New Mexico. in New York........................................ P 44 in Washington............................................. 425 depths to water-bearing formations in Colorado.. P 32, 52; GF 36, 58, 68, 71, 135 in Iowa. W 215; GF 156

in Kansas. B 138; GF 136 in Maryland. GF 117 in Minnesota. GF 128

in Montana $\mathrm{W} 12 ; 215 ; \mathrm{GF} 85,156 ; \mathrm{P} 32$ in Nebraska. in North Dakota............................. A $17 \mathrm{~m} \mathrm{g;} \mathrm{GF} 117$ in South Dakota........ W 34, 90,215, 227, 428; A $17 \mathrm{Ir} \mathrm{g,} 21 \mathrm{rv} \mathrm{b;} \mathrm{P} \mathrm{32;} \mathrm{GF}$ $85,97,99,100,107,108,113,114,127,128,156,164,165$ in Texas................................... 21 vI in Wyoming................ A $21 \mathrm{IV} \mathrm{b;} \mathrm{P} \mathrm{32;} \mathrm{GF} \mathrm{107,} \mathrm{127,} \mathrm{128,} \mathrm{150,} 173$ depths to water table in Arizona ................... W 320,375 b, 425 a

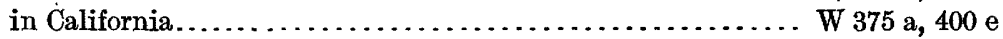
in Connecticut..................................... 374,397

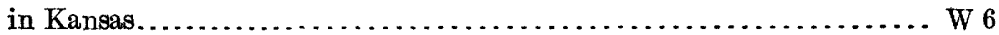
in Nebraska............................... 425 b; A 19 rv c; P 17 in Nevada.................................. W $375 \mathrm{~d}, 423$ in New Mexico............. W 275, 343, 345 c, 422, 425 a; B 618; GF 207

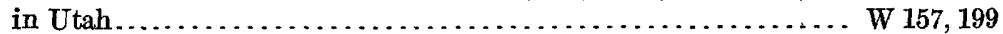
in Wyoming. . . . . . . . . . . . . . . . . $425 \mathrm{~b}$ desert watering places in Arizona............................. W 380 in California . . . . . . . . . . . . . . . . . . . . . . . . . $224 ; \mathrm{B} 308$ in Nevada................................ 224,$365 ;$ B 308 in New Mexico..................................... W 343, 380

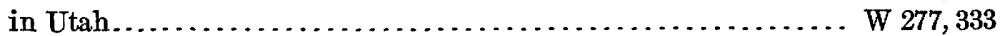

faults in relation to carbonated springs. ...................... W 338 to flowing wells ................................... 18 r b to ground-water levels . . . . . . . . . . . . . . . . . . . . . . . $345 \mathrm{~h}$

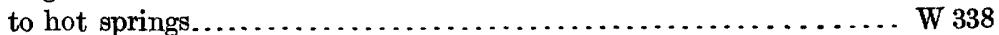

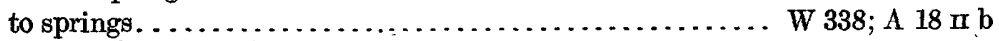

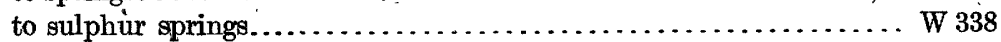


Maps showing ground-water divide on Long Island, N. Y

in Colorado

P 52; GF 135, 186

in Illinois.

W 164

in Kansas.

A 21 IV c

in Kentucky

W 164

in Louisiana

P 46

in Michigan

GF 205

in Nebraska

GF 85

in North Dakota.

A 17 II g; GF 117

in South Dakota..... W 90, 227; A 17 II g; GF 85, 96, 97, 99, 100, 108, 114, 165

in Tennessee.

W 164

in Wisconsin.

localities where irrigation with artesian water is practicable.

A 17 II $g$ movement of ground water.

$\mathrm{W} 67 ; \mathrm{A} 19 \mathrm{Ir} \mathrm{b}$ quality of ground water, bicarbonates in Arizona................. W 320

black alkali in Arizona . . . . . . . . . . . . . . . . . . . . . . . W 320

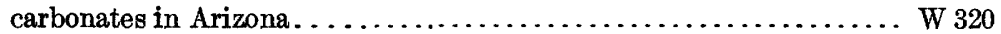

chlorine in Arizona......................................... 320

in Connecticut............................. 144, 232

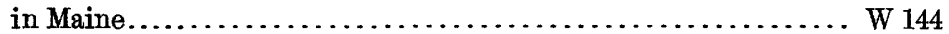

in Massachusetts................................... W 144

in New Hampshire................................. W 144

in New Mexico................................... 275

in New York. . . . . . . . . . . . . . . . . . . . . . . . . . . . 144

in Rhode Island................................. W 144

in Vermont......................................... W 144

in Virginia. . . . . . . . . . . .

hardness in Arizona................................... W 320

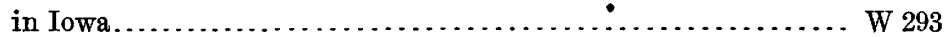

in Michigan ......................................... 31

in Minnesota....................................... W 256

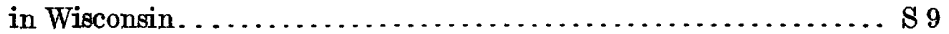

potability in North Carolina............................. S 4

relation to depth in Minnesota.......................... W 256

saline shallow water in Michigan...................... W 30,31

sulphates in Arizona................................. W 320

in California................................... W 398

in New Mexico................................... W 275

total solids in Arizona................................. W 320

in California.............................. W 137, 138, 139

relation of glacial drift to ground water............. W 21; A 18 Iv b; M 38

sink holes.............................................. W 319

structure contours of water-bearing formations in Colorado. . . . . . P 32, 52

in District of Columbia . ......................... GF 70, 152

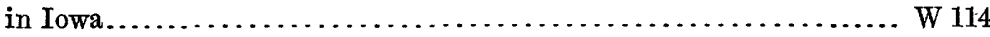

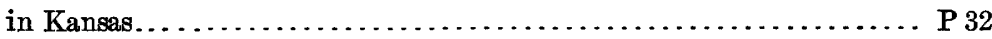

in Louisiana................................... P 46

in Maryland................................ GF 24,70,152, 182

in Nebraska.................................... W 12; P 32

in North Dakota. . . . . . . . . . . . . . . . . . . . . . . . . . . . . . 168

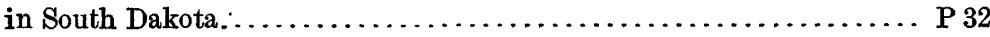

in Texas.................................. W 335; A 21 vII

in Virginia..................................... GF 23,70

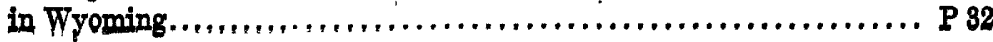


Maps showing temperature in deep wells, rates of increase in......... A 18 IV c temperature of ground water in Michigan.................. W 182, 183 thickness of water-bearing deposits................ W 254, 256, 259; GF 205 vegetation in relation to water supplies in Arizona............... W 320

in Nevada....................................... W 423

in New Mexico....................................... W 343

water table in California, fluctuations of................ W $345 \mathrm{~h}, 400 \mathrm{e}$

watering places on routes of travel in Arizona ..................W 380

in California.................................. W 224; B 308

in Nevada................................ W 224, 365; B 308

in New Mexico................................... W 343, 380

in Utah....................................... $27 \dot{\ldots}, 333$

yields of flowing wells............................... 18 IV $\mathrm{c}$

Maquoketa shale, ground water in relation to, in Wisconsin............. S 9

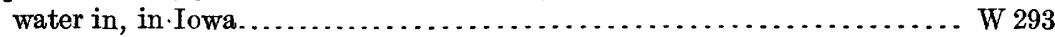

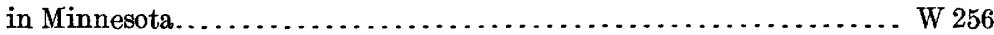

Marl, water in, on Great Plains.............................. A 16 in $f$

Marshall formation, water in, in-Michigan.................... 30, 31, 114

Martin, G. C., Accident-Grantsville folio, Md.-Pa.-W. Va................. GF 160

Water resources of the Accident and Grantsville quadrangles, Md......... W 110

Water resources of the Frostburg and Flintstone quadrangles, Md.-W. Va.. W 110

Water resources of the Pawpaw and Hancock quadrangles, W. Va.-Md.-Pa.W 145

Martin, Lawrence, Mineral resources of Johnstown, Pa.................. B 447

Martin, W. F., Water resources of Hawaii, 1909-1911................... W 318

Maryland, areas, Accident quadrangle...................... W 110; GF 160

areas, all of State........................................ W 114

Anne Arundel County.................................. B 298

Choptank quadrangle.................................. GF 182

Coastal Plain......................................... 138

Dover quadrangle.................................... GF 137

Flintstone quadrangle................................... 110

Fredericksburg quadrangle............................... GF 13

Frostburg quadrangle...................................W 110

Grantsville quadrangle........................... W 110; GF 160

Hancock quadrangle........................... W 145; GF 179

near Baltimore................................ W 114; B 138

Nomini quadrangle............................... GF 23

Patuxent quadrangle....................................... 152

Pawpaw quadrangle............................... W 145, 179

St. Marys County ..................................... B 298

St. Marys quadrangle.................................... GF 136

Talbot County......................................... B 298

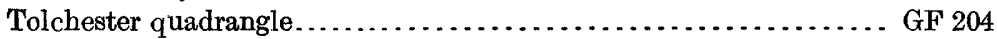

Washington quadrangle................................ GF 70

artesian water in............. B 138; GF 23, 70, 136, 137, 152, 160, 179, 182, 204

bibliography of ground water in.................... W 114, 120, 149, 163

law relating to ground water in.................................. 152

mineral waters in....................... B 32; MR 1883-1916; GF 152

public water supplies in................................. B 138

quality of ground watér in .............. W 258; B 32; GF 136, 137, 179, 204

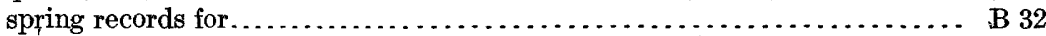

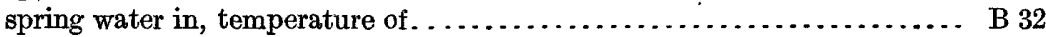

well records for................................ W 57, 149; B 298 
Massachusetts, areas, all of State.......................... W 102, 114

areas, Brookline...................................... W 374, 397

Central Lowland......................................... W 110

Hampshire County ...................................... B 298

Taconic quadrangle ...................................... W 110

bibliography of ground water in...................... W 114, 120, 163

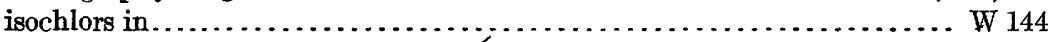

law relating to ground water in .................................. 152

mineral waters in................................... 1883-1916; B 32

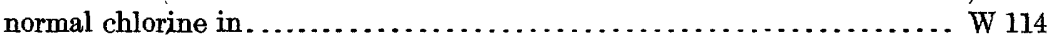

public water supplies in.............................. W 374, 397

quality of ground water in......................... W 102, 110; B 32

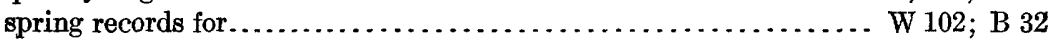

spring water in, temperature of.............................. B 32

well records for.............................. W 57, 149; B 264, 298

Matawan formation, water in, in Atlantic Coastal Plain................ B 138

water in, in Maryland ........................................... 182, 204

in New Jersey............................. 106; GF 167

in Pennsylvania........................... W 106; GF 167

Matson, G. C., Geology and ground waters of Florida................. W 319

Pollution of water in limestone.............................. W 258

Production of mineral waters............................ MR 1910-1912

Water resources of the Blue Grass region, $\mathrm{Ky} \ldots \ldots \ldots \ldots \ldots \ldots \ldots \ldots$ W 233

Mathews, E. H., Tolchester folio, Md.............................. GF 204

Maysville formation, water in, in Kentucky........................ W 233

water in, in Ohio.......................................... W 259

Mechanical water elevators................................ W 1,1 ,

Medicinal properties of water........... W 31, 164, 195, 233, 254, 259, 293, 335, 338,

341; A 14 II b; MR 1911 II n, 1913 in h

Medina formation, water in, in Indiana.......................... W 114

water in, in Ohio .................................... A 19 Iv b

Meeker, R. I., Surface water supply, $1906 \ldots \ldots \ldots \ldots \ldots \ldots \ldots \ldots \ldots \ldots$ W 209

Meeteetse sandstone, water in, in Wyoming......................... B 656

Meinzer, O. E., Artesian water for irrigation in Little Bitterroot Valley, Mont. W $400 \mathrm{~b}$

Geology and underground waters of southern Minnesota.............. W 256

Geology and water resources of Big Smoky, Clayton, and Alkali Spring

valleys, Nev.......................................... 423

Geology and water resources of Estancia Valley, N. Mex............. W 275

Geology and water resources of Sulphur Spring Valley, Ariz ........... W 320

Geology and water resources of Tularosa Basin, N. Mex............... W 343

Ground water for irrigation in Lodgepole Valley, Nebr.-Wyo.......... W 425 b

Ground water for irrigation near Wichita, Kans................. W 345 a

Ground water for irrigation on the Great Plains................ W $345 \mathrm{~b}$

Ground water in Big Smoky Valley, Nev. (preliminary) ........... W $375 \mathrm{~d}$

Ground water in Juab, Millard, and Iron counties, Utah................ W 277

Ground water in Paradise Valley, Ariz .............................. W 375 b

Ground water in Quincy Valley, Wash...................... W 425 e

Ground waters of Estancia Valley, N. Mex. (preliminary) ............. W 260

The divining rod, a history of water witching (introductory note) . . . . . . . W 416

Underground water resources of Iowa......................... . W 293

Water resources of Butte, Mont. .............................. W 345

Mendenhall, W. C., Conservation of underground water.................. W 234

Desert watering places in California and Nevada................... W 224

Development of underground waters in the central Coastal Plain of southern California. 
Mendenhall, W. C., Development of underground waters in the eastern Coastal Plain of southern California............................. W 137

Development of underground waters in the western Coastal Plain of southern California.

Fluctuations of ground-water levels in southern California.........W 251,331

Ground waters and irrigation enterprises in the foothill belt of southern

California

W 219

Ground water in San Joaquin Valley, Cal....................... W 398

Ground-water levels in southern California...................... W 213

Ground waters of San Joaquin Valley, Cal. (preliminary)............W 222

Ground waters of the Indio region, Cal., with a sketch of the Colorado Desert. W 225

Hydrology of San Bernardino Valley, Cal......................... W 142

Underground water in southern California....................... W 146

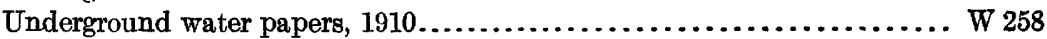

Merrill, F. J. H.; New York City folio.......................... GF 83

Mesaverde formation, water in, in Wyoming ................. B 656; GF 173

Mesozoic formations. See specific formations; also Cretaceous, Jurassic, and

Triassic.

Meters for measuring underflow

W 140,141

Methods. See specific subjects.

Mexico, bibliography of ground water in.......................... W 163

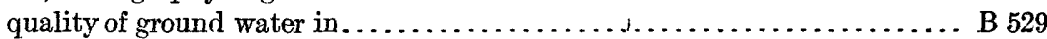

Michigan, areas, Albion and vicinity............................. 170

areas, Ann Arbor quadrangle............................... GF 155

Detroit quadrangle......................................... GF 205

Grosse Isle.............................................. W 145

northern peninsula................................. W 114, 160

southeastern................................................. 145

southern peninsula ....................... W 30,31, 102,114, 182, 183

artesian water in ........................ W 145, 160, 182, 183; GF 155, 205

law relating to........................................... 182

bibliography of ground water in.................... W $57,114,120,149,163$

blowing wells in ......................................... W 258

drainage into wells in ..................................... 145,160

fluctuations of water table in ............................... 30

laws relating to ground water in.................... W 122, 152, 182, 183

mine waters in, quality of.................................. 364

mineral waters in ................. W 114; B 32; MR 1883-1916; GF 155, 205

pollution of ground water in ............................... 30,31

public water supplies in.................... W 30, 160, 182, 183; GF 155

spring records for..................................... W $170 ;$ B 32

temperature of ground water in ...................... W $30,182,183 ;$ B 32

well construction in ................................. W $30,182,183$

well records for............................... W 57, 149; B 264, 298

Michigan formation; water in, in Michigan...................... 30,114

Midway formation, water in, in Georgia........................... W 341

water in, in Mississippi....................................... 159

Miller, B. L., Choptank folio, Md.......................... GF 182

Dover folio, Del.-Md.-N. J............................... GF 137

Patuxent folio, Md.-D. C.................................... GF 152

Philadelphia folio, Pa.-N. J.-Del............................ GF 161

St. Marys folio, Md,-Va,................................. GF 136

Tolchester folio, Md...................................... GF 204

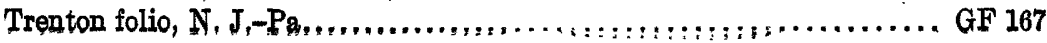


Miller, W. J.; Underground water resources of Iowa.

Millican formation, water in, in Texas.

Mine waters. See under Mines.

Mineral waters, analyses of, bibliography of

MR $1913 \mathrm{Im} \mathrm{h,} 1916$

bibliography of

MR 1905, 1913 II h, 1916; S4

chemical character of.

.B $330,491,616$

classification of.

W 293, 338

concentration of

W 341; MR 1911 II n

definition of ............... W 338; B 330, 491, 616; A 14 II b; MR 1883-1916 exports of, from United States.......................... MR 1883-1916 history of.$\ldots \ldots \ldots \ldots \ldots \ldots \ldots \ldots \ldots \ldots \ldots \ldots \ldots \ldots \ldots \ldots$ A 14 II $b$; MR 1914 imports of, into United States......................... MR 1883-1916 in Alabama.................... W 114; B 32; MR 1883-1916; GF 175; S 2 in Alaska.............................. W 418; B 32; MR 1883, 1884 in Arizona............................ B 32; MR 1883-1916; GF 129 in Arkansas................. W 114, 145; B 32; P 46; MR 1883-1916; GF 154 in Austria-Hungary ..................................... MR 1916 in California.................. W 338; B 32; MR 1883-1916; GF 39, 101 in Colorado......................... B 32; MR 1883-1916; GF 153, 203 in Connecticut...................... W 114, 232; B 32; MR 1883-1916 in Delaware................................ B 32; MR 1883-1916 in District of Columbia.................... W 114; B 32; MR 1896-1914 in England.............................................. 1916 in Florida............................ W 114; B 32; MR 1883-1916 in France.................................................... 1916 in Georgia................. W 114, 160; B 32; MR 1883-1916; GF 187; S 4

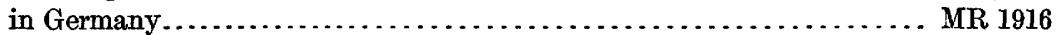

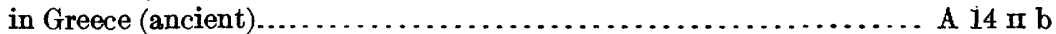
in Idaho................................... B 32; MR 1883-1916 in Illinois........................... W 114; B 32; MR 1883-1916 in Indiana................. W 114; A $18 \mathrm{rv} \mathrm{b;} \mathrm{B} \mathrm{32;} \mathrm{MR} \mathrm{1883-1916;} \mathrm{GF} 84$ in Iowa .............................. W 293; B 32; MR 1883-1916 in Kansas..................... B 32; P 32; MR 1883-1916; GF 148, 159, 206 in Kentucky....................... W 164, 233; B 32; MR 1883-1916 in Louisiana....................... W 114; B 32; P 46; MR 1883-1916 in Maine......................... W 258; B 32; MR 1883-1916; GF 149 in Maryland........................... B 32; MR 1883-1916; GF 152 in Massachusetts......................... W 114; B 32; MR 1883-1916 in Michigan............... W 31, 114; B 32; MR 1883-1916; GF 155, 205 in Minnesota................................ 32; MR 1883-1916 in Mississippi......................... W 114; B 32; MR 1883-1916 in Missouri........................ W 195; B 32; MR 1883-1916; GF 206 in Montana......................... B 32; MR 1883-1916; GF 24, 56 in Nebraska............................... B 32; MR 1905-1916 in Nevada.................................. B 32; MR 1883-1916 in New Hampshire............................ B 32; MR 1883-1916 in New Jersey ........................... W 114; B 32; MR 1883-1916

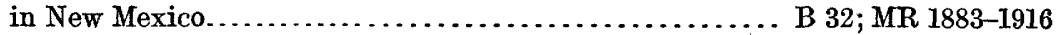
in New York......... W 114, 145; B 32; MR 1883-1916; P 44; GF 92, 169, 190 in North Carolina. . . . . . . . . . . . . . . . . . . . . W 114; B 32; MR 1883-1916 in North Dakota ............................. B 32; MR 1883-1916 in Ohio.................... W 114, 259; B 32; A 18 rv b; MR 1883-1916 in Oklahoma............................... B 32; MR 1883-1916 in Oregon. B $32 ;$ MR 1883-1916

$56122^{\circ}-18-$ WSP $427-8$ 
Mineral waters in Pennsylvania W 114; B 32; MR 1883-1916; GF 92 in Rhode Island. W 114; B 32; MR 1885-1916 in Rome (ancient) A $14 \mathrm{II} \mathrm{b}$ in South Carolina. . . . . . . . . . . . . . . . . . . . . . . . . . . B 32; MR 1883-1916 in South Dakota. . . . . . . . . . . . . . . . . . . . . . . . . . . . MR 1883-1916 in Tennessee............ . . . . . . . . . . . . . . . . W 164; B 32; MR 1883-1916 in Texas.................................. W 66; B 32; MR 1883-1916

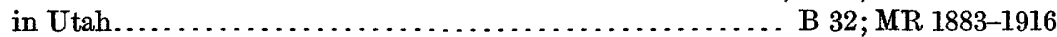
in Vermont............................ W 114; B 32; MR 1883-1916 in Virginia. ............................ B $32 ;$ MR 1883-1916; 8 8 in Washington............................... B 32; MR 1883-1916 in West Virginia . . . . . . . . . . . . . . . . . . . . . W 114; B 32; MR 1883-1916 in Wisconsin . . . . . . . . . . . . . . . . . W 114; B 32; MR 1883-1916; GF 140; S 9 . in Wyoming. ........................ P 53; B 32, $641 \mathrm{i}$; MR 1883-1916

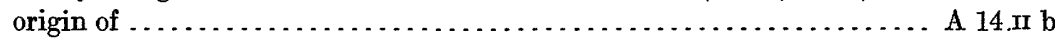
Pliny on ............................................... 14 b production of, in United States. . . . . . . . . . . . . . . . . . . . . MR 1883-1916 radioactivity in, bibliography of . . . . . . . . . . . . . . . . . $1913 \mathrm{Ir} \mathrm{h}$ therapeutic value of. . . . . . . . . . . . . . . . . . . . . . MR $1913 \mathrm{~m} \mathrm{~h}$ statistics of . . . . . . . . . . . . . . . . . . . . . . . . . B $32 ;$ MR 1883-1916 therapeutic properties of. W 195, 259, 338; A $14 \mathrm{II} \mathrm{b}$; MR $1911 \mathrm{II} \mathrm{n}, 1913 \mathrm{In} \mathrm{h}, 1916$ trade in, in the United States...................... A 14 II b; MR 1883-1916 value of, in the United States. . . . . . . . . . . . . . . . . A 14 II b; MR 1883-1916 See also Springs.

Minerals dissolved in ground water. See Analyses and Quality.

Mines, water in..................... W 160, $345 \mathrm{~g} ; \mathrm{B}$ 582; GF 112, 120, 129 water in, fish destroyed by............................... W 273 metals corroded by . . . . . . . . . . . . . . . . . . . . . . . W 273 quality of......... W 273, $345 \mathrm{~g}, 364$; B 330, 491, 529, 606, 616, 625; GF 191 sulphuric acid in ................................. W $273,345 \mathrm{~g}$ Minnekahta limestone, water in, in South Dakota....... W 227; A. 21 Iv b; GF 107

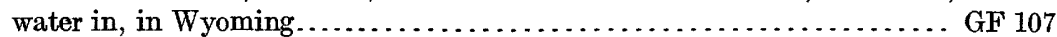

Minnelusa sandstone, water in, in South Dakota.................. W 227, 428;

A $21 \mathrm{Iv} \mathrm{b}$; P 32, 65; GF 107, 127, 128, 164 water in, in Wyoming

A 21 Iv b; P 32, 65; GF 107, 127, 128, 150

Minnesota, areas, all of State........................... W 102, 114 areas, Casselton-Fargo quadrangle. . . . . . . . . . . . . . . . . . . . . . GF 117

Hennepin County. . . . . . . . . . . . . . . . . . . . . . . . . . . B 298

Minneapolis quadrangle................................ GF 201 northwestern ......................................... M 25 Otter Tail County ................................... B 298 Pine County . . . . . . . . . . . . . . . . . . . . . . . . . . . . . . . . B 298 Red River valley.............................. A 11 II c; M 25 St. Paul quadrangle.................................. GF 201

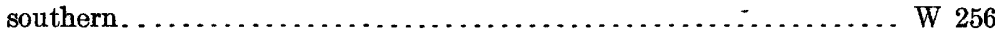
southwestern.......................................... P 32 artesian water in.................. W 114, 256; A 11 in c; M 25; GF 117, 201 bibliography of ground water in................ W 57, 114, 120, 149, 163

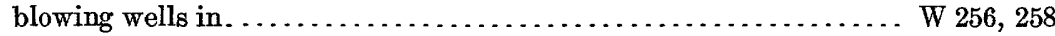
drainage wells in ........................................ W 256

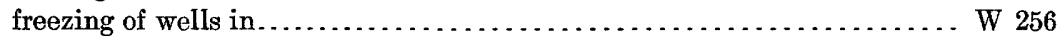
irrigation with artesian water in. ................... A 11 II c; M 25 law relating to ground water in............................ 1.52 
Minnesota, mineral waters in

B 32; MR 1883-1916

public water supplies in W 193, 256 quality of ground water in.... W 102, 193, 256; B 32,330,491, 616; M 25; GF 201 spring records for. . . . . . . . . . . . . well records for. . . . . . . . . . . . . . . . . . . W 57, 149, 256; B 264, 298; M 25 wells in, cost of ................................... A 11 II c

Miocene formations. See under Tertiary and specific formations.

Mississippi, areas, all of State. ......................... W 102, 114, 159 areas, Hancock County. . . . . . . . . . . . . . . . . . . . . B 264, 298

Harrison County . . . . . . . . . . . . . . . . . . . . . . . . . . B 298

Jackson County................................. B 264,298

Jones County ........................................ B 298

Marshall County ...................................... B 298

Newton County........................................ B 298

Panola County. . . . . . . . . . . . . . . . . . . . . . . . . . . . . B 298

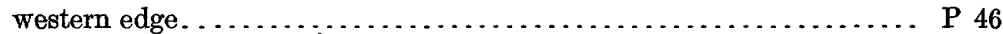

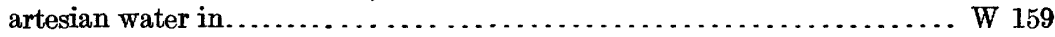

bibliography of ground water in...................... W 114, 120, 163

mineral waters in............................ B 32; MR 1883-1916

quality of ground water in . . . . . . . . . . . . . . . 159,$364 ; \mathrm{B} 32 ; \mathrm{P} 46$

spring records for.................................... B 32

well records for.............................. W 149; B 264, 298

Mississippian formations. See under Carboniferous and specific formations.

Missouri, areas, all of State........................... W 102, 114, 195

areas, Camden County ................................... W 110

Joplin district.............................. W 102, 145; GF 148

Leavenworth quadrangle ................................ 206

Livingston......................................... W 102

Meramec Spring............................ W 99, 131, 173, 209

Ozark region.......................................... W 145

Randolph County................................... B 264

St. Louis quadrangle................................ B 438

Smithville quadrangle................................... GF 206

artesian water in............................ W 195; B 438; GF 148

bibliography of ground water in...................... W 57, 120, 149, 163

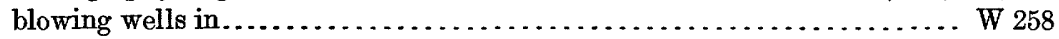

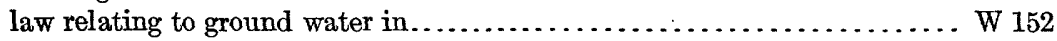
mineral waters in....................... W 195; B 32; MR 1883-1916 public water supplies in ......................... W 195; GF 148

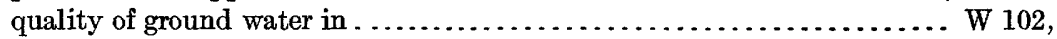

$145,195,364 ; \mathrm{B} 32,330,438,491,606,616$; GF 206 spring records for ....................... W 99, 131, 173, 209; B 32 well records for............................ W 57, 149; B 264, 298 wells in, cost of........................................... W 195

Missouri group, water in, in Iowa.......................... W 293 water in, in Missouri....................................... W 195

Molecular attraction in relation to ground water.................... M 47

Monmouth formation, water in, in Delaware...................... GF 137 water in, in Maryland............................... 137, 182, 204

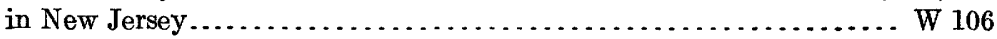

in Pennsylvania........................................... 106

Monroe formation, water in, in Michigan.................... 30,115 
Montana, areas, Aladdin quadrangle............................. GF 128 areas, Butte and vicinity ............................... W $345 \mathrm{~g}$

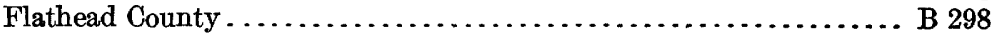

Flathead country ................................... 400 b

Fort Benton quadrangle.................................. GF 55

Great Falls region.................................... W 221

Little Belt Mountain quadrangle ....................... GF 56

Little Bitterroat Valley............................. W $400 \mathrm{~b}$

Musselshell County .................................. B 647

Silver Bow basin ................................. W $345 \mathrm{~g}$

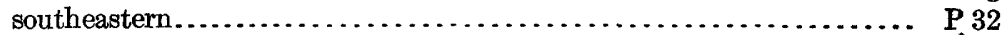

Three Forks quadrangle................................... 24

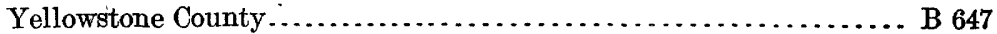

artesian water in................. W 149, 221, $345 \mathrm{~g}, 400$ b; B 647; GF 55, 128

bibliography of ground water in........................ 120,163

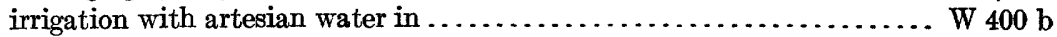

irrigation with ground water in........................ W $345 \mathrm{~g}, 400 \mathrm{~b}$

mineral waters in ..................... B 32; MR 1883-1916; GF 24, 56

public water supplies in................................ W $345 \mathrm{~g}$

quality of ground water in.............W 221, $345 \mathrm{~g}, 364,400 \mathrm{~b} ; \mathrm{B} 32,529,606$

springs in ................................... $130,221,326$; B 32

temperature of ground water in............... W $400 \mathrm{~b} ; \mathrm{B} \mathrm{32;} \mathrm{GF} \mathrm{24,56}$

well records for.............................. 57, 149; B 264, 298

Montana group. See specific formations.

Moore, R. B., Radio activity of thermal waters of Yellowstone National Park. . B 395

Morocco, ground water in, bibliography of......................... W 163

Morrison formation, water in, in Colorado...................... P 52

water in, in South Dakota.................................... 107

in Wyoming................................................ 107

Morrow formation, water in, in Arkansas.................. W 145; GF 122

water in, in Oklahoma .................................. GF 122

"Mortar beds," water in, in Kansas............................... W 273

Mots for lifting water........................................... W 1

Mounds produced by springs.......................... W 277, 320, 423

Mount Selman formation, water in, in Texas.................. W 335, $375 \mathrm{~g}$

Movements of air through porous media........................ A 19 II b

Movements of fluids through rigid media....................... A 19 In b

through wire gauze.................................... 19 II

Movements of ground water, absence of....................... $529 ;$ S 8

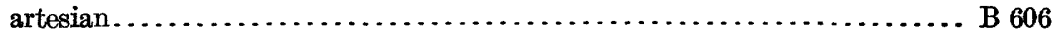

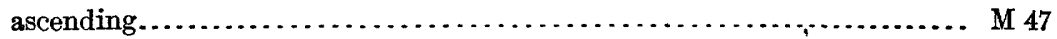

barometric conditions in relation to........................ A 19 m b

bibliography of .................................. 19 II $\mathbf{c}$

decomposition as measure of................................ W 232

deformation of rocks in relation to......................... 19 II b

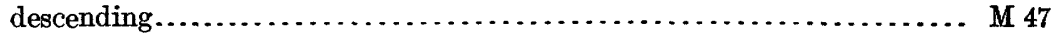

diagrams showing........................... $67,232, \mathrm{M} 47$; B 606

direction of ....................... $67,114,140,184,345 \mathrm{~h} ; \mathrm{A} 19 \mathrm{II}$ b

dynamic changes in relation to............................ 19 II b

faults in relation to...................................... $345 \mathrm{~h}$

fissures in relation to........................... W 160, 232; A 19 п b

fluorescein method of determining.......................... W 160

friction of rocks in relation to....................... A 19 II b; M 47

in Arizona................................... 104, 136, 320, $375 \mathrm{~b}$ 
Movements of ground water in belt of cementation $\ldots \ldots \ldots \ldots \ldots \ldots \ldots \ldots$ M 47

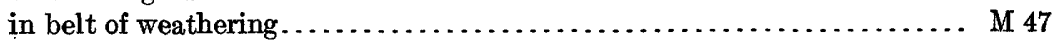

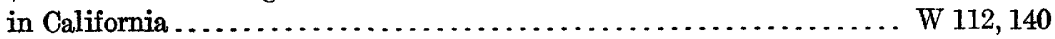

in capillary pores.................................... $19 \mathrm{n} \mathrm{b;} \mathrm{M} 47$

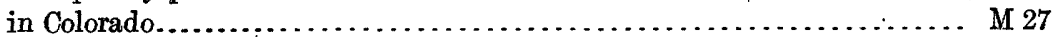

in crystalline rocks............................... 160, 232

in débris-filled valleys................. W 222, 277, 320, $345 \mathrm{~h}, 398,400$ e, 423

in deep zones............................ W 67,$254 ; \mathrm{M} 47 ; \mathrm{B} 529$

in granite.................................... 160,232; GF 158

in Indiana.................................................. 254

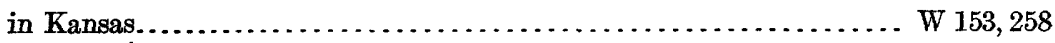

in Nebraska.............................................. 184

in New Mexico .............................. W 141, 158, 275, 343; B 618

in New York................................... W 140; P 44

in North Carolina...................................... S 5

in shallow zones............................ W 254; B 529; S 9

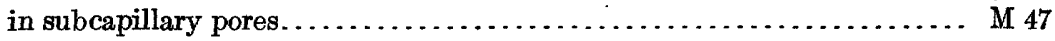

in super-capillary openings. . . . . . . . . . . . . . . . . 47

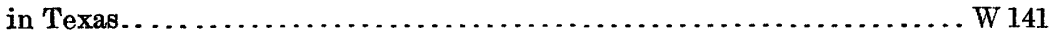

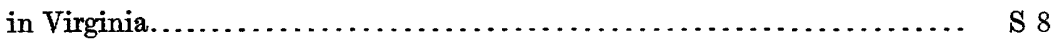

law of ................................ W 67, 140; A 19 II c; M 47

measurements of.................... W 110,112, 140,141, 153, 184, 258; P 44

chlorine method .................................... 67

electrolytic method.................... W 67, 110, 112, 140; P 44

fluorescein method.................................. 160

mechanical analysis method....................... W 104, 136

molecular attraction in relation to............................. 47

precipitation in relation to............................... P 44

pressure in relation to..................... W 67, 140; A 19 u b; M 47 principles of.............. W 67,114, 140,232; A 19 II c, 21 IV c; M 47; B 606

pumping in relation to................................... P 44

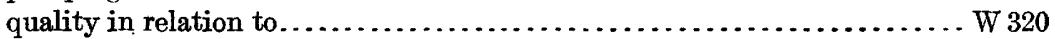

rate of..... W 67, 112, 140, 141, 153, 159, 184, 258; A 16 и f, 19 п b; M 47; P 44; S 8

rock consolidation in relation to........................... 19 m b

salines in Louisiana and Texas in relation to..................... B 282

seepage from reservoirs in relation to ......................... P 44

settling of sediments in relation to.......................... A 19 II b

size of grain in relation to...................... W 67, 104, 136; A 19 II b

temperature in relation to........................... W 140; A 19 In b

thermal.............................................. 19 In $b$

topography in relation to..................................... 67

Municipal water supplies. See Public water supplies.

Murphy, E. C., The windmill.............................. 41,42

Windmills for irrigation $\ldots \ldots \ldots \ldots \ldots \ldots \ldots \ldots \ldots \ldots \ldots \ldots \ldots \ldots \ldots \ldots \ldots \ldots \ldots$, W 8

Myrick formation, water in, in Texas...................W 375 g; GF 64

N.

Nacatoch formation, water in, in Arkansas.................... W 114; P 46

water in, in Louisiana......................................... 114

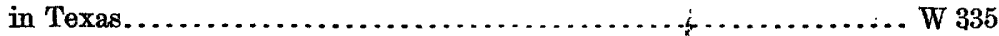

Nanafalia formation, water in, in Alabama..t.................... S 2

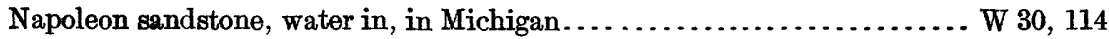

Nashua marl, water in, in Florida............................ W 319 
Natural gas in relation to ground water. . . . . . . . . . . . . . . . 658

Navarro formation, water in, in Texas........................... W 276

Nebraska, areas, all of State............................... P 32

areas, Camp Clark quadrangle.............................. GF 87

Edgemont quadrangle.................................... 108

Elk Point quadrangle.................................. GF 156

Goshen Hole quadrangle............................ W 70

Kearney and vicinity $\ldots \ldots \ldots \ldots \ldots \ldots \ldots \ldots \ldots \ldots \ldots \ldots \ldots \ldots \ldots$ A 21 Iv $a$

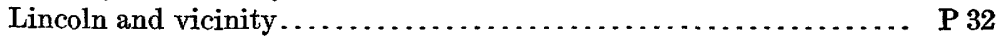

Lodgepole Valley ................................. 425 b

Missouri River valley................................. W 215

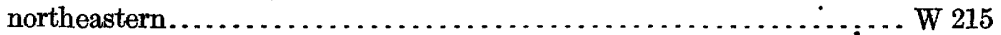

Oelrichs quadrangle........................................... 85

Omaha and vicinity .......................... A 19 IV c; P 17, 32

Patrick quadrangle................................... W 70

Platte River valley.............................. A 21 Iv a

Republican River valley.............................. 216

Scotts Bluff quadrangle................................ GF 88

South Platte Valley...................................... W 184

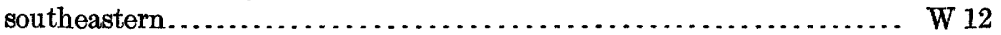

southern tier of counties.............................. W 216

southwestern.......................... A 16 Ir f, 21 Iv c, 22 Iv c

west of $103 \mathrm{~d}$ meridian.......................... A $19 \mathrm{Iv}$ c; P17

artesian water in ............................ 12, 29, 184, 216, $425 \mathrm{~b}$;

A 16 II f, 19 IV c; P 17, 32; GF 85, 87, 88, 108, 156

barometric wells in ..................................... W 29

bibliography of ground water in.................... W 61, 120,149, 163

blowing wells in ............................ W 29, 215, 258; A 16 II f

infiltration tunnels in ..................................... W 184

irrigation with ground water in ................... W 5, 12, 184, 216, $425 \mathrm{~b}$;

A 16 Ir e, 16 Ir f, 19 IV c, 21 IV c, 22 IV c; P 17

law relating to ground water in ............................ 122,152

mineral waters in ........................... B 32; MR 1905-1916

public water supplies in ............................ W 184,425 b

pumping in, cost of ............................... 184, $425 \mathrm{~b}$

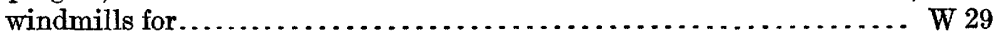

quality of ground water............... W 12, 29, 184, 215, 216; P 32; GF 108

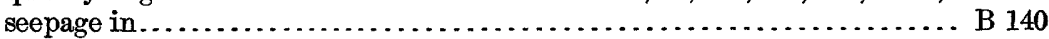

sheet water in ...................................... W $29 ;$ A 16 nf

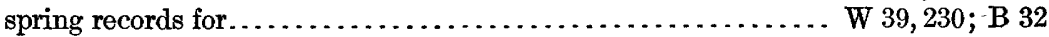

temperature of ground water in ......................... W 215; B 32

underflow measurements in ................................. W 184

well records for........................... W 61, 149; B 131, 264, 298

wells in, construction of.......................... 184, 215, 425 b

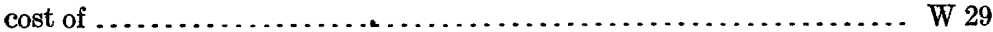

Nellist, J. F., Flowing wells and municipal water supplies in the southern

portion of the southern peninsula of Michigan................... W 182

Nevada, areas, Alkali Spring valley ............................. 423

areas, Big Smoky Valley்................................ W $375 \mathrm{~d}, 423$

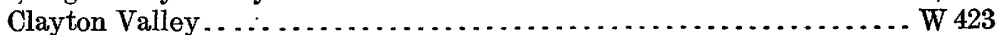

Columbus Marsh............................... P 95 a; B 540 n

Dixie salt marsh ................................... B 540 p

Fourmile Flat. . . . . . . . . . . . . . . . . . . . . . . . . . . B 540 p

Humboldt River valley............................... W 425 d

Las Vegas and vicinity ............................... W 289,365 
Nevada, areas, Railroad Valley ...................... W 365; B 540 p

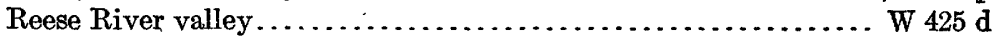

Silver Peak Marsh..................................... B 530 r

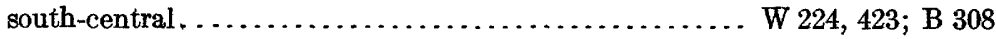

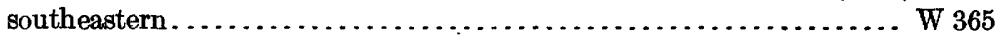

southern................................ W 224, 365; B 308

artesian water in ........................... W 289, 365, $475 \mathrm{~d}, 423$

bibliography of ground water in ...................... W 120, 163

fluctuations of water table in . . . . . . . . . . . . . . . . . . . . W 423

irrigation with ground water in..................... W 365, 375 d, 423

law relating to ground water in......................... W 122, 152

mineral waters in .............................. B 32; MR 1883-1916

public water supplies in . . . . . . . . . . . . . . . . . . W 224, 365, 423

pumping in, cost of ......................................... 423

quality of ground water in .......................... 224 ,

$364,365,375 \mathrm{~d}, 423 ; \mathrm{B} \mathrm{32,529, \textrm {B }} 530 \mathrm{r}, 540 \mathrm{n}, 540 \mathrm{p}, 606$

salt water in................................ W $423 ;$ B 530 r, $540 \mathrm{n}$

springs in.................. W 85, 133, 360, 365, 375 d, 389, 390, 423, B 32

temperature of ground water in .................... W 365, 423; B 32

watering places in............................... W 224, 365; B 308

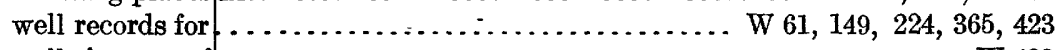

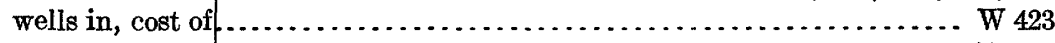

Newark horizon, water in, in Arkansas.......................... W 145

Newark sandstone, water in, in Connecticut............. W 110, 114, 232, 374

water in, in New Jersey........................ W 106; GF 167, 191

in New Yolk ........................................ W 114

in Pennsyl yania........................ W 106, 114; GF 162, 167

Newell, F. H., Proceedings of second conference of engineers of the Reclamation

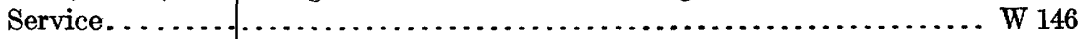

Public lands and their water supply ......................... A 16 II e

Report of division of hydrography for 1893 and $1894 \ldots \ldots \ldots \ldots \ldots \ldots \ldots$ B 131

Report of division of hydrography for $1895 \ldots \ldots \ldots \ldots \ldots \ldots \ldots \ldots \ldots \ldots$ B 140

Stream measurements, $1899 \ldots \ldots \ldots \ldots \ldots \ldots \ldots \ldots \ldots \ldots \ldots \ldots$ A 21 IV a

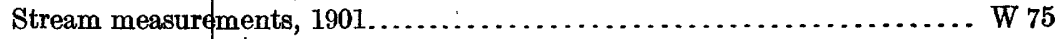

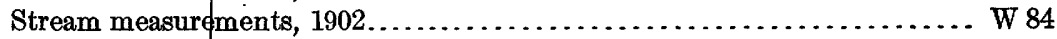

Water supply for irrigation. .......................... A 13 m a

New Hampshire, areas, all of State....................... W 102, 114

areas, Portsmouth-York region. ......................... W 145

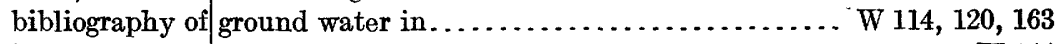

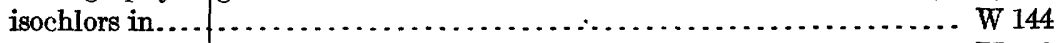

law relating to ground water in . . . . . . . . . . . . . . . . . . . 152

mineral waters in............................ B 32; M R 1883-1916

quality of groupd water in........................... W 102; B 32

spring records fpr............................... W 102; B 32

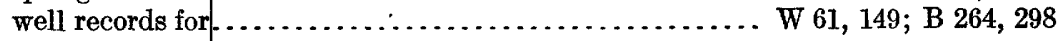

New Jersey, areas, all of State.............................. W 114

areas, Atlantic County . . . . . . . . . . . . . . . . . 264

Coastal Plajn.................................... 138

Dover quadrangle................................... 137

Franklin Furnace quadrangle........................ GF 161

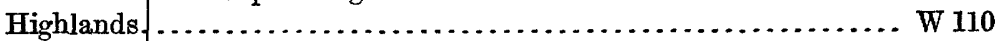

Monmouth County .............................. B 264, 298

Passaic quadrangle....................................... 157 
New Jersey areas, Philadelphia district.................... W 106; GF 162 Raritan quadrangle. . . . . . . . . . . . . . . . . . . . . . . . . . . . 191

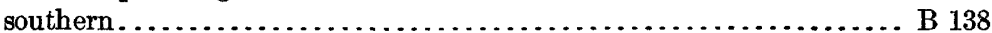
Trenton quadrangle.................................... GF 167 artesian water in........................ W 106; B 138; GF 157, 162 bibliography of ground water in..................W $61,114,120,149,163$ law relating to ground water in .......................... 122, 152 mineral waters in . ...................... W 114; B 32; MR 1883-1916 public water supplies in.......... W 106, 110, 374, 397; B 138; GF 162, 167 quality of ground water in . . . ............... W 258; B 32, 138; GF 191

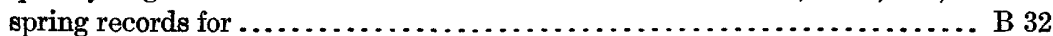

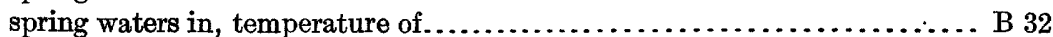
well records for............................... W 61, 149; B 264, 298

New Mexico, areas, Albuquerque district....................... W 188 areas, Animas Valley........................................ 422

Belen district........................................ W 188

central......................................... 260, 275

Deming and vicinity.................... W 345 c; B 618; GF 207

eastern ................................ A $21 \mathrm{rv}$ c, 22 Iv c

Eddy County....................................... B 298

Encino Valley ....................................... W 275

Estancia Valley.................................. 260, 275

Grant County....................................... W 422

Hachita Valley....................................... W 422

Jornada del Muerto . . . . . . . . . . . . . . . . . . . . . . . . . . . W 123, 188

La Mesa district. . . . . . . . . . . . . . . . . . . . . . . . . . . . . . W 188

Luna County............................W 345 c; B 618; GF 207

Mesilla district............................ W 10, 140, 141, 188

Mimbres Valley........................... W 345 c; B 618; GF 207

northwestern.................................. W 380; B 435

Pecos Valley ......................................... 158

Playas Valley . . . . . . . . . . . . . . . . . . . . . . . . . . . . . W 422

Pinos Wells Valley..................................... W 275

Rio Grande valley........................... W 10, 140, 141, 188

Roswell artesian area . . . . . . . . . . . . . . . . . . . . . . . . . W 158

San Luis Valley................................... W 422

San Simon Valley................................... W 425 a

Santa $\mathrm{Fe}$ district..................................... 188

Silver City quadrangle................................. 199

south-central.................................... W 141, 343

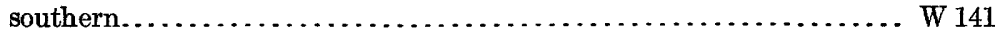

southwestern. . . . . . . . . . . . . . . . . . . . . . . . W 422, 425 a

Torrance County . . . . . . . . . . . . . . . . . . . W 260, 275; B 298

Tularosa Basin ..................................... W 343

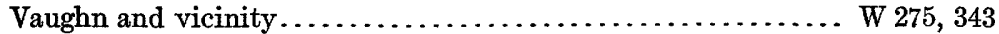

artesian water in $\ldots \ldots \ldots \ldots \ldots \ldots \ldots \ldots$ W 123, 158, 188, 343, 380, 422; GF 199

bibliography of ground water in.................... W 61, 120, 149, 163

infiltration ditches in .................................... 343

irrigation with ground water in ................................. 5 ,

$10,158,188,260,275,343,345$ c, 422,425 a; B $618 ;$ A 16 in e, 21 iv c, 22 Iv c law relating to ground water in . . . . . . . . . . . . . . . . . . . 152 mineral waters in ........................... B 32; MR 1883-1916 public water supplies in .................................. W 343 
New Mexico, pumping in, cost of..................... 10,275, 345 c pumping tests in........................ W 140, 141, 343, 345 c; B 618 quality of ground water in..............W 123, 188, 260, 275, 343, 364, 422, 425 a; B 32, 330, 491, 616, 618; GF 207 quantity of ground water in..................W $141,158,188,275,343$, 345 c. 422 ; B 618 ; GF 207 seepage in ................................................ 358

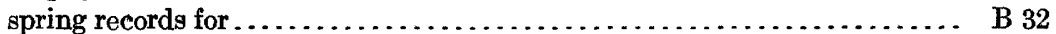
thermal springs in. ................................ B 32; GF 199 well records for . . ............................. W 61, 149; B 264, 298

New Richmond sandstone, water in, in Iowa.................... W 293 water in, in Minnesota..................................... W 256 See, also Lower Magnesian limestone.

New South Wales, bibliography of ground water in ................... W 163

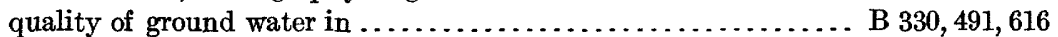

Newsom, J. F., Santa Cruz folio, Cal . ........................... GF 163 New York, areas, all of State................................ W 102, 114 areas, Catatonk quadrangle ......................... W 145; GF 169 Coastal Plain.......................................... B 138

Erie County.......................................... B 298

Gaines quadrangle.................................... GF 92

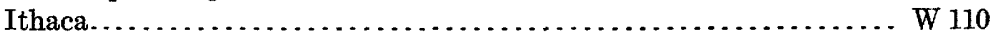

Long Island ........... W 25, 110, 140, 155, 374, 397; P 44; B 138; GF 83

New York City .................................. W 25; GF 83

Niagara quadrangle.................................. GF 190

Passaic quadrangle....................................... GF 157

Taconic quadrangle................................... W 110

Ticonderoga quadrangle.................................. W 110

Tully and vicinity....................................... 145

Watkins Glen quadrangle......................... W 110; GF 169 artesian water in ...................... W 110, 145; P 44; B 138; GF 169 bibliography of ground water in....................W $61,114,120,149,163$ blowing wells in .................................... W 258; P 44 fluctuations of water table in ........................... W 155; P 44

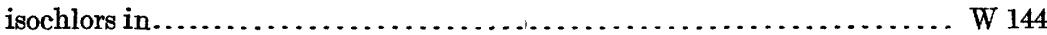
law relating to ground water in ............................... 152 mineral waters in ........... W 114, 145; B 32; MR 1883-1916; GF 92, 169, 190 public water supplies in.... W 25, 110, 155, 374, 397; P 44; B 138; GF 83, 169, 190 quality of ground water in................. W 102, 108, 110,364; P 44; B 32, $330,491,616$; MR 1916; GF 190 radioactive waters in............................... salt water in .......................................... B $530 \mathrm{~b}$ spring records for..................................... $102 ;$ B 32 underflow on Long Island.......................... W 140, 155; P 44 well records for....................... W 61, 102, 149; B 264, 298; P 44

New Zealand, bibliography of ground water in............ W 163; B 330, 491, 616 hot springs in ................................ $9 . \ldots \ldots$ quality of ground water in ............................. B $330,491,616$

Niagara limestone, water in, in Illinois.............. W 114; B 506; GF 81, 200 water in, in Indiana................................ 113, 114, 254

in Iowa......................................... 293 ; 145, 200

in Michigan.......................................... W 30,114

in Ohio................................... 114, 259; A $19 \mathrm{rv}$ b

in Wisconsin.............................. 114; GF 145; S 9 
Nicaragua, bibliography of ground water in.................... W 163

Niobrara formation, water in, in Kansas......................W 273

water in, in Nebraska..................................... 216

in South Dakota.......................... W 227; GF 100, 156

Nitrates in ground water.............. W 256, 338; B 47, 330, 491, 616; MR 1911 II n

Nitroglycerin, use of, in wells.................................. 223

Normal chlorine. See Chlorine and Isochlors.

Normal carbonates in ground water........ W 254, 259, 273, 338, 341, 398; B 330, 491, 616; MR $1911 \mathrm{II}, 1916$

Norristown shale, water in, in New Jersey.......................... W 106

water in, in Pennsylvania............................... 106

North Carolina, areas, all of State.............................. W 114

areas, Coastal Plain................................. 138; $\mathbf{S} 5$

Cowee quadrangle...................................... 110

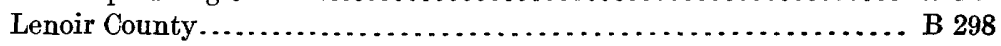

Moore County ..................................... B 298

Mount Mitchell quadrangle.............................. GF 124

New Hanover County ................................. B 298

Norfolk quadrangle..................................... GF 80

Pisgah quadrangle.............................. W 110; GF 147

Roan Mountain quadrangle.............................. GF 151

Wilmington and vicinity................................ 160

artesian water in.............................. B 138; GF 80; S 5

bibliography of ground water in ..............W $61,114,120 ; 149,163$; S 5

law relating to ground water in................................ W 152

mineral waters in .......................... W 114; B 32; MR 1883-1916

public water supplies in............................ B 138; S 5

quality of ground water in ................. W 160, 258, 364; B 32, 138,

$330,491,616$; GF 80,124 ; S 5

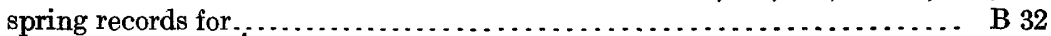

well records for................................... 61,$149 ;$ B 264, 298

North Dakota, areas, all of State............................... A 11 in $\mathrm{c}$

areas, Bismarck quadrangle.................................. GF 181

Casselton-Fargo quadrangle ......................... GF 117

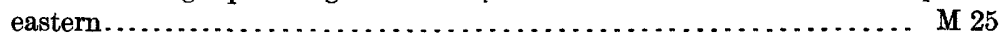

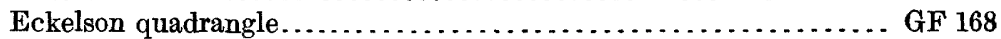

Jamestown quadrangle...................................... GF 168

Morton County........................................ B 575

Red River valley............................... A 11 n c; M 25

southeastern..................................... 17 II g

Standing Rock Indian Reservation...................... B 575

Tower quadrangle.................................. GF 168 artesian water in.............. A 11 II c, 17 II g; M 25; B 575; GF 117, 168 bibliography of ground water in......................W $61,120,149,163$

irrigation with artesian water in................. A 11 II c, 16 II e; M 25

with ground water in........................ A 11 II c, 16 II e; M 25

mineral waters in.......................... B 32; MR 1883-1916

quality of ground water in. . . . . . . . . . . . . A $17 \mathrm{II}$ g; B 32; GF 168; M 25

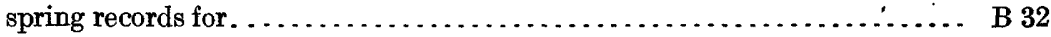

well records for........................................ 61,149

wells in, cost of ....................................... 11 in

Norton, W. H., Underground water resources of Iowa. . . . . . . . . . . . . W 293

Underground waters of Iowa................................ W 114

Water supplies at Waterloo, Iowa............................ W 145

Nussbaum formation, water in, in Colorado.................... GF 135 
O.

Oakville sandstone, water in, in Texas

W $375 \mathrm{~g}$

Occlusion of sea water. See Connate water.

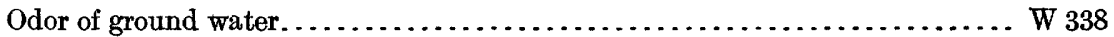

Ogalalla formation, water in, in Colorado......................... P 32

water in, in Kansas........................................ P 32

in Nebraska................... W $425 \mathrm{~b} ; \mathrm{A} 19 \mathrm{IV} \mathrm{c} ; \mathrm{P} 17,32 ;$ GF 87

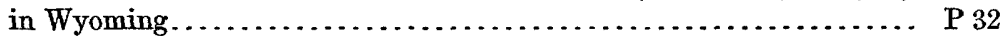

O'Harra, C. C., Aladdin folio, Wyo.-S. Dak.-Mont. . . . . . . . . . . . . . . . GF 128

Belle Fourche folio, S. Dak. ................................. GF 164

Devils Tower folio, Wyo..................................... 150

Ohio, areas, all of State....................... W 114; A 18 Iv b, 19 Iv b

areas, Columbus quadrangle. . . . . . . . . . . . . . . . . . . . . . . . GF 197

Hocking County ........................................ B 298

Kenova quadrangle...................................... GF 184

Miami drainage basin. . . . . . . . . . . . . . . . . 91

Maumee drainage basin .............................. W 91

Muskingum drainage basin. . . . . . . . . . . . . . . . . . . . . . 91

Sandusky drainage basin. .............................. W 91

southwestern. . . . . . . . . . . . . . . . . . . . . . . . . . . . . . W 259

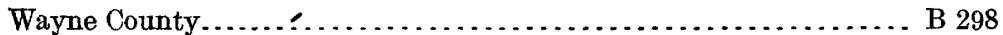

artesian water in................... W 114,259; A 18 Iv b, 19 Iv b; GF 197

bibliography of ground water in ...................W 61,114,120,149, 163

law relating to ground water in ................................ W 152

mineral waters in............... W 114, 259; A $18 \mathrm{Iv} \mathrm{b;} \mathrm{B} \mathrm{32;} \mathrm{MR} \mathrm{1883-1916}$

public water supplies in.................. W 91, 259; A $18 \mathrm{Iv} \mathrm{b,} 19$ Iv b

quality of ground water in.............................. 114,259 ;

A 18 Iv b, 19 Iv b; B 32, 330, 491, 616; GF 184, 197

salt water in............................ A 18 Iv b; B 530 b; GF 184

spring records for $\ldots \ldots \ldots \ldots \ldots \ldots \ldots \ldots \ldots \ldots \ldots \ldots \ldots \ldots \ldots \ldots \ldots, \quad$ B 32

well records for.............. W 61, 91, 149, 259; A 18 Iv b, 19 Iv b; B 264, 298

Ohio shale, water in, in Kentucky. . . . . . . . . . . . . . . . . . . . . . . . W 233 water in, in Ohio.................................... 19 Iv b

Oil, ground water in relation to............................ $653,658,661 \mathrm{~d}$ quality of ground water in relation to ..................... $653,661 \mathrm{~d}$

Oil wells, pollution of ground water by . . . . . . . . . . . . . . . W 113, 257 water in . . . . . . . . . . . . . . . $282,653,658,661 \mathrm{~d}$

Oklahoma, areas, Black Prairie.............................. A 21 vII areas, central............................. W 148; A 21 Iv c, 22 Iv c

Cherokee Nation....................................... B 298

Chickasaw Nation. . . . . . . . . . . . . . . . . . . . . . . . . . . . . B 298

Creek Nation........................................ B 298

Cushing oil and gas field. ............................... B 658

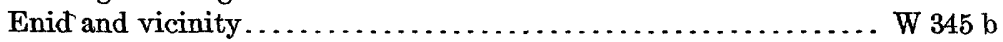

Foraker quadrangle.................................. B 641 b

Grand Prairie..................................... A 21 vil

Muscogee quadrangle.................................. GF 132

north-central ..................................... B $691 \mathrm{c}$

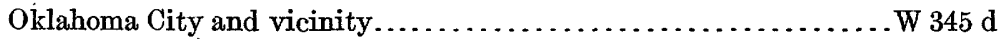

Osage County....................................... B 641 b

Pawhuska quadrangle................................... B $691 \mathrm{c}$

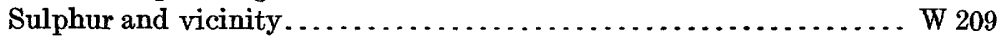

Tahlequah quadrangle.................................. GF 122

western............................. W 148; A 21 rv c, 22 Iv c

Winslow quadrangle................................ GF 154 
Oklahoma, artesian water in............................... 148 bibliography of ground water in ........................ 120, 163 irrigation with ground water in..... W 5, 148, 345 b, d; A 16 II e, 21 IV c,22 IV c law relating to ground water in .......................... 122,152 mineral waters in. ......................... B $32 ;$ MR 1883-1916 pumping in, cost of................................ $345 \mathrm{~b}, \mathrm{~d}$ quality of ground water in................. W 148, $345 \mathrm{~b}, \mathrm{~d}, 364 ; \mathrm{B} 606$ salt water in .................................... 148 ; GF 122 spring records for............................ $99,209,247 ; \mathrm{B} 32$ well records for. ........................... $57,61,149 ; \mathrm{B} 264,298$

Oligocene formations. See under Tertiary; also see specific formations.

Oneota limestone, water in, in Towa......................... W 293 water in, in Minnesota................................. W 256

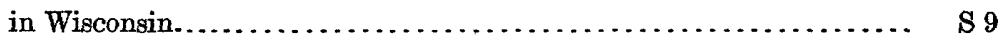

See also Lower Magnesian limestone.

Onion Greek marl, water in, in Texas. ........................ 18 In b

Onondaga limestone, water in, in Ohio.................. W 114; A 19 rv b

Opeche formation, water in, in South Dakota................... GF 107

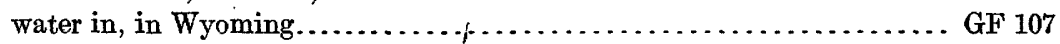

Ordovician formations, water in, in Alabama............... 114; GF 175 water in, in Arkansas.................................. 114, 145

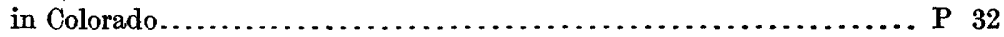

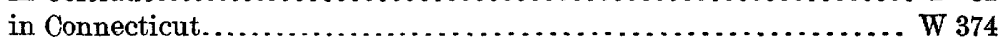

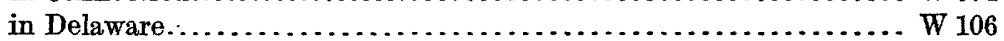

in Georgia............................................... 114

in Illinois...... W 114; A 17 II h; M 38; B 438, 506; GF 81, 145, 185, 188, 200

in Indiana......................... $113,114,254 ; A 18$ Iv b.

in Iowa............................ W 114, 145, 293; GF 145, 200

in Kansas........................................... 148, 206

in Kentucky.................................... W 114, 233

in Maine............................................. GF 158

in Maryland ........................................ 179

in Michigan ...................................... 30,114

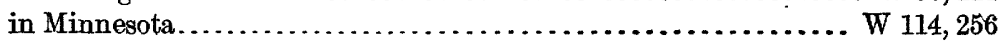

in Missouri..................... W 114, 145, 195; B 438; GF 148

in Nebraska............................................. P 32

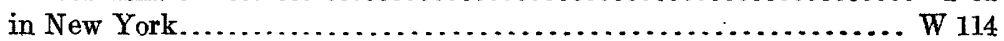

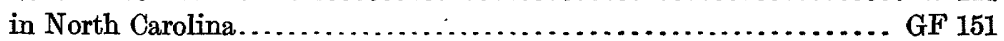

in Ohio........................ W 114, 259; A 18 Iv b, 19 Iv b

in Pennsylvania................. W 106, 110, 114; GF 162, 170, 179

in South Dakota..................................... $\mathbf{P} 32$

in Tennessee................................... 114; GF 151

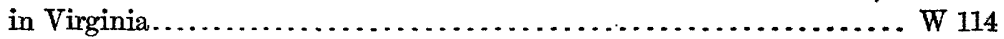

in West Virginia............................ W 110, 114; GF 179

in Wisconsin............................ 114, 145; GF 140, 145; S 9

in Wyoming .............................................. P 32

See also specific formations.

Ore deposits, ground water in relation to......................... 160 ,

See also Water table.

$273,345 \mathrm{~g} ; \mathrm{M} \mathrm{47} ; \mathrm{B} 529,582,606 ;$ GF $112,120,129$ 
Oregon, areas, Abert Lake basin ................................ W 220

areas, Alkali Lake basin.................................. W 220

Alvord basin........................................... 231

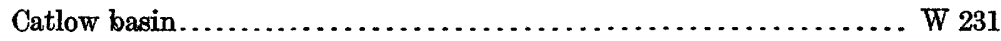

central.............................................. B 252

Chewaucan Marsh basin................................. W 220

Christmas Lake basin ................................... W 220

Goose Lake basin ...................................... W 220

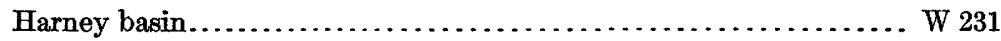

Malheur River basin ................................... W 231

Nampa quadrangle.................................... GF 103

Silver Lake basin.................................. W 220

south central............................................ W 220

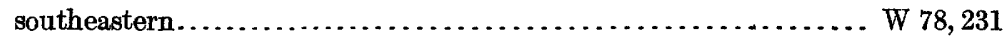

Summer Lake basin .................................. W 220

Warner Valley .......................................... W 220

Whitehorse basin..................................... W 231

artesian water in ..................... W 78, 220, 231; B 252; GF 103

bibliography of ground water in ............................. W 120,163

blowing wells in ......................................... W 258 irrigation with ground water in.............. W 220, 231; A 16 II e; GF 103 law relating to ground water in........................... W $122 ; 152$ mineral waters in .............................. B 32; MR 1883-1916 quality of ground water in ............................ W 220; B 32 spring records for....... W 214, 250, 251, 252, 271, 310, 360, 370, 390, 391, 394; B 32 temperature of ground water in .................... W 220, 231; B 32, 252 well records for....................................... W 61,149 wells in, construction of ............................. W 78, 220, 231

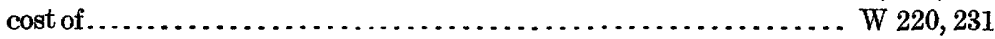

Origin of ground water........................ W 9, 67, 114, 142, 155, 158, $219,222,233,254,294,343,345$ h, 375 a, b, d, 398, 399, 400 e, 423; B 319, 529, 618; A 14 II b, 16 II f, 17 ri g, 22 Iv c; $\mathrm{P} 44,90 \mathrm{~h} ; \mathrm{S} 7$ ancient writers on ........................................ A 14 II b Descartes on ........................................... A 14 пा b erroneous ideas in regard to............................. W 254, 278 from deep fissures...................................... $400 \mathrm{~b}$

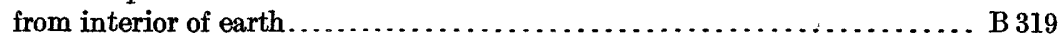

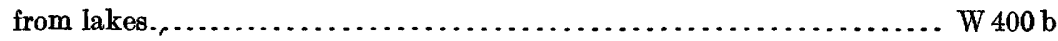
from ocean ............................................ B 319

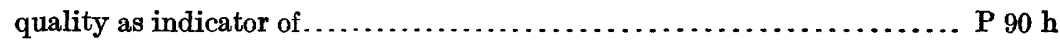
See also Absorption and Quantity.

Origin of mineral springs ............................. W 338; A 14 II b Orinda formation, water in, in California........................ GF 193 Orton, Edward, Rock waters of Ohio........................... A 19 Iv b

Osage group, water in, in Illinois . ............................. B 438

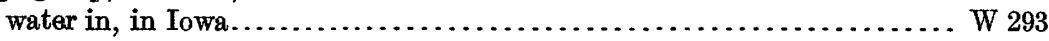

in Missouri............................................. B 438

Outwash deposits. See Glacial drift............................... W 110

Oxidation of ores, sulphates produced by ..................... W $273,345 \mathrm{~g}$

Oxidation of rocks, ground-water levels in relation to........... P 94; B 529, 625

Oxidation produced by ground water.............................. M 47 
P.

Packers for wells. See Well construction.

Paecottahs for lifting water.

Pahasapa limestone, water in, in South Dakota...... W 227; P 32; GF 107, 128, 164 water in, in Wyoming...................... P 32; GF 107, 128, 150

Paige, Sidney, Llano-Burnet folio, Tex........................ GF 183

Silver City folio, N. Mex.................................. 199

Palache, Charles, Franklin Furnace folio, N. J..................... GF 161

Paleozoic formations. See specific formations and rock systems.

Palmer, Chase, Deep well at Charleston, S. C...................... P $90 \mathrm{~h}$ Geochemical interpretation of water analyses...................... B 479

Quality of the waters in the Blue Grass region, Ky.................. W 233

Paluxy sand, water in, in Texas................................ A 21 vII

Pamunkey formation, water in, in Atlantic Coastal Plain................. B 138 water in, in Maryland.......................... B 138; GF 13, 23, 204

in Virginia......................... W 114; B 138; GF 13, 23; S 8

Panola formation, water in, in Kentucky......................... W 233

Paper manufacturing, quality of water for .................. 254, 341, 398

Parker, H. N:, Quality of some waters of the Coastal Plain of North Carolina.. S 4

Quality of water in Kansas.................................... W 273

Parkman sandstone, water in, in Wyoming............... P 51; GF 141, 142

Parks, E. M., Lignite field of northwestern South Dakota.................... B 627

Parma sandstone, water in, in Michigan........................ W 30,114

Pascagoula formation, water in, in Mississippi........................ W 159

Patapsco formation, water in, in Maryland......................... GF 204

Patuxent formation, water in, in Maryland........................ GF 204

water in, in North Carolina................................... $\mathrm{s} 5$

Payette formation, water in, in Idaho............................. GF 45

Peale, A. C., Bibliography of mineral waters........................... 1905

Mineral springs of the United States.......................... B 32

Natural mineral waters of the United States.................... A 14 II b

Production of mineral waters................................. 1883-1900

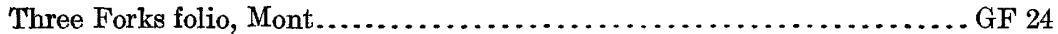

Peay sand, water in, in Wyoming.................................. 6211,656

Peedee sand, water in, in North Carolina............................ S 5

Pendleton sandstone, water in, in Indiana......................... W 254

Pennsylvania, areas, Accident quadrangle........................... GF 160

areas, all of State............................................... 114

Amity quadrangle............................ B 300; GF 144

Barnesboro quadrangle....................... W 110; B 531 d; GF 189

central............................................. B $531 \mathrm{~d}$

Chambersburg quadrangle......................... W 110; GF 170

Curwensville quadrangle.......................... W 110; B $531 \mathrm{~d}$

Ebensburg quadrangle........................... W 110; GF 133

Elders Ridge quadrangle......................... W 110; GF 123

Gaines quadrangle........................................ 92

Grantsville quadrangle................................. GF 160

Hancock quadrangle.............................. W 145; GF 179

Houtzdale quadrangle............................... B $531 \mathrm{~d}$

Indiana quadrangle..................................... GF 102

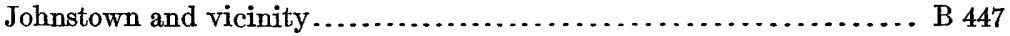

Johnstown quadrangle.............................. GF 174

Mercersburg quadrangle.......................... W 110; GF 170 
Pennsylvania, areas, Patton quadrangle................ W 110; B 531 d; GF 189 Pawpaw quadrangle................................ W 145; GF 179 Philadelphia district......................... W 106; B 138; GF 162 Punxsutawney quadrangle............................... B $531 \mathrm{~d}$

Rogersville quadrangle................................ GF 146

Trenton quadrangle..................................... GF 167

Warren County ......................................... B 298

Warren quadrangle......................................... GF 172

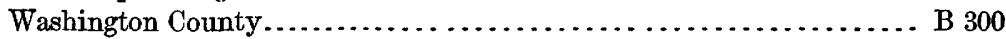

Waynesburg quadrangle....................... W 110; GF 121 artesian water in........... W 106; B 138, $531 \mathrm{~d}$; GF 102, 121, 133, 160, 179, 189 bibliography of ground water in...................W 61, 114, 120, 149, 163 law relating to ground water in ............................... W 152 mineral waters in.................. W 114; B 32; GF 92; MR 1883-1916 public water supplies in.... W 106, 110; B 447; GF 121, 123, 162; GF 167, 170, 189 quality of ground water in.......... W 108, 110, 364; B 32, 300, 447; GF 144, 179 spring records for....................................... B 32 well records for.............................. W 61, 149; B 264, 298

Pennsylvanian formations. See under Carboniferous and specific formations.

Perched ground water.....................W 164, 233, 258, 320, 335; P 44, 46; S 8

flowing wells from ....................................... W 320

Percolation experiments.................................... A $19 \mathrm{~m} \mathrm{~b}$

Percolation. See Movements of ground water.

Perforation of casings................................. W 110, 140, 257

Perkins, G. F., Underground waters of Vermont.......................... W 114

Well and spring records for Vermont............................ W 102

Permeability of rocks........................................ 67,114

Permian formations. See under Carboniferous and specific formations.

Perry, T. O., Experiments with windmills................................ W 20

Persalinity of ground water...................................... W 338

Persia, bibliography of ground water in............................. W 163

Persian wheels for lifting water................................... W 1

Peru, bibliography of ground water in.............................. W 163

Petroleum. See Oil.

Phalen, W. C., Ellijay folio, Ga.-N. C.-Tenn........................ GF 187

Johnstown folio, Pa..................................... GF 174

Kenova folio, Ky.-W. Va.-Ohio.............................. GF 184

Mineral resources of Johnstown, Pa........................... B 447

Potash in bitterns of eastern United States...................... B $530 \mathrm{~b}$

Philippine Islands, bibliography of ground water in................... W 163

quality of ground water in.......................... B $330,491,616$

Phosphates in ground water.............. W 338; B 47, 330,491, 616; MR 1911 In n

Photographs. See Illustrations.

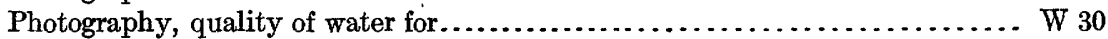

Phreatic water, definition of.................................. A 14 II a

Physiological effects of water............................... MR $1911 \mathrm{n}$

Pierce, C. H., Water resources of Hawaii, 1909-1911.................... W 318

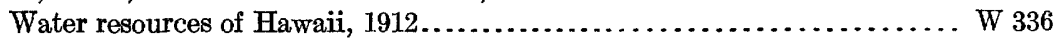

Pierre shale, water in, in Kansas............................... W 273

water in, in Nebraska..................................... W 216

in South Dakota.......................... W 227; GF 113, 114, 165

Piney sandstone, water in, in Wyoming........................... P 51

Pishel, M. A., Geology of the Standing Rock and Cheyenne River Indian reservations, N. Dak.-S. Dak................................... B 575 


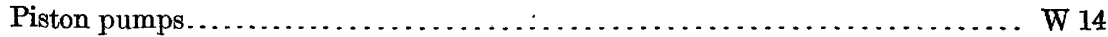

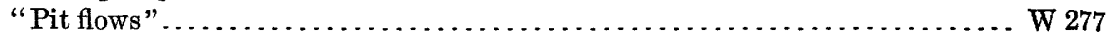

Pitkin limestone, water in, in Arkansas..................... W 145; GF 154

water in, in Oklahoma....................................... 154

Pittsburgh sandstone, water in, in Pennsylvania.................... GF 123

Plains marl, water in, in Colorado.............................. A 16 nf

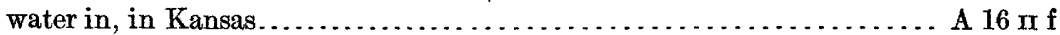

in Nebraska................................... A 16 uf

Plastic formations, water levels in relation to ...................... W 155

Plants. See Transpiration and Vegetation.

Plates. See Illustrations.

Platteville limestone, water in, in Iowa ................... W 293; GF 145 water in, in Minnesota....................................... 256 water in, in Wisconsin................................. 145 ; 9 See also Trenton limestone.

Plattsburg limestone, water in, in Kansas......................... GF 206 water in, in Missouri ..................................... GF 206

Pleasanton shale, water in. in Missouri ......................... W 195

Pleistocene deposits. See Glacial drift, Quaternary deposits, and Valley fill.

Pliny on mineral waters.................................... A $14 \mathrm{nI}$ b

Pliocene formations. See under Tertiary and specific formations.

Plunger pumps............................................... W 14

Plutonic waters. See Juvenile waters and Magmatic waters.

Pocono formation, water in, in Pennsylvania...................... B 300

Point Pleasant formation, water in, in Ohio....................... W 259

Poison Canyon formation, water in, in Colorado.................... GF 71

Pollution of artesian water.................................... S 3

Pollution of cisterns........................................... 255

Pollution of ground water................ W 110, 145, 160, 255, 293; A 14 In a; S 9 ${ }^{\bullet}$ bacteria in relation to................W 193, 256, 259, 335, 341, 398, 399; $\mathrm{S} \mathrm{4,} 11$

by drainage wells........................................ 258

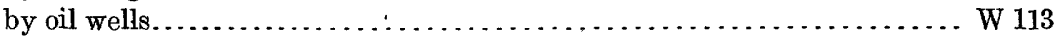

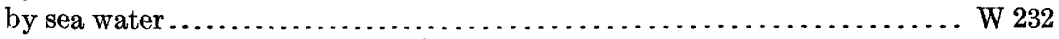

by sewage................................. $232,255,293 ; \mathrm{s} \mathrm{4,11}$

by $\operatorname{sink}$ holes....................................... 255,258

chlorine as an indicator of ................ W 114, 144,223, 232, 256,398; S 4

defective casings as cause of ........................ W 257, 259

fluorescein as an indicator of ........................... W $160 ;$ S 11

history of United States in relation to ...................... A $14 \mathbf{u ~ a ~}$

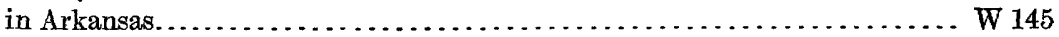

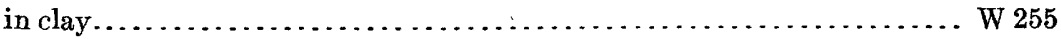

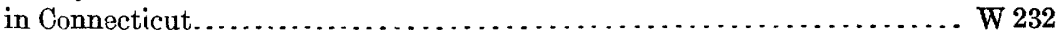

in Georgia.......................................... 41

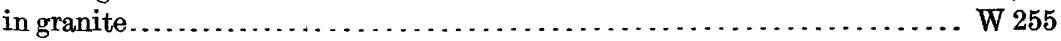

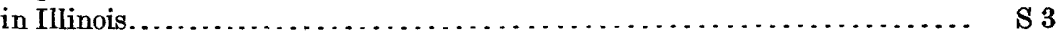

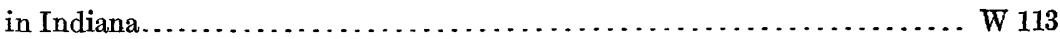

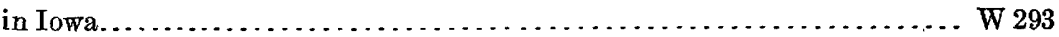

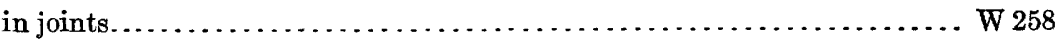

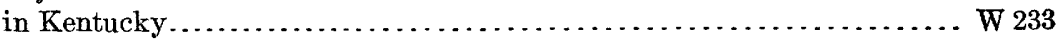

in limestone....................................... 255, 258; S 3

in Maine . . . . . . . . . . . . . . . . . . . . . . . . . . . 223

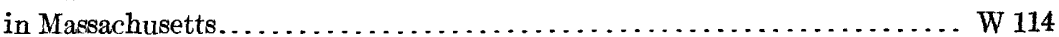

in Michigan ................................... 30,31 
Pollution of ground water in Minnesota..................... 193, 256

in Mississippi............................................. 159

in Rhode Island . . . . . . . . . . . . . . . . . . . . . . . . . . . . . . . . . W 114

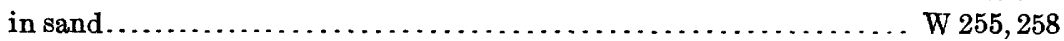

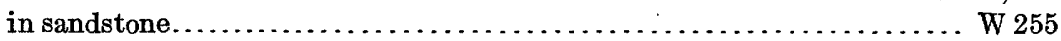

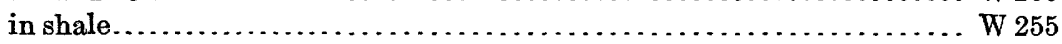

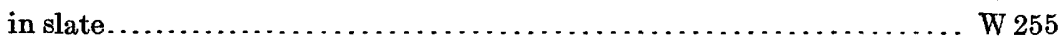

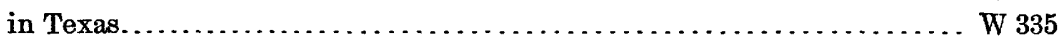

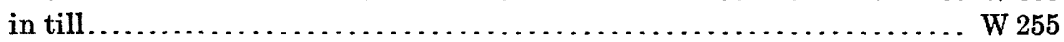

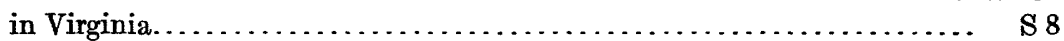

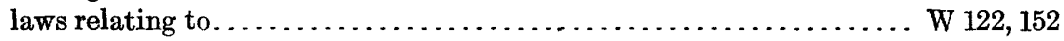

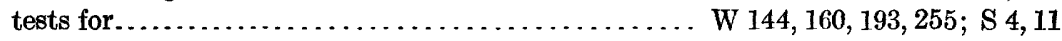

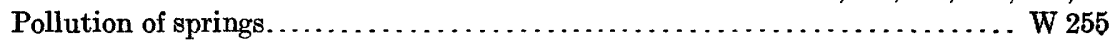
See also Púrification of ground water.

Pollution of water, definition of term .......................... W 315

Pollution of wells................. W 110, 145, 160, 223, 232, 255, 257, 293; S 4, 11 methods of preventing ............................... 160,255

"Ponded" ground water................................ 8

Pool springs.................................... W 221, 277, 365, 423

Porosity, methods of determining...................... W 400 e; A 19 m b of crystalline rocks................................. W 160, 232 of gravels.......................................... 104, 136, 140 of rocks................................ $67,114,160 ;$ A 19 пा b of sand .......................... 6 67, 140, 294, 400 e; A 19 Ir b of soils........................................ $67 ; 19 \mathrm{II}$ b; S 8 of valley fill.......................... $222,294,345 \mathrm{~h}, 398,400 \mathrm{e}$ of water-bearing materials . . . . . . . . . . . . . . $114,136,140,160,232,294,400$ e; $\AA 17$ II f, 19 II b; M 47; S 2 of wet material above the water table.................. A 17 II f, 19 in b practical............................................. 104,136

Port Hudson formation, water in, in Arkansas.................. 46 water in, in Louisiana.................................... 46

Porters Creek formation, water in, in Missouri................... W 195

Porto Rico, bibliography of ground water in..................... W 120

.Potability of ground water, mineral content in relation to...... . W 341, 343, 398, 399

Potassium in ground water .......... W 338, 343; B $530 \mathrm{~b}, \mathrm{r}, 540 \mathrm{n}, \mathrm{p}$; MR 1911 II n

Potomac group, water in, in Atlantic Coastal Plain ................. B 138 water in, in Delaware.............................. 138; GF 137 in District of Columbia.......................... 138; GF 70 in Georgia...................................... W 341; S 4 in Maryland........................ B 138; GF 13, 70, 137, 182, 204 in North Carolina.................................... S 5 in Virginia........................... 114; B 138; GF 13, 70; $\mathrm{S} 8$

Potsdam sandstone, water in............................... 67 water in, in Illinois.................. W 144; A 17 II h; M 38; GF 81, 200 in Iowa....................................... 293 GF 145, 200 in Wisconsin........................ 114, 145; GF 140, 145; 99 See also Cambrian sandstone, Dresbach sandstone, and Jordan sandstone.

Pottsville formation, water in, in Illinois.......................... GF 185 water in, in Pennsylvania........................................ 300

Powell, J. W., Artesian irrigation on Great Plains.................. A 11 II c $56122^{\circ}-18-$ wsP $427-9$ 


Pump rods, stresses of...................................... 14

Pumping, cost of. ........................................ 13 m b

cost of, in Arizona. . . . . . . . . . . . . . . . .

in California................ W 116, 137, 138, 139, 219, 222, 375 a, 398

in Colorado...................................... W 9

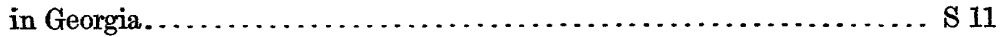

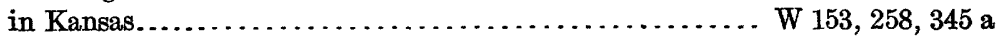

in Louisiana............................................. 101

in Nebraska................................... $184,425 \mathrm{~b}$

in Nevada................................... W $375 \mathrm{~d}, 423$

in New Mexico..................... W 10, 140, 141, 260, 275, $345 \mathrm{c}$

in Oklahoma.......................................... $345 \mathrm{~b}, \mathrm{~d}$

in Texas......................................... 13, 140, 141

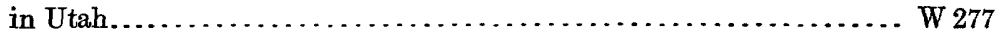

in Washington. . . . . . . . . . . . . . . . . . . . . $425 \mathrm{e}$

in Wyoming. ....................................... $425 \mathrm{~b}$

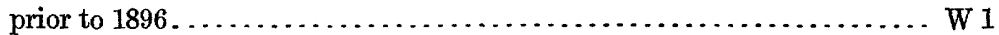

size of plant in relation to . . ......................W $375 \mathrm{a}, 398$

fluctuations of water table in relation to........ W 142, 219, $345 \mathrm{~h}, 155 ; \mathrm{P} 44$

for irrigation................................ 20,$146 ; A 13 \mathrm{mr} b$

in Arizona........................... W 104, 136, 320, $375 \mathrm{~b}, 425$ a

in Arkansas. . . . . . . . . . . . . . . . . . . . . . . . . . . . . . . W 399

in California............... W $17,45,58,59,60,89,137,138,139,142$

$146,181,219,222,278,345$ h, 375 a, 398

in Colorado .......................................... W 9

in Hawaii.................................... 318,$336 ; \mathbf{S} 12$

in Idaho.......................................... 103

in Kansas........................... 8, 153, 258, $345 \mathrm{a}, \mathrm{b}$

in Louisiana. ...................................... 101

in Nebraska............................. $12,184,425 \mathrm{~b}$

in Nevada.................................W 365, $375 \mathrm{~d}, 423$

in New Mexico...........W 10, 140, 141, 188, 260, 275, 343, $345 \mathrm{c} ; \mathrm{B} 618$

in Oklahoma..................................... $345 \mathrm{~b}, \mathrm{~d}$

in Oregon............................. W 220, 231; GF 103

in Texas..................................... 13, 140, 141

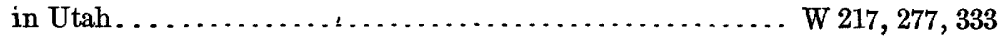

in Washington. . . . . . . . . . . . . . . . . . 425 e

in Wyoming. . . . . . . . . . . . . . . . . . . . $425 \mathrm{~b}$

of rice....................................... 101,399

movements of ground water in relation to..................... P 44

porosity estimates based on .............................. $400 \mathrm{e}$

Pumping plants. See Pumps and other lifting devices.

Pumping tests ............. W 140, 141, 153, 258, 320, 333, 345 c, 398, 425 b; B 618

Pumps and other lifting devices, air lifts..................... W 256, 343

antiquated............................................ $W_{1}$

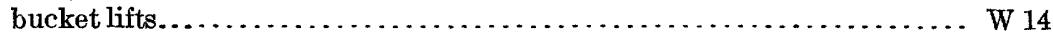

doons. . . . . . . . . . . . . . . . . . . . . . 1

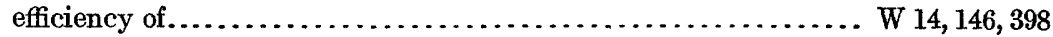

hydraulic rams............................. $1,254,256,258$; $\mathrm{S} 8$

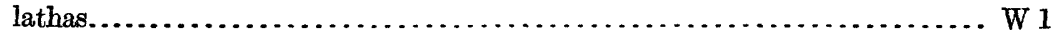

mechanical elevators......................................... 1,14

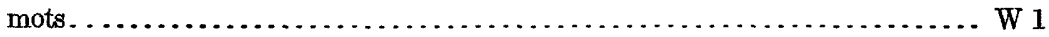

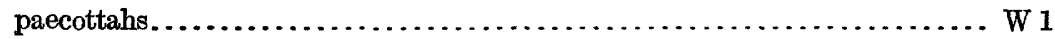


Pumps and other lifting devices, Persian wheels $\ldots \ldots \ldots \ldots \ldots \ldots \ldots \ldots \ldots \ldots$ W 1

Seaman bucket lift......................................... W 14

shadoofs.....................................................

siphon elevators................................................. 1

siphons.................................... W 254, 320, 374, 397

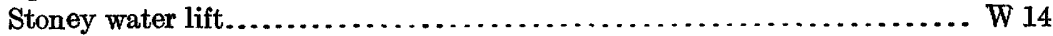

swinging wheels....................................... 1

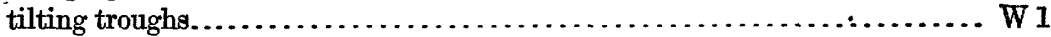

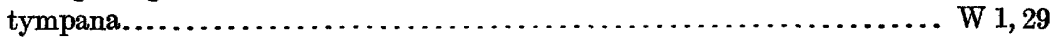

unusual types of .............................. W 1,29; A 13 IIr b

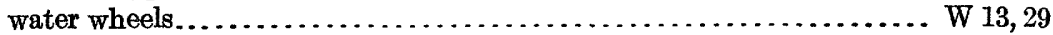

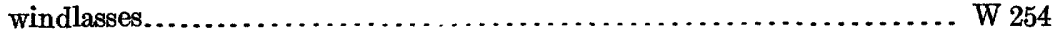

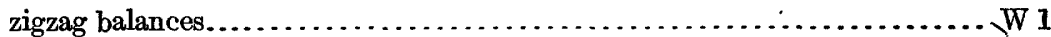

Pumps, centrifugal............................. W 1, 184, 320, 375 a, 398

clack valves in ................................................ 14

cylinder.................................................... 14

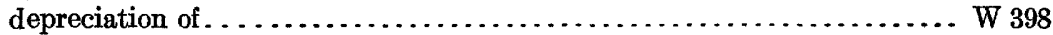

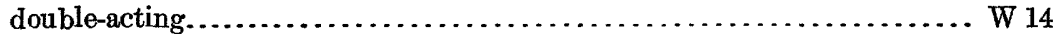

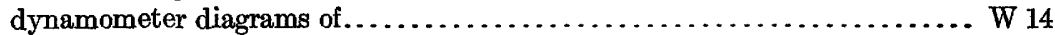

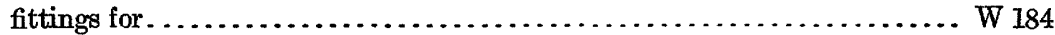

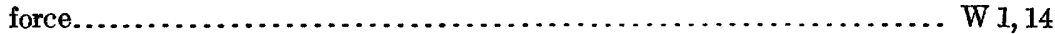

independent of well casings, advantages of................. W 256, 293

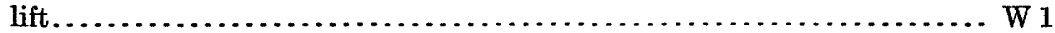

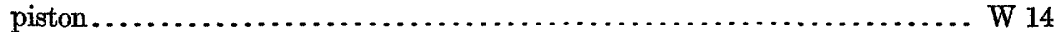

plunger. . . . . . . . . . . . . . . . . . . . . 1,14

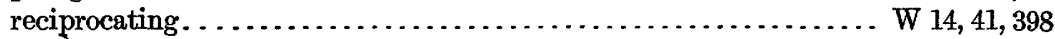

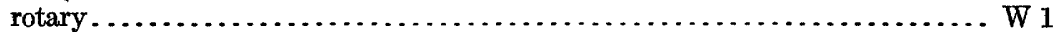

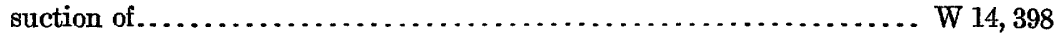

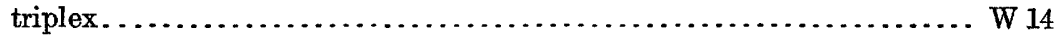

valves in.................................................. 14

Purdue, A. H., Underground waters of northern Arkansas............... W 114

Water resources near contact between Paleozoic and Mississippi embayments, northern Arkansas..................................... W 145

Water resources of the Winslow quadrangle, Ark................. W 145

Well and spring records for Arkansas........................... W 102

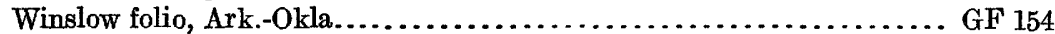

Purgatoire formation, water in, in Colorado . . ..................... GF 186

Purification of ground water, natural......................... W 255, 258

Purification of water by cold-water softening........ W 254, 259, 293, 341, 398, 399

by distillation........................................ W 254

by feed-water heating. . . . . . . . . . . . . . . . W 254, 259, 293, 341, 398, 399

by filters in cisterns.................................. 14 II a

by lime and soda-ash softening................................... 315

by mechanical filtration................................. 254,259

by natural filtration . . . . . . . . . . . . . . . . . . . . . . . . W 255, 258

by slow sand filtration.....................W 254, 259, 315, 341, 398, 399

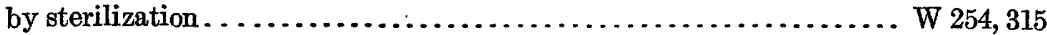

filtration tests for.............................................. 44

history of, in the United States. . . . . . . . . . . . . . . . . . . . . 315

methods of . . . .................... W 254, 259, 293, 315, 341, 398, 399; S 9

Pynchon, W. H. C., Drilled wells of the Triassic area of the Connecticut Valley. W 110 


Quality of ground water, regarding fluorides. B 47; MR 1911 in $n$ gases in solution.................................... M 47 gold............................................... P 94 gypsum...........................W 273, 317, 320,343, 398; S 1 hardness............... W 151, 232, 254, 259, 273, 320, 338, 341, 343, 398, 399 hydrogen sulphide................... W 254, 259, 338, 341, 398, 399; B 282, 398, 447; MR 1911 in n; GF 174; S 2 hydroxides. MR 1911 ir $\mathrm{n}$ iodides. W 233, 338; B 47; MR 1911 in $\mathrm{n}$ iron W 254, 256, 259, 273, 338, 341, 398, 399; MR 1911 II n, 1916; GF 206; S 2 iron and fixed nitrogen................................ W 256 lead. W 254, 399; B 47, 606 lithium W 160, 259,338; B 47; MR 1911 II n magnesium . . ............ W 254, 259, 273, 338, 341,343, 398; MR 1911 II $\mathrm{n}$ manganese ........................... W 338; B 47; MR 1911 in $n$ neutrality .................................. B 479, 606 nitrates............... W 256, 338; B 47, 330, 491, 616; MR 1911 Ir $\mathrm{n}$ odor............................................... W 338 organic matter.......................... W 193, 254, 259, 341, 398 permanent hardness................................. W 338

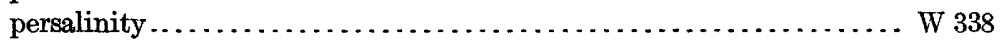
phosphates.................. W 338; B 47, 330,491, 616; MR 1911 in n potassium .............. W 338, 343; B 530 b, r, 540 n, p; MR 1911 in n radioactivity ....................... W 338; B 395; MR 1913 in n rubidium . . . . . . . . . . . . . . . . 47 salinity . . . . . . . . . . . . . . . . . . . . . . . . $338 ; 9,606$ salt, in Alabama................................... 114; S 2 in Antigua . . . . . . . . . . . . . . . . . 10 in Arizona.................... W 104, 136, 320, 375 b; GF 129 in California........................... W 58; B $540 \mathrm{n}, 653$ in Colorado...................................... B $531 \mathrm{c}$ in Delaware........................................ W 258

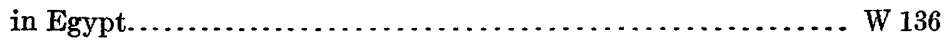

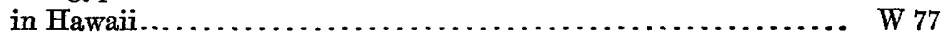

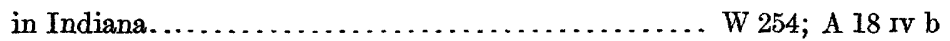
in Kansas....................... W 273; B 338, 530 b; GF 206 in Kentucky............................... B $661 \mathrm{~d}$; GF 184 in Louisiana................................. B 282, $530 \mathrm{~b}$ in Maryland........................................ 258 in Massachusetts................................. W 114 in Míchigan ....................W 30, 31; B 530 b; GF 205 in Minnesota . ...................................... M 25 in Missouri.......................................... 206 in Nebraska . . . . . . . . . . . . . . . . . . . . . . . . . . . . . . 29 in Nevada.......................... W 423; B 530 r, $540 \mathrm{n}$ in New Jersey ........................................ W 258 in New Mexico.................................. W 275, 343 in New York .................................. $530 \mathrm{~b}$ in North Carolina.................................... W 258 in North Dakota................................... M 25 in Ohio.................... W 259; A $18 \mathrm{rv} \mathrm{b;} \mathrm{B} 530$ b; GF 184 in Oklahoma ............................ 148; GF 122 
Quality of ground water regarding salt, in Pennsylvania ......... B 300; GF 144 in Rhode Island ..................................... W 114 in South Carolina........................... $258 ; \mathbf{P} 90 \mathbf{h}$

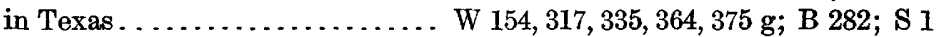
in Virginia...................................... $258 ; \mathrm{S} 8$ in West Virginia . . . . . . . . . . . $530 \ldots \ldots \ldots \ldots \ldots \ldots$ b; GF 184 in Wyoming........................................ 364 silica. W 240, 273, 338; B 330, 491, 616 silver. P 94 sodium ................... . W 254, 259, 273, 338, 341, 398; MR 1911 in in specific gravity . . . . . . . . . . . . . . . . . . . . . . . 47

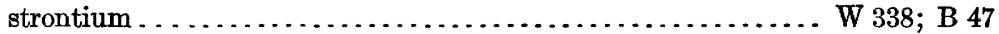
sulphates................ W 254, 259, 273, 275, 338, 341, 343, $345 \mathrm{~g}, 398$; B 330, 491, 616, 653; MR 1911 in n, 1916 sulphides......................... W 338, 345 g; MR 1911 n n, 1916 sulphites............................................ 47 sulphuraria . . . . . . . . . . . . . . . . . . . . . . . . . . . . . 338 suspended matter..................... 254, 258, 259, 341, 398; $\mathbf{\text { S }} 3$ temporary hardness. See Quality of ground water regarding hardness.

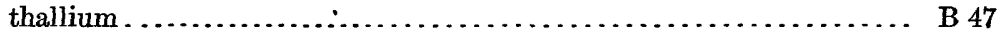
thiosulphites . . . . . . . . . . . . . . . . . . . . . . . . . .

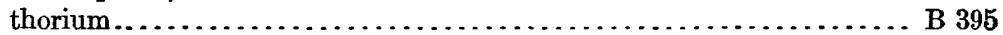

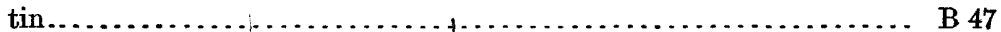

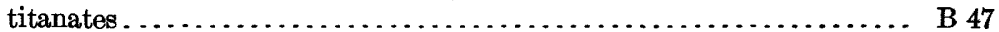
turbidity . . . . . . . . . . . . . . . 3

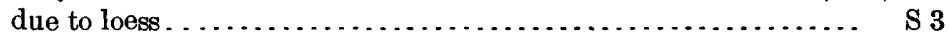
uranium............................................. B 395

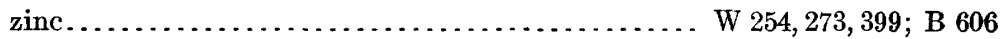
relation of, to carbonate deposits......................... 9 d to concentration............................................. 1911 In $\mathbf{n}$ to deposits of hot springs. .............................. A 9 d

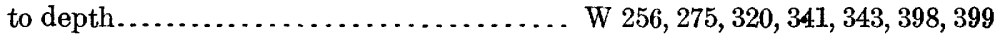
to diffusion . . . . ....................................... 47 to geologic formations........W $254,256,259,273,275,293,320,335,338,341$, 343, $345 \mathrm{~g}, 375 \mathrm{~d}, \mathrm{~g}, 398,399$, 423; A $14 \mathrm{In} \mathrm{b;} \mathrm{P} 90$ h; B 479; 653; S 9 to gypsum beds . . . . . . . . . . . . . . . . . . . . . . . . . . . . 275 to health......... W 31, 164, 195, 233, 254, 259, 293, 335, 338, 341, 343, 398; A 14 II b; P 46; MR 1911 n, 1913 II h to occluded sea water................................ W 398

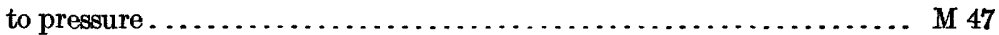
to reactions with adjacent materials............... B 330,491, 616, 653 to silica deposits.................................. 9 d to sodium carbonate from igneous rocks...................W 320 in Cretaceous formations. ....................... W 256,343 to soil alkali.............................. W $320,343,398,423$

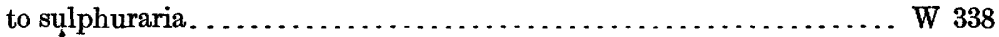
to temperature ..................................... 47 to underground circulation......................... W $320 ; \mathbf{M} 47$ to vegetation. . . . . . . . . . . . . . . . . . . . . . . . . W 343,423 to volcanism . . . . . . . . . . . . . . . . . . . . . . . . B $330,491,616$ to water table.................................. W 320,343 to well casings..................................... W 293 


R.

Racine limestone, water in, in Wisconsin......................... 140

Radioactivity of ground water, bibliography of.................... $1913 \mathrm{~m} \mathrm{~h}$

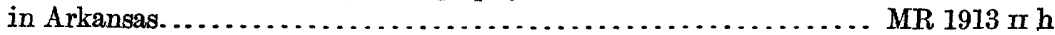

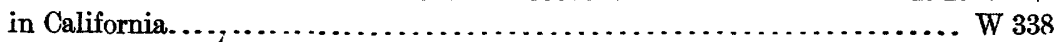

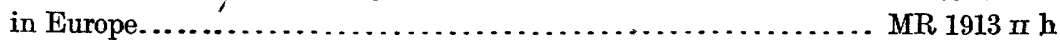

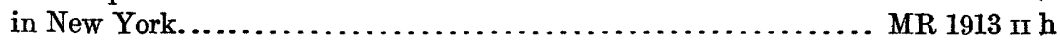

in Yellowstone National Park..................... B 395; MR 1913 in $\mathrm{h}$

measurement of................................ B 395; MR $1913 \mathrm{~m} \mathrm{~h}$

relation of, to temperature............................... 395

thermal, in Europe....................................... B 395

in Yellowstone National Park.................................. 395

Rafter, G. W., Water resources of New York......................... W 25

Rainfall. See Precipitation.

Rams, hydraulic............................... W 1,254,256,258; S 7

Ransome, F. L., Bisbee folio, Ariz............................ GF 112

Globe folio, Ariz........................................ GF 111

Silverton folio, Colo........................................ GF 120

Raritan formation, water in, in Atlantic Coastal Plain................ B 138

water in, in Maryland........................................ 204

in New Jersey .......................... 106; GF 157, 162, 167

in New York............................................ 157

in Pennsylvania.................................. 106; GF 167

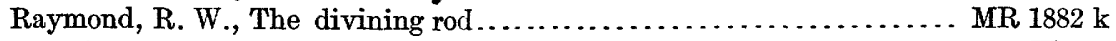

Reaction coefficients in water analyses.......................... W 274

Recharge of ground water. See Absorption, Origin, and Quantity.

Reciprocating pumps............................... 14, 41,398

Records of springs. See Springs.

Records of wells. See Well records.

Recovery of ground water. See Irrigation, Power for pumping, Public water supplies, Pumps, Pumps and other lifting devices, Well construction, and Wells.

Red beds, springs in....................................... 148 water in, in Colorado............................ A 21 rv c; P 52 in Kansas................................ 6 6,$273 ; \mathrm{A} 21 \mathrm{rv}$ c in Nebraska.................................... 21 iv $c$ in New Mexico.............................. $123 ;$ A $21 \mathrm{rv} \mathrm{c}$ in Oklahoma............................... 148; A $21 \mathrm{rv} \mathrm{c}$ in Texas................................. 154, 191; A 21 rv c in Wyoming....................................... 21 c See also specific formations.

Red clastic series, water in, in Minnesota.................. 256; GF 201

Redbank formation, water in, in Atlantic Coastal Plain................. B 138

Reeds, C. A., Water resources of the East St. Louis district, Ill ........... S 3

Renk, on flow of air through soils........................... A $19 \mathrm{Ir} b$

Reservoirs for well water.................................... $1,5,20,343$

Reservoirs, underground, types of ........................... B 319

See also specific formations and structures.

Residual soils, water in.

Return waters. See Discharge of ground water.

Rhabdomancie. See Water witching.

Rhode Island, areas', all of State.

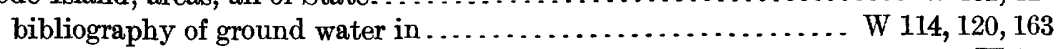

law relating to ground water in ................................. 152 
Rhode Island, mineral waters in . B 32; MR 1885-1916 normal chlorine in W 114, 144 quality of ground water in W 102; B 32 spring records for B 32 well records for. W 61,$149 ; \mathrm{B} 264,298$

Rice, irrigation of, with ground water in Arkansas. . . . . . . . . . . . . . . . . W 399) irrigation of, with ground water in Louisiana.................. W 101

with ground water in Texas. . . . . . . . . . . . . . . . . . . . 71

Richards, R. W., Bull Mountain coal field, Mont. . . . . . . . . . . . . . . . . B 646

Richardson, G. B., Castle Rock folio, Colo.......................... GF 198

El Paso folio, Texas. . . . . . . . . . . . . . . . . . . . . . . . . . . . . GF 166

Indiana folio, Pa......................................... GF 102

Reconnaissance in trans-Pecos Texas.......................... S 1

Underground water in Sanpete and central Sevier valleys, Utah.......W 199 Underground water in the valleys of Utah Lake and Jordan River, Utah. . W 157

Van Horn folio, Texas..................................... GF-194

Richmond formation, water in, in Kentucky...................... W 233

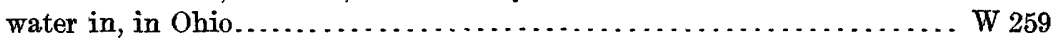

Ripley formation, water in, in Georgia.................... W 341 ; 44

water in, in Illinois. . . . . . . . . . . . . . . . . . 164

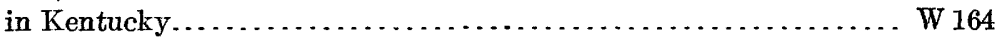

in Mississippi....................................... 159

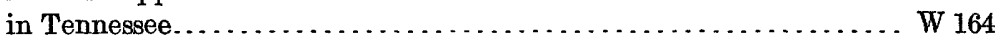

Rivers. See Streams.

Rogers, G. S., Chemical relations of oil-field waters in San \oaquin Valley, Cal.. B 653 Roiliness of well water due to storms............................ W 256

Rome, ancient, mineral water used in ......................... A 14 In b

Roubidoux sandstone, water in, in Missouri. . . . . . . . . . . . . . . . . . W 195

Rubidium in ground water............................... B 47

Russell, I. C., Ann Arbor folio, Mich............................. GF 155

Artesian basins in Idaho and Oregon .......................... 78

Geological reconnaissance in central Washington.................. B 108

Geology and water resources of central Oregon ................... B 252

Geology and water resources of Nez Perce County, Idaho.......... W 53,54

Geology and water resources of the Snake River Plains of Idaho......... B 199

Reconnaissance in southeastern Washington.................... W 4

Russia, bibliography of ground water in...................... 163

S.

Sabine formation, water in, in Arkansas.................... $114 ; \mathbf{P} 46$

water in, in Louisiana. . . . . . . . . . . . . . . . . . . . . $114 ; \ldots \ldots \ldots \ldots$

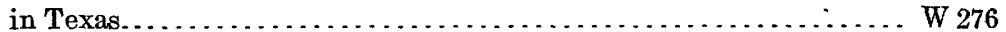

See also Wilcox formation.

Sackett, R. L., Disposal of strawboard and oil-well wastes............... W, 113

Sahara desert, bibliography of ground water in.................... W 163

St. Clair black shale, water in, in Michigan........................ W 30

St. Lawrence formation, water in, in Iowa....................... W 293

water in, in Minnesota.................................... 256

St. Louis limestone, water in, in Illinois......................... W 114

water in, in Indiana. . . . . . . . . . . . . . . . . . . . . . . . . . 114

in Iowa ... . . . . . . . . . . . . . . . . . . . . . . . . . . . .

in Missouri.......................................... W 195 


Schwennesen, A. T., Ground water for irrigation near Enid, Okla. ........ W 345 b

Ground water for irrigation near Oklahoma City, Okla............ W $345 \mathrm{~d}$ Ground water in Animas, Playas, Hachita, and San Luis basins; N. Mex.. W 422 Ground water in Quincy Valley, Wash...................... W 425 e Ground water in San Simon Valley, Ariz.-N. Mex............... W 425 a Pumping tests in Luna County, N. Mex ................. W 345 c; B 618 Screens in wells. See Well screens.

Sea water, confining beds for artesian water produced by effect of, on ground water near coast. incursion of.

Sea water in rocks. See Connate water.

Seaman bucket lift. ........................................ 14

Secondary alkalinity of ground water:.............W 338; P $90 \mathrm{~h} ; \mathrm{B} \mathrm{479,606}$

Secondary salinity of ground water. See under Quality, Salinity, and Salt.

Sections of wells. See Well records.

Sediment in ground water.

Sedgwick, T. F., Report of Water Commission of Hawaii.

Seed-bag packers for wells W 54, 118; A 5 c

Seelheim,- on flow of water through soils.

A $19 \mathrm{n} \mathrm{b}$

Seepage from reservoirs, movements of ground water in relation to in California W 18, 58, 294 in Colorado. W $9,50,358$ in Nebraska. B 140 in Nevada.

See also Discharge and Movements.

Seepage ditches. See Infiltration ditches and tunnels.

Sellersburg limestone, water in, in Indiana........................ W 254

Selma chalk, water in, in Alabama............................... S 2

Serpentine, water in.......................................... 157

Settlement, fluctuation of water table in relation to................... W 155

Seymour formation, water in, in Texas.......................... W 317

Shadoofs for lifting water..................................... W 1

Shakopee dolomite, water in, in Minnesota.................... W 256

Shale, drainage into wells in.................................. W 258 imperviousness of...................................... W 6 water in ......................... W 110, 232, 233, 254, 257, 259, 425 b

Sharon conglomerate, water in, in Ohio....................... $19 \mathrm{rv} \mathrm{b}$

Shattuck, G. B., Patuxent folio, Md.-D. C...................... GF 152

St. Marys folio, Md.-Va.................................. GF 136

Shaw, E. W., Belleville-Breese folio, Ill....................... GF 195

Galena-Elizabeth folio, Ill.-Iowa............................ GF 200

Irvine oil field, Estill County, Ky............................ 661 d

Murphysboro-Herrin folio, Ill.............................. GF 185

Tallula-Springfield folio, Ill................................ GF 188

"Sheet water" .................................... W 29, 154; A 16 I f

Shell marl, water in........................................ W 319

Shenandoah limestone, water in, in Pennsylvania.................. GF 162

Shepard, E. M., Springs of Decaturville dome, Mo.................. W 110

Underground waters of Missouri........................... W 114, 195

Well and spring records for Missouri......................... 102

Sherzer, W. H., Detroit folio, Mich......................... GF 205

Shooting of wells............................... W 223, 255, 257 
Siebenthal, C. E., Geology of the Laramie Basin, Wyo................... B 364 Geology and water resources of the San Luis Valley, Colo................ W 240 Joplin district folio, Mo.-Kans............................ GF 148 Laramie-Sherman folio, Wyo............................ GF 173 Origin of the zinc and lead deposits of the Joplin region .............. B 606 Signs of ground water................................ 224, 423; B 308 Silica in ground water........................ W 240, 273, 338; B 330, 491, 616 Sills, relation of, to springs................................. 400 b Silurian formations, water in, in Alabama.................. W 114; GF 175 water in, in Arkansas.................................... W 114 in Georgia........................................ W $114 ; \mathrm{S} 4$ in Illinois........................ W 114; A $17 \mathrm{~m} \mathrm{~h} ; \mathrm{M} \mathrm{38;} \mathrm{B} 506$ in Indiana............................ W 113, 114, 254; A $18 \mathrm{rv}$ b in Towa............................................ 114, 145, 293

in Kentucky...................................... 114,233

in Maine............................................ 149

in Maryland................................... 110; GF 179

in Michigan ....................... W 30, 31, 114, 182-183; GF 205

in Minnesota....................................... 114, 256

in Missouri....................................... 114, 145, 195

in New York....................................... 114

in Ohio................... W 91, 114, 259; A 18 rv b, 19 rv n; GF 197

in Pennsylvania............................. W 110, 114; GF 179

in Tennessee....................................... W 114

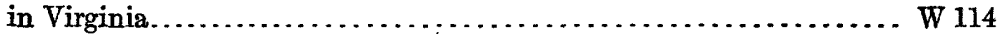

in West Virginia.............................. W 114; GF 179

in Wisconsin............................... W 114; GF 140; 5

See also specific formations.

Silver in ground water.............................. P 94; B 529, 625

Simpson, H. E., Underground water resources of Iowa................... W 293

Singing wells................................................... 29

Sink holes, absorption of water by....................W $319 ;$ A 16 n f; S 4

in gypseous deposits............................. W 343; A 21 rv c

natural wells produced by............................. A 21 rv c

pollution by ................................ W 233, 255, 258; S 3

Sinking of wells. See Well construction.

Sioux quartzite, water in, in Towa............................ W 293

water in, in Minnesota...................................... W 256

Siphon action in drainage wells . ........................... 145, 258

Siphon elevators for lifting water.................................. W 1

Siphons in wells................................ 254, 320, 374, 397 .

Sizing tests for water-bearing materials............................, P 44

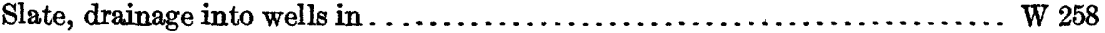

water in............................................. 223, 255, 258

Slichter, C. S., Approximate method of measuring flow of wells .......... W 110

California or "stovepipe" method of well construction .............. W 110

Description of underflow meter............................. W 110

Ground waters of Rio Grande Valley, Tex.-N. Mex ................ W 141

Measurements of underflow .................................. W 140

The motions of underground waters.......................... 67

The underflow in Arkansas Valley in western Kansas................ W 153

The underflow of the South Platte Valley..................... W 184

Theoretical investigation of the motion of ground waters........... A 19 n c

Underground water resources of Long Island, N. Y................ P 44

$56122^{\circ}-18$ - wSP $427-10$ 
Smith, A. G., Report of water commission of Hawaii .............. S 12

Smith, E. A., Underground water resources of Alabama ................ S 2

Underground water in Alabama . . ........................... W 114

Well and spring records for Alabama........................ 102

Smith, G. O., Ellensburg folio, Wash........................... GF 86

Geology and water resources of a portion of Yakima County, Wash...... W 55

Mount Stuart folio, Wash .................................. 106

Penobscot Bay folio, Maine ............................... GF 149

Water from glacial gravels near Augusta, Maine................. W 145

Water resources of the Portsmouth-York region, N. H.-Maine........ W 145

Smith, W. S. T., Edgemont folio, S..Dak.-Nebr.................... GF 108

Joplin district folio, Mo.-Kan........................... GF 148

Water resources of the Joplin district, Mo.-Kan . . . . . . . . . . . . W 145

Well and spring records for Joplin and vicinity, Mo.............. . 102

Soap consumption by water........................... 256,274

methods of calculating...................................... 274

Soap making, quality of water in relation to........................... 233

Sodium in ground water.... W 236, 254, 259, 273, 338, 341, 398; B 47; MR 1911 in n

Softening of water.......................... $254,274,293,315,398$

Softeners required for water, methods of calculating................. W 274

Solids in ground water. See Analyses, Analysis, and Quality.

Solution caverns, water in .............................. W 114; B 319

Solutions comprising ground water, theories and laws relating to..... B 606; M 47

Solutions for water analysis............................... 236

Source of ground water. See Absorption of water and Origin of ground water.

South Australia, bibliography of ground water in ................. 163

South Carolina, areas, Aiken County.......................... B 298

areas, all of State........................................... 114

Barnwell County ..................................... B 298

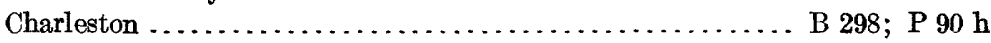

Coastal Plain ....................................... B 138

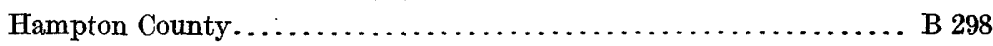

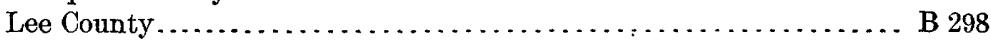

Orangeburg County . . . . . . . . . . . . . . . . . . . . . . 298

Pisgah quadrangle ........................................ GF 147

artesian water in .................................... $138 ;$ P $90 \mathrm{~h}$

bibliography of ground water in................. W 61, 114, 120, 149, 163

blowing wells in........................................... 258

mineral waters in ............................. B $32 ;$ MR 1883-1916

quality of ground water in ..............W 258, 364; B 32, 138; P 90 h

spring records for ........................................... B 32

- well records for.................................. 61,$149 ;$ B 298

South Dakota, areas, Aberdeen quadrangle.................... GF 165

areas, Aladdin quadrangle................................... GF 128

Alexander quadrangle................................. GF 100

all of State.................................... $227 ; \quad$ P 32

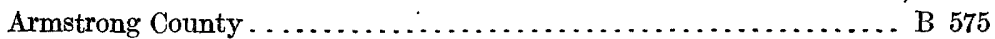

Beadle County ........................................... B 298

Belle Fourche quadrangle............................ GF 164

Black Hills region....................... W 428; A 21 rv b; P 65

Byron quadrangle...................................... GF 165

Cheyenne River Indian Reservation ....................... B 575

Corson County . ..................................... 575

De Smet quadrangle . . . . . . . . . . . . . . . . . . . . . . . . . . . 114 
South Dakota, areas, Dewey County............................ B 575 eastern................................ A $17 \mathrm{~m} \mathrm{g,} 18 \mathrm{rv} \mathrm{c}$ Edgemont quadrangle .............................. GF 108

Flk Point quadrangle.................................. GF 156

Huron quadrangle .................................. GF 113

James River valley . . . . . . . . . . . . . . . . . . . . . . . . 90

Miner County.................................... B 298

Mitchell quadrangle................................. GF 99

Newcastle quadrangle.............................. GF 107

Northville quadrangle................................. GF 165

northwestern.......................................... B 627

Oelrichs quadrangle................................... GF 85

Olivet quadrangle....................................... GF 96

Parker quadrangle................................. GF 97

Redfield quadrangle................................ GF 165

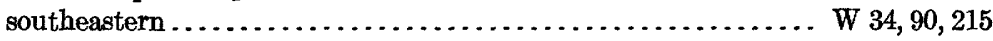

southwestern..................................... $21 \mathrm{rv} \mathrm{b}$

Standing Rock Indian Reservation...................... B 575

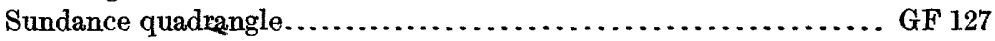

Ziebach County........................................... B 575

artesian springs in................................. 90

artesian water in ................ W 34, 90, 227, 428; B 575; A 21 rv b;

P 32, 65; GF 85, 96, 97, 99, 100, 107, 108, 113, 114, 128, 156, 164, 165

bibliography of ground water in................. W $61,120,149,163$

irrigation with ground water in . ................ A $16 \mathrm{~m} \mathrm{e}, 17 \mathrm{mg}, 18 \mathrm{Iv} \mathrm{c}$

law relating to ground water in........................W 122; S 3

mineral waters in .......................... B 32; MR 1883-1916

quality of ground water in ................................. 32 ;

A $17 \mathrm{~m} \mathrm{~g}, 21 \mathrm{rv} \mathrm{b}$; P 32; GF 97, 99, 100, 107, 108, 113, 164, 165

spring records for.................................... B 32

well construction in .......................... W 34,227 ; GF 96

well records for............................ W 61,$149 ;$ B 264,298

Spain, bibliography of ground water in..................... 163

Spearfish formation, water in, in South Dakota................ GF 107

water in, in Wyoming....................................... 107

Specific capacity of wells....... W 140, 141, 153, 400 b, 425 b; A 19 In b; P 44; S 11 principles of................................ $140 ;$ A $19 \mathrm{n} \mathrm{b}$

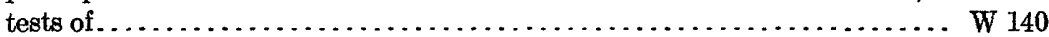

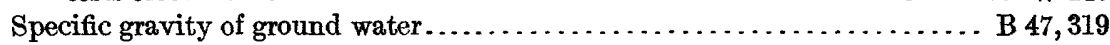

Spencer, A. C., Franklin Furnace folio, N. J.................... GF 161

Spergen limestone, water in, in Missouri......................... 195

Spreading of flood waters in California........................... $\mathrm{S} 6$

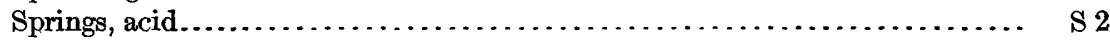

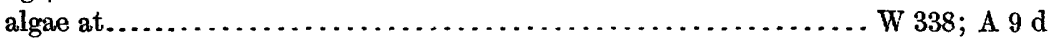

alluvium and bedrock contacts causing ................... W 380

artesian.......................... 255, 278, 338; A 21 Iv c; S 8

barriers causing.............................. $320,343,423$

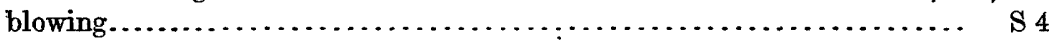

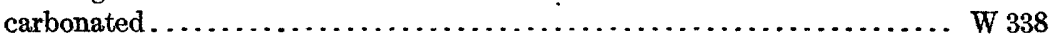

changes in character of water from . . . . . . . . . . . . . . $330,491,616$

classification of..................... W 114, 223, 255, 338, 365; A 14 II b

coal beds causing . . . . . . . . . . . . . . . . . . . 447,627

deposits of $\ldots \ldots \ldots \ldots \ldots \ldots \ldots \ldots \ldots \ldots$. W 338; A 9 d; P 53; B 330, 491, 616 
Springs, depths of pools at

$\mathrm{W} 277,423$

faults in relation to W 142, 199, 232, 277, 338, 365, 380, 423;

A 14 II b, 18 m b; GF 199

fissure. W 114, 255; A 18 m b; B 199; S 9 fluctuations in discharge of. W 277 fountain, artificial...................................... 145 geyser............................................ W 114, 365 artificial. . . . . . . . . . . . . . 145

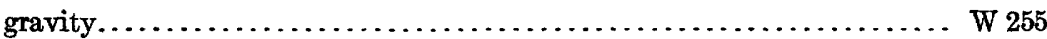
impervious floors causing. . . . . . . . . . . . . . . . . . . 67,380 improvement of . . . . . . . . . . . . . . . . . . . 255,$380 ;$ B 252; S 8 in Alabama............ W 98; A $14 \mathrm{n} \mathrm{b}$; B 32; MR 1883-1916; GF 175; S 2 in Alaska. . . . . . . . . . . . . . . . . . . . W 314, 418; B 32; MR 1883, 1884 in Arizona. .......... W 320, 380; A 14 п b; B 32; MR 1883-1916; GF 111,129 in Arkansas.

W 102, 114, 145, 364;

A 14 II b; P 46; B 32; MR 1883-1916; GF 122, 154 in California.

W $39,142,181,219,224,251,278,300,338,364$; A 14 II b; B 32, 308; MR 1883-1916; GF 39, 66, 101 in Colorado...................................... W 240, 364;

A 14 II b, 16 II f, 17 In f; B 32, 350; MR 1883-1916; GF 135, 153, 203 in Connecticut...... W 102, 114, 232, 374, 397; A 14 II b; B 32; MR 1883-1916 in Delaware................................ B 32; MR 1883-1916 in District of Columbia.................... W 114; B 32; MR 1896-1914 in Florida................. W 102, 114, 319; A 14 Ir b; B 32; MR 1883-1916 in France. A $14 \mathrm{Ir} \mathrm{b}$ in Georgia W 36, 102, 114, 127, 145, 168, 197, 341; A 14 Ir b; B 32; MR 1883-1916; GF 187; S 4 in Hawaii............................ W 77, 318, 336, 373, 430, 445 in High Plains.................................... 21 vr c in Idaho W $54,78,85,135,393$;

A 14 II b; B 32, 199; MR 1883-1916; GF 45, 103, 104 in Illinois. W 114, 364; A 14 II b; B 32, 438; MR 1883-1916; GF 105, 200; S 3 in Indiana........ W 114, 254; A 14 II b, 18 IV b; B 32; MR 1883-1916; GF 105 in Iowa.......... W 145, 293; A 14 II b; B 32; MR 1883-1916; GF 145, 156, 200 in Kansas....................................... 6,145 ; A 14 II b, 16 II f, 21 Iv c; B 32, 238; MR 1883-1916; GF 148, 159, 206 in Kentucky. A. $14 \mathrm{II} \mathrm{b} ; \mathrm{B} \mathrm{32,661 \textrm {d }}$ in Louisiana................... W 114; A $14 \mathrm{~m} \mathrm{b;} \mathrm{P} \mathrm{46;} \mathrm{B} \mathrm{32;} \mathrm{MR} \mathrm{1883-1916}$ in Maine.. W 102, 114, 223, 258, 364; A 14 m b; B 32;MR 1883-1916; GF 149, 158, 192 in Maryland....... W 114; A 14 I b; B 32; MR 1883-1916; GF 152, 160, 179, 204 in Massachusetts............. W 102, 114, 145; A 14 II b; B 32; MR 1883-1916 in Michigan........... W 102, 114, 170, 182, 183; B 32; MR 1883-1916; GF 205 in Minnesota............. W 102, 114, 256; B 32; MR 1883-1916; GF 117, 201 in Mississippi. . . . . . . . . . . . . . . . . . . . . W 114; B 32; MR 1883-1916 in Missouri................ W 99, 102,110, 114, 131, 145, 173, 195, 209, 364; B 32, 438; MR 1883-1916; GF 148, 206 in Montana . . . ..... W 130, 221, 326, 400 b; B 32, 646; MR 1883-1916; GF 24, 56 in Nebraska............................... W 12, 39, 70, 184, 230;

A 16 II f, 19 Iv c; P 17; B 32; MR 1905-1916; GF 108, 156

in Nevada W $85,133,224,360,364,365,375$ d, 389, 390, 423;

B 32, 308; MR 1883-1916

in New Hampshire. W 102, 114; B 32; MR 1883-1916 
Springs in New Jersey ............ W 110, 114; B 32; MR 1883-1916; GF 161, 167, 191 in New Mexico........ W 158, 188, 343, 364, 380; B 32, 618; MR 1883-1916; GF 199 in New York..........W 102, 114, 145, 364; P 44; B 32; MR 1883-1916; GF 169 in New Zealand.

A $9 \mathrm{~d}$ in North Carolina........... W 114, 364; B 32; MR 1883-1916; GF 124, 147, 151 in North Dakota...................... B 32, 575; MR 1883-1916; GF 117 in Ohio........... W 91, 114; A $14 \mathrm{~m} \mathrm{~b}, 18 \mathrm{rv} \mathrm{b;} \mathrm{B} \mathrm{32;} \mathrm{MR} \mathrm{1883-1916;} \mathrm{GF} 197$ in Oklahoma......... W 99, 148, 209, 247, 364; B 32; MR 1883-1916; GF 122, 132 in Oregon........ W 78, 214, 220, 231, 250, 251, 252, 271, 310, 360, 370, 390, 391, 394;

A 14 II b; B 32, 252; MR 1883-1916; GF 103 in Pennsylvania .......... W 106, 114; A 14 II b; B 32, 300, 447; MR 1883-1916; GF 121, 123, 133, 160, 167, 170, 172, 174, 179, 189

in Rhode Island W 102; A 14 II b; B 32 in South Carolina. in South Dakota W 114; A 14 II b; B 32; MR 1883-1916; GF 147 W 34, 90; A 14 II b, 21 rv b; B 32, 575, 527; MR 1883-1916; GF 99, 100, 108, 113, 114, 156 in Tennessee....... W 114, 164, 364; A 14 II b; B 32; MR 1883-1916; GF 124, 151 in Texas.............. W 28, 37, 66, 71, 84, 105, 132, 154, 174, 191, 288, 317, 335; A 14 II b, 18 II b; B 32, 131, 140; MR 1883-1916; GF 42, 64, 183; S 1 in Utah.. W 133, 157, 199, 212, 217, 277, 359, 360, 364, 389, 390; B 32; MR 1883-1916 in Vermont.................... W 102, 114; A 14 II b; B 32; MR 1883-1916 in Virginia................... W 114, 364; A 14 II b; B 32; MR 1883-1916; S 8 in Washington ................ W 111, 118, 294, 316, 332, 362, 425 e; A 14 n b;

B 32; M R 1883-1916; GF 86, 106 in West Virginia......... W 114, 145; B 32; A 14 II b; MR 1883-1916; GF 160, 179 in Wisconsin....... W 114, 145; A 14 II b; B 32; MR 1883-1916; GF 140, 145; S 9

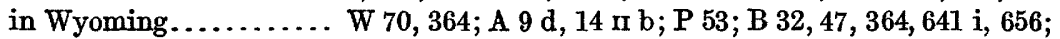

MR 1883-1916; GF 173 in Yellowstone National Park............. W 364; A 9 d; 14 II b; B 47, 395

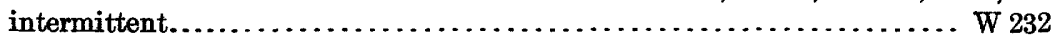

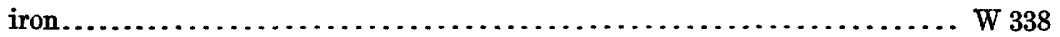
irrigation water from ......................... W 277, 365; GF 104 joints in relation to.................................... 221,232 joints in sandstone causing............................. W 221 knolls produced by.......................... W 277, 320, 343, 365, 423 large.. W 27, 28, 36, 37, 85, 98, 99, 127, 130, 131, 145, 158, 168, 173, 177, 209, $214,221,251,271,277,288,298,300,319,326,332,338,360$, $362,365,370,391,394 ;$ A 14 II b, 18 II b; B 131, 140, 641 i

magnesic.................................................... 338

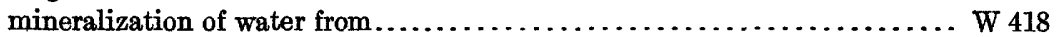
mounds produced by $\ldots \ldots \ldots \ldots \ldots \ldots \ldots \ldots \ldots \ldots \ldots$ W $277,320,343,365,423$ ocherous deposits of $\ldots \ldots \ldots \ldots \ldots \ldots \ldots \ldots \ldots \ldots \ldots \ldots \ldots$ B $330,491,6 \ldots \ldots$

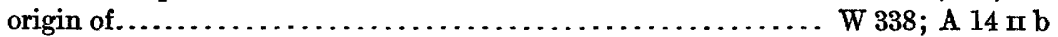

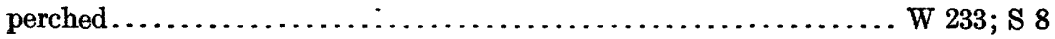

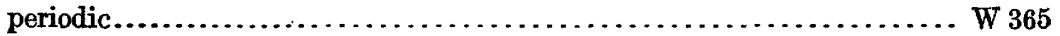

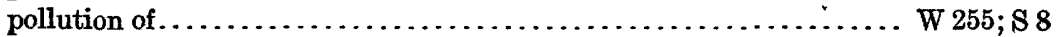

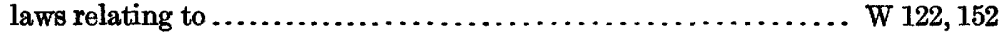
pool......................................... $221,277,365,423$ radioactivity of ............................ B 395; MR 1913.II h salt......................... W 148, 154, 338, 343; M 25; GF 122, 129 seepage............................................ 255 siliceous deposits of $\ldots \ldots \ldots \ldots \ldots \ldots \ldots \ldots \ldots \ldots$ W 338; A $9 \mathrm{~d} ; \mathrm{B} 330,491,616$ sills in relation to....................................... $400 \mathrm{~b}$ 
Springs, streams in relation to

A 14 II a

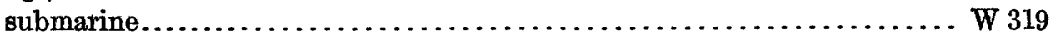
sulphur........................ W 148, 217, 338; B 641 i; GF 101, 154 symbols for, on maps.......................................... 160

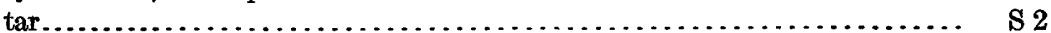
therapeutic value of waters from.............. A $14 \mathrm{~m} \mathrm{~b}$; MR $1911 \mathrm{II} \mathrm{n}, 1916$ thermal, artesian wells in relation to........................ W $400 \mathrm{~b}$ boiling points at various elevations in relation to...............W 338 definition of .................................. 14 Ir b

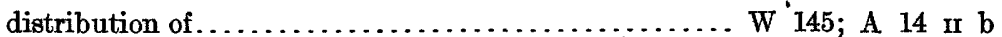

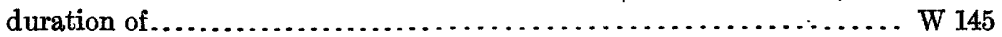
faults in relation to............... W $277,338,365,380 ;$ A 14 II b in Alaska............................................ W 418 in Arizona ................................... $582 ;$ GF 129 in California................... W 142, 181, 338, 364; B 308; GF 39, 101 in Colorado................................. W 364; GF 153 in Idaho................................. $78 ;$ B 199; GF 45, 104 in Montana............................. W 364, $400 \mathrm{~b}$; GF 24, 56 in Nevada............................ W 364, 365, 423; B 308 in New Mexico............................. W 364; GF 199 in New Zealand...................................... $9 \mathrm{~d}$ in Oregon ................................... W 220; B 252 in South Dakota........................ W 145; A 21 rv b; P 32 in southern United States.............................. W 145 in Utah.............................. $217,277,333,364$ in Virginia.............................................. W 364 in West Virginia........................................ GF 179 in Wyoming.............W 364; A $9 \mathrm{~d} ; \mathrm{P} 53 ; \mathrm{B} 32,47,395,641$ i in Yellowstone National Park............. W 364; A 9 d; B 32,47,395 origin of heat in............................... W 338,418; A 14 II b radioactivity of ............................. B 395; MR 1913 ir $h$ topography in relation to ............................... $14 \mathrm{n} \mathrm{b}$ variations in temperature of.......................... A $14 \mathrm{~m} \mathrm{~b}$ volcanism in relation to ...................... W 181; B $330,491,616$ travertine deposited by.................. W 338, 423; B 330, 491, 616; A 9 b tubular................................................. 255

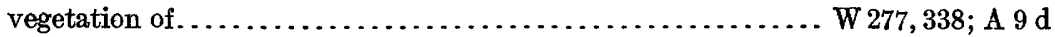
volcanoes in relation to ..................................... 181 yield of, in United States............................ A 14 II b; B 32 See also Discharge of ground water and Mineral waters.

"Spring-stream valleys" in Great Plains......................... A $22 \mathrm{rV}$ c Stabler, 'Herman, Ground water in San Joaquin Valley, Cal............... W 398

Stream waters of western United States........................... W 274 Standards for water analysis. See Analysis.

Starch making, quality of water for........................... 341,398

State wells............................................... 122

Stauffer, C. R., Columbus folio, Ohio............................ GF 197

Steam making. See under Quality of water.

Stephenson, L. W., Coastal Plain of North Carolina..................... S 5

Deep well at Charleston, S. C............................ P $90 \mathrm{~h}$

Geology and ground water of northeastern Arkansas................. W 399

Underground waters of Coastal Plain of Georgia...................... W 341

Water supply of Savannah, Ga.............................. S 11 
Sterilization of water. W 254,315

See also Purification.

Stevens, G. C., Surface water in California, $1912 \ldots \ldots \ldots \ldots \ldots \ldots \ldots \ldots \ldots$. . . . . . . 331

Stockton formation, water in, in New Jersey...................... GF 167

water in, in Pennsylvania.................................. GF 167

Stock, quality of water for.............................. 335,343

Stone, R. W., Elders Ridge folio, Pennsylvania...................... GF 123

Water resources of the Elders Ridge quadrangle, $\mathrm{Pa} . \ldots \ldots \ldots \ldots \ldots \ldots$

Waynesburg folio, Pa....................................... 121

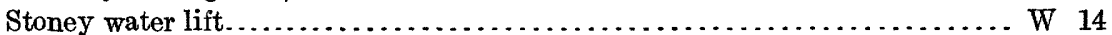

Storage capacity of rocks for water. See Quantity.

Storage of well water............................... W 1,5, 20, 184, 343

Storrs, H. A., Cost of power for pumping irrigation water ................. W 146

Stose, G. W., Apishapa folio, Colo.............................. GF 186

Mercersburg-Chambersburg folio, Pa........................ GF 170

Pawpaw-Hancock folio, Md.- Pa.- W. Va ....................... GF 179

Water resources of the Chambersburg and Mercersburg quadrangles, Pa... W 110

Water resources of the Pawpaw and Hancock quadrangles, W. Va.-Md.-Pa.. W 145

Stovepipe well casings............................... W, 110, 140, 255, 257

Strainers in wells. See Well screens.

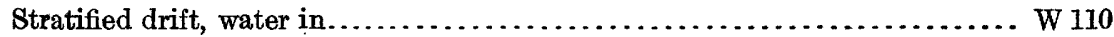

water in, in Connecticut............................. W 232,374, 397

in Texas................................................. 317

See also Glacial drift and Glacial outwash.

Stream deposits. See Alluvium, Valley fill, and Glacial outwash.

Streams, artesian head in relation to.

GF 156

diversion of, by underground channels..................... A 21 IV c

ground water in relation to .......... W 9, 10, 18, 58, 67, 199, 234, 294, $375 \mathrm{~d}, 423$;

A 14 II a, 19 II b

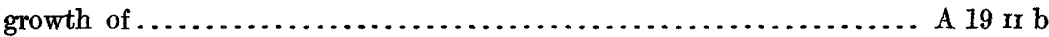

underground....................... W 233,258; A 18 Iv b, 21 Iv c; S 4,8

water table in relation to..................................... 155

Strontium in ground water................................ $338 ;$ B 47

Structure of rocks, thermal springs in relation to.................... A 14 in b

Structural valleys. See Débris-filled basins.

Subalkalinity of ground water.................................... 338

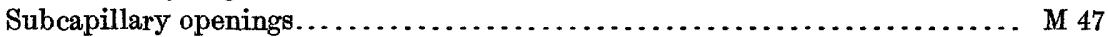

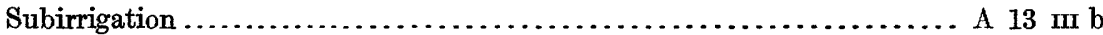

Subsurface dams. See Dams.

Sucking wells............................................... W 258

Suction of pumps................................................. 14

Sugar making, quality of water for ........................ W 30, 341, 398

Sulphates, field tests for...................................... W 151

Sulphates in ground water ........... W 254, 259, 273, 275, 338, 341, 343, $345 \mathrm{~g}, 398$;

B 330, 491, 616; MR 1911 I n, 1916

Sulphide enrichment, ground water in relation to................. B 529,625

Sulphides in rocke, ground-water levels in relation to................ B 529,625

Sulphides in ground water............. W 338, 345 g; MR 1911 In n, 1916; GF 206

Sulphites in ground water................................... B 47

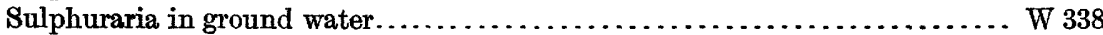

Sundance formation, water in, in Seuth Dakota...................... GF 107

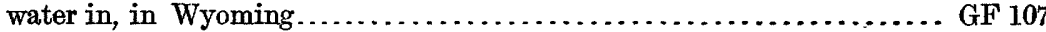

Sunderland formation, water in, in Maryland.................... GF 152

Supercapillary openings................................. M47 
Suspended matter in ground water. W 254, 258, 259, 341, 398; S 3

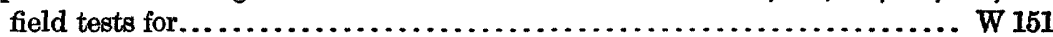

Swamps, water table in relation to.................................... 258

Swartz, C. K., Pawpaw-Hancock folio, Md.- W. Va.-Pa.................... GF 179

Switzerland, bibliography of ground water in.......................W 16 \$ quality of ground water in............................... B 330, 491, 616 radioactive waters in ....................................... 1913 in $h$

Sylvania sandstone, water in, in Michigan................... W 30; GF 205 Symbols for springs on maps...................................... W 160 Symbols for well data on maps..................................... W 160

T.

Taff, J. A., Muscogee folio, Okla.............................. GF 132 Tahlequah folio, Okla.-Ark............................ GF 122

Talbot, A. N., Corrections in determinations of flow from vertical well casings. W 110

Talbot formation, water in, in Delaware........................ GF 137

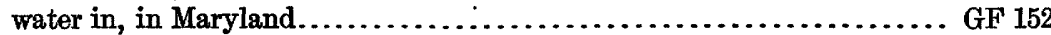

Tallahatta buhrstone, water in, in Mississippi. . ................... W 159

Tampa formation, water in, in Florida.............................. 319

Tanning, quality of water for......................... W 254, 341, 398

Tar springs in Alabama. ...................................... $\mathbf{S} 2$

Tarr, R. S., Water resources of the Watkins Glen quadrangle, N. Y......... W 110 Watkins Glen-Catatonk folio, N. Y............................ 169

Tasmania, bibliography of ground water in . . . . . . . . . . . . . . . W 163

Taylor, F. B., Niagara folio, N. Y............................ GF 190

Water resources of the Taconic quadrangle, N. Y.-Mass.-Vt............ W 110

Taylor marl, water in, in Texas............................... W 276

Taylor, T. U., Irrigation systems of Texas....................... W 71

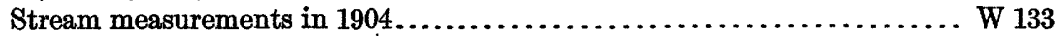

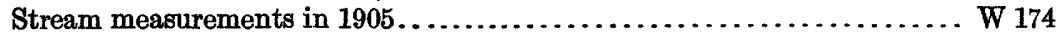

Underground waters of the Coastal Plain of Texas.................. W 190

Water powers of Texas..................................... W 105

Tempany, H. A., on ground water in Antigua..................... $\mathbf{S} 10$

Temperature, artesian flows influenced by.............. W $400 \mathrm{~b} ; \mathrm{A} 5 \mathrm{c}$; B 319 changes of, fluctuations of water table in relation to............. W 155; P 44 movements of ground water in relation to................ A $19 \mathrm{Ir} \mathrm{b;} \mathrm{B} 319$ of artesian water........ W 55, 78, 240, $400 \mathrm{~b} ; \mathrm{A} 18 \mathrm{rv}$ c; B 199, 319; GF 45; S 2 changes in ............................................. 240

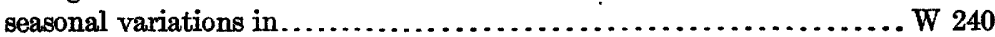
yield of wells in relation to......................W $400 \mathrm{~b} ; \mathrm{A} 5 \mathrm{c}$

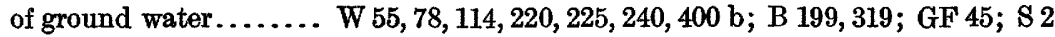
causes that raise............................... W 338; A 14 Ir b density in relation to.................................... B 319 dissolved gases in relation to................................ M 47 dissolved solids in relation to................................ M 47 fluctuations of water table in relation to........................ M 47 irrigation in relation to.................................. W 398

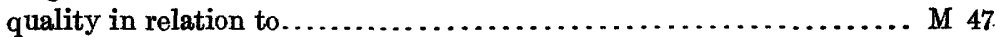
radioactivity in relation to............................. B 395 variations in ..................................... 14 í b volcanism in relation to........................... B $330,491,616$ yield of wells in relation to........................ W $400 \mathrm{~b} ; \mathrm{A} 5 \mathrm{c}$ See also under Springs. 
Temporary hardness of ground water. See under Quality of ground water.

Tennessee, areas, all of State.............................. W 102, 114

areas, Mount Mitchell quadrangle........................... GF 124

Roan Mountain quadrangle........................... GF 151

west of Tennessee River.................................... W 164

artesian water in .......................................... W 164

bibliography of ground water in................. W 114, 120, 149, 163, 164

law relating to ground water in.............................. 152

mine water in .................................... W $364 ;$ B 529

mineral waters in ........................ W 164; B 32; MR 1883-1916 quality of ground water in........W 164, 364; B 32, 330,491, 529,616; GF 124 spring records for......................................... 32 well records for............................... W 61, 149; B 264, 298

wells in, construction and cost of............................ W 164

Tensleep sandstone, water in, in Wyoming......... P 51, 53; B 656; GF 141, 142

Terrell reservoir, water in, in Texas.......................... A 21 vII

Tertiary alkalinity................................. $338 ;$ B 479

Tertiary formations, water in, in Alabama.................... 114 ; S 2

water in, in Antigua.................................... S 10

in Arizona................................................... 380

in Arkansas..................................... 114, 145, 399; P 46

in California......................... 225, 375 a; GF 66, 138

in Colorado........................ A 16 II f, 17 II f, 21 Iv c, 22 Iv c;

M $27 ;$ P 32, 52; B 531 c; GF 71, 135, 198

in Delaware........................ W 114; B 138; GF 137, 162

in District of Columbia............................... GF 152

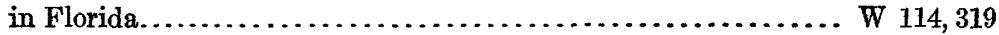

in Georgia............................. W 114, 341; B 138; S 4

in Idaho.................... W 53-54,78; B 199; GF 45, 103, 104

in Illinois........................... W 114, 164; A $17 \mathrm{n} \mathrm{h}$; M 38

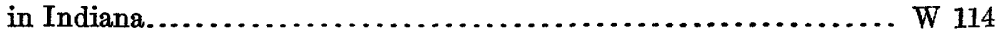

in Kansas.............. W 6, 273, 345 a; A 16 II f, 21 Iv c, 22 Iv c; P 32

in Kentucky........................................ 114, 164

in Louisiana................................ 101,$114 ; \mathrm{P} 46$

in Maryland............. W 114; B 138; GF 13, 23, 136, 137, 152, 182, 204

in Mississippi...................................... 114, 159

in Missouri...................................... 114,195

in Nebraska........................... W 12, 70, 215, 216, $425 \mathrm{~b}$;

A 16 I f, 19 IV c, 21 IV c, 22 IV c; P 17, 32; GF 87,88

in Nevada......................................... W 423

in New Jersey....................... W 114; B 138; GF 137, 162

in New Mexico......................... W 380; A 21 Iv c, 22 Iv c

in North Carolina....................... W 114; B 138;GF 80; S 5

in North Dakota...................................... 181

in Oklahoma....................... W 148, $345 \mathrm{~b} ; \mathrm{A} 21 \mathrm{rv} \mathrm{c}, 22 \mathrm{rv} \mathrm{c}$

in Oregon.....................W 53-54, 78, 220, 231; B 252; GF 103

in Pennsylvania................................ W 144; GF 162

in South Carolina............................... W 114; B 138

in South Dakota.......................... W 227; P 32; B 627

in Tennessee. ...................................... W 114, 164

in Texas.... W 154, 190, 191, 276, 335, $375 \mathrm{~g} ; \mathrm{A} 21 \mathrm{rv}$ c, $21 \mathrm{vIr}, 22 \mathrm{rv}$ c; GF 64

in Utah.......................................... 285 f; P 56

in Virginia..................... W 114; B 138; GF 13, 23, 80, 136; S 8 
Tertiary formations, water in, in Washington

W 53-54, 55, 111, 118, 316, 425 e; B 108; GF 86, 106

in Wyoming $\mathrm{W} 70$;

A 21 IV c, 22 IV c; P 32, 51, 56

See also specific formations.

B 285 f, 364, 425 b, 543; GF 173

Tertiary salinity.

W $338 ;$ B 479

Texas, areas, all of State.

A $11 \pi \mathrm{C}$

areas, Austin and vicinity

W $84,132,174 ;$ B 131

Austin quadrangle................................... GF 76

Bexar County ....................................... B 298

Black Prairie...................................... A 21 vI

Burnet quadrangle. ...................................... GF 183

Cameron County...................................... B 298

Carrizo Springs and vicinity......................... W 66; B 164

Coastal Plain ............................... 190, 335; B 282

Dallas County..................................... B 298

Dimmit County....................................... B 298

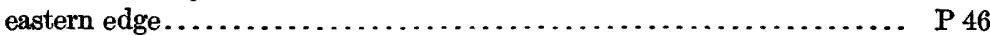

Edwards Plateau.................................... A 18 m b

E1 Paso and vicinity........................ W 140, 141, 343; GF 166

El Paso quadrangle..................................... GF 166

Fort Bend County.................................. B 298

Fort Stockton and vicinity.............................. W 132

Grand Prairie ...................................... A 21 vI

Guadalupe County..................................... B 298

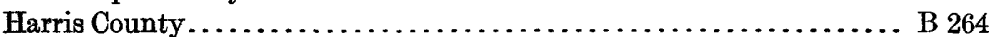

Hays Gounty . . . . . . . . . . . . . . . . . . . . . . . . . . . . . . . B 298

High Plsins................................. A 21 Iv c, 22 IV c

Johnson Gounty..................................... B 298

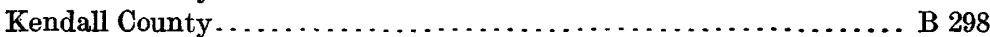

La Salle County ................................... W 375 g

Lampasas County ..................................... B 298

Llano quadrangle ..................................... GF 183

McMullen County ................................... W $375 \mathrm{~g}$

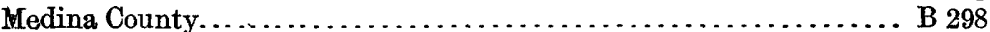

north-central. ...................................... W 317

northeastern ........................................... W 276

Nueces County........................................ B 298

Nueces quadrangle.................................... GF 42

Palo Pinto County .................................. B 621 e

Panhandle, eastern part of ................................ 154

western part of...................................... W 191

Parker County......................................... B 298

Rio Grande Plain. ................................ A 18 In b

Roberts County ..................................... B 298

San Angelo and vicinity ............................ 132

southern. ...................................... 18 II b

trans-Pecos region.....................W $140,141,343 ;$ GF 166; S 1

Toyahville and vicinity ................................ 132

Uvalde quadrangle.................................... GF 64

Van Horn quadrangle................................... GF 194

western.................................... A 21 rv c, $22 \mathrm{rv}$ c 


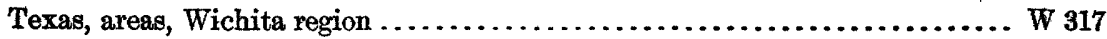

Williamson County................................... B 298

Yellow House basin................................. A 21 Iv c

Zavalla County....................................... B 298

artesian water in....................W 13, 66, 71, 191, 276, 335, 375 g;

A 11 II c, 18 II b, 21 vII; GF 42, 64, 76, 194; S 1

bibliography of ground water in...................W $61,120,149,163$

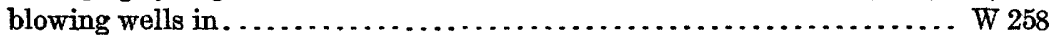

gypsum springs in........................................ 154

irrigation with ground water in......... W $5,13,66,71,154,190,191$, 375 G;

A 11 II c, 16 II e, 21 IV c, 22 IV c

law relating to ground water in.............................. 152 mineral waters in . ........................... B 32; MR 1883-1916

pumping in, cost of..................................... 13,141

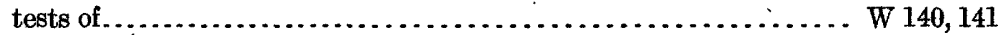

public water supplies in.............. W 13, 190, 335, 343; A 18 II b; GF 166

quality of ground water in ............... $66,141,190,276,317,348,364$;

A 18 Ir b, 21 vII; P 46; B 32; GF 166, 194; S 1

rice irrigation with ground water in............................. W 71

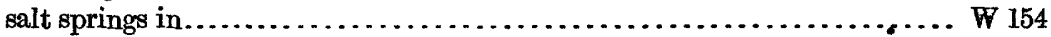

salt water in oil fields of . . . . . . . . . . . . . . . . . . $28 \ldots \ldots \ldots \ldots \ldots \ldots$

"sheet water" in ......................................... W154

spring records for. ............W 28, 37, 84, 105, 132, 174, 288; B 32, 131, 140

underflow measurements in.................................. W 141

well records for............................. W 61, 149; B 264, 298

wells in, cost of. ................................. A 11 n c; B 282

Thallium in ground water........................................ B 47

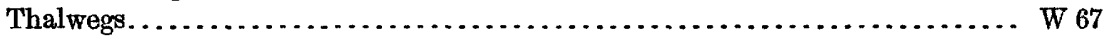

Therapeutic properties of water.. W 31, 164, 195, 233, 254, 259, 293, 335, 338, 341, 398; A 14 Ir b; P 46; MR 1911 m n, 1913 II h

Thermal movements of ground water. ........................... A $19 \mathrm{~m} \mathrm{~b}$.

Thermal springs. See under Springs.

Thomas, A. O., Underground water resources of Iowa................. W 293

Thorium in ground water....................................... B 395

Tides, fluctuations of water table caused by........... W 155, 232; P 44; GF 193 See also Water table.

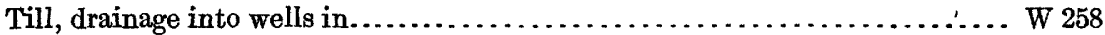

water in .......................................... $114,255,257$

in Connecticut............................... $232,374,397$

in Illinois............................................. 188

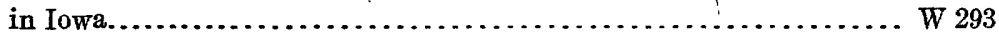

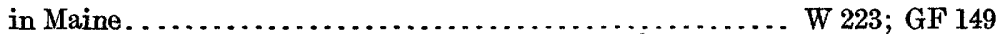

in Minnesota ......................................... 256

in Montana......................................... $400 \mathrm{~b}$

in Nebraska . . . . . . . . . . . . . . . . 156

in South Dakota......................... GF 96, 97, 100, 113, 114, 156

See also Glacial drift.

Tilting troughs for lifting water................................. W 1

Tilton, J. L., Underground water resources of Iowa..................... W 293

Tin in ground water.......................................... B 47

Titanates in ground water....................................... B 47 
Todd, J. E., Aberdeen-Redfield folio, S. Dak........................ GF 165 Alexandria folio, S. Dak..................................... 100

De Smet folio, S. Dak.................................... GF 114

Elk Point folio, S. Dak.-Nebr.-Iowa............................. 156

Geology and water resources of the lower James River valley, S. Dak.... W W 90

Geology and water resources of southeastern South Dakota............. W 34

Huron folio, S. Dak........................................ 113

Mitchell folio, S. Dak..................................... GF 99

Olivet folio, S. Dak.......................................... GF 96

Parker folio, S. Dak.......................................... 97

"Top water" in relation to oil.................................... 658

Topography, thermal springs in relation to................... A $14 \mathrm{~m} \mathrm{~b}$

water table in relation to.................. W 67, 159, 254, 374; $A 19$ п b

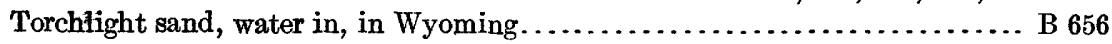

Torpedoing of wells.................................... 233, 255, 257

Transmission constant. ..................................... 67,140

Transpiration......................... W 7, 153, 294, 320, 375 d, 423; S 7

measurements of............................................ 294

Trap rock, water in....................... W 110, 160, 223, 232, 374; B 319

Trautwine, on flow of water through soils....................... A 19 II b

Traverse formation, water in, in Michigan ................. W 30, 114; GF 205

Travertine deposited by ground water....................... 338, 343, 423;

A $9 \mathrm{~d}, 18 \mathrm{II} \mathrm{b}$; B 330, 491, 616; S 3

Travis Peak sand, water in, in Texas........................... GF 42

Trent formation, water in, in North Carolina ...................... S 5

Trenton limestone, water in, in Illinois.................... 506 ; GF 81

water in, in Indiana....................................... 114, 254

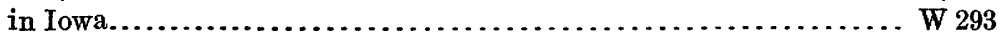

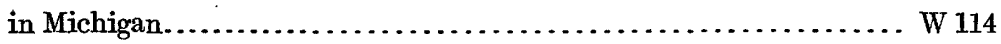

in Ohio................................ W 114; A 19 rv b; GF 197

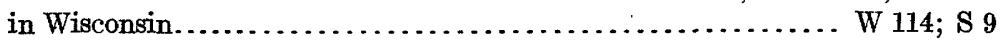

See also Platteville limestone.

Triassic formations, water in, in Arizona..................... W 380; B 435 water in, in Colorado........................... A 16 In f, 17 пा f; P 52

in Connecticut. ............................. W 110, 114, 232, 374

in Kansas......................................... A 16 п $\mathrm{f}$

in Massachusetts .............................................. 110

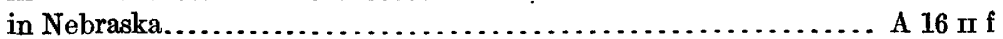

in North Carolina.......................................... 114

in New Jersey ............................. 114; GF 157, 167, 191

in New Mexico.................................. 3 380; B 435

in New York...................................... 114; GF 157

in Pennsylvania.......................... W 106, 114; GF 162, 167

in South Dakota....................................... GF 107, 164

in Texas.......................................... 154, 191

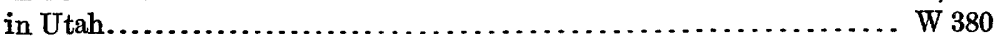

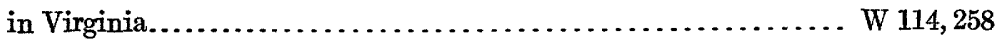

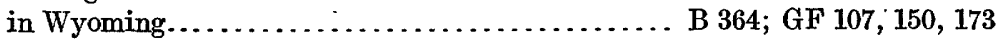

See also specific formations.

Trinity formation, water in, in Arkansas........................ P 46

water in, in Colorado................................ A 16 Ir f

in Kansas........................................... A 16 II

in Nebraska. ...................................... 16 In $\mathrm{f}$

in Texas.......................... W 276; A 21 vII; GF 42, 76, 183 
Triplex pumps.................................................. 14

Troughs, tilting, for lifting water................................. W 1

Trowbridge, A. C., Galena-Elizabeth folio, Ml.-Iowa................... GF 200

Tufa deposited by ground water.. W 338, 343, 423; A 9 d, 18 II b; B 330, 491, 616; S 3

Tuff, water in .................................... W 375 a; GF 138

Tunnels for recovering ground water. See Infiltration ditches and tunnels.

Turbidimeters for testing water................................... 151

Turbidity of ground water............................ W 341, 398; S 3

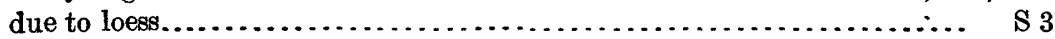

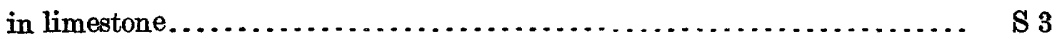

Turkestan, bibliography of ground water in ........................ W 163

Turner, H. W., Marysville folio, Cal............................. GF 17

Tuscahoma formation, water in, in Alabama......................... S 2

Tuscaloosa formation, water in, in Alabama......................... S 2

water in, in Georgia........................................ S 4

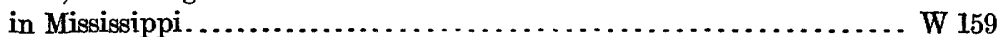

Tuscan tuff, water in, in California.................... W 375 a; GF 138

Tympana for lifting water........................................ 1,29

$\mathrm{U}$.

Udden, J. A., Belleville-Breese folio, Ill......................... GF 195

Geology and mineral resources of the Peoria quadrangle, Ill............ B 506

Underflow, recovery of ..................................... W 380

Underflow dams. See Dams.

Underflow ditches. See Infiltration ditches and tunnels.

Underflow measurements............ W 112, 140, 141, 153, 184, 258; A 19 II b; P 44 cost of ................................................ 112 methods for making................................. 112,$140 ;$ P 44

Underflow meters................................W 112, 140, 141; P 44

See also Movements of ground water.

Underground dams. See Dams.

Underground streams....................W 233, 258; A 18 Iv b, 21 Iv c; S 4,8

Uniformity coefficient of water-bearing materials................ W 67; P 44

United States Geological Survey, ground-water work of, prior to $1904 . \ldots \ldots \ldots$. B 227

United States history, ground water in relation to.................. A 14 II a

Unkpapa sandstone, water in, in South Dakota..................... P 65

water in, in Wyoming.................................. P 65

Upham, Warren, Glacial Lake Agassiz........................ M 25

Uranium in ground water...................................... B 395

Utah, areas, Bear River valley................................. W 333

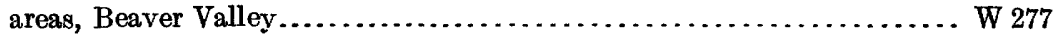

Blue Spring Valley................................. 333

Boxelder County...................................... W 333

Central Sevier Valley.................................... W 199

Curlew Valley..................................... W 333

east-central...................................... B 628

Escalante Desert..................................... 277

Fish Springs Valley................................ 277

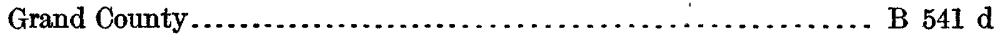

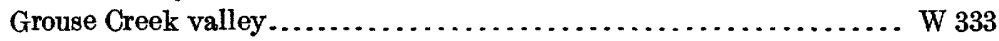

Hansel Valley .......................................... W 333

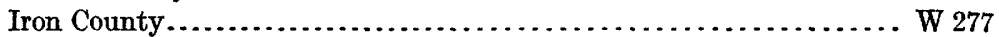

Jordan River valley.............................. W 157 
Utah, areas, Juab County ..................................... W 277

Juab Valley ........................................ W 277

Millard County...................................... W 27t

northern..................................... W 7,333

old River Bed region............................... W 277

Park Valley.............................................. W 333

Parowan Valley........................................ W 277

Pavant Valley....................................... W 277

Round Valley........................................ 277

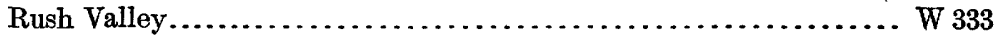

Rush Lake valley ..................................... W 277

Salt Lake County ....................................... B 298

Sanpete Valley...................................... W 199

Sevier Desert.......................................... W 277

Skull Valley........................................ W 333

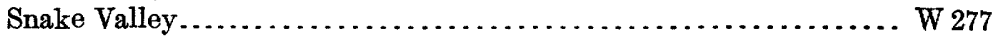

southeastern ............................................. 380

Tintic Valley.......................................... 277

Tooele County........................................ W 333

Tooele Valley ........................................ W 333

Utah Lake Valley .................................... W 157

White Valley...................................... W 277

artesian water in ........................... W 157, 199, 217, 277, 333

bibliography of ground water in .......................... 120,163

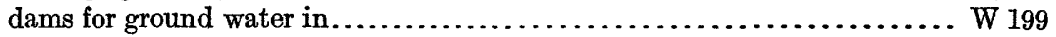

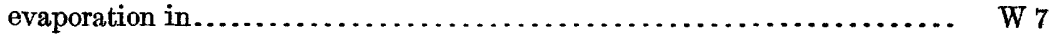

guides to watering places in ............................ W 277, 333

infiltration tunnels in.................................... B 199

irrigation with ground water in................. W 217,277, 333; A 16 и e

law relating to ground water in ............................ 122, 152

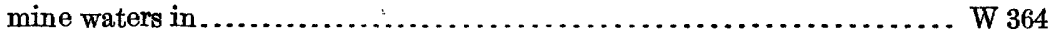

mineral waters in .......................... B 32; M R 1883-1916

quality of ground water in............... W 157, 199, 217, 277, 333, 364; B 32

seepage water in ........................................ W 7

spring records for..................... W 133, 212, 359, 360, 389, 390; B 32

well records for............................... W 61, 149; B 264, 298

Utica shale, water in, in Michigan ............................... W 114

water in, in Ohio........................................... 259

V.

Vacuum gages................................................... 141

Vacuum in pumps, methods of determining....................... W 141

Vadose circulation of ground water................................ B 529

Vadose waters................................... B 330, 491, 529, 616

Valley fill, interbedded with lava, water in.................... W 54; B 199

water-bearing capacity of....................................... 423

water in, diagram showing............................... W 277, 333

early statement in regard to......................... A 13 mi a

in Arizona........... W 104, 136, 320, 375 b, 380, 425 a; B 352; GF 111, 112

in California..................W 89, 137, 138, 139, 142, 219, 222, 225, 278, 294,375 a, h, 398, 400 e; GF 163, 193; S 6, 7

in Colorado .......................................... W 240

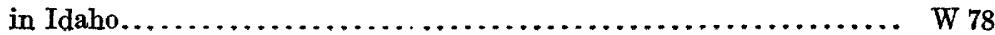


Valley fill, water in, in Montana

$\mathrm{W} 345 \mathrm{~g}, 400 \mathrm{~b}$

in Nevada................................ W 365, $375 \mathrm{~d}, \mathbf{4 2 3}, \mathrm{B} 530 \mathrm{r}$

in New Mexico...................................... W 123,

$158,188,260,275,343,345$ c, 425 a; B 618; GF 199, 207

in Oregon ..............................W 78, 220, 231; B 252

in Texas ................................. 343 ; GF 166, 194; 81

in Utah.............................. $157,199,217,277,333$

See also Débris-filled basins.

Valves in pumps........................................... W 14

Van Hise, C. R., Treatise on metamorphism...................... M 47

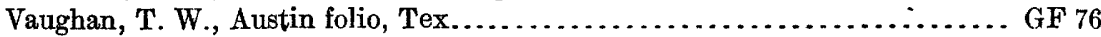

Geology and underground waters of the Edwards Plateau and Rio Grande

Plain, Tex...................................... 18 n b

Geology of the ground waters of Antigua; B. W. I.................. S 10

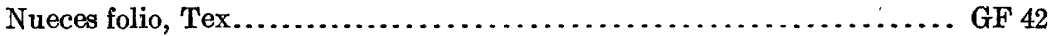

Rio Grande coal fields of Texas................................ B 164

Uvalde folio, Tex........................................... 64

Veatch, A. C., Coal and oil in Uinta County, Wyo................. B 285

Fluctuations of water levels in wells.......................... W 155

Geography and geology of southwestern Wyoming................... P 56

Geology and underground water resources of northern Louisiana and south-

ern Arkansas........................................ P 46

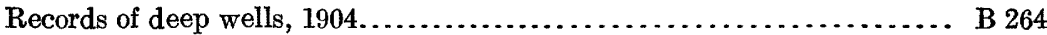

Underground water resources of Long Island, N. Y.................. P 44

Underground waters of Louisiana and southern Arkansas............... W 114

Veatch, J. O., Underground waters of the Coastal Plain of Georgia............. W 341

Vegetation, algae in ground water............................... W 338

as indicator of ground water.........................W 294, $375 \mathrm{~d}, 423$

carbonates in water in relation to............................. A $9 \mathrm{~d}$

crenothrix in ground water..................................... W 338

discharge of water by .................. $7,153,294,320,375 \mathrm{~d}, 423 ; \mathrm{S} 7$

ground water in relation to.... W $30,224,277,294,320,343,375 \mathrm{~d}, 380,423 ;$ B 308

hot springs in relation to.................................. $9 \mathrm{~d}$

quality of ground water in relation to . . . . . . . . . . . . . . . . . . W 343, 423

sulphuraria in ground water................................ W 338

water table in relation to............................ W $320,343,423$

zones of ground water in relation to............................. W 320

Veins, artesian water in.................................... B 319

Velocity of ground water. See Movements of ground water and Underflow measurements.

Vermont, areas, all of State............................... 102, 114

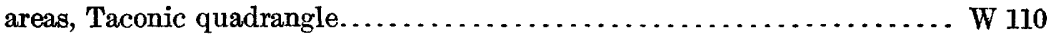

Ticonderoga quadrangle............................ W 110

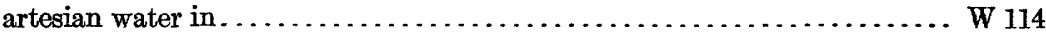

bibliography of ground water in ....................... W 114, 12n, 163

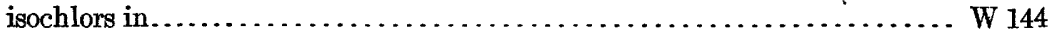

law relating to ground water in .................................. 152

mineral waters in......................... W 114; B 32; MR 1883-1916

public water supplies in ................................ W 102, 114

quality of ground water in . . . . . . . . . . . . . . . . 102,$114 ;$ B 32

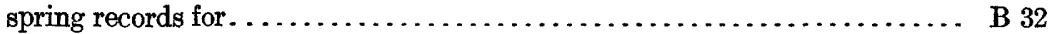

well records for . . . . . . . . . . . . . . . . . . . . . . . . $149 ;$ B 298

Vertical drainage. See Drainage into wells. 
Vesicles in rocks, artesian water in. . . . . . . . . . . . . . . . . . . $319 . \ldots$.

Vicksburg formation, water in, in Florida........................ W 319

water in, in Georgia. . . . . . . . . . . . . . . . . . . . . . . . . W 341; S 4

in Mississippi. ...................................... 159

Views. See Illustrations.

Virginia, areas, all of State. . . . . . . . . . . . . . . . . . . . . . 114

areas, Coastal Plain................................... 138; 8

Fredericksburg quadrangle. . . . . . . . . . . . . . . . . . GF 13

Manassas and vicinity . . . . . . . . . . . . . . . . . . . . . . 258

Nomini quadrangle.................................. GF 23

Norfolk quadrangle................................... 80

St. Marys quadrangle..................................... 136

Washington quadrangle....................................... 70

artesian water in ............................. 138; GF 23,70,80; S 8

bibliography of ground water in .................W 61, 114, 120, 149, 163

infiltration tunnels in ................................... S 8

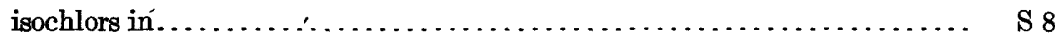

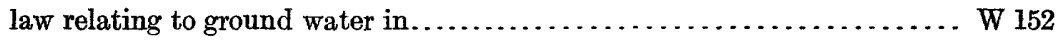

mineral waters in........................ B 32; MR 1883-1916; S 8

public water supplies in ............................ GF 80; S 8

quality of ground water in . . . . . . . . . . . W 258, 364; B 32; GF 80; S 8

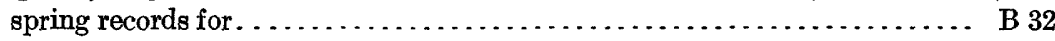

well records for................................. W 61,$149 ;$ B 298

wells in, construction of ...................................... S 8

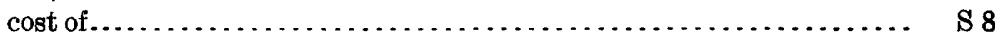

Viscosity of water, coefficient of.......................... $67 ;$ A 19 I b

Vitruvius on origin of ground water. . . . . . . . . . . . . . . .

Volcanic rocks. See Lava.

Volcanism, quality of ground water in relation to.............. B $330,491,616$

thermal springs in relation to................. $181 ; \mathrm{B} 330,491,616$

W.

Wall Creek sandstone, water in, in Wyoming.................. B 471 a

Waring, G. A., Geology and water resources of the Harney Basin region, Oreg.. W 231

Geology and water resources of south-central Oregon................W 220

Geology and water resources of south-central Washington............. W 316

Ground water in Reese River valley and adjacent areas, Nev ........ W $425 \mathrm{~d}$

Mineral springs of Alaska................................. W 418

Springs of California. . . . . . . . . . . . . . . . . . . . . . . . 338

Warm Springs. See Springs.

Warsaw formation, water in, in Missouri........................ W 195

Wasatch formation, water in, in Wyoming ...................... B 285

Washing down test pipe........................................... 141

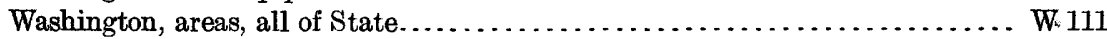

areas, Benton County....................................... B 298

Big Bend country............................... W 425 e

central...................................... W 425 e; B 108

Columbia River Plains. ............................ W 316

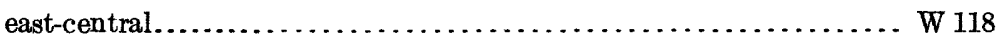

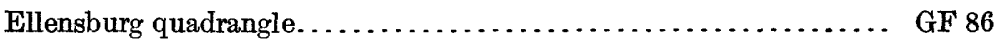

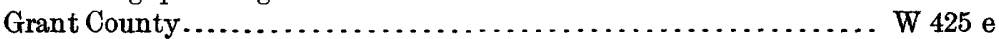

Horse Heaven Plateau................................ W 316

Jefferson County..................................... B 298

Mount Stuart quadrangle.............................. GF 106

Quincy Valley. . . . . . . . . . . . . . . . . . . . . . . . . . W 425 e 


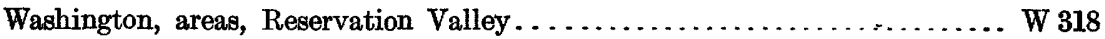

south-central. . . . . . . . . . . . . . . . 316

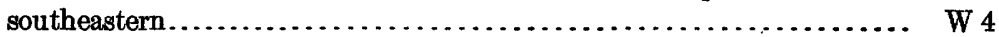

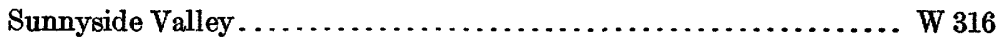

Walla Walla County.................................. B 298

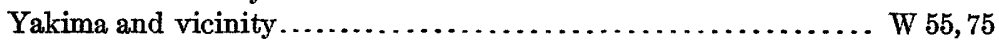

artesian water in................... W 4, 55, 75, 118, 316; B 108; GF 86, 100

bibliography of ground water in......................... W 120, 163

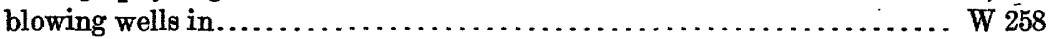

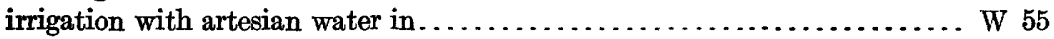

with ground water in .................... W 55, 316, 425 e; A 16 II $\mathrm{e}$

laws relating to ground water in $\ldots \ldots \ldots \ldots \ldots \ldots \ldots \ldots \ldots \ldots \ldots \ldots \ldots \ldots \ldots \ldots, 78,122,152$

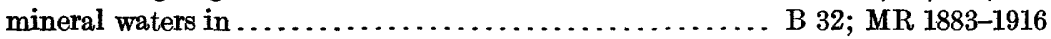

public water supplies in ................................ W 111

quality of ground water in ......................... 111, $425 \mathrm{e} ; \mathrm{B} 32$

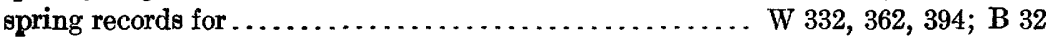

stock-watering places in .................................. W 316

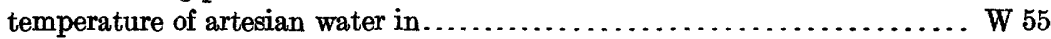

well records for............................ W 61, 149; B 264, 298

Washington limestone, water in, in Pennsylvania .................. G F 121

Washita formation, water in, in Texas.......................... W 276

Waste-filled basins. See Débris-filled basins.

Waste of ground water. See Conservation.

Water in mines. See Analyses of mine waters, Mines, and Quality of mine waters.

Water-bearing formations. See specific formations; also under Maps.

Water-bearing material, effective size of ................... W 67; 140; P 44 uniformity coefficient of ................................ P 44

"Water catches" for collecting rain water, construction of ................ W 380

"Water finders" ........................................... W 416

Water horizons. See specific formations.

Water rights. See Law relating to ground water.

Water spreading in California.................................... S 6

Water-stage recorders used in wells ...................... W 155; A 19 II b

Water supplies. See Irrigation, Public water supplies, and Watering places on routes of travel.

Water table, definition of ..................................... W 67

fluctuations of ..... W $10,12,18,29,30,58,67,137,138,139,142,153,155,164$, $213,219,232,251,256,258,294,319,320,331,343,345 \mathrm{~g}, 345 \mathrm{~h}, 375$ a, 400 e, 423; A 19 Ir b, 21 rv a; M 47; P 44; G F 120, 193; S 6, 8, 9.

air in soil in relation to ............................ $19 \mathrm{II} b$ barometric pressure in relation to ....... W 155, 258; $\AA 19$ II b; M 47; P 44 capillarity in relation to................................ W 155 causes of...................................... W 155; M 47

- dams in relation to.................................. W 155; P 44

deforestation in relation to.................................. W 155

denudation and valley filling in relation to..................... M 47

discharge of ground water in relation to ................. W 155, 294

evaporation in relation to........................... W 294, 423

geologic causes of....................................... W 155

human agencies in relation to.......................... W $155 ;$ M 47

irrigation in relation to...................... W 142, 155; $219,345 \mathrm{~h}$

methods of determining................................... 155

plastic formations in relation to .......................... 155

$56122^{\circ}-18-$ WSP $427-11$ 
Water table, fluctuations of, precipitation in relation to

principles of. W 155; B 529 pumping in relation to W 142, 155, 219, $345 \mathrm{~h} ; \mathbf{P} 44$ quantity of ground water indicated by ............... W $345 \mathrm{~h}, 400 \mathrm{e}$ railroad trains in relation to................................. 155 settlement in relation to.................................. W 155 streams in relation to..................................... 155 temperature in relation to....................... W 155; M 47; P. 44 tides in relation to......................W 155, 232; P 44; GF 193 uplift and subsidence in relation to ........................M 47 in Arizona . W 104, 136, 320, 375 b, 425 a; GF 129 in Arkansas........................................ $399 ;$ P 46 in California . . .............................. $58,137,138,139$, $142,146,213,219,222,225,251,331,345 \mathrm{~h}, 375$ a, 398, 400 e; S 6,7 in Colorado. W 9; P 94; B 265; GF 120 in Connecticut.............................. $160,232,374,397$ in crystalline rocks...................................... W 232

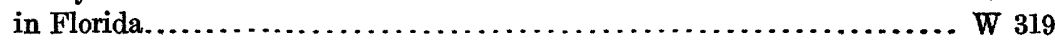
in Great Plains............................ $21 \mathrm{rv}$ c, $22 \mathrm{rv} \mathrm{c}$

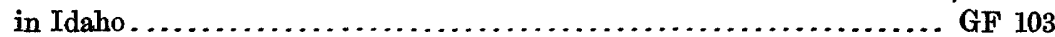
in Illinois.................................. 506 ; GF 67; 3

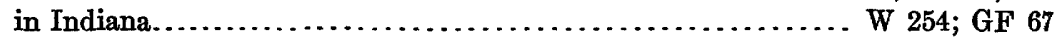
in Kansas..................................... 6, 153, 258 in Kentucky..................................... 164, 233 in Louisiana ........................... P 46 in Michigan................................. 182, 183; GF 155

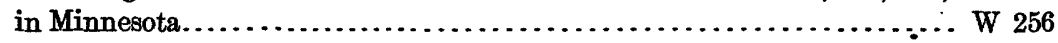
in Mississippi.............................................. 159 in Montana...................................... $345 \mathrm{~g}$ in Nebraska........................ W 12, 29, 184, 425 b; A 21 rv a in Nevada................................... W 365, 423, $425 \mathrm{~d}$ in New Mexico..........W 10, 188, 275, 343, 345 c, 422, 425 a; B 618; GF 207 in New York ......................................... P 44 in Oregon.......................................... 220, 231; GF 103 in Pennsylvania.......................................... 300 in Tennessee . ............................................ 164

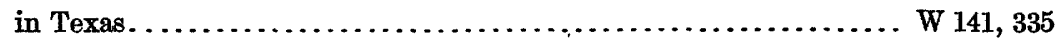
in Utah. ............................. 157, 199, 217, 277, 333 in Virginia . . . . . . . . . . . . . . . . . . . . 8 in Washington......................................... $425 \mathrm{e}$

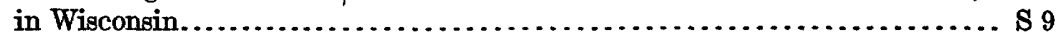
in Wyoming. . . . . . . . . . . . . . . . . . 425 b perched.........................W 164, 233, 258, 320, 335; P 44, 46; S 8 relation of artesian head to.....................W 256, 293; A 5 c; S 9 discharge of ground water to..................W 155, 294, 320, 423; S 7 faults to........................................W $345 \mathrm{~h} ; 423$ oil and gas to............................................ 658 ore deposits to.............. M 47; P 94; B 529, 625; GF 112, 120, 129 sulphides to..................................... B 529, 625 topography to....................... W 67, 159, 254, 374; A 19 m b vegetation to............................. W 294, 320, 343, 423 shape of..................................... $67 ;$ A 19 In b See also Maps. 
Water wheels for lifting water.

W $1,4,13,29$

Water witching, bibliography of

fallacy of

W 255, 278, 416; MR 1882 a

history of.

W 416; MR 1882 a

Watering places on routes of travel in Arizona

W 380; B 352

in California

W 224; B 308

in Nevada.

W 224, 365; B 308

in New Mexico....................................... W 343, 380

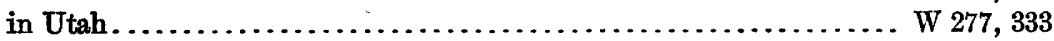

"Waterlime" formation, water in, in Indiana..................... W 114, 254

Water works. See Public water supplies.

Waverly shale, water in, in Indiana............................ W 114

water in, in Kentucky...................................... 233

Waynesburg sandstone, water in, in Pennsylvania.................... GF 121

Weather, effects of, on wells. See under Water table and Wells.

Weathered rocks, water in.................................. W 114

Weathering produced by ground water.......................... 47

Weed, W. H., Fort Benton folio, Mont........................... GF 55

Hot springs of southern United States. . . . . . . . . . . . . . . . W 145

Little Belt Mountains folio, Mont........................... GF 56

Travertine and siliceous sinter of hot springs................... 9 d

Weeks, F. D., Underground waters of New York. . . . . . . . . . . . W 114

Well and spring records for New York....................... W 102

Wegemann, C. H., Oil and gas in Palo Pinto County, Tex.............. B 621 e

Powder River oil field, Wyo.............................. B 471 a

Weidman, Samuel, Water supplies of Wisconsin..................... S 9

Weirs, coefficients, formulas, and tables relating to............... 150,200

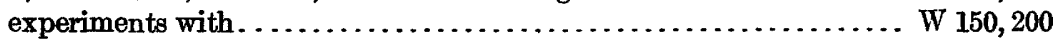

portable.................................................. 320

Welitschkowsky, on flow of water and air through soils............. A 19 II a

Well casings............................................. 145

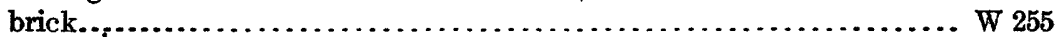

cement........................................... 255,293

corrosion of. .............................................. 293

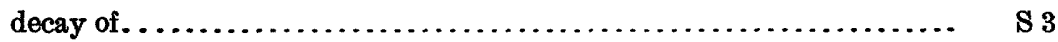

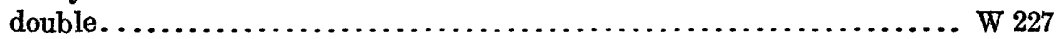

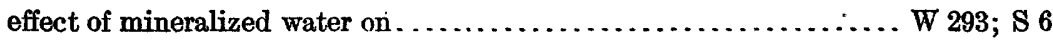

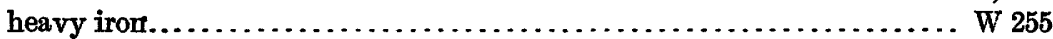

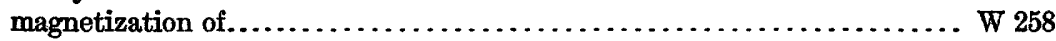

methods of inserting........................... $223,257,293$

perforation of.................................. 110, 140, 257

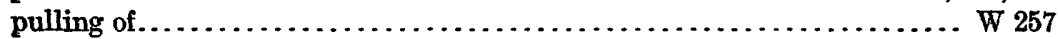

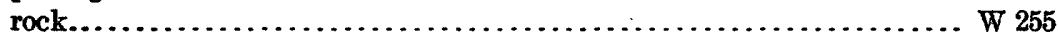

sheet iron. . . . . . . . . . . . . . . . . . 255

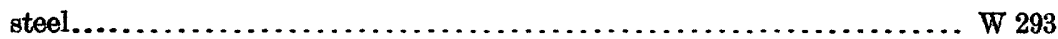

stovepipe............................... 110, 140, 255, 257

straightening of . . . . . . . . . . . . . . . . 257

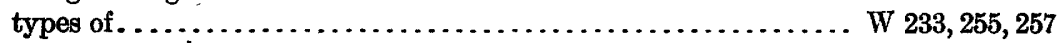

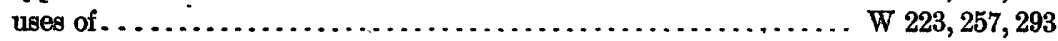

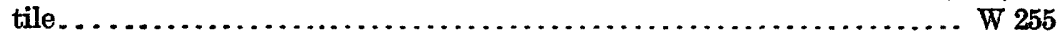

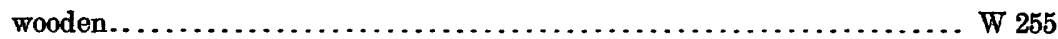

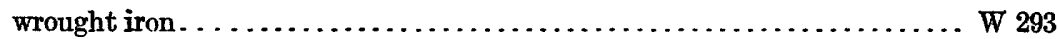

Welliconstruction, accidents in.......................... W 257, 293

artesian ....................... W 118,227, 257; A 5 c, 17 I g; $\mathbf{S} 9$

augers for. . . . . . . . . . . . . . 
Well construction, boring method of W 255; $\mathrm{S} 8$

boulders interfering with W 257

"California" method of. W $110,140,220,231,255,257$ calyx drills for. W 255, 257 chilled-shot drills for. W 257 churn drills for W 232, 257 controlling flows in. W 118 ; A 5 c core drills for. W 257; P 46 crooked holes in. W $256,257,293$ deep-drilling methods of .............................. W 257, 293 detecting flows in: W 118, A 5 c

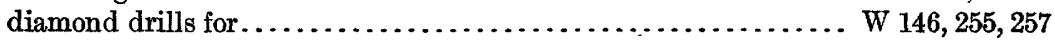

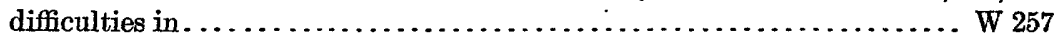
digging method of .................................. 232,254 double casing used in . . . . . . . . . . . . . . . . . . . . . . . 227 driving method of ........ W 140, 145, 232, 254, 255, 257; A 19 II b; P 46; S 8 explosives used in W $223,255,257$ finishing methods of. W 255, 256, 293, 343; P 46 for drainage purposes. W 258 for underflow measurements. W 112, 140 friction in W 257 gravel screens developed in $345 \mathrm{~g}$ history of. W 257 hollow-rod method of W 257 hydraulic method of. $\mathrm{W} 255,256,257 ; \mathrm{P} 90 \mathrm{~h}$ in Arizona. W 380 in Arkansas. $\mathrm{W} 145 ; \mathrm{P} 46$ in California W $52,110,140,257,375$ a in Colorado. W 240 ; GF 68 in Connecticut................................... W 232, 374, 397 in Great Plains. ............................................ 22 rv c

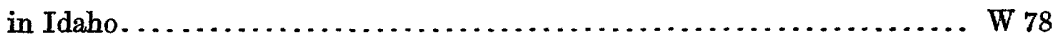

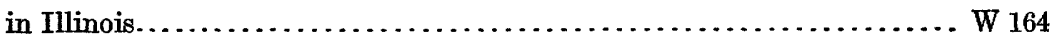
in Indiana. . . . . . . . . . . . . . . . . . . . . . . . . . . . . . . . . . 254 in Iowa . . . . . . . . . . . . . . . . . . . . . 293

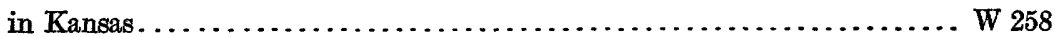
in Kentucky ............................................... 164 in Louisiana. . . . . . . . . . . . . . . . . . . . . . . . . . . 46

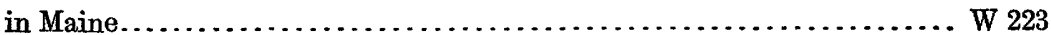
in Michigan . ...................................... W 30, 182, 183 in Minnesota................................................. 256 in Montana...................................... W $375 \mathrm{~g}, 400 \mathrm{~b}$ in Nebraska.................................... 184, 215, 258 in Nevada....................................... W $375 \mathrm{~d}, 423$ in New Mexico. ......................................... W 343 in Oregon........................................ 78,231 in South Dakota........................ W 34, 227; A 17 II g; GF 96 in Tennessee............................................ 164

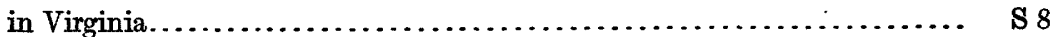

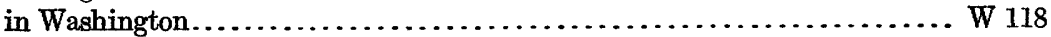

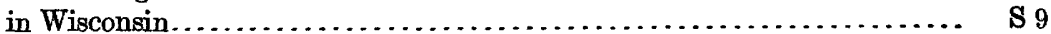
jetting method of ..............W 101, 164, 231, 254, 255, 256, 257; P 46; S 8 joints in rock in relation to.............................. 223,256 
Well construction, loss of tools in.............................. W 257

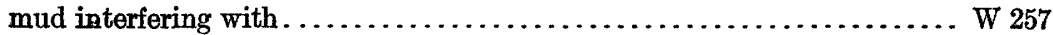
oil-well rigs used in............................. W 257; B 282 oil-well, to exclude water from. . . . . . . . . . . . . . . . . . . . . . . . B 658 on alkali flats....................................... B 530 r packers used in . ................. W 54, 118, 160, 257, 293; A 5 c; S 9 percussion drills for..................................... 257 ; 8

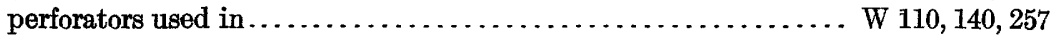

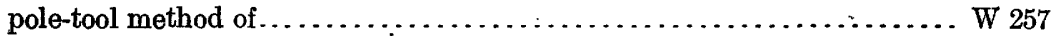

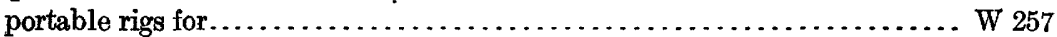
pumping method of ............................... 255, 343, $345 \mathrm{~g}$ punching method of . ............................. 255, 257; P 46 quicksand interfering with.................... W 30, 257,375 d, 423

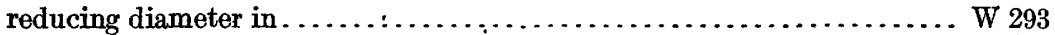
rotary drills for..................... $101,232,255,257 ; \mathrm{P} \mathrm{46,90} \mathrm{h} ; \mathrm{S} 8$ "sand bucket" method of ................................W 231, 258 sand pumping in connection with............ W 231, 256, 258, 293, 343; P 46 seed-bag packers used in . ........................ W 54, 118; A 5 c self-cleaning drills for...................................... 257

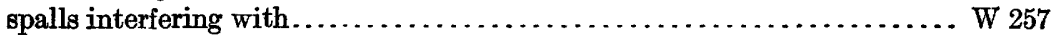

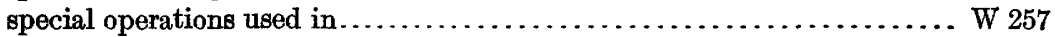

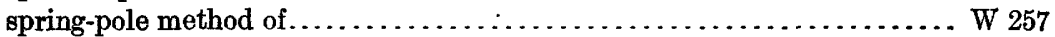

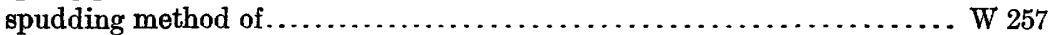

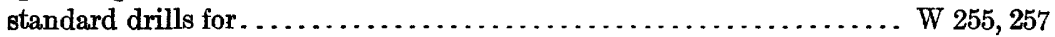
steel-shot drills for.................................. 255, 257 stovepipe method of ..................... W 110, 140, 220, 231, 255, 257

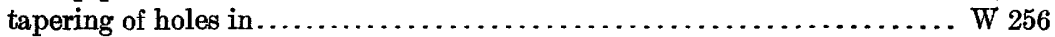
time required for........................................ 68

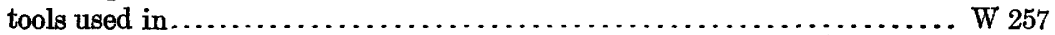

Well data, symbols for, on maps.................................. 160 Well drilling. See Well construction.

Well drillings, fossils in ................................ $293 ; \mathrm{P} 90 \mathrm{~h}$ interpretation of ................................ $293 ; \mathrm{P} 90 \mathrm{~h} ; \mathrm{S} 9$ methods of collecting.................... B 264,298; P $90 \mathrm{~h} ; \mathrm{S} 9$ methods of examining............................ W $293 ; \mathrm{P} 90 \mathrm{~h}$

Well drills, manufacturers of............................... W 257

Well packers.......................... W $54,118,160,257,293 ;$ A 5 c; S 9

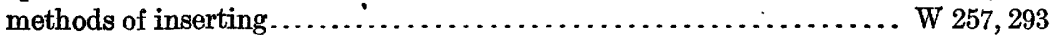

Well records, correlation by means of .....................W $293 ; \mathbf{P ~} 90 \mathrm{~h}$ for Alabama...................... $57,102,149 ;$ B 264, 298; S 2 for Arizona. W 57, 104, 136, 149, 320, $375 \mathrm{~b}, 380$; B 298, 352, 435, 540 p; GF 111, 112 for Arkansas.....................W 57, 102, 145, 149, 399; B 264, 298; P 46 for California............................................ 45 , $57,58,60,89,137,138,139,142,149,181,213,219,222$, $225,251,278,294,331,345 \mathrm{~h}, 398$; B 264, 298, 540n; S 7 for Colorado................................ W 57, 149, 240, 264; B 298; A 16 II f, 17 II f; M 27; P 32, 52; B 131; GF 135, 186 for Connecticut................ W 57, 102, 110, 149, 232, 374, 397; B 264, 298 for Delaware....................W 57, 114, 149; B 138, 298; GF 137, 162 for District of Columbia................ W 57, 114, 149; B 138; GF 70, 152 for Florida........................... $57,102,149,319 ; \mathrm{B} 264,298$ for Georgia.....................W 57, 67, 102, 149, 341; B 138, 264, 298; S 4 for Hawaii................................ W 318, 336, 373,430; S 12 
Well records; for Idaho .................. W 57, 149; B 199, 264, 298; GF 45, 104 for Illinois.

149, 164; B 264, 298, 438, 506; A 17 I h; M 38; GF 67, 81, 105; S 3 for Indiana........ W 21, 26, 113, 149, 254; B 264, 298; A $18 \mathrm{Iv} \mathrm{b;} \mathrm{GF} \mathrm{67,} \mathrm{84,} 105$ for Iowa ............................W 57, 149, 293; B 264, 298; GF 156 for Kansas......... W 57, 149, 153, 258, 273, 345 a; A 16 In f; P 32; B 131, 264, 298 for Kentucky ..................W 57, 102, 149, 164, 233; B 264, 298; GF 184 for Louisiana........................ W 101, 149; P 46; B 264, 298 for Maine....................... W 57, 102, 145, 149, 223; B 264, 298 for Maryland...................... W 114, 149; B 138, 298; GF 70, 152 for Massachusetts....................... W 57, 102, 110, 149; B 264, 298 for Michigan....................... W 57, 102, 149, 160, 182, 183; B 264, 298 for Minnesota............ W 57, 102, 149, 193, 256; M 25; B 264, 298; GF 117, 201 for Mississippi.......................W 102, 149, 159; P 46; B 264, 298 for Missouri..........................W 57, 102, 149, 195; B 264, 298, 438 for Montana.......................W 57, 149, 221, $345 \mathrm{~g}, 400 \mathrm{~b} ;$ B 264, 298 for Nebraska...................................... W 12, 29, $61,149,215,216,425$ b; A 16 II f; P 32; B 131, 264, 298; GF 88, 108, 156 for Nevada...................... W 61, 149, 365, 423; P 95 a; B 530 r, $540 \mathrm{n}$ for New Hampshire.......................... W 61, 102, 149; B 264, 298 for New Jersey ...........W 61, 106, 114, 149; B 138, 264, 298; GF 157,161, 162 for New Mexico....... W 61, 149, 158; 188, 275, 343, 345 c, 380; B 264, 298, 435, 618 for New York............. W 61, 102, 110, 149; P 44; B 138, 264, 298; GF 157 for North Carolina.............W 61, 114, 149; B 138, 264, 298; GF 80, 124; S 5 for North Dakota.......... W 61, 149; A 17 II g; M 25; B 298; GF 117, 168, 181 for Ohio................ W 61, 149,259; A $18 \mathrm{rv} \mathrm{b}, 19 \mathrm{rv} \mathrm{b;} \mathrm{B} \mathrm{264,} \mathrm{298;} \mathrm{GF} 184$ for Oklahoma..................... W 57, 61, 148, 149, $345 \mathrm{~b}, \mathrm{~d}$; B 264, 298 for Oregon........................W 61, 149, 220, 231; B 252, 264, 298 for Pennsylvania.......... W 61, 106, 149; B 138; 264, 298, $531 \mathrm{~d}$; GF 121, 123, 162 for Rhode Island ...........................W 61, 102, 149; B 264, 298 for South Carolina....................W 61, 114, 149; P $90 \mathrm{~h} ; \mathrm{B} 138,298$ for South Dakota ............. W 34, 61, 90, 149, 227; A 17 In g, 18 rv c, 21 iv b; P 32, 65; B 264, 298; GF 96, 99, 100, 107, 108, 113, 114, 156, 164, 165 for Tennessee....................W 61, 102, 149, 164; B 264, 298; GF 124 for Texas.................................W 13, 61, 149, 190, 276, $317,335,343,375 \mathrm{~g}$; A 18 II b, 21 vm; P 46; B 264, 298; GF 166; S 1 for Utah............... W 61, 149, 157, 199, 217, 277, 333, 380; B 264, 298, 541 d for Vermont................................W 102, 110, 149; B 298 for Virginia....................W 61, 114, 149,258; B 138, 298; GF 70,80; S 8 for Washington.............W 55, 61, 111, 118, 149, 316; B 264, 298; GF 86, 106 for West Virginia........................ W 61, 149; B 264, 298; GF 184 for Wisconsin.....................W 61, 149; B 264, 298; GF 140; S 9 for Wyoming... W 61, 149; A $21 \mathrm{rv} \mathrm{b;} \mathrm{P} \mathrm{32,} \mathrm{56,} \mathrm{65;} \mathrm{B} \mathrm{298,} \mathrm{364,} \mathrm{621} \mathrm{1;} \mathrm{GF,} \mathrm{107,} 173$ forecasting ground water by means of............................ 293 importance of......................................... B 264 methods of making and obtaining........................ W 293; B 264 preservation of....................................... 78, 256 See also other papers listed under specific States, many of which also contain well records.

Well screens, construction of ................................. W 101 efficiency of.......................................... 110,375 a gravel............................ W 255, 256, 293, 343, $345 \mathrm{a}, \mathrm{g}, 375 \mathrm{a}$ incrustation of. W 256, 293 
Well screens, load produced by clogging of..................... W 9

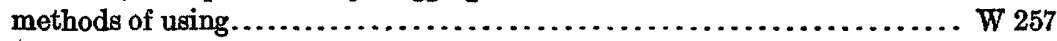
natural............................... W 256, 293, 343, $345 \mathrm{~g}, 375$ a

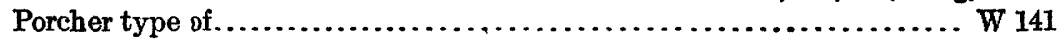

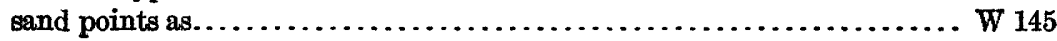
types of............................. 71, 255, 257, 375 a; P 46

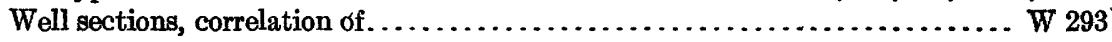

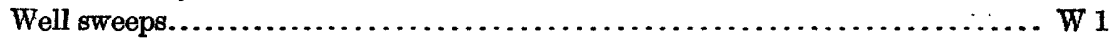

Wells, barometric effects on ................................. W 29,

$155,195,215,216,256,258,317 ;$ A 19 II b; M 47; P 44; B 19; GF 156 barometric effects on, bibliography of....................... 155

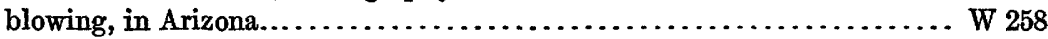

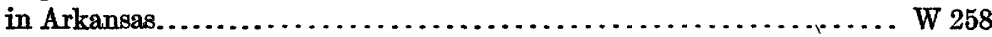

in Colorado......................................... 16 unf

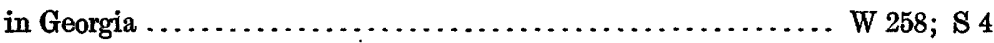

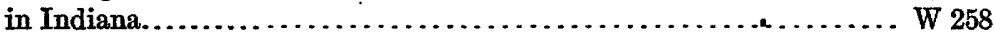

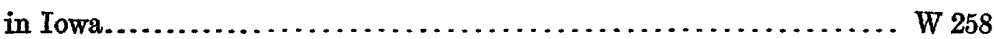

in Kansas.................................... 16 If

in Louisiana........................................ 101,258

in Michigan..................................... 30, 258; GF 205

in Minnesota................................... 256, 258

in Missouri....................................... 195, 258

in Nebraska......................... 29, 215, 216, 258; A 16 пा f

in New York..................................... W 258; P 44

in Oregon ............................................... 258

in South Carolina........................................ 258

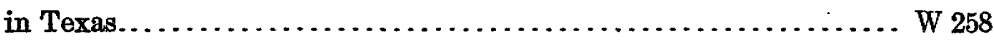

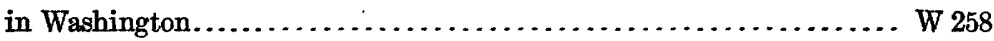

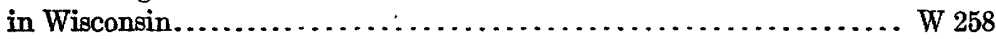

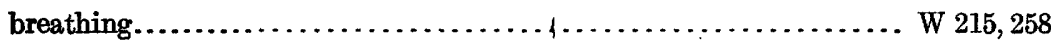

See also Wells, barometric effects on, and Wells, blowing.

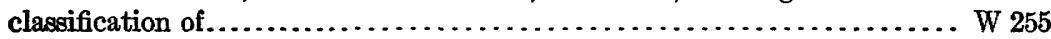

cleaning of..................................... $67,255,257$

to develop gravel screens....................W 256, 293, 343, 345 a, g

clogging of.................................. $8,14,256,257,293$

combined with cisterns....................................... 255

connected......................................... W 223, 293

cost of........................................... 110, 255, 257

drilled by jetting process........................... W 255, 277

drilled in crystalline rocks.......................... W 160,258

drilled in shale........................................ W 258

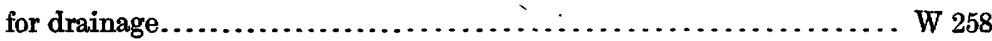

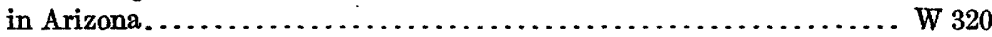

in Arkansas........................................ $399 ; \mathrm{P} 46$

in Atlantic Coastal Plain................................ W 258

in California............ W 137, 138, 139, 142, 219, 222, 225, 278, 375a, 398

in Colorado ........................... 9, 240; B 131; GF 68

in Connecticut...................................... 160

in Great Plains................................... 11 rr c

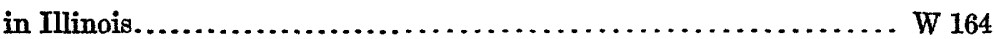

in Kentucky.................................. W 164, 233

in Kansas.............................................. B 131

in Louisiana................................... B 282; P 46 
Wells, cost of, in Maine................................ 223, 258

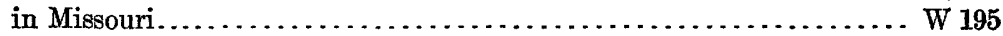

in Montana........................................... 400

in Nebraska .................................. W 29; B 131

in Nevada.......................................... 423

in New Mexico.................................. W 10,345 c

in Oregon ...................................... W 220, 231

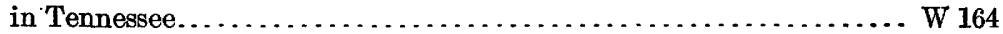

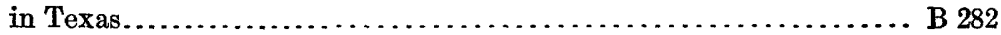

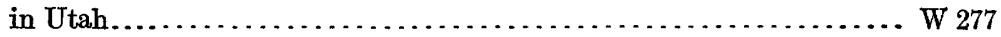

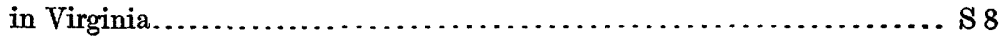

deep, bibliography of .............................. 57,61

list of.................................. $57,61,149,257$

diameters of, yield and efficiency in relation to...................W 293

drainage into............................... W 145, 160, 256, 258, 293

explosives used in................................ W 223, 255, 257

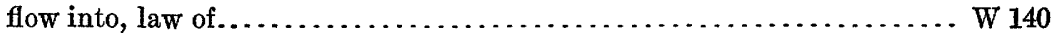

flow of, methods of increasing........................... W 118; A 5 c

flows in, method of detecting.......................... 118; A 5 c

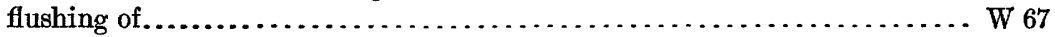

for underflow measurements........................... W 112, 140, 141

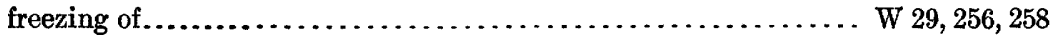

horizontal type of. See Infiltration ditches and tunnels.

hot water in. See under Temperature.

improvement of water in................................... W 160 interference of .............W 67,122, 184, 223; A 19 Ir b;M 27; GF 97; S 8, 9

law relating to..................................... 122

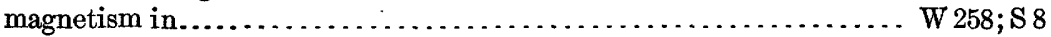

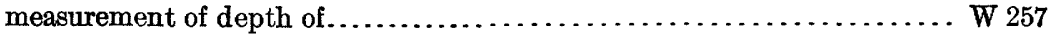

natural, caused by sink holes ........................ A 21 rv c

packing of ....................... $54,118,160,257,293 ;$ A 5 c; S 9

pollution of. See under Pollution.

rise of water in, methods of measuring rate of ......... W 140, 155; A $19 \mathrm{~m} \mathrm{~b}$

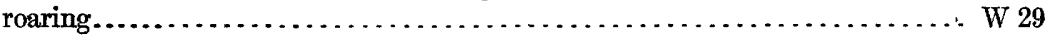

screening of. See Well screens.

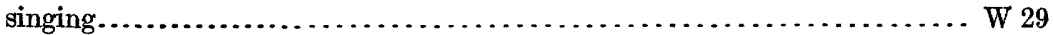

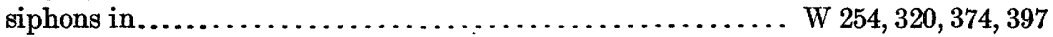

specific capacity of ............W 140, 141, 153, 258, $400 \mathrm{~b}, 425 \mathrm{~b} ; \mathrm{P} 44$; S 11

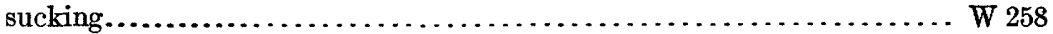

sunk by States. . . . . . . . . . . . . . . . . . . . . . . . . . . . . . W 122

testing of, deceptions in ....................................... 257

water levels in, methods of obtaining continuous records of... W W 155; A 19 II b weather................................................ 215

yield of, depth in relation to........................... W 160, 232

diameter in relation to . . . . . . . . . . . . . . . . . . . . . . W 293

fluctuations in . . . . . . . . . . . . . . . . . . . . . . . . 256,258

methods for measuring............W $67,110,150,157,200,219,240,320$

methods of increasing . . . . . . . . . . . . . . . . . . W 255, 257, 343

principles of........................ W 67, 140, 293; A 19 Ir b

temperature of water in relation to ................. W $400 \mathrm{~b} ; \mathrm{A} 5 \mathrm{c}$

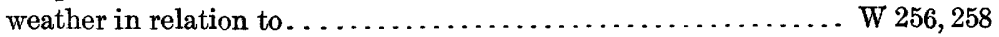

See also Artesian water, Artesian wells, Well casings, Well construction, and

Well screens. 
Wesbrook, F. F., Quality of surface waters in Minnesota.............. W 193

West Indies, areas, Antigua ..................................... 10

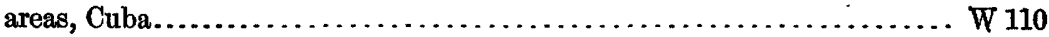

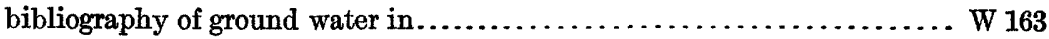

West Virginia, areas, Accident quadrangle....................... GF 160

areas, all of State. . . . . . . . . . . . . . . . . . . . 114

Cabell County..................................... 298

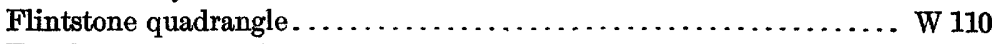

Frostburg quadrangle.................................... 110

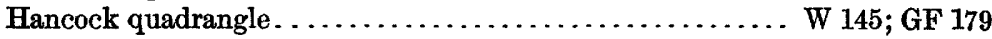

Kenova quadrangle.................................. GF 184

Nicholas quadrangle ................................ W 145

Pawpaw quadrangle............................ W 145; GF 179

artesian water in .................................... 160,179

bibliography of ground water in...............W 61, 114, 120, 149, 163

law relating to ground water in ................................. 152

mineral waters in........................ W 114; B 32; MR 1883-1916

quality of ground water in ............... W 145, 364; B 32; GF 179, 184

salt water in .................................. 530 b; GF 184

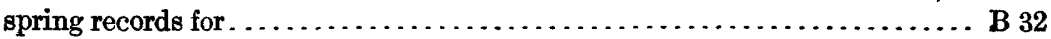

well records for............................. W 61 149; B 264, 298

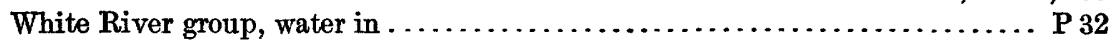

Whitfield, J. E., Analyses of waters of Yellowstone National Park.......... B 47

Whitney, F. L., Artesian water at Ithaca, N. Y.................. W 110

Wichita formation, water in, in Texas......................... W 317

Wicomico formation, water in, in Delaware..................... GF 137

water in, in Maryland.................................... GF 137

Wilcox formation, water in, in Arkansas. . . . . . . . . . . . . . . . . W 399

water in, in Georgia....................................... 341

in Mississippi....................................... 159

in Texas........................................... 276,335

See also Sabine formation.

Willard, D. E., Casselton-Fargo folio, N. Dak.-Minn................ GF 117

Jamestown-Tower folio, N. Dak........................ GF 168

Williams, H. S., Eastport folio, Me........................... GF 192

Watkins Glen-Catatonk folio, New York. . . . . . . . . . . . . . . . GF 169

Willis, Bailey, New York City folio ........................... GF 83

Wilson, H. M., American irrigation engineering................ A $13 \mathrm{~mm} \mathrm{~b}$

Pumping for irrigation..$\ldots \ldots \ldots \ldots \ldots \ldots \ldots \ldots \ldots \ldots \ldots \ldots \ldots \ldots \ldots \ldots \ldots \ldots \ldots$

Winchester, D. E., Lignite field of northwestern South Dakota.......... B 627

Winchester limestone, water in, in Kentucky .................... W 233

Windmills, "angle of weather" in................................ W 20

battle-ax type of . . . . . . . . . . . . . . . . . . . . 29

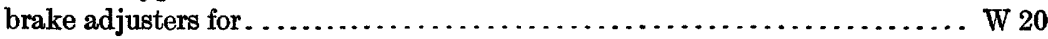

classification of . . . . . . . . . . . . . . . . 41

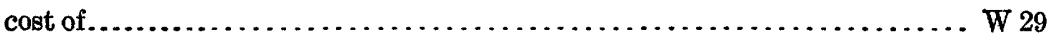

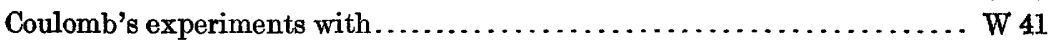

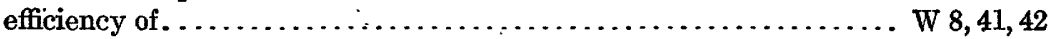

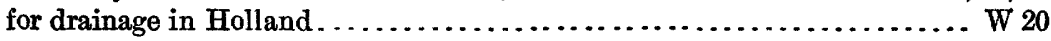

experiments with. ................................ $20,41,42$

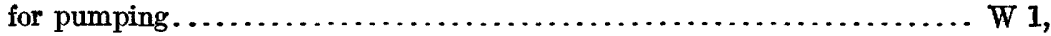

$8,12,13,14,20,29,41,42,260,275,343 ;$ A 19 Iv c, 22 Iv c; P $17 ;$ GF 103

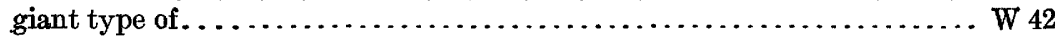

Griffith's experiments with . ................................. 41 


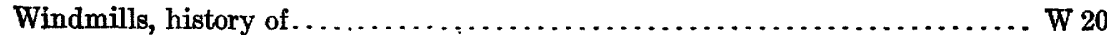

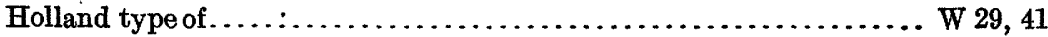

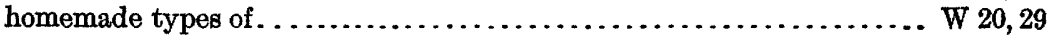

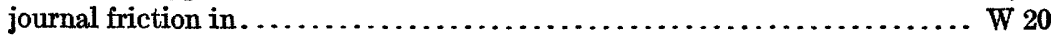

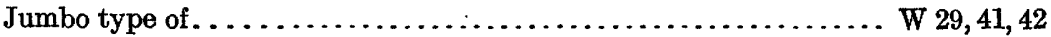

King's experiments with $\ldots \ldots \ldots \ldots \ldots \ldots \ldots \ldots \ldots \ldots \ldots \ldots \ldots \ldots, \mathrm{W} 41$

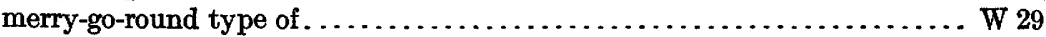

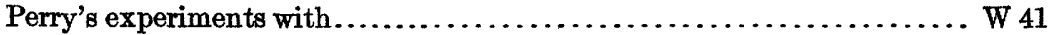

regulating devices for $\ldots \ldots \ldots \ldots \ldots \ldots \ldots \ldots \ldots \ldots \ldots \ldots \ldots \ldots \ldots \ldots \ldots, W(1$

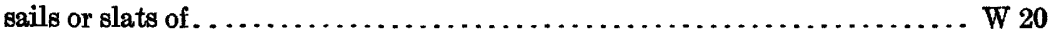

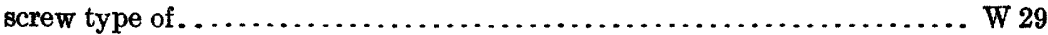

Smeaton's experiments with $\ldots \ldots \ldots \ldots \ldots \ldots \ldots \ldots \ldots \ldots \ldots \ldots \ldots \ldots \ldots \ldots \ldots, W(1$

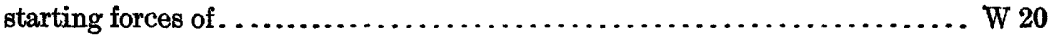

testing, apparatus for..................................... W 20,41,42

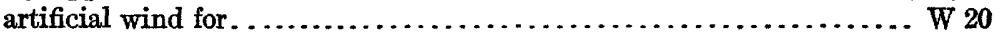

methods of $. \ldots \ldots \ldots \ldots \ldots \ldots \ldots \ldots \ldots \ldots \ldots \ldots \ldots \ldots \ldots \ldots \ldots \ldots \ldots \ldots, 2,4,41,42$

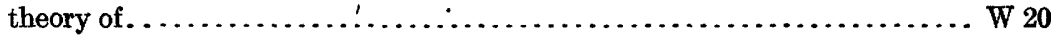

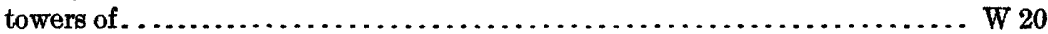

Winslow formation, water in, in Oklahoma................... GF 132, 154

Wisconsin, areas, all of State............................. W 114; 9

areas, Lancaster quadrangle.............................. GF 145

Manitowoc County ................................... B 298

Milwaukee quadrangle.............................. GF 140

Mineral Point quadrangle....................... W 145; GF 145

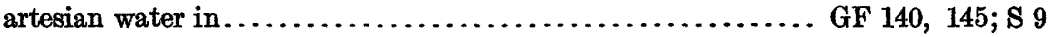

bibliography of ground water in................ W $61,114,120,149,163$

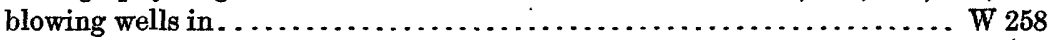

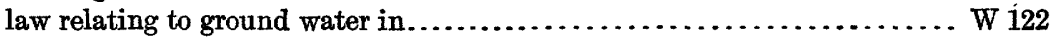
mineral waters in .............. W 114; B 32; MR 1883-1916; GF 140; S 9 public water supplies in............................... GF 140; 89 quality of ground water in...................... B 32; GF 140; S 9

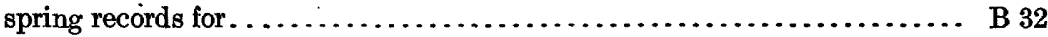

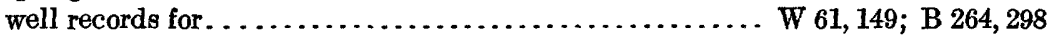

Wissahickon gneiss, water in, in Pennsylvania....................... W 106 "Witching" for water. See Water witching.

Wolff, H. C., Underflow near St. Francis, Kans.................... W 258

Underflow of South Platte Valley............................. W 184

Wolff, J. E., Franklin Furnace folio, N. J........................ GF 161

Wollug on flow of water and air through soils....................... A 19 Ir b

Wood, B. D., Publications of U. S. Geological Survey relating to water re-

sources............................................... W 340

Woodbine sand, water in, in Texas........................ W 276; A 21 vIr

Woodruff, E. G., Geology and petroleum resources of the De Beque oil fields,

Colo ................................................ B $530 \mathrm{c}$

Woolen mills, quality of water for.............................. W 398

Woolsey, L. H., Bull Mountain coal field, Mont..................... B 647

Wyoming, areas, Aladdin quadrangle......................... GF 128

areas, Bald Mountain quadrangle........................... GF 141

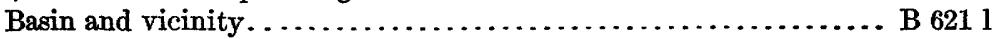

Bighorn Basin................................. P 53; B 656

Big Horn County ..................................... B 6211

Bighorn Mountains.................................. P 51

Black Hills region......................... A 21 Iv b; P 65 
Wyoming, areas, central ......................................... 641 i

Cloud Peak quadrangle............................... GF 142

Dayton quadrangle.................................... GF 141

Devils Tower quadrangle.............................. GF 150

eastern................................. A 21 rv c, 22 rv c; P 32

Fort McKinney quadrangle........................... GF 142

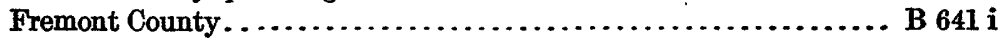

Goshen Hole quadrangle................................ W 70

Laramie Basin.................................... B 364

Laramie quadrangle.................................. GF 173

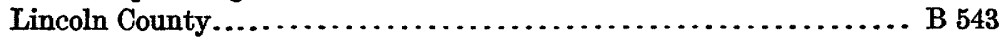

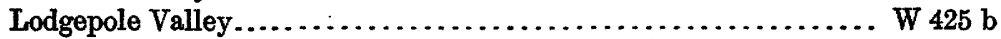

Natrona County $\ldots \ldots \ldots \ldots \ldots \ldots \ldots \ldots \ldots \ldots \ldots \ldots \ldots \ldots \ldots \ldots \ldots$, B 641 i

Newcastle quadrangle............................... GF 107

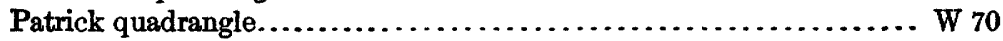

Powder. River oil field................................ B 471 a

Sherman quadrangle.................................. GF 173

southeastern..$\ldots \ldots \ldots \ldots \ldots \ldots \ldots \ldots \ldots \ldots \ldots \ldots \ldots \ldots \ldots \ldots \ldots$, B 364

southwestern $\ldots \ldots \ldots \ldots \ldots \ldots \ldots \ldots \ldots \ldots \ldots \ldots \ldots \ldots \ldots \ldots \ldots \ldots \ldots, \quad$ P 53

Sundance quadrangle. ............................... GF 127

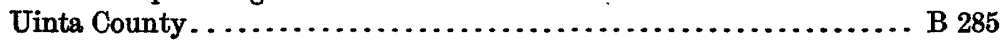

Yellowstone National Park............... W 364; A 9 d, 14 II b; B 47, 395 artesian waterin.................... W 425 b; A 21 rv b; P 32, 51, 53, 65; B 285, 364, 543, 641 i; GF 107, 127, 128, 141, 142, 150, 173 bibliography of ground water in ...................... W 61, 120, 149,163 irrigation with ground water in................ W $425 \mathrm{~b} ; \mathrm{A} 21 \mathrm{rv}$ c, $22 \mathrm{IV}$ c

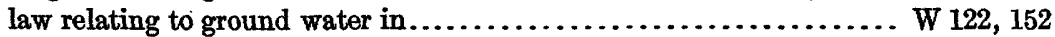

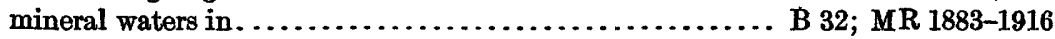
quality of ground water in.............. W 364, $425 \mathrm{~b} ; \mathrm{A} 9 \mathrm{~d}, 21 \mathrm{rv}$ b; P 32; B 32, 285, 364, 471 a, 641 i; GF 107, 173 spring records for. . . . . . . . . . . . . . . . 32 well records for............................... W 61, 149; B 298 See also Yellowstone National Park.

Y.

Yakima basalt, water in, in Washington. See under Lava.

Yegua formation, water in, in Texas.......................W 335, $\mathbf{3 7 5} \mathrm{g}$ Yellowstone National Park, quality of water in............ W 364; A 9 d; B 32, 47 radioactive waters in.............................. B 395; MR 1913 In $\mathrm{h}$ springs and geysers in.................... W 364; A 9 d, 14 II b; B 47, 395

Yield of wells. See under Wells.

Yorktown formation, water in, in North Carolina ...................... \& 5 water in, in Virginia........................................... 8 See also Chesapeake group.

$\mathrm{Z}$.

Zigzag balances for lifting water.

Zone of flowage, ground water in relation to........................ M 47 Zone of fracture, ground water in relation to.......................... M 47 Zone of saturation........................................ W 335 Zones, deep, of ground water. 
